The General Circulation of the Atmosphere 2000 Program of Study in Geophysical Fluid Dynamics

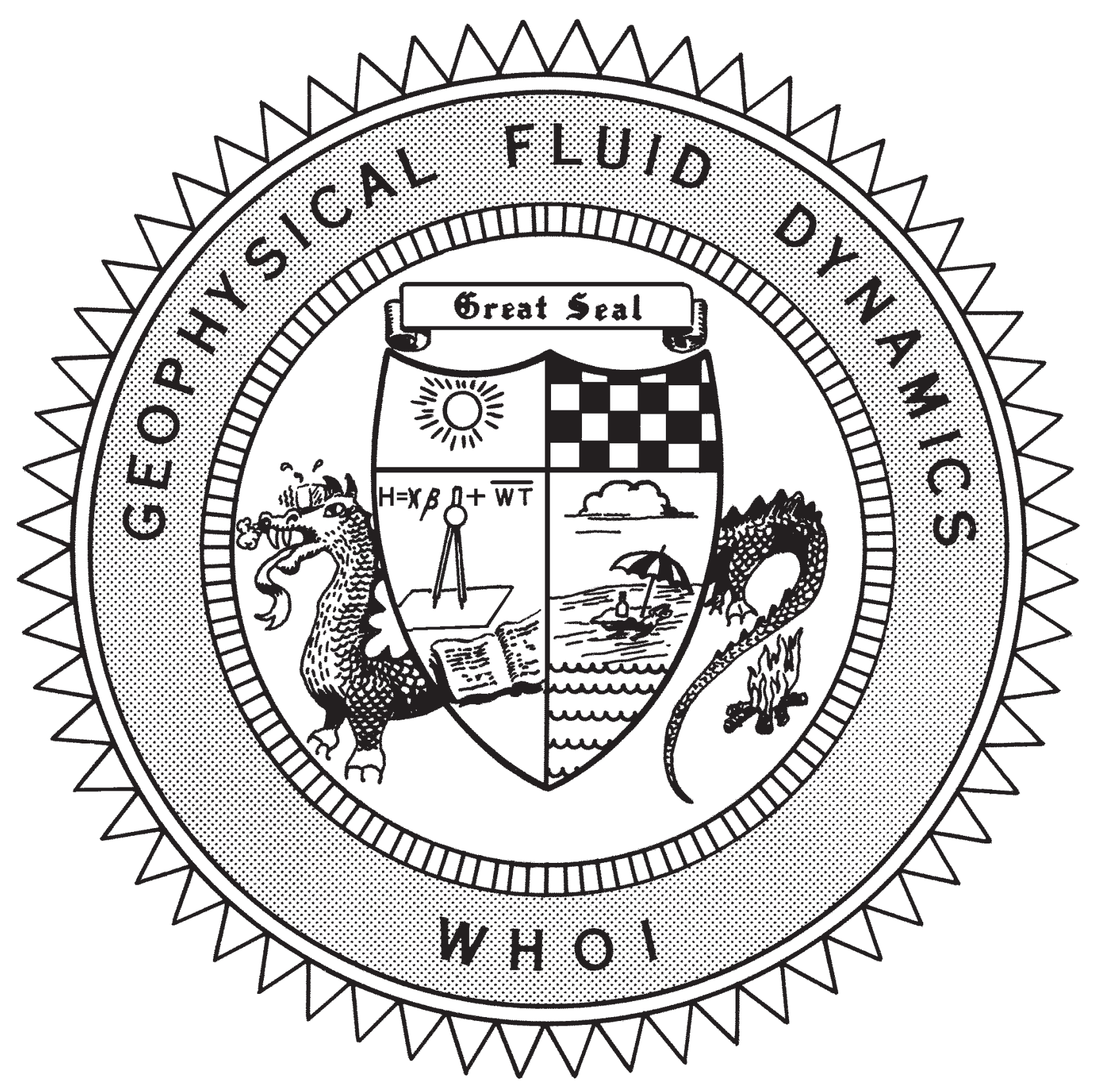

Course Lectures

Fellows Project Reports 


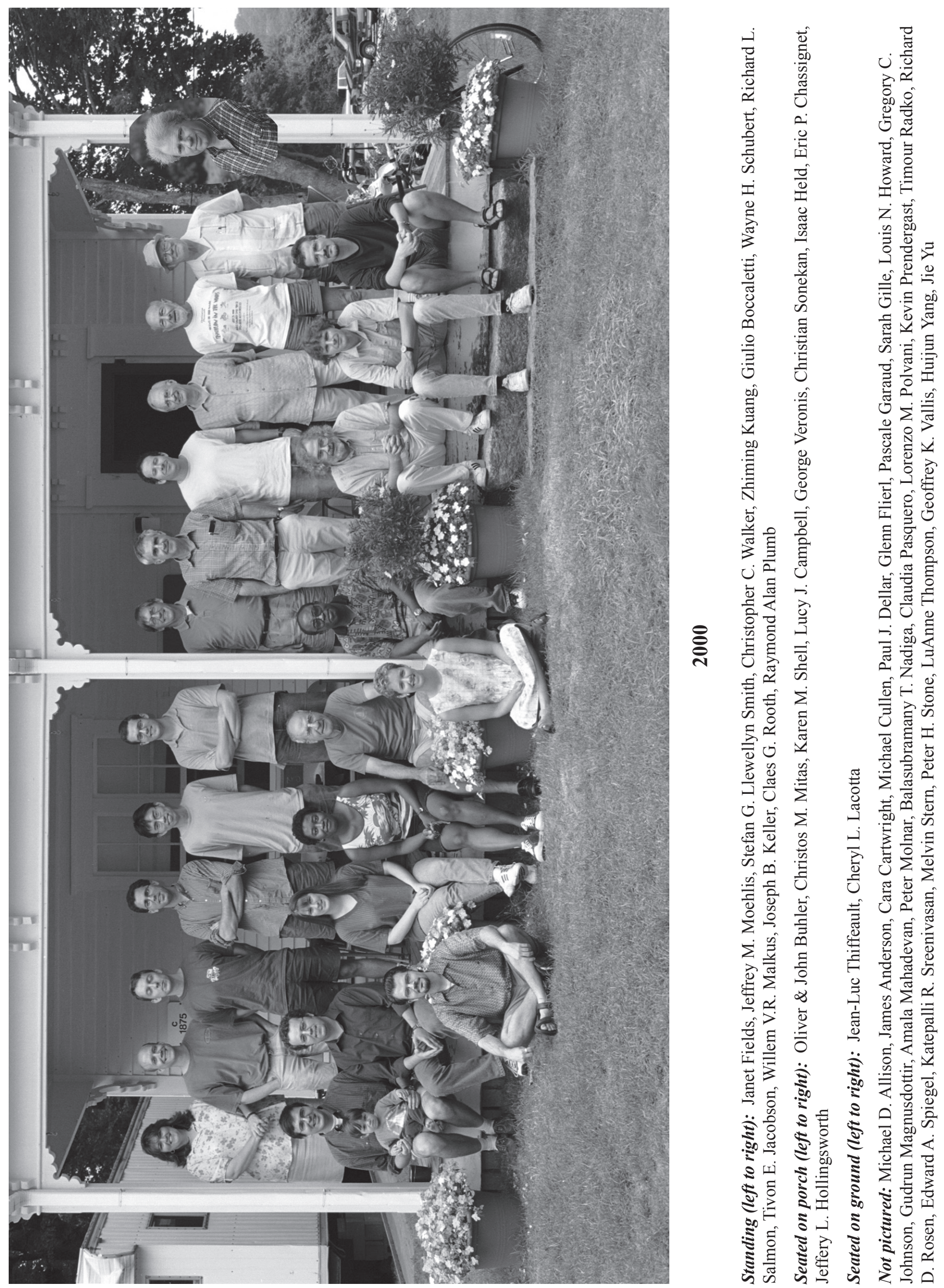




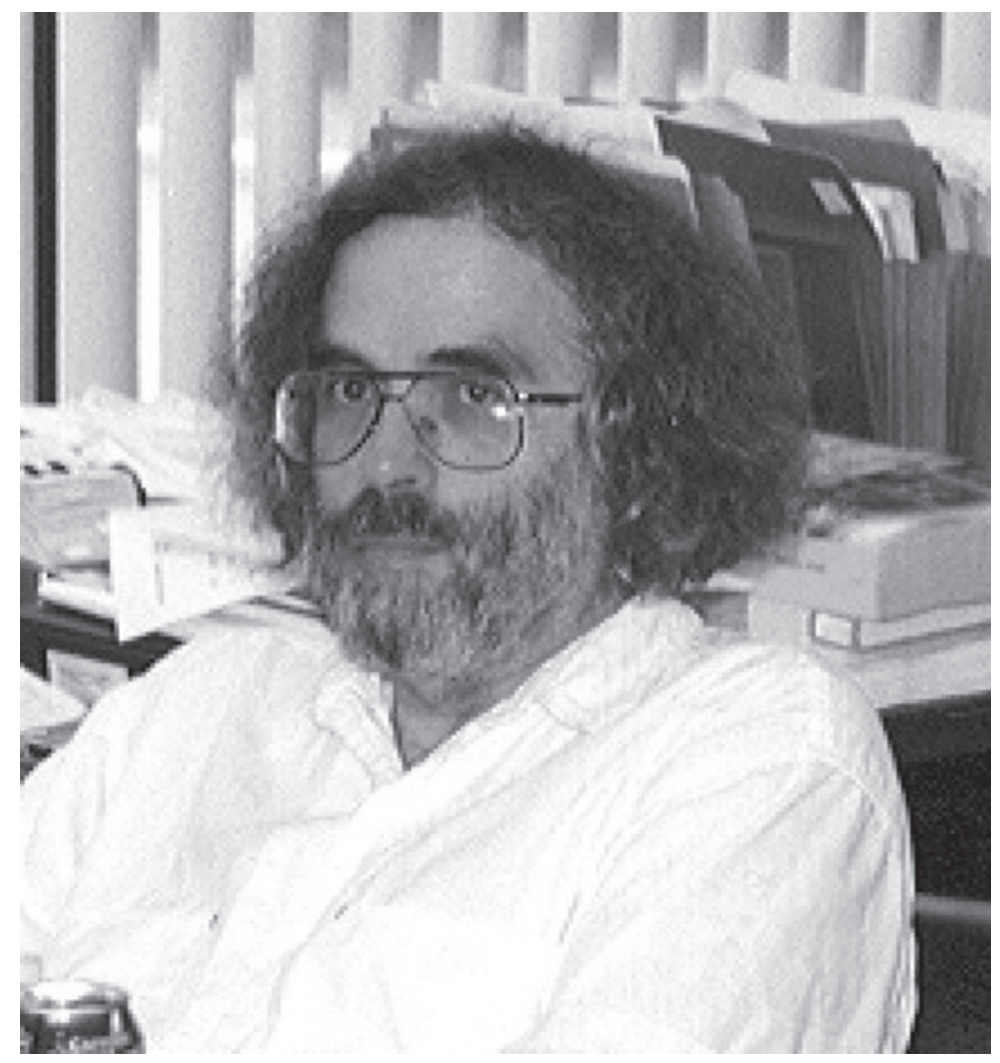

2000 GFD Principal Lecturer

Isaac Held 


\title{
The General Circulation of the Atmosphere: 2000 Program in Geophysical Fluid Dynamics
}

by

\author{
Rick Salmon, Director \\ Isaac M. Held, Principal Lecturer \\ Janet Fields, Administrator \\ Jean-Luc Thiffeault, Editor
}

March 2001

\section{Technical Report}

Funding was provided by the Office of Naval Research under Contract No. N00014-97-1-0934 and the National Science Foundation under Contract No. OCE-9810647.

Reproduction in whole or in part is permitted for any purpose of the United States Government. This report should be cited as Woods Hole Oceanog. Inst. Tech. Rept., WHOI-2001-03.

Approved for public release; distribution unlimited.

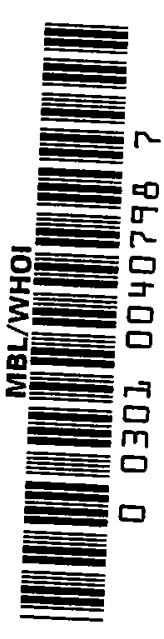

Approved for Distribution:

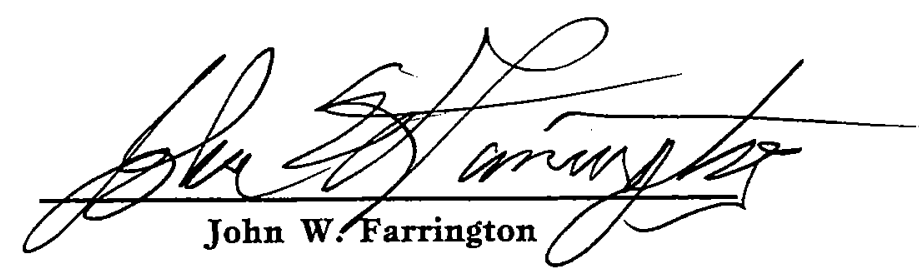

Education 


\section{PREFACE}

In its millennial session, the GFD program focused on "The General Circulation of the Atmosphere." Isaac Held led the charge with an inspiring two-week introductory course, strongly supported by the lectures of Alan Plumb, Wayne Schubert, Michael Cullen, and Oliver Bühler. As the dust settled, the important concept of pseudomomentum shone brightly in the sky above Walsh Cottage. This is a concept which has virtually transformed meteorology within the past 20 years, yet it remains mostly unfamiliar to oceanographers and others. Thus our "pseudomomentum summer" perfectly fit the GFD mission of diffusing ideas across disciplinary boundaries. As the summer wore on, the range of topics broadened in its customary way to include: the general circulation of Jupiter and Mars; lattice-Boltzmann and gas-kinetic methods for solving the equations of fluid mechanics; the scattering of sound waves by vortices; the heating of the universe; and many more topics too numerous to list. For an accurate impression of the program's activities, the reader should be sure to scan the 2000 lecture schedule.

Our 9 GFD fellows--all attending American or Canadian universities, but representing America, Italy, Barbados, Greece, Ghana and China--seemed to thrive in this atmosphere, holding up well under the pressure of the full lecture schedule and the deadline for research projects. Once again I must apologize to the many excellent applicants who were not selected to be fellows.

This year WHOI made significant improvements to Walsh Cottage, including the installation of very quiet air conditioners in the lecture room, which greatly.increased our comfort there. It is a pleasure to thank John Farrington and the Education Office staff for their indispensable help in running GFD. Very special thanks go to our administrative assistant Janet Fields for her ability to transform chaos into order, for her cheerfulness in the face of unusual requests, and for her willingness to undertake almost any mission. Eric Chassignet and Jean Luc Thiffeault generously contributed time and expertise to the maintenance of our computer network and to the preparation of this volume. Once again we acknowledge the National Science Foundation and the Office of Naval Research for their support of GFD. 


\section{TABLE OF CONTENTS}

Page

PREFACE .

i

CONTENTS $\ldots \ldots \ldots \ldots \ldots \ldots \ldots \ldots \ldots \ldots \ldots \ldots \ldots \ldots$ ii

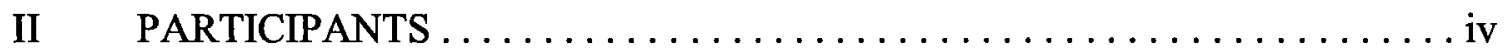

III LECTURE SCHEDULE $\ldots \ldots \ldots \ldots \ldots \ldots \ldots \ldots \ldots \ldots \ldots \ldots \ldots \ldots \ldots$

IV PRINCIPAL LECTURES - The General Circulation of the Atmosphere

Presented by Isaac M.Held, Geophysical Fluid Dynamics Laboratory, NOAA /Princeton University

Lecture One: Surface Winds and Vorticity Mixing. . . . . . . . . . . .

Lecture Two: A Linear Perspective on Eddy Momentum Fluxes. . . . . . . . . . 7

Lecture Three: A Shallow Water Model . . . . . . . . . . . . . . . . . 14

Lecture Four: A Two Layer Model. . . . . . . . . . . . . . . . . 18

Lecture Five: Continuous Stratification. .................27

Lecture Six: The Hadley Cell. . . . . . . . . . . . . . . . . . . .30

Lecture Seven: A Moist Hadley Cell. . . . . . . . . . . . . . . . . . 37

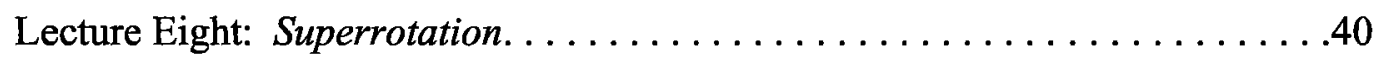

Lecture Nine: Turbulent Diffusion in Midlatitudes. . . . . . . . . . . . . 444

Lecture Ten: The Extratropical Static Stability. . . . . . . . . . . . . . . . 49

V FELLOW'S LECTURES

Fellow's Report One:

Monsoons in a Moist Axially Symmetric Model of the Atmosphere

Giulio Boccaletti, Princeton University. . . . . . . . . . . . . . . . 55

Fellow's Report Two:

A Quasi-Biennial Oscillation Generated by Gravity Wave Breaking

Lucy Campbell, McGill University, CANADA. . . . . . . . . . . . . . . .70 
V FELLOW'S LECTURES, continued

Fellow's Report Three:

Vorticity on a Barred Beach

Tivon Jacobson, New York University.

Fellow's Report Four:

A Truncated Model of Finite-Amplitude Baroclinic Waves in a Channel

Zhiming Kuang, California Institute of Technology. . . . . . . . . . . . . . . .96

Fellow's Report Five:

Can a Simple Two-Layer Model Capture the Structure of Easterly Waves?

Cheryl Lacotta, University of Arizona. . . . . . . . . . . . . . . . . 108

Fellow's Report Six:

Eddy PV Fluxes in a One-Dimensional Model of Quasi-Geostrophic

Turbulence

Christos Mitas, University of Illinois. . . . . . . . . . . . . . . . . . . 120

Fellow's Report Seven:

Superrotation in an Axisymmetric Shallow Water Model of the Upper

Troposphere

Karen Shell, Scripps Institution of Oceanography.

Fellow's Report Eight:

What Makes Oceanic Gravity Currents Flow Downhill

Christopher Walker, University of California, Irvine.

Fellow's Report Nine:

Experimental Investigation of a Theory for Oceanic Convection

Christian Sonekan, University of Charleston. 


\section{GFD FELLOWS, STAFF AND VISITORS}

\section{Fellows}

Giulio Boccaletti

Lucy J. Campbell

Tivon E. Jacobson

Zhiming Kuang

Cheryl L. Lacotta

Christos M. Mitas

Karen M. Shell

Christian Sonekan

Christopher C. Walker
Princeton University

McGill University, CANADA

New York University

California Institute of Technology

University of Arizona

University of Illinois

Scripps Institution of Oceanography

University of Charleston

University of California, Irvine

\section{Staff and Visitors}

Michael D. Allison

James Anderson

Oliver Buhler

Cara Cartwright

Eric P. Chassignet

Michael Cullen

Paul J. Dellar

Glenn Flierl

Pascale Garaud

Sarah Gille

Isaac Held

Jeffery L. Hollingsworth

Louis N. Howard

Gregory C. Johnson

Joseph B. Keller

Stefan G. Llewellyn Smith

Gudrun Magnusdottir

Amala Mahadevan

Willem V.R. Malkus

Jeffrey M. Moehlis

Peter Molnar

Balasubramany T. Nadiga

Claudia Pasquero

Raymond Alan Plumb

Lorenzo M. Polvani

Kevin Prendergast

Timour Radko

Claes G. Rooth

Richard D. Rosen

Richard L. Salmon

Wayne H. Schubert

Edward A. Spiegel
Goddard Institute for Space Studies

Stevens Institute of Technology

University of St. Andrews, UNITED KINGDOM

Princeton University

University of Miami

European Center For Medium Range Weather Forecasts

St. John's College, UNITED KINGDOM

Massachusetts Institute of Technology

University of Cambridge, UNITED KINGDOM

University of California, Irvine

Geophysical Fluid Dynamics Lab./NOAA

NASA Ames Research Center/SJSUF

Florida State University/Massachusetts Inst. of Technology

National Oceanic and Atmospheric Administration

Stanford University

University of California, San Diego

University of California, Irvine

Atmospheric and Environmental Research, Inc.

Massachusetts Institute of Technology

Princeton University

Massachusetts Institute of Technology

Department of Energy, Los Alamos National Lab

Istituto di Cosmogeofisica/CNR, ITALY

Massachusetts Institute of Technology

Columbia University

Columbia University

Florida State University

University of Miami

Atmospheric and Environmental Research, Inc.

University of California, San Diego

Colorado State University

Columbia University 
Katepalli R. Sreenivasan

Melvin Stern

Peter H. Stone

Jean-Luc Thiffeault

LuAnne Thompson

Geoffrey K. Vallis

George Veronis

Huijun Yang

Jie $\mathrm{Yu}$
Yale University

Florida State University

Massachusetts Institute of Technology

Columbia University

University of Washington

Princeton University

Yale University

University of South Florida

The Florida Atlantic University 


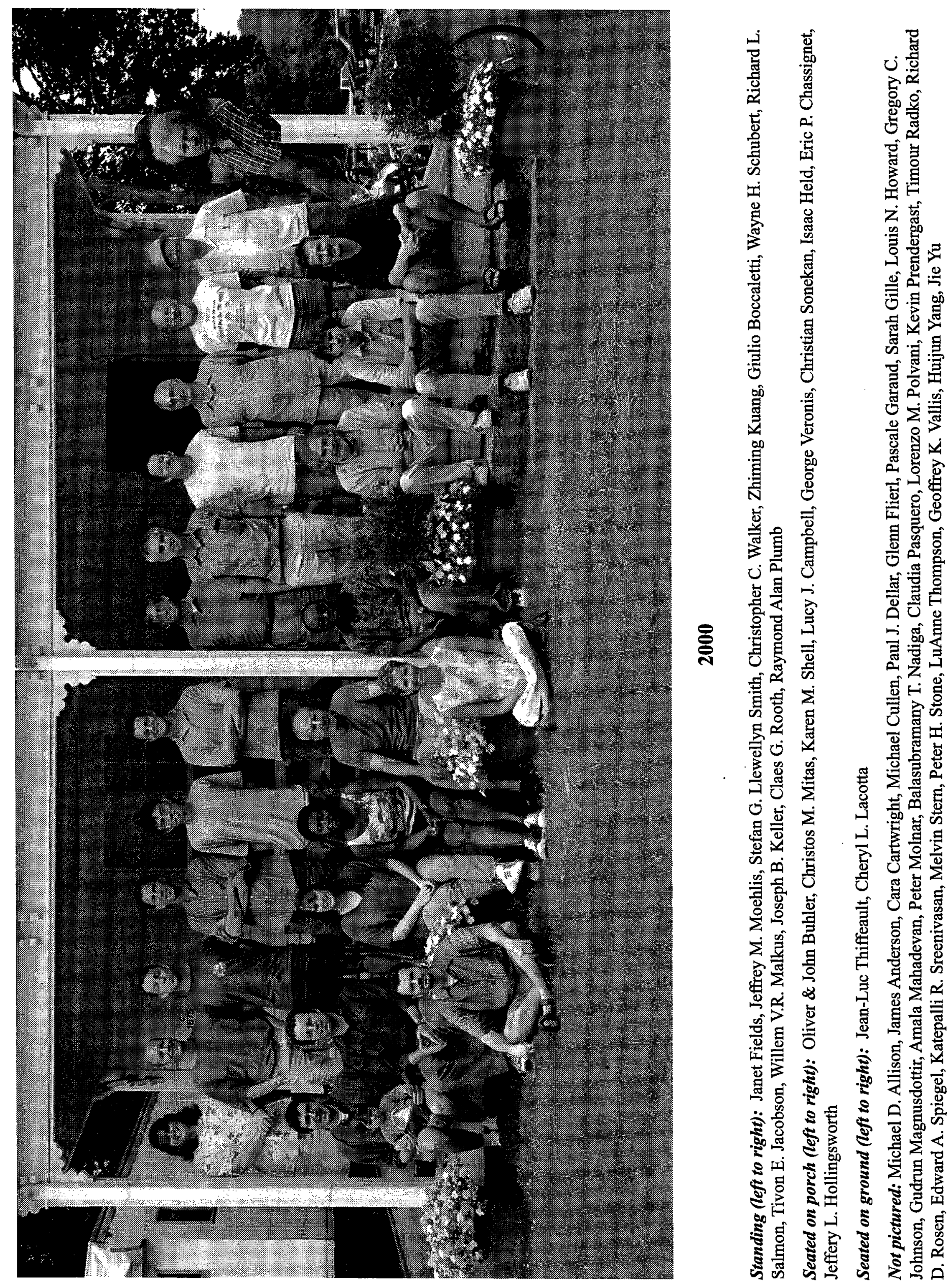




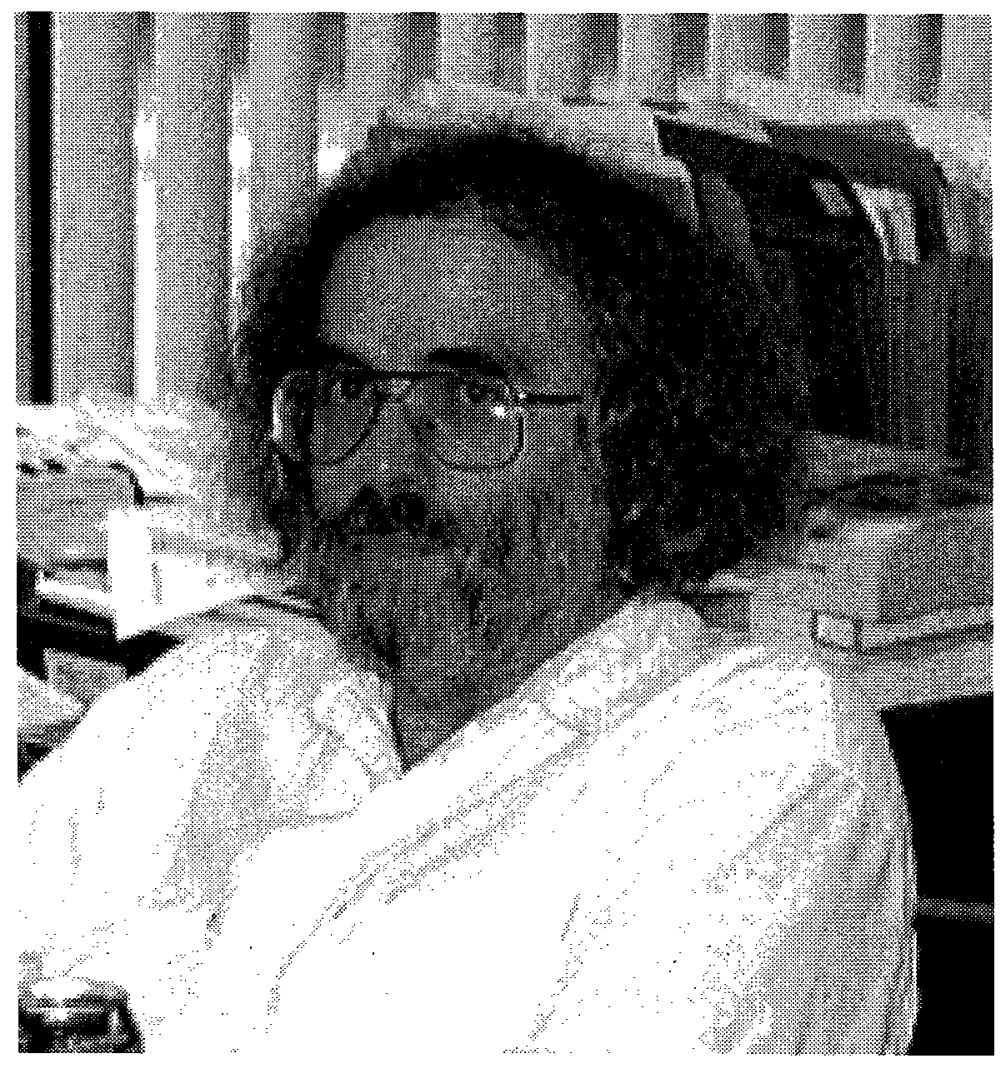

2000 GFD Principal Lecturer

Isaac Held 


\section{GFD 2000 Lecture Schedule}

Week of June $19-23,2000$

(All talks held in Walsh cottage inless othenwise noted.)

Monday, June 19

10:00 am

Tuesday, June 20

10:00 am

Wednesday, June 21

10:00 am

Thursday, June 22

10:00 am
Isaac M.Held, Geophysical Fluid Dynamics Laboratory, NOAA /

Princeton University

Surface Winds and Vorticity Mixing

Isaac M.Held

A Linear Perspective on Eddy Momentum Fluxes

Isaac M.Held

A Shallow Water Model

Isaac M.Held

A Two Layer Model

Friday, June 23

10:00 am
Isaac M.Held

Continuous Stratification

\section{Week of June $26-30,2000$}

Monday, June 26

10:00 am

Tuesday, June 27

10:00 am

Isaac M.Held

A Moist Hadley Cell

Wednesday, June 28

10:00 am

$3: 30 \mathrm{pm}$
Isaac M.Held

The Hadley Cell
Isaac M.Held

Superrotation

Wayne Schubert, Colorado State University

Interpretting the ITCZ and Hadley Circulation in Terms of Potential Vorticity Dynamics 
Thursday, June 29

10:00 am

Isaac M.Held

Turbulent Diffusion in Midlatitudes

2:00 pm

Alan Plumb, Massachussettes Institute of Technology

Monsoons: Localized Hadley Circulations

Friday, June 30

10:00 am

Isaac M.Held

The Extratropical Static Stability

Week of July $3-7,2000$

Monday, July 3

10:00 am

Mike Cullen, European Center for Medium Range Weather Forecasts

Understanding Mesoscale Dynamics Using

Semi-Geostrophic Theory

Tuesday, July 4

No Lecture

Wednesday, July 5

10:00 am

Mike Cullen, European Center for Medium Range Weather Forecasts

Understanding Large-Scale Atmospheric Circulations Using

Semi-Geostrophic Theory

Thursday, July 6

10:00 am

Oliver Buhler, University of St. Andrews, UK

Wave-mean Interactions Involving Gravity Waves

2:00 pm Paul Dellar, University of Cambridge, UK

Lattice Boltzmann Methods for GFD

Friday, July 7

10:00 am

Oliver Buhler, University of St. Andrews, UK

Gravity Wave Parametrizations and Conservation Laws

Week of July $10-14,2000$

Monday, July 10

10:00 am

Geoff Vallis, GFDL/Princeton University

Equilibration of Eddies in the Ocean and Atmosphere

Tuesday, July 11

10:00 am

Jeff Moehlis, Princeton University

Bursts in Binary Fluid Convection 
Wednesday, July 12

10:00 am

Thursday, July 13

10:00 am

Friday, July 14

10:00 am
Eric Chassignet, University of Miami

On the Importance of Surface Forcing in Numerical Simulations of the North Atlantic Ocean

Paul Dellar, University of Cambridge, UK

Selective Decay and Geostrophic Adjustment

Joe Keller, Stanford University

Walking On Water

\section{Week of July 17 - 21, 2000}

Monday, July 17

10:00 am

Wayne Schubert, Colorado State University

Potential Vorticity in a Moist Atmosphere

2:00 pm

Brian Arbic, MIT/WHOI Joint Program

What Controls the Vertical Structure and Lengthscales of Mid-Ocean Eddies?

Tuesday, July 18

10:00 am

Gudrun Magnusdottir, University of California, Irvine

The Response of the Atmospheric Heat Transport to Different

Configurations of Implied Oceanic Heat Transport

Wednesday, July 19

10:00 am

Michael Allison, NASA Goddard Institute for Space Studies

PV Perspectives on Planetary Circulations

2:00 pm Huijun Yang, University of South Florida

A Simple Theory for Ocean Climate Variability

Thursday, July 20

10:00 am

Alan Plumb, Massachusetts Institute of Technology

Three-Dimensional Eddy Parameterization

Friday, July 21

10:00 am

Michael Allison, NASA Goddard Institute for Space Studies A Dynamical Model for Jupiter's Thermocline

Week of July $24-28,2000$

Monday, July 24

10:00 am

Alan Plumb, Massachusetts Institute of Technology

Circulation in the Stratosphere 
Tuesday, July 25

10:00 am

Wednesday, July 26

10:00 am

8:00 pm

Thursday, July 27

10:00 am

Friday, July 30

10:00 am
Kevin Prendergast, Columbia University

Barred Spirals and BGK Hydrocodes

Isaac Held, Geophysical Fluid Dynamics Laboratory,

NOAA / Princeton University

Surface Quasigeostrophic Turbulence

*Special Evening Lecture*

Ed Spiegel, Columbia University

Heating the Universe

Rick Rosen, Atmospheric and Environmental Research, Inc.

Angular Momentum of the 20th and 21st Centuries

Jean-Luc Thiffeault, Columbia University

The Wondrous Exponents of Dr. Lyapunov

\section{Week of July 31 - Augast 4,2000}

Monday, July 31

10:00 am

Tuesday, August 1

10:00 am

Wednesday, August 2

10:00 am

2:00 pm

Thursday, August 3

10:00 am

Friday, August 4

10:00 am
Isaac Held, Geophysical Fluid Dynamics Laboratory,

NOAA / Princeton University

Some Aspects of Turbulent Moist Convection

Balu Nadiga, Los Alamos Laboratory

A New Dynamical Mechanism for Variabilty in the Double

Gyre Circualtion

Stefan Llewellyn-Smith, University of California, San Diego

Wave Scattering by Vortices

Balu Nadiga, Los Alamos Laboratory

Four-gyre Circulation With Double-gyre Wind Forcing and Eddy

Parameterization: Barotropic Case

Wayne Schubert, Colorado State University

Hurricane Dynamics

Lou Howard, Massachusetts Institute of Technology/FSU

Weak Water Waves Washing Widely Over a Wavy Wall 


\section{Week of August 7 - 11, 2000}

Monday, August 7

10:00 am

2:00 pm

Tuesday, August 8

10:00 am

Wednesday, August 9

10:00 am

Thursday, August 10

10:00 am
Amala Mahadevan, Atmospheric and Environmental Research, Inc.

The Impact of Fronts on Upper Ocean Biogeochemistry

Willem Malkus, Massachusetts Institute of Technology

Disorder to Order

Jeff Hollingsworth, NASA, Ames Research Center/SJSUF

General Circulation and Modeling of Mars' Atmosphere

Jeff Hollingsworth, NASA, Ames Research Center/SJSUF

Cyclogenesis and Frontal Waves During Northern Early Spring

and Late Summer on Mars

John Marshall, Massachusetts Institute of Technology

Circumpolar Currents

Friday, August 11

2:00 pm
Oliver Buhler, University of St. Andrews, UK

Statistical Mechanics of Beached Vortices

\section{Week of August 14-18, 2000}

Monday, August 14

10:00 am

Tuesday, August 15

10:00 am

Wednesday, August 16

10:00 am

Thursday, August 17

10:00 am

Friday, August 18

10:00 am
Claes Rooth, University of Miami

Ocean-Atmosphere Energy Flux Partitioning

Luanne Thompson, University of Washington

The Influence of the Seasonal Cycle on Rossby Waves

Lenny Smith, Oxford University

Predicting Chaos: How is the Atmosphere Different From an

Electric Circuit?

Jim Anderson, Stevens Institute of Technology

What is a Macroscopic Variable?

Jie Yu, Florida Atlantic University

Waves and Nearshore Currents 


\section{Week of August 21 - 25, 2000-Fellows' Lectures}

Monday, August 21

Tuesday, August 22

10:00 am

11:00 am

2:00 pm

$3: 00$ pm

Wednesday, August 23

10:00 am

11:00 am

2:00 pm

3:00 pm

4:00 pm
No Lecture

Tivon Jacobson, New York University

Vorticity on a Barred Beach

Christopher Walker, University of California, Irvine

What Makes Oceanic Gravity Currents Flow Downhill

Christos Mitas, University of Illinois

Eddy PV Fluxes in a One-Dimensional Model of QuasiGeostrophic Turbulence

Cheryl Lacotta, University of Arizona

Can a Simple Two-Layer Model Capture the Structure of Easterly Waves?

Karen Shell, Scripps Institution of Oceanography Superrotation in an Axisymmetric Shallow Water Model of the Upper Troposphere

Giulio Boccaletti, Princeton University Monsoons in a Moist Axially Symmetric Model of the Atmosphere

Zhiming Kuang, California Institute of Technology A Truncated Model of Finite-Amplitude Baroclinic Waves in a Channel

Christian Sonekan, University of Charleston Experimental Investigation of a Theory for Oceanic Convection

Lucy Campbell, McGill University, CANADA

A Quasi-Biennial Oscillation Generated by Gravity Wave Breaking 


\title{
The General Circulation of the Atmosphere
}

\author{
Isaac M. Held and GFD/2000 Fellows
}

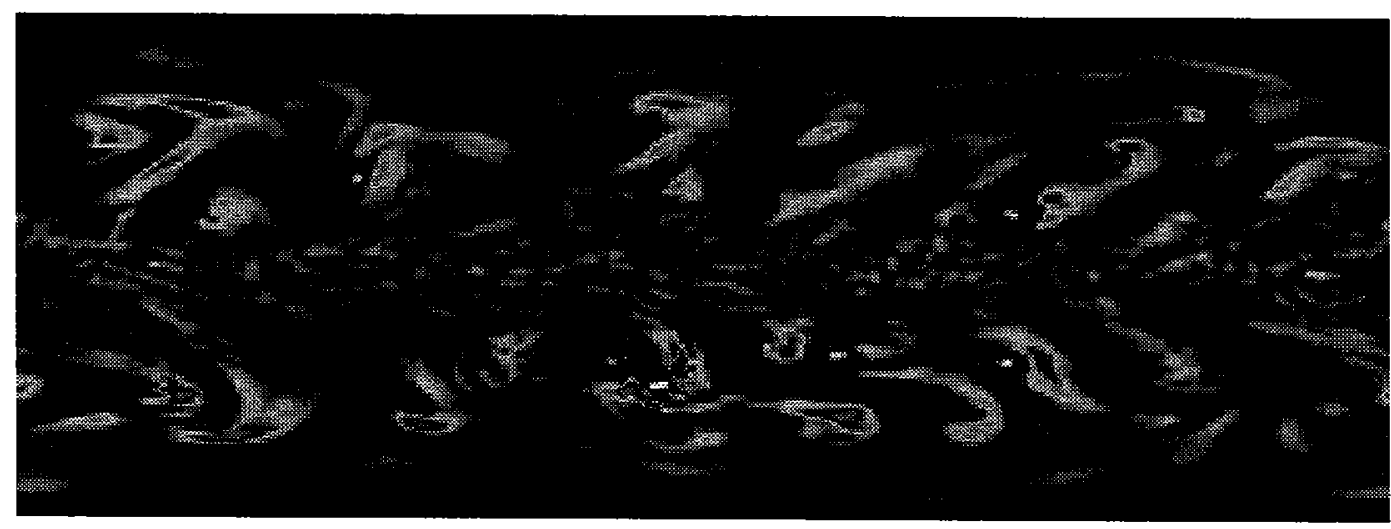

Figure 1: Mid-tropospheric vertical motion in an idealized dry atmospheric model with a zonally symmetric climate, forced as described in [7]. The entire sphere is shown. Note the wave-like structures in midlatitudes (with a NE/SW tilt in the Northern subtropics and the opposite tilt in the Southern subtropics) and the more turbulent, convective, character of the tropics. The model is spectral with T106 resolution. Green $\rightarrow$ upward; red $\rightarrow$ strongly upward; blue $\rightarrow$ downward; yellow $\rightarrow$ strongly downward.

\section{Surface winds and vorticity mixing}

Our goal in these lectures will be to develop a qualitative understanding of the general circulation of the atmosphere. As computer power increases, the problem of the general circulation is more and more often tackled with complex numerical simulations. Yet attempts at comprehensive simulations in isolation rarely produce satisfactory understanding. The challenge for theorists in the future will be to combine idealized models and complex, more realistic simulations in such a way as to produce a deeper understanding of the atmospheric circulation.

Studies of the general circulation focus on large scale structures of the atmospheric climate. These include the seasonally varying mean state and the statistics of eddies, on various space and time scales, that influence this mean state. Surveys of existing meteorological data define most of these large-scale structures rather well. The latitude-height distribution of the zonally averaged zonal winds is an important example (Figure 2) on which we will focus much of our attention. 


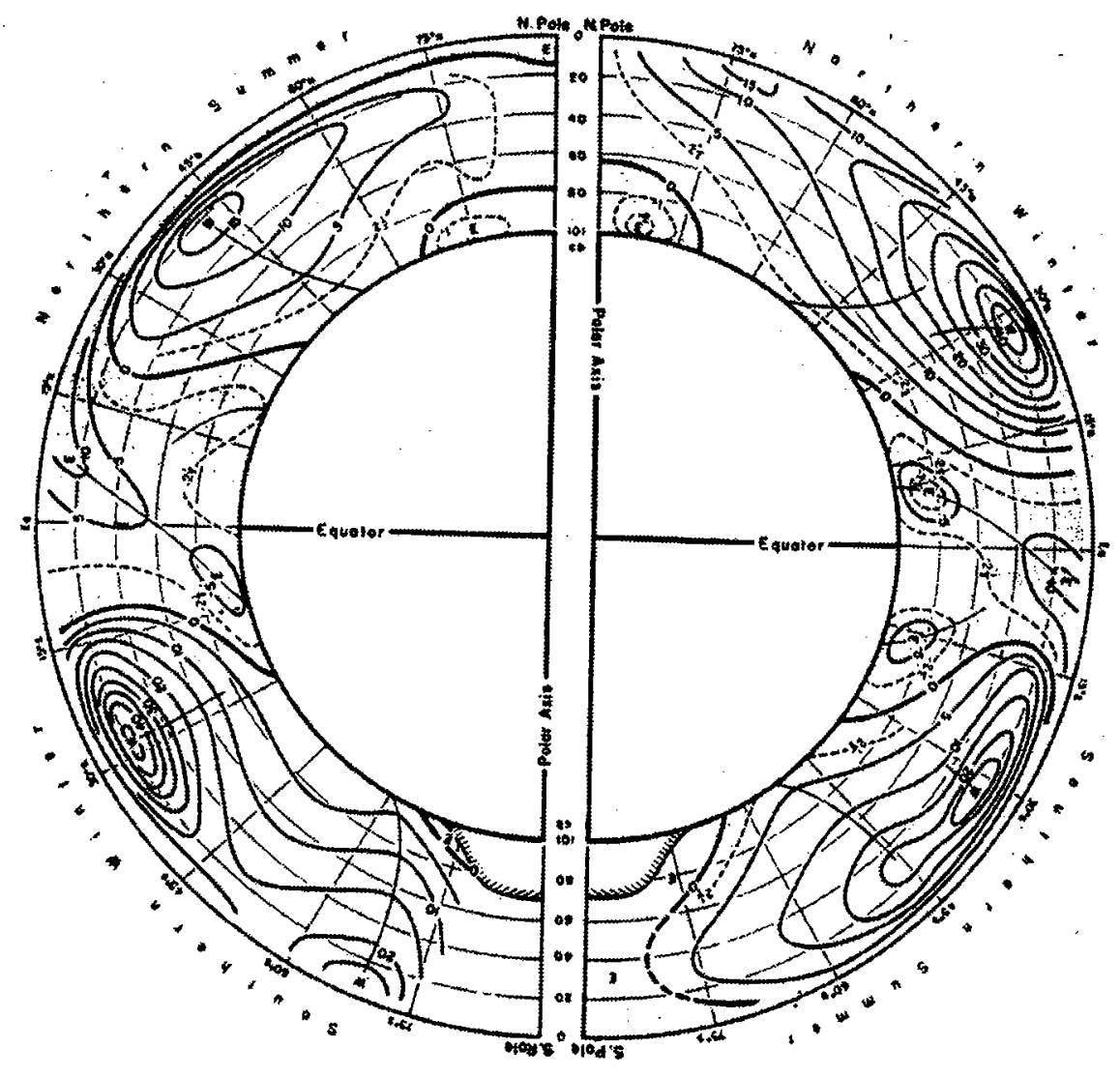

Figure 2: Zonally averaged zonal wind as a function of pressure and latitude for the summer and winter seasons in both Northern and Southern hemispheres, from [11]. The contour interval is $5 \mathrm{~m} / \mathrm{s}$. The vertical axis is marked every $200 \mathrm{mb}$. 
If the Earth's surface were zonally symmetric (independent of longitude), the atmospheric climate would be zonally symmetric as well. While there are substantial asymmetries in the surface, of course, due to mountains and the land-sea distribution (and the ocean circulation), the basic structure of the zonally averaged atmospheric climate does not depend strongly on these details. The similarity between the zonal mean flow in the Northern and Southern Hemispheres, despite the very different lower boundary structures, is good evidence of this. (The tendency for the upper tropospheric flow in the Southern winter to split into a subtropical and a subpolar jet in the zonal mean is the most distinctive interhemispheric difference in Figure 2.) There is a lot of interesting theory that helps us understand the deviations from zonal symmetry of the climate, but this topic will not be addressed here. Rather, we will focus on a hypothetical Earth with a symmetric lower boundary and a symmetric climate.

There are many starting points that one could choose in a discussion of the atmospheric climate. I have chosen the transport of angular momentum. (The term "angular momentum" in these lectures always refers to the component of the angular momentum vector in the direction of the Earth's rotation.) At first, we will focus on the horizontal redistribution of angular momentum by the extratropical circulation, which is intimately tied to the maintenance of the zonal mean surface wind distribution. Then we will move on to the vertical redistribution of angular momentum, which controls the vertical shear of the zonal wind in the extratropics, or, equivalently, the north-south temperature gradient. The angular momentum budget also provides the key ingredient in understanding the fundamental dynamical distinction between the tropical and extratropical atmospheres. We will touch on the interaction of moist convection and large-scale dynamics only in the discussion of the Hadley circulation.

The basic features of the zonal winds are very familiar. At the surface, we see easterlies in low latitudes, westerlies in midlatitudes, and weak easterlies again near the poles. The surface pressure distribution is in approximate geostrophic balance with these zonal winds, with subtropical highs and subpolar lows. The zonal force balance will be of greater interest to us than the meridional force balance, however. The zonal component of the pressure gradient averages to zero when integrated around a latitude circle. Therefore, the dominant terms in the zonally averaged zonal component of the force balance near the surface are the Coriolis force resulting from north-south motion and the frictional torques that retard the zonal winds. Therefore, the existence of an eastward frictional torque in the tropical region of surface easterlies requires, in a steady state, a zonally averaged equatorward flow, in order for the Coriolis force on this flow to balance the frictional torques. The return flow occurs near the tropopause. This tropical circulation is referred to as the Hadley cell. Over the midlatitude westerlies, the same argument requires the opposite sense of meridional overturning, referred to as the Ferrel cell. A weak polar cell over the polar surface easterlies completes the three-cell picture (see figure 3). The mass transports in the surface branches of these atmospheric meridional circulations are simply the boundary layer Ekman mass transports that balance the surface stresses.

In addition to the surface winds, we see in Figure 2 the familiar increase in the westerlies with height, consistent with the thermal wind equation and the north-south temperature gradient. If we have an understanding of the surface wind field and of the north-south temperature gradients, we have an understanding of the upper tropospheric flow as well. 


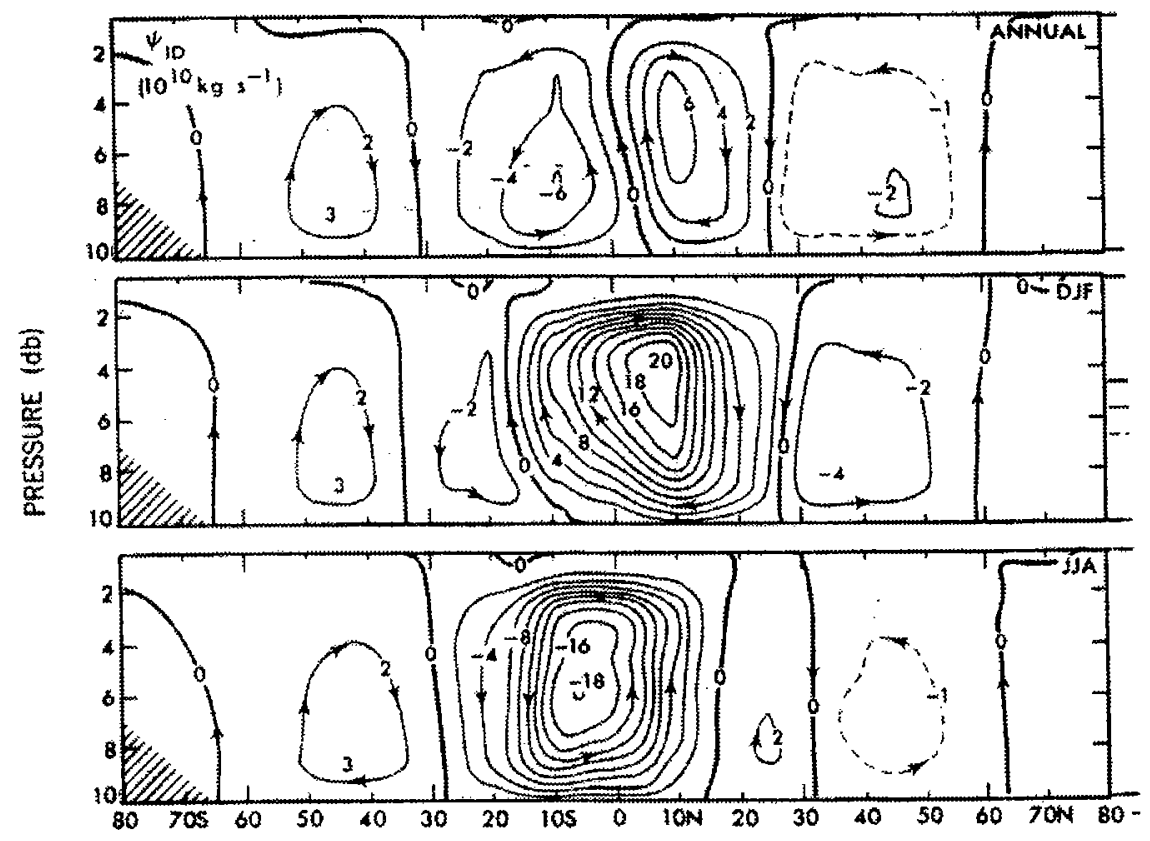

Figure 3: The traditional overturning streamfunction, shown for the annual mean and the summer and winter seasons. The contour interval is 10 Sverdrups; from [14]

A key feature in the mean circulation is the upper tropospheric subtropical jet, which can usefully be thought of as marking the boundary between the tropics and extratropics. Its latitude, to a good approximation, is also the latitude of the boundary between surface easterlies and westerlies.

The angular momentum per unit mass, $M$, is defined as

$$
M=(u+\Omega a \cos \theta) a \cos \theta
$$

where $\Omega$ is the angular velocity of the Earth, $u$ the zonal flow of the atmosphere with respect to this solid body rotation, $a$ the radius of the Earth, and $\theta$ is latitude. The first term is the contribution from the solid body rotation and the second term the contribution due to departures from solid body rotation. (We have made a thin shell approximation by assuming that the distance to the axis of rotation is simply $a \cos \theta$, independent of the height of the parcel above the surface.) The total angular momentum integrated over the atmosphere does not vary in time when averaged over a long enough time period

$$
\frac{\partial}{\partial t} \int \rho M d V \approx 0
$$

Due to surface frictional stresses, and form drag due to correlations between the surface pressure and the east-west slope of the surface, both of which tend to oppose the near-surface winds, angular momentum is transferred from the atmosphere to the Earth in midlatitudes and from the Earth to the atmosphere in the tropics and the polar regions. To obtain a 
steady state, angular momentum must be transported from the tropics and the polar regions to midlatitudes. The dominant path by far is that from the tropics to midlatitudes.

The poleward flux of angular momentum can be broken into contributions from the zonal mean flow (denoted by an overbar) and deviations from this mean (denoted by a prime)

$$
\overline{v M}=\bar{v} \bar{M}+\overline{v^{\prime} M^{\prime}}
$$

As first hypothesized by Jefferies in the 1920's and confirmed by observations when upper air data became available after WWII, this angular momentum transfer is mainly accomplished by large-scale eddies. Most of this eddy momentum flux occurs in the upper troposphere. The mean meridional circulation is too weak to make a significant contribution, except in the deep tropics. Understanding the distribution of surface winds and surface stresses is equivalent (outside of the deep tropics) to understanding the convergence of the eddy fluxes of angular momentum.

As a simple starting point, assume the upper troposphere can be modeled as a homogeneous $\rho=$ const, nearly inviscid, nondivergent flow $\nabla \cdot \mathbf{u}=0$ in a infinitesimally thin spherical shell. We first need to review two basic facts about vorticity: Stokes' Theorem (a kinematic result from vector calculus) and Kelvin's Circulation Theorem (a dynamic result for this homogeneous model).

By Stokes' Theorem, the circulation, the line integral of the velocity, around any closed loop is equal to the normal component of the vorticity, the curl of the velocity, integrated over any surface bounded by the loop:

$$
\oint \mathbf{u} \cdot \mathrm{dl}=\iint \omega \cdot \hat{\mathbf{n}} d A
$$

where $\omega$ is the vorticity. If the loop is a latitude circle, we see that the circulation is just the zonally-averaged flow $\bar{u}$ times the length of a latitude circle. By choosing the bounding surface to be the surface of the sphere itself, we also see that the zonally averaged flow at some latitude is also the radial component of vorticity integrated over the polar cap bounded by this latitude circle, divided by the length of the latitude circle.

Kelvin's Circulation Theorem states, for our homogeneous, incompressible and inviscid fluid, that the circulation around a material loop (one moving with the flow), does not change in time. By Stokes' Theorem, it follows that the time derivative of the surface integral of the normal component of the vorticity, over any surface bounded by this material loop, is also equal to zero. If we specialize to the case of an infinitesimal loop, we have

$$
\frac{D}{D t}(\omega \cdot \mathbf{n} d A)=0 \text {. }
$$

where $\mathbf{n}$ is the normal to the loop and $d A$ is its area. For the nondivergent flow on a sphere considered here, the area of a material loop cannot change. Thus, the radial component of vorticity is conserved following parcels on a spherical surface.

In solid body rotation with angular velocity $\boldsymbol{\Omega}$, the radial component of the vorticity is $2 \Omega \sin (\theta)$. It is fundamental to large-scale atmospheric and oceanic flows that this is a monotonically increasing function of latitude, from the south pole to the north pole.

Suppose we stir the system with a 'spoon' at midlatitudes such that no net zonal momentum is added to the upper troposphere. (The latter restriction is meant to focus attention 
on horizontal redistribution of angular momentum; as we will see, the appropriate model of this stirring will, in fact, include a net tranfer of angular momentum from the upper to the lower troposphere.) Consider a latitude circle outside of the stirring region. If the disturbance reaches this location, the line of particles initially on the latitude circle will distort, conserving the total area encompassed by this line. When the particles move off the latitude circle, they conserve the radial component of their vorticity. Assume that the initial condition is close enough to solid body rotation that the vorticity distribution is monotonic. Then when the ring of particles deforms, high vorticity particles move south across the latitude circle and low vorticity particles move north. The net effect is an equatorward flux of vorticity at this latitude.

Thus, integrated over the polar cap bounded by this latitude circle, the radial component of vorticity will decrease. Therefore, by Stokes' Theorem, we know that $\bar{u}$ will also decrease (i.e. accelerate westward). Since we assumed no net angular momentum is imparted by the spoon to the layer as a whole, and since there is westward acceleration everywhere except in the region directly stirred by the spoon, the region stirred by the spoon must be accelerating eastward. Angular momentum converges into the 'stirred' region.

How can this convergence be maintained? Suppose that the stirring is episodic. After a burst of eddy activity, the flow might reversibly return to its initial condition, in which case the vorticity transport and associated convergence of angular momentum would be reversed during the decay of the disturbance. However, if the stirring generates irreversible mixing (i.e. pieces of vorticity break off and mix into the surrounding air), we cannot return to the initial state. Thus, we need to include some irreversibility in our model, we need to mix vorticity, to create sustained convergence of angular momentum.

Additionally, as this irreversible mixing continues, the gradients of vorticity will decrease and eventually disappear where this mixing is strongest, after which there will be no more vorticity transport and momentum flux convergence in these regions. If we are to take this barotropic model seriously as a model of a statistically steady state, we would need some sort of mechanism to restore the vorticity gradient. At this point, this model becomes a bit artificial - in the more realistic baroclinic case, as we will see, it is the fluxes of potential vorticity that play the role that vorticity fluxes play here, and (unlike vorticity) potential vorticity gradients can be restored by radiative heating.

This barotropic model is meant as a model of the upper troposphere, where the eddy momentum fluxes are concentrated. In midlatitudes there are no other mechanisms besides these upper tropospheric eddy fluxes that transfer significant amounts of angular momentum meridionally. The converging angular momentum in the midlatitude (stirred) region must be removed somehow. The only mechanism available is removal at the surface. How the momentum gets to the surface from the upper troposphere will be a dominant theme later in these lectures, For the time being we need only realize that it must get there somehow so as to be removed by surface torques.

Therefore, we should think of the midlatitude westerlies as existing so as to remove the angular momentum being sucked into these latitudes in the upper troposphere. This sucking is created by the meridional spreading of eddies away from their region of excitation in midlatitudes. Westerlies appear under the stirred region.

These upper tropospheric eddy momentum fluxes force the surface stress distribution and the associated low level wind field. They do not explain the upper tropospheric flow, 
which is more strongly controlled by the vertical shears. It is a common mistake to talk of how horizontal eddy momentum fluxes "maintain" the upper level winds. One should think of the upper level flow as the sum of the surface winds and the vertical wind shear. Only the former is closely tied to the eddy momentum fluxes.

We gain some intuition about momentum fluxes, not by thinking about the momentum equation directly, but by thinking about vorticity fluxes and changes in circulation. It is because vorticity is conserved following fluid parcels, in this barotropic model, that we can gain some intuitive feel for the structure of the eddy vorticity fluxes.

This model does not explain why the stirring occurs preferentially in midlatitudes on our Earth. In fact, as the rotation rate is increased in Earth-like climate models, one generally finds that the three-cell circulation is replaced by a five-cell pattern, with two regions of surface westerlies per hemisphere [22]. In idealized quasi-geostrophic models, it is easy to generate a dozen or more bands of surface westerlies [12]. The "stirring" evidently organizes itself in these models into multiple bands when the eddy scale is much smaller than the size of the domain. On Earth, the eddy scale is large enough that we have only one such band.

\section{A linear perspective on eddy momentum fluxes}

The discussion of meridional angular momentum redistribution in Lecture 1 did not make any reference to Rossby waves, or to any linearized dynamics for that matter. But by considering linear dynamics on a stable zonal flow we can gain additional perspective on the central result that a rapidly rotating flow, when stirred in a localized region, will converge angular momentum into this region.

The disturbances that carry angular momentum meridionally in the upper troposphere are, in fact, rather wavelike. We can decompose the covariance between $u$ and $v$ into contributions from different space and time scales by Fourier decomposing both fields in the zonal direction and in time. We can write the resulting co-spectrum as a function of zonal wavenumber $k$ and frequency $\omega$, or, equivalently, as a function of wavenumber and phase speed, $c \equiv \omega / k$ :

$$
\overline{u^{\prime} v^{\prime}}=\sum_{k} \int d c M(k, c)
$$

Shown in Figure 4 is $M(k, c)$ integrated over all wavenumbers on the $200 \mathrm{mb}$ pressure surface at $38^{\circ} \mathrm{N}$ for the months of December thru March, plotted as a function of latitude. (Not evident because of the integration over wavenumber is the fact that zonal wavenumbers 4-7 dominate the flux.) The time-averaged zonal wind at this level and season is also shown as a heavy solid line. The zonal propagation of the eddies dominating the momentum flux is always westward with respect to the flow at this level (although typically eastward with respect to the ground), as we would expect from our simplest theory for Rossby waves.

Consider a system linearized about some zonal mean flow that is stirred with a particular space-time spectrum, localized in midlatitudes. Start with the barotropic vorticity equation for $2 \mathrm{D}$ flow on a $\beta$-plane, (ignoring spherical geometry for the moment)

$$
\frac{\partial \zeta}{\partial t}=-u \frac{\partial \zeta}{\partial x}-v \frac{\partial \zeta}{\partial y}-\beta v+S-D
$$




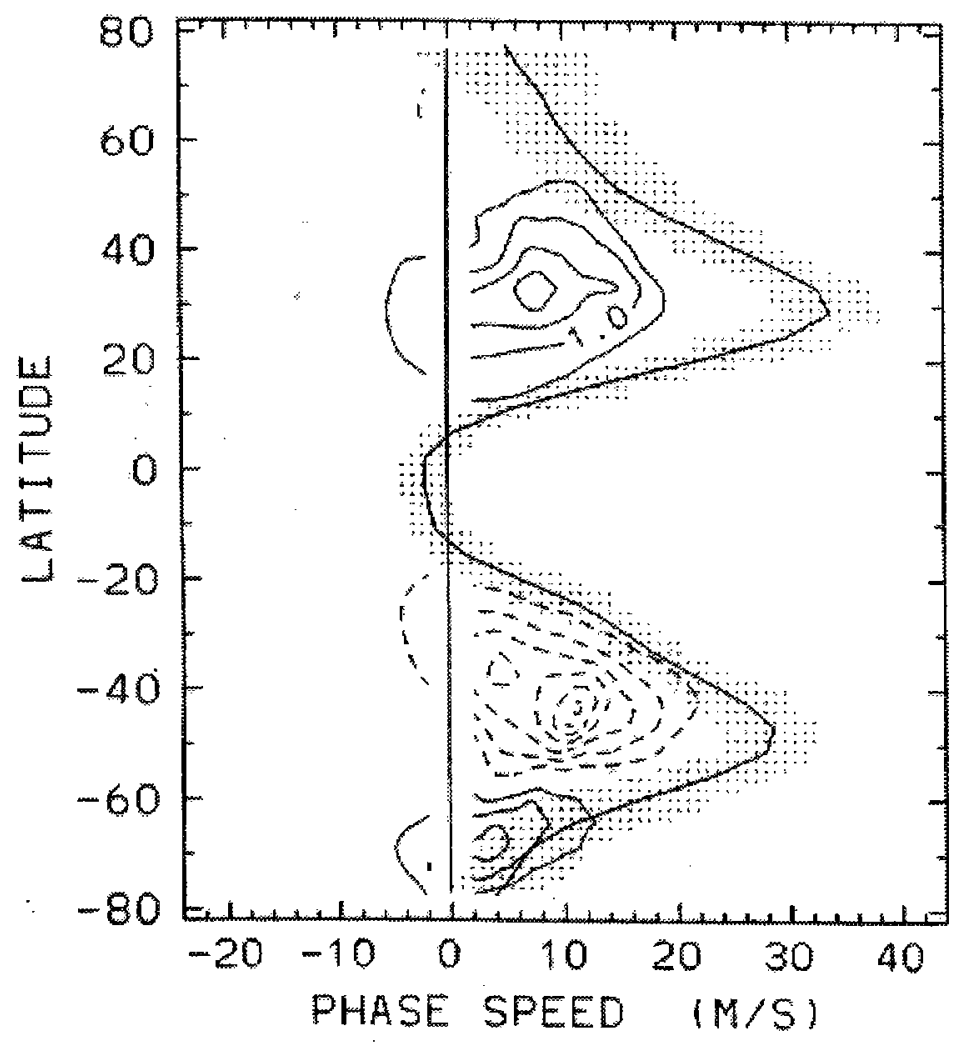

Figure 4: Phase speed spectrum of eddy momentum flux, $\overline{u^{\prime} v^{\prime}}$ at $200 \mathrm{mb}, 38^{\circ} \mathrm{N}$, averaged over Dec.-Mar., from [15]. Solid contours $\rightarrow$ northward flux; Dashed contours $\rightarrow$ southward flux. In the Northern hemisphere, the convergence into midlatitudes is almost entirely onesided (from the south); in the Southern hemisphere, there is some flux from the poleward side of the source region as well, implying significant poleward propagation 
Here $S$ is the stirring and $D$ is some unspecified damping, and

$$
\zeta=\frac{\partial v}{\partial x}-\frac{\partial u}{\partial y}=\nabla^{2} \psi
$$

is the relative vorticity of the flow, the zonal and meridional components of the horizontal velocity being

$$
(u, v)=\left(-\frac{\partial \psi}{\partial y}, \frac{\partial \psi}{\partial x}\right)
$$

Linearizing about the zonal flow $\bar{u}$, we have

$$
\frac{\partial \zeta^{\prime}}{\partial t}=-\bar{u} \frac{\partial \zeta^{\prime}}{\partial x}-\gamma v^{\prime}+S^{\prime}-D^{\prime}
$$

where

$$
\gamma=\beta-\frac{\partial^{2} \bar{u}}{\partial y^{2}}
$$

This stirring will generate waves that propagate away from the source, conserving their phase speed (on a sphere, their angular phase speed) and their zonal wavenumber. We can solve for each wavenumber and phase speed independently.

As the simplest case, suppose the background flow is uniform and neglect damping for the moment. Then, away from the source, for each space-time component the solution will simply be a plane Rossby wave,

$$
\psi=A \cos (k x+l y-\omega t)
$$

where $A$ is a constant, and $l$ must be determined, for given $k$ and $c$, so as to satisfy the familiar Rossby wave dispersion relation:

$$
\omega=c k=\bar{u} k-\frac{\beta k}{k^{2}+l^{2}} \equiv \Omega(k, l)
$$

There are two solutions for $l$ :

$$
l= \pm\left(\frac{\beta}{\bar{u}-c}-k^{2}\right)^{1 / 2}
$$

both of which are real, assuming that propagation is allowed (i.e., as long as the quantity within the square root is positive).

By substituting the plane-wave form for $\psi$ into the expressions for $u^{\prime}$ and $v^{\prime}$, one finds that the eddy momentum flux in this wave component is

$$
\overline{u^{\prime} v^{\prime}}=-A^{2} \frac{k l}{2}
$$

The product $k l$ determines the direction of the eddy momentum transport: if $k l<0$ then the eddy momentum flux is northward (positive $\overline{u^{\prime} v^{\prime}}$ ) and if $k l>0$ then the eddy momentum flux is southward (negative $\overline{u^{\prime} v^{\prime}}$ ).

The appropriate choice of signs to the north and south of the source can be determined from consideration of the meridional group velocity of a Rossby wave.

$$
G_{y}=\frac{\partial \Omega}{\partial l}=\frac{2 \beta k l}{\left(k^{2}+l^{2}\right)^{2}}
$$


The meridional group velocity has the sign of $k l$. The meridional flux of zonal momentum in Rossby waves is in the opposite direction to the group velocity. North of the source, we satisfy the radiation condition by choosing $k l>0$ so that $G_{y}$ is positive. South of the source we choose $k l<0$. The resulting Rossby waves converge positive zonal momentum into the source region from both directions, as we already know they must from the more general considerations in the first Lecture.

For those of unfamiliar with this use of a radiation condition in wave propagation problems, let's redo this computation in the presence of a small amount of damping of the form $D^{\prime}=\kappa \zeta^{\prime}$. Adding this damping is equivalent to adding a imaginary part to the frequency; outside of the source region, the plane wave solution must now satisfy

$$
\omega+i \kappa=\Omega(k, l)
$$

We can compute $l$ by assuming that it is close to one of the solutions to the inviscid dispersion relation $l=l_{0}+l_{1}$, with $\omega=\Omega\left(k, l_{0}\right)$. Using a Taylor expansion around $l_{0}$,

$$
\omega+i \kappa=\Omega\left(k, l_{0}\right)+\frac{\partial \Omega\left(k, l_{0}\right)}{\partial l} l_{1}
$$

From the definition of group velocity we have

$$
l_{1}=\frac{i \kappa}{G_{y}\left(k, l_{0}\right)}
$$

Substituted back into the original wave solution, the result is

$$
\tilde{\psi}=\operatorname{Re}\left[\exp \left(i l_{0} y-\frac{\kappa y}{G_{y}}\right)\right]
$$

In the presence of damping the solution must decay away from the source. Therefore, to the north (south) of the source we must choose the solution that results in positive (negative) $G_{y}$.

With $k l>0$ north of the source, lines of constant phase $(k x+l y=$ const $)$ are tilted $\mathrm{NW} / \mathrm{SE}$. South of the source, lines of constant phase are tilted NE/SW. The equatorward propagation and NE/SW tilt are predominant in our atmosphere, with most of the convergence of flux into midlatitudes coming from the equatorward side. If we add on the mean zonal flow, the result is the "tilted trough" structure familiar to meteorologists (see Figure 1).

If $\bar{u}$ is not uniform, the picture does not change if we can make a WKB approximation. Eventually, as a wave propagates equatorward, for example, it may reach a latitude at which its (angular) phase speed matches that of the zonal flow - beyond which point no Rossby wave propagation is possible. If WKB remains valid, $l$ increases rapidly as this point is approached, implying that the group velocity decreases, so that any damping that is present will have a long time to act and the wave will be dissipated. This argument is flawed, as one can show that WKB always breaks down before this "critical latitude" is reached. However, the conclusion is still correct - if linear theory is valid, and in the limit of infinitesimally small dissipation, the effects of dissipation are localized at each wave's critical latitude, where the waves are absorbed. We therefore expect waves with smaller 
phase speeds to propagate further into the tropics, as is clearly seen in the observations shown in Figure 4.

In this Rossby wave picture, the focus is on the momentum flux and the sign of the group velocity, whereas in the first lecture the focus was on the vorticity flux and Kelvin's circulation theorem, and no reference at all was made to a dispersion relation. We need to try to understand the relationship between these two arguments. For starters, we need to understand that the fluxes of (angular) momentum and vorticity are simply related. For our non-divergent flow (still ignoring spherical geometry) we have

$$
\zeta^{\prime} v^{\prime}=\left(\frac{\partial v^{\prime}}{\partial x}-\frac{\partial u^{\prime}}{\partial y}\right) v^{\prime}=\frac{1}{2} \frac{\partial v^{\prime 2}}{\partial x}-\frac{\partial u^{\prime} v^{\prime}}{\partial y}+u^{\prime} \frac{\partial v^{\prime}}{\partial y}=\frac{\partial}{\partial x}\left(\frac{1}{2}\left(v^{\prime 2}-u^{\prime 2}\right)\right)-\frac{\partial u^{\prime} v^{\prime}}{\partial y}
$$

Averaging over $x$ leaves

$$
\overline{\zeta^{\prime} v^{\prime}}=-\frac{\partial}{\partial y}\left(\overline{u^{\prime} v^{\prime}}\right)
$$

so that the eddy vorticity flux is the divergence of the eddy momentum flux.

The equation of motion in the $x$-direction is

$$
\frac{\partial u}{\partial t}=f v-\frac{\partial\left(u^{2}\right)}{\partial x}-\frac{\partial(u v)}{\partial y}-\frac{1}{\rho} \frac{\partial p}{\partial x}
$$

Zonally averaged we get

$$
\frac{\partial \bar{u}}{\partial t}=-\frac{\partial}{\partial y}\left(\overline{u^{\prime} v^{\prime}}\right)
$$

or, from (9),

$$
\frac{\partial \bar{u}}{\partial t}=\overline{v^{\prime} \zeta^{\prime}}
$$

where we have used the fact that $\bar{v} \equiv 0$

All of these results carry over to the sphere. For example. the spherical coordinate version of this relationship between vorticity and angular momentum fluxes is

$$
\overline{v^{\prime} \zeta^{\prime}} \cos (\theta)=-\frac{1}{a \cos (\theta)} \frac{\partial}{\partial \theta}\left(\cos ^{2}(\theta) \overline{u^{\prime} v^{\prime}}\right)
$$

The northward vorticity flux must sum to zero when integrated over latitude; in particular, if not identically zero, it must take on both positive and negative values.

Return to the linearized barotropic vorticity equation (5), multiply it by $\zeta^{\prime} / \gamma$ and average over $x$. The result is:

$$
\frac{\partial P}{\partial t}=-\overline{v^{\prime} \zeta^{\prime}}+\frac{\overline{S^{\prime} \bar{\zeta}^{\prime}}}{\gamma}-\frac{\overline{D^{\prime} \zeta^{\prime}}}{\gamma}
$$

where

$$
P \equiv \frac{1}{2 \gamma} \overline{\zeta^{\prime 2}}=\frac{\gamma}{2} \overline{\eta^{2}}
$$

The second form depends on the definition $\eta \equiv-\zeta^{\prime} / \gamma$. One can think of $\eta$ as the meridional parcel displacement that would create the perturbation vorticity, given the environmental vorticity gradient $\gamma . P$ is referred to as the pseudomomentum density of the waves. (Pseudomementum is, strictly speaking, $-P$, but we will ignore this!) Since the vorticity flux 
integrates to zero, the pseudomomentum integrated over the domain is conserved, in the absence of sources and sinks, and we can write the mean flow modification as

$$
\frac{\partial \bar{u}}{\partial t}=-\frac{\partial P}{\partial t}
$$

Consider a latitude circle at $y=y_{0}$ on which there are no eddies at $t=0$. At time $t$, when there are eddies present, we simply have $\overline{u(y, t)}-\overline{u(y, 0)}=-P(y, t)$.

If $\gamma>0, P$ is positive definite. When a wave enters a previously quiescent region, or, more precisely, if $P$ increases, the mean flow will decrease. This is a restatement of our previous results in this linear context, in which we see that $P$ is the measure of wave amplitude that is directly related to the mean flow modification.

If $\gamma$ is positive everywhere, simultaneous growth of the eddy throughout the domain (as in a normal mode instability) is precluded, for this would require $P$ to increase and the vorticity flux to be negative (downgradient) everywhere, which is impossible. This is why we specify external stirring to generate our eddies in this model; barotropic instability, resulting from a changes in sign of $\gamma$, is not the dominant process generating eddies in the upper troposphere.

To understand the expression for $P$, consider the fluid particles on a latitude circle, $y=y_{0}$, at time $t$. Look backwards in time, to $t=0$, and locate these same particles, which trace out a curve $y-y_{0}=\eta(x)$. For simplicity assume that this curve is gentle enough, as it would be for linear waves, that $\eta$ is a single valued function of $x$. The vorticity flux through this latitude, integrated from 0 to $t$, can simply be computed from the original vorticity distribution, by integrating the vorticity over the regions that end up moving through the latitude in question. If $\eta$ is small enough we get

$$
\overline{\int_{y_{0}}^{y_{0}+\eta}(\zeta(0)+\gamma y) d y}=\frac{\gamma}{2} \overline{\eta^{2}} .
$$

(Question: is this $\eta$ the same as that in the definition of $P$ above?) For further reading on pseudomomentum, see [1], [2]. The construction leading to (15) suggests one path towards generalizing the definition of $P$ to nonlinear disturbances.

The vorticity perturbation for a plane wave is $\left(-k^{2}+l^{2}\right) \psi^{\prime}$, so the pseudomomentum density is

$$
P=\frac{1}{4 \gamma}\left(k^{2}+l^{2}\right)^{2} A^{2}
$$

Using the expressions for the meridional group velocity of the Rossby wave (8) and the eddy momentum flux (7), we have the simple result

$$
-\overline{u^{\prime} v^{\prime}}=G_{y} P
$$

This relationship continues to be valid when the flow is slowly varying rather than constant.

In general, in the presence of forcing and dissipation in the wave equation,

$$
\frac{\partial P}{\partial t}=-\frac{\partial\left(-\overline{u^{\prime} v^{\prime}}\right)}{\partial y}+\text { sources }-\operatorname{sinks}
$$


In a statistically steady state, the eddy momentum flux convergence is determined by the sources and sinks of pseudomomentum.

Eddy energy, unlike pseudomomentum, is not conserved by this linear model, but rather the wave can exchange energy with the mean flow. Start with (10), multiply both sides by $\bar{u}$ and integrate over $y$,

$$
\frac{\partial}{\partial t} \int \frac{1}{2} \bar{u}^{2} d y=\int \overline{u^{\prime} v^{\prime}} \frac{\partial \bar{u}}{\partial y} d y
$$

Since the kinetic energy of the flow must be conserved, changes in zonal kinetic energy must be compensated by changes in eddy energy

$$
\frac{\partial}{\partial t} \int \frac{1}{2} \overline{u^{\prime 2}+v^{\prime 2}} d y=-\int \overline{u^{\prime} v^{\prime}} \frac{\partial \bar{u}}{\partial y} d y
$$

The globally integrated eddy kinetic energy decays as eddies propagate from regions of large zonal flow to regions of weaker flow, producing a countergradient flux of momentum. On the sphere, the meridional gradient of angular velocity plays the role of $\partial_{y} \bar{u}$ :

$$
\frac{\partial}{\partial t} \int \frac{1}{2} \overline{u^{\prime 2}+v^{\prime 2}} d y=-\int \overline{u^{\prime} v^{\prime}} \cos (\theta) \frac{\partial}{\partial \theta}\left(\frac{\bar{u}}{\cos (\theta)}\right) d \theta
$$

The term "negative viscosity" is sometimes encountered (less often in recent years, fortunately) when describing the poleward flux of momentum in the subtropics. It should be avoided. It is sometimes used simply as another term for the inverse energy cascade in $2 \mathrm{D}$ flows, and as such it is harmless but unnecessary. (The inverse energy cascade will be discussed later on in these lectures.) Transfer of energy to the zonal flow cannot, in general, be equated with transfer to larger scales. (A barotropic instability of a zonal flow transfers energy from the zonal mean to eddies while, like any 2D flow, moving energy to smaller total horizontal wavenumbers.) We have seen that the pattern of momentum fluxes is most easily understood as a consequence of downgradient vorticity fluxes (or, more properly, as we will see, potential vorticity fluxes). Whether the momentum fluxes are up or down the angular velocity gradient is somewhat incidental.

Note also that the dominant poleward eddy angular momentum fluxes in the subtropics are up the angular velocity gradient but down the angular momentum gradient; $\bar{u} / \cos (\theta)$ increases with increasing latitude but the angular momentum decreases. The latter becomes important when we discuss the possibility of equatorial superrotation later in these lectures.

We need to know the sinks as well as the sources of pseudomomentum to understand the distribution of surface stress in midlatitudes. In the upper tropospheric layer under consideration, we are generally far removed from any direct effects of boundary layer turbulence, so "dissipation" of waves in this context should be thought of as a consequence of "wave breaking". If Rossby waves are large enough they cause the vorticity contours to overturn in the horizontal plane, causing a cascade of vorticity variance to small scales where it is eventually dissipated. Consider a wave that produces eddy zonal winds of magnitude $u^{\prime}$ at a particular latitude. Suppose that the wave amplitude is steady. Move to a reference frame translating with the wave so that the total flow is independent of time and so that streamlines, vorticity contours and particle trajectories all coincide. Being a Rossby wave, the mean flow in this reference frame, $\bar{u}-c$ is positive. But if

$$
u^{\prime}>\bar{u}-c
$$


in part of the wave, streamlines and vorticity contours will overturn. Since the wave is not steady in reality, these overturning contours will result in wave breaking, i.e, mixing. If the wave is infinitesimal, it will break only when it reaches its critical latitude, where $\bar{u}=c$. More realistically, it will break before it reaches this latitude, by an amount dependent on the wave amplitude. One can sense this kind of behavior in Figure 5 above.

\section{A shallow water model}

So far, we have used a non-divergent two-dimensional model in our discussions. We now describe a model for fluid of finite depth flowing over topography. This system provides a simple framework for introducing some important concepts.

Consider a layer of homogeneous, inviscid and incompressible fluid with a rigid lid at $z=H_{0}$ and a rigid undulating lower boundary defined by $z=h(x, y)$. The thickness of the layer is $H(x, y)=H_{0}-h(x, y)$. The thickness perturbation is $H^{\prime}(x, y)=-h(x, y)$. The layer of fluid is assumed to have a small aspect ratio, i.e., the average depth $H_{0}$ of the layer is small compared with the horizontal length scale of the fluid motion. In this case, the vertical acceleration of the fluid can be neglected and we can make the hydrostatic approximation

$$
\frac{\partial p}{\partial z}=-\rho_{0} g
$$

where $\rho_{0}$ is the constant density of the fluid. It follows by integrating from the top down that the horizontal pressure gradient is independent of depth, and equal to the pressure gradient on the rigid lid, so we can look for solutions for which the horizontal velocity is also independent of depth. The vertical velocity varies linearly with depth from its value at the lower boundary $w=\mathbf{v} \cdot \nabla h$ to zero at the top.

The equations that result from making the above assumptions are the shallow water equations, which we are specializing here to the case of a rigid lid. Kelvin's circulation theorem remains valid (although consistent with the hydrostatic approximation we ignore the contribution of vertical motion to the circulation.) As before, using Stokes theorem for an infinitesimal material loop with area $\delta A$,

$$
\frac{D}{D t}(\omega \cdot \hat{\mathbf{n}} \delta A)=\frac{D}{D t}\left(\frac{\omega \cdot \hat{\mathbf{n}}}{H}(H \delta A)\right)=0
$$

Note that $H \delta A$ is the mass of the vertical column of fluid whose cross section is defined by the surface element $\delta A$; this mass must be conserved, so

$$
\frac{D}{D t}\left(\frac{\omega \cdot \hat{\mathbf{n}}}{H}\right)=\frac{D}{D t}\left(\frac{f+\zeta}{H}\right)=0
$$

The potential vorticity

$$
Q \equiv \frac{f+\zeta}{H}
$$

is conserved following the flow.

We now make the quasi-geostrophic approximation, based on the assumptions that the flow is on a $\beta$-plane with $f=f_{0}+\beta y$ and $\beta y<<f_{0}$, that the vorticity of the motion relative 
to solid body rotation is small compared to the vorticity of the solid body itself, $\zeta<<f_{0}$ and that the amplitude of the perturbation height is small compared with the thickness of the layer $h<<H$. We can then expand $Q$ in these three small parameters and obtain:

$$
Q \approx \frac{f_{0}}{H_{0}}+\frac{1}{H_{0}}\left(\beta y+\zeta+\frac{f_{0} h}{H_{0}}\right)
$$

We define

$$
q=\beta y+\zeta+\frac{f_{0} h}{H_{0}}
$$

The effect of topography is now embedded in the conservation law for $q$ :

$$
\frac{\partial q}{\partial t}=-u \frac{\partial q}{\partial x}-v \frac{\partial q}{\partial y}
$$

The fact that the flow is non-divergent to first approximation, due to assumptions of small Rossby number and $\beta y \ll f_{0}$, allows us to define a streamfunction $\psi$, as in the non-divergent case:

$$
\left(-\frac{\partial \psi}{\partial y}, \frac{\partial \psi}{\partial x}\right)=(u, v)
$$

The equation for the evolution of the zonally averaged flow in a shallow water model can be obtained by starting with the horizontal momentum equation:

$$
\frac{\partial u}{\partial t}=f v-u \frac{\partial u}{\partial x}-v \frac{\partial u}{\partial y}-\frac{1}{\rho} \frac{\partial p}{\partial x}
$$

or

$$
\left.\frac{\partial u}{\partial t}=(f+\zeta) v-\frac{\partial}{\partial x}\left(\frac{p}{\rho_{0}}+\frac{1}{2}\left(u^{2}+v^{2}\right)\right)\right)
$$

Averaging zonally, we have

$$
\frac{\partial \bar{u}}{\partial t}=\overline{(f+\zeta) v}
$$

With the small Rossby number approximation of QG theory this reduces to

$$
\frac{\partial \bar{u}}{\partial t}=f_{0} \bar{v}+\overline{v^{\prime} \zeta^{\prime}}=f_{0} \bar{v}-\frac{\partial \overline{u^{\prime} v^{\prime}}}{\partial y}
$$

The replacement of the vorticity flux by the momentum flux convergence is exact if the flow is non-divergent. We can still make this substitution within the quasi-geostrophic approximation because the flow is non-divergent to lowest order.

Since $f_{0}$ is large compared to the relative vorticity, the meridional advection of the vorticity of solid body rotation by the (ageostrophic) mean meridional flow must be retained. It is important that $\bar{v}$ need not be zero, even though the fluid is confined between rigid top and bottom boundaries. Mass conservation does tell us that $\partial_{y}(\overline{v H})$ must be zero, since we cannot converge mass into any region with rigid lids. Assuming that the mass flux vanishes at northern or southern boundaries, $\overline{v H}$ must vanish everywhere, so that

$$
\overline{v H}=\bar{v} \bar{H}+\overline{v^{\prime} \bar{H}^{\prime}}=0
$$


So $\bar{v}$ can be non-zero if the eddy north-south flow is correlated with the thickness of the layer.

Linearizing the potential vorticity equation about a zonally symmetric mean state as before, we have

$$
\frac{\partial q^{\prime}}{\partial t}=-\bar{u} \frac{\partial q^{\prime}}{\partial x}-v^{\prime} \frac{\partial \bar{q}}{\partial y}-D
$$

where the term $D$ has been added to represent dissipation. We do not force the eddies with external stirring anymore - the zonally asymmetric topography is the source of the eddies. On multiplying by $q^{\prime}$ and taking the zonal average, we get

$$
\frac{\partial P}{\partial t}=\frac{\partial}{\partial t}\left(\overline{\frac{q^{\prime 2}}{2 \gamma}}\right)=-\overline{v^{\prime} q^{\prime}}-\overline{D q^{\prime}} / \gamma
$$

where $\gamma \equiv \partial_{y} \bar{q}$. This is the pseudomomentum conservation law for this system. In a statistically steady state, the value of the potential vorticity flux is determined by the dissipation; in the regions where $D=0, \overline{v^{\prime} q^{\prime}}$ must be zero as well.

The eddy flux of potential vorticity is

$$
\overline{v^{\prime} q^{\prime}}=\overline{v^{\prime} \zeta^{\prime}}-\frac{f_{0} \overline{v^{\prime} H^{\prime}}}{H_{0}}
$$

This flux is comprised of two parts: the vorticity flux $\overline{v^{\prime} \zeta^{\prime}}$; and the contribution from the mass flux $\overline{v^{\prime} H^{\prime}}$. In a statistically steady state, and in the absence of dissipation, the sum of the two terms on the RHS must vanish. The individual terms in this expression need not vanish.

Now consider the situation in which the orography is confined to a particular zone of latitudes. Rossby waves will be generated by the flow over this orography and propagate meridionally away from this zone, into the region where the lower boundary is flat. Assume that the situation is statistically steady. Assume also that the wave amplitudes and horizontal shears are such that there is no wave breaking in the region of the orography; rather the waves break outside of this region. Therefore, the potential vorticity flux vanishes in the source region, but is negative in the region of wave dissipation far from the source. As we have seen, Rossby waves radiating away from a source region transport zonal momentum into this region. Therefore, within the source region, the momentum flux convergence must be balanced by an eddy mass flux:

$$
\frac{f_{0} \overline{v^{\prime} H^{\prime}}}{H_{0}}=-\frac{\partial \overline{u^{\prime} v^{\prime}}}{\partial y}
$$

The eddy mass flux must be poleward above the topography. Since there can be no total mass flux, the mass transport by the "mean meridional circulation", $\bar{v}$, must be equatorward just as in the upper tropospheric branch of the Ferrell cell.

This is not a coincidence - this is the essence of the Ferrell cell! Once again we need to consider this flow as a model of an upper tropospheric layer of fluid, forced by undulations in its lower boundary. Note first that nothing in the argument depends on the undulations being fixed in time. More precisely, as long as the zonal mean of the orography does not 
change, everything in this argument carries through unmodified. If the zonal means do change in time, then we need only add on the meridional mass transport required by mass conservation.

We can write the mean flow acceleration in terms of the potential vorticity flux by adding and subtracting the mass flux:

$$
\frac{\partial \bar{u}}{\partial t}=f_{0} \bar{v}+\frac{f_{0}}{H_{0}} \overline{v^{\prime} H^{\prime}}+\overline{v^{\prime} q^{\prime}}=f_{0} \overline{v^{*}}+\overline{v^{\prime} q^{\prime}}
$$

The residual circulation $\bar{v}^{*}$ is defined so as to be proportional to the total mass flux:

$$
\bar{v}^{*}=\frac{\overline{v H}}{H_{0}}=\bar{v}+\overline{\frac{v^{\prime} H^{\prime}}{H_{0}}}
$$

As we have already seen, from mass conservation in this simple rigid lid system, $\bar{v}^{*}=0$.

It is interesting, and fundamental, that in this simple system it is the potential vorticity flux, rather than the vorticity flux, that determines the zonal acceleration of the mean flow. One way of thinking about this is to start with the zonal mean potential vorticity equation:

$$
\frac{\partial \bar{q}}{\partial t}=-\frac{\partial \overline{v^{\prime} q^{\prime}}}{\partial y}
$$

Since the rigid lids require that the mean thickness of each layer is unchanged,

$$
\frac{\partial \bar{q}}{\partial t}=-\frac{\partial^{2} \bar{u}}{\partial t \partial y}
$$

One integration in latitude then yields, as before,

$$
\frac{\partial \bar{u}}{\partial t}=\overline{v^{\prime} q^{\prime}}=\overline{v^{\prime} \zeta^{\prime}}-\frac{f_{0} \overline{v^{\prime} H^{\prime}}}{H_{0}}
$$

The final term of the RHS of this expression has an important physical interpretation: it is (the quasi-geostrophic approximation to) the force that the topography exerts on the fluid. The pressure force exerted by the atmosphere on the surface is normal to the boundary, so it has a zonal component when the surface has an east-west slope. If the slope is $\partial_{x} h \equiv \tan (\phi)$, then the eastward component of the pressure force is $p \sin (\phi)$. Making the small angle approximation (inherent in the shallow water model), we can ignore the difference between sin and tan so that the zonal component, averaged around a latitude circle, is

$$
\overline{p_{s} \frac{\partial h}{\partial x}}=-\overline{h \frac{\partial p_{s}}{\partial x}}
$$

where $p_{s}$ is the surface pressure, related to the interior pressure by the hydrostatic relation:

$$
p_{s}=p+\rho_{0} g(z-h)
$$

Since

$$
\overline{h \frac{\partial h}{\partial x}} \equiv 0
$$


we can replace $p_{s}$ by $p$, the pressure at a fixed height, in the expression for the zonal force on the surface. We can then use geostrophy,

$$
\frac{\partial p^{\prime}}{\partial x}=f_{0} \rho_{0} v^{\prime}
$$

to rewrite the force as

$$
-f_{0} \rho_{0} \overline{v^{\prime} h}=f_{0} \rho_{0} \overline{v^{\prime} H^{\prime}}
$$

The force exerted by the surface on the atmosphere is equal in magnitude and opposite in sign. Dividing this force per unit horizontal area by the mass per unit area, $\rho_{0} H_{0}$ to get an acceleration we find the desired expression.

$$
\frac{\partial \bar{u}}{\partial t}=\ldots . .-\frac{f_{0}}{H_{0}} \overline{v^{\prime} H^{\prime}}
$$

When localized topography forces waves that propagate away from the region of excitation without being dissipated in this region, the resulting eddy momentum flux convergence is exactly balanced by the deceleration of the flow by the "form drag", or "mountain torque" exerted by the topography on the atmosphere! It is vitally important here that the "stirring" creating the eddies is not, as in Lectures 1 and 2, an externally specified vorticity source; the vorticity source here, the stretching associated with flow up and down the surface topography, is flow dependent. It is striking that this flow dependence produces this non-acceleration result for steady, inviscid waves. Potential vorticity dynamics is clearly the simplest way of seeing where this result comes from.

\section{A Two Layer Model}

Two layer models are a classical and natural starting point for thinking about the baroclinic dynamics of the troposphere. We choose to consider two isentropic layers of a compressible ideal gas, to make our model a bit more meteorological, rather than the more traditional two incompressible layers with fixed densities.

Evolution in the troposphere is sufficiently slow as compared to the time scales at which local thermodynamic equilibrium is maintained that we can assume that the entropy of a fluid parcel is conserved except for explicit diabatic effects, the most important of which is radiative heating. (We continue to consider a dry model.) The entropy per unit mass, $s$, of an ideal gas, with the equation of state $p=\rho R T$, can be written as

$$
s=c_{p} \ln (\Theta) ; \Theta \equiv T / \Pi ; \Pi \equiv\left(\frac{p}{p_{*}}\right)^{\kappa} ; \kappa \equiv \frac{R}{c_{p}} .
$$

Here $T$ is the temperature, $\Theta$ the potential temperature, $p_{*}$ is a reference pressure, and $c_{p}$ the heat capacity at constant pressure.

Figure 5 shows the observed zonal mean isentropes in the troposphere. The potential temperature increases with height, at about $3 \mathrm{~K} / \mathrm{km}$, indicating gravitational stability to dry convection. Given this vertical stability and the decrease in temperature with increasing latitude, isentropic surfaces tilt upwards with latitude in the troposphere. It is interesting that, roughly speaking, this slope is such that it takes one from the surface in the tropics 
to the tropopause in high latitudes. That is, the potential temperature difference between equator and pole is roughly the same as that between the surface and the tropopause. Is this a coincidence?
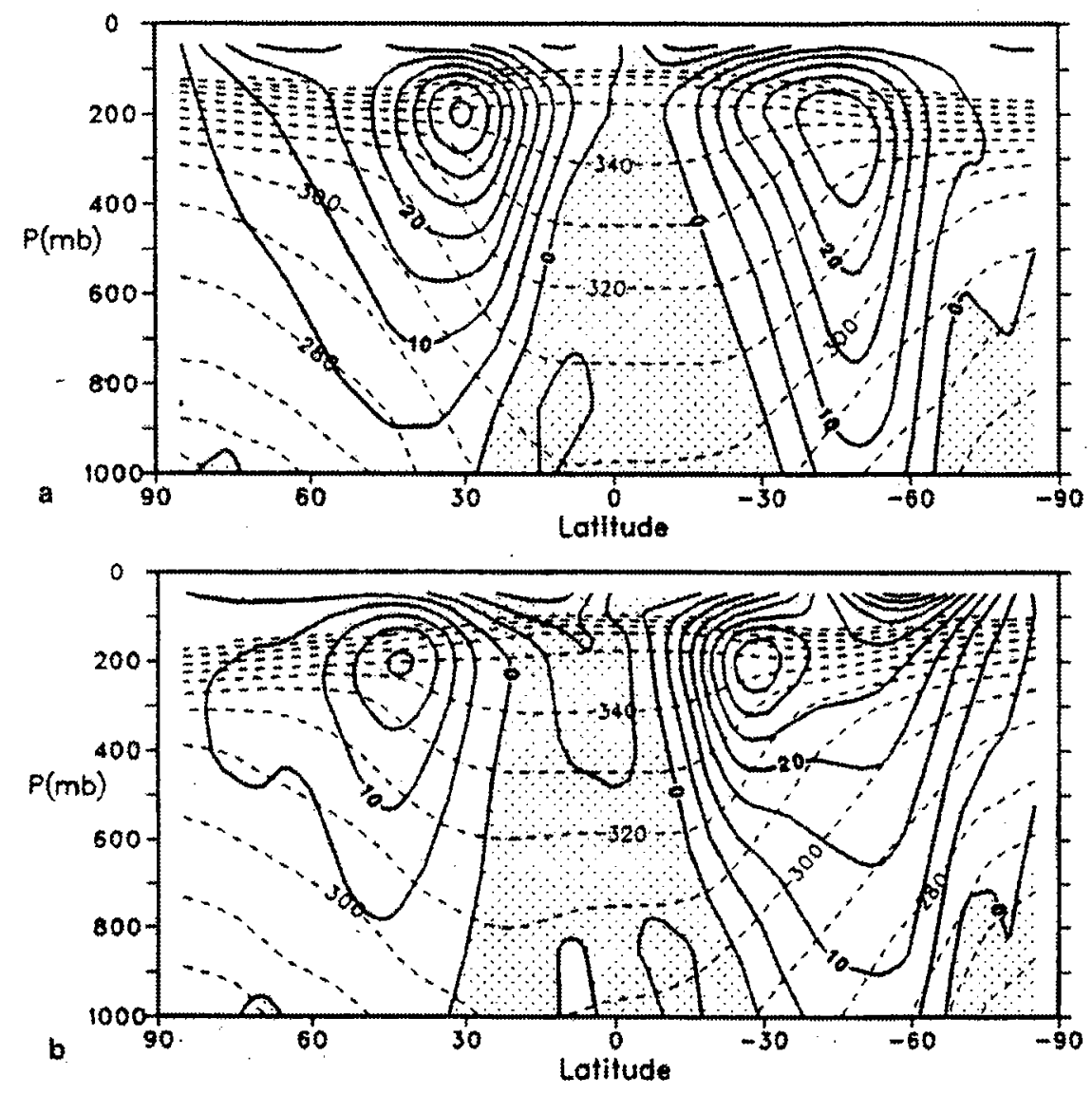

Figure 5: Observed zonal mean isentropes (dashed) and zonal winds (solid). The upper panel is DJF; the lower panel JJA. from [8]

We continue to focus on midlatitudes, and idealize the troposphere as consisting of two isentropic layers separated by one interface. We work in pressure coordinates, and speak of the pressure, rather than the height, of this interface. We allow the pressure at the surface (assumed to be at constant height) to vary, but we fix the pressure at the top of the upper layer. The subscripts 1 and 2 refer to the upper and lower layers respectively. The two potential temperatures are $\Theta_{1}$ and $\Theta_{2}$, with $\Theta_{1}>\Theta_{2} . p_{B}, p_{M}$, and $p_{T}$ are, respectively, the pressures at the bottom of the lower layer, the interface between the layers, and the top of the upper layer.

The hydrostatic approximation for an ideal gas is

$$
\frac{\partial \Phi}{\partial \Pi}=-c_{p} \Theta
$$

where $\Phi$ is the geopotential. If we integrate from the bottom upwards we get in lower and 
upper layers, respectively,

$$
\begin{aligned}
& \Phi_{2}=c_{p} \Theta_{2}\left(\Pi_{B}-\Pi\right) \\
& \Phi_{1}=c_{p} \Theta_{2}\left(\Pi_{B}-\Pi_{M}\right)+c_{p} \Theta_{1}\left(\Pi_{M}-\Pi\right)
\end{aligned}
$$

so that, computing the gradient at fixed pressure

$$
\begin{aligned}
\nabla \Phi_{2} & =c_{p} \Theta_{2} \nabla \Pi_{B} \\
\nabla \Phi_{1} & =c_{p} \Theta_{2} \nabla \Pi_{B}+c_{p}\left(\Theta_{1}-\Theta_{2}\right) \nabla \Pi_{M}
\end{aligned}
$$

These gradients are independent of pressure within each layer, so we can look for solutions in which the horizontal flow is independent of pressure, analogous to shallow water theory.

Geostrophic motion in pressure coordinates takes the form

$$
f(u, v)=\left(-\frac{\partial \Phi}{\partial y}, \frac{\partial \Phi}{\partial x}\right)
$$

So the thermal wind equation for the zonal flow, for example, is given by

$$
f\left(u_{1}-u_{2}\right)=-c_{p}\left(\Theta_{1}-\Theta_{2}\right) \frac{\partial \Pi_{M}}{\partial y}
$$

In the context of this two layer model, radiative heating can be thought of as converting some of the mass of the lower layer into mass of the upper layer, that is, as lowering the interface. Radiative cooling raises the interface. Keeping in mind that fixing the static stability is somewhat artificial in this context, radiative fluxes can be modeled by relaxing the interface to some steep "radiative equilibrium" value. It is the competition between the steepening due to the radiation drive and the shallowing of the interface due to the circulation that we are interested in. It should be clear from the start that the circulation must transport mass poleward in the upper layer, and equatorward in the lower layer, if it is to maintain a slope that is less steep than "radiative equilibrium".

Kelvin's circulation theorem remains valid for an inviscid ideal gas as long as the material circuit is confined to an isentropic surface. This centrally important result is responsible, to a great extent, for the utility of isentropic analyses in the atmosphere. In our case, we need only restrict ourselves to circuits that reside entirely in one or the other layer. Just as for the shallow water model of Lecture 3, we have

$$
\frac{D}{D t}(\omega \cdot \hat{\mathbf{n}} d A)=0
$$

We use the notation

$$
H \equiv \frac{\Delta p}{g}
$$

for the mass per unit area of a layer, where $\Delta p$ is the pressure thickness of the layer $\left(p_{T}-p_{M}\right.$ in the upper layer and $p_{M}-p_{B}$ in the lower layer). Conservation of mass

$$
\frac{D}{D t}(H d A)=0
$$


implies

$$
\frac{D}{D t}\left(\frac{f+\zeta}{H}\right)=0
$$

just as before. Similarly, under the quasi-geostrophic approximation, in each layer,

$$
q=\beta y+\zeta-\frac{f_{o} H}{H_{o}}
$$

Also the mean zonal acceleration in each layer is again given by $\overline{v(f+\zeta)}$, and the same manipulations, using quasi-geostrophic scaling, yields the mean momentum equation in each layer in the "Transformed Eulerian Mean" form ([1], [2]).

$$
\begin{aligned}
& \frac{\partial \overline{u_{1}}}{\partial t}=f v_{1}^{*}+\overline{v_{1}^{\prime} q_{1}^{\prime}} \\
& \frac{\partial \overline{u_{2}}}{\partial t}=f v_{2}^{*}+\overline{v_{2}^{\prime} q_{2}^{\prime}}-\kappa \overline{u_{2}} .
\end{aligned}
$$

We have now included frictional drag in the lower layer, with $\kappa$ being a linear drag coefficient. The northward mass flux in each layer is $\overline{v_{i}^{\prime} H_{i}^{\prime}} \equiv H_{0 i} v_{i}^{*}$.

The QG potential vorticity flux can again be written as a sum of a vorticity flux, or momentum flux convergence, and a mass flux. In the lower layer, for example,

$$
\overline{v_{2}^{\prime} q_{2}^{\prime}}=\overline{v_{2}^{\prime} \zeta_{2}^{\prime}}-\frac{f_{o}}{H_{o, 2}} \overline{v_{2}^{\prime} H_{2}^{\prime}}
$$

Focusing on the thickness flux,

$$
\overline{v_{2}^{\prime} H_{2}^{\prime}}=\frac{\overline{v_{2}^{\prime}\left(p_{B}^{\prime}-p_{M}^{\prime}\right)}}{g}
$$

By geostrophy

$$
\overline{v_{2}^{\prime} p_{B}^{\prime}}=\frac{c_{p} \Theta_{2}}{f} \frac{\overline{\partial \Pi_{B}^{\prime}}}{\partial x} p_{B}^{\prime}
$$

However, $\Pi_{B}$ is simply a function of $p_{B}$, so $\overline{v_{2}^{\prime} p_{B}^{\prime}}=0$ and

$$
\overline{v_{2}^{\prime} H_{2}^{\prime}}=-\frac{\overline{v_{2}^{\prime} p_{M}^{\prime}}}{g}
$$

Meanwhile, in the upper layer,

$$
\overline{v_{1}^{\prime} H_{1}^{\prime}}=\frac{\overline{v_{1}^{\prime}\left(p_{M}^{\prime}-p_{T}^{\prime}\right)}}{g}=\frac{\overline{v_{1}^{\prime} p_{M}^{\prime}}}{g},
$$

since $p_{T}$ is assumed to be constant. Finally, by the thermal wind relation,

$$
\overline{\left(v_{1}^{\prime}-v_{2}^{\prime}\right) p_{M}^{\prime}}=\overline{\frac{\partial F\left(p_{M}^{\prime}\right)}{\partial x} p_{M}^{\prime}}=0
$$

where $F$ is a function of $p_{M}$ so that

$$
\overline{v_{1}^{\prime} p_{M}^{\prime}}=\overline{v_{2}^{\prime} p_{M}^{\prime}}
$$


and

$$
\overline{v_{1}^{\prime} H_{1}^{\prime}}=-\overline{v_{2}^{\prime} H_{2}^{\prime}} \text {. }
$$

The eddy mass fluxes in the two layers cancel. It might appear that we could have obtained this directly from mass conservation, but mass conservation only tells us that, in a steady state, the total mass fluxes in the two layers must cancel. Given this relation between the eddy fluxes, we know that the mean mass fluxes must also be equal and opposite in a steady state.

This result can once again be related to the form drag exerted by one layer on the other layer. Given an interface with height $z_{M}$, or slope $\partial_{x} z_{M}$, the drag per unit mass exerted on the lower layer by the upper layer is

$$
F=\frac{1}{H_{o, 2}} \overline{p_{m}^{\prime} \frac{\partial z_{M}}{\partial x}}=\frac{1}{g H_{o, 2}} \overline{p_{M}^{\prime} \frac{\partial \phi_{M}}{\partial x}}
$$

Using geostrophy

$$
f v_{2}^{\prime}=c_{p} \Theta_{2} \frac{\partial \Pi_{B}^{\prime}}{\partial x}
$$

and the expression for $\Phi_{M}$

$$
\Phi_{M}=c_{p} \Theta_{2}\left(\Pi_{B}-\Pi_{M}\right)
$$

this yields

$$
F=\frac{f_{o}}{g H_{o, 2}} \overline{v_{2}^{\prime} H_{2}^{\prime}}
$$

Thus, the statement that $\overline{v_{1}^{\prime} H_{1}^{\prime}}=-\overline{v_{2}^{\prime} H_{2}^{\prime}}$ is, within the quasi-geostrophic approximation, a statement of Newton's Third Law.

Recall that in a non-divergent barotropic model the northward eddy vorticity flux must sum to zero when integrated over latitude. Using our result that the eddy fluxes of mass cancel in the two layers, and that the vorticity flux is the convergence of the eddy momentum flux in each layer, we have, as a generalization, that

$$
\sum_{i=1}^{2} \int H_{o, i} \overline{v_{i}^{\prime} q_{i}^{\prime}} d y=0,
$$

where now we must sum over the two layers as well as integrate over latitude.

Because potential vorticity is conserved following the flow, linear conservative eddies that are growing must transport potential vorticity down the mean gradient. That is, multiplying

$$
\frac{\partial q^{\prime}}{\partial t}=-\bar{u} \frac{\partial q^{\prime}}{\partial x}-v^{\prime} \frac{\partial \bar{q}}{\partial y}
$$

by $q^{\prime}$ and averaging zonally,

$$
\frac{1}{2} \frac{\partial \overline{q^{\prime 2}}}{\partial t}=-\overline{v^{\prime} q^{\prime}} \frac{\partial \bar{q}}{\partial y}
$$

Coupled with the fact that the potential vorticity flux integrates to zero over the domain, this results in the Charney-Stern-Pedlosky criterion that the mean potential vorticity gradient must take on both signs if there is to be simultaneous growth everywhere, as in a 
normal mode instability. For nonlinear eddies, the variance of potential vorticity can be advected from one latitude to another, so the local relationship between eddy growth and downgradient flux can be broken:

$$
\frac{1}{2} \frac{\partial \overline{q^{\prime 2}}}{\partial t}=-\overline{v^{\prime} q^{\prime}} \frac{\partial \bar{q}}{\partial y}-\frac{1}{2} \frac{\partial \overline{v^{\prime} q^{\prime 2}}}{\partial y}
$$

But integrating in $y$ this nonlinear term does not contribute

$$
\frac{1}{2} \frac{\partial}{\partial t} \int \overline{q^{\prime 2}} d y=-\int \overline{v^{\prime} q^{\prime}} \frac{\partial \bar{q}}{\partial y} d y
$$

In a statistically steady state, the production of variance due to downgradient fluxes is balanced by the loss of variance resulting from a cascade to small scales where dissipative processes act. If we assume that a) all (or most, at least) nonconservative effects in the large-scale potential vorticity equation are dissipative, then the eddy potential vorticity flux must be downgradient on average. If, in addition, we assume that $b$ ) the advection of variance is small, then the flux must be downgradient at all latitudes. While there are a couple of assumptions required, the local downgradient restriction is a very useful one when developing models of the zonally averaged eddy potential vorticity flux. (Without zonal averaging, in a zonally asymmetric climate, the advection of eddy variance by the mean flow becomes much more important, and the problem is more complicated.)

Since the eddy fluxes cannot be everywhere of the same sign, given locally downgradient fluxes the zonal mean potential vorticity gradient cannot be everywhere positive. This is a (crude) generalization of the Charney-Stern-Pedlosky criterion to nonlinear statistically steady states, requiring the two assumptions a) and b) above.

The mean potential vorticity gradients are given by:

$$
\frac{\partial \overline{q_{i}}}{\partial y}=\beta-\frac{\partial^{2} \overline{u_{i}}}{\partial y^{2}}-\frac{f_{o}}{H_{o, i}} \frac{\partial H_{i}}{\partial y} .
$$

Either the horizontal curvature or the thickness gradient must overcome $\beta$. If horizontal curvature is the primary reason for the sign change, we speak of barotropic eddy production; if it is the thickness gradient we speak of baroclinic production. In the troposphere, baroclinic eddy production is the dominant process. If the slope of the interface is sufficiently steep, the thickness gradient in the lower layer will overcome $\beta$, and the lower layer potential vorticity gradient will be negative. In a statistically steady state, we then expect poleward potential vorticity fluxes in the lower layer and equatorward fluxes in the upper layer. These potential vorticity fluxes are dominated by the eddy thickness fluxes, as the form drag at the interface transfers zonal momentum from the upper to the lower layer.

The acceleration due to the upper layer flux requires, in a steady state, a mass flux, i.e., a residual circulation $v^{*}$, that is poleward, so that the Coriolis force on this circulation balances the torque due to the potential vorticity flux (primarily the form drag). The residual circulation in the lower layer must carry the same mass flux equatorward. This circulation is consistent with the idea that the mass fluxes associated with the atmospheric circulation will be such as to fight the steepening due to the radiative driving.

In the lower layer, the mass transport is in the opposite direction to the Ekman drift associated with the surface westerlies, ie., it is opposite to the direction of the Ferrel cell. 
As eddy momentum fluxes are small in the lower troposphere, this is equivalent to saying that the form drag on the upper surface of the lower layer, which is accelerating the zonal winds in that layer, must be larger than the surface drag, which is decelerating these winds. For the mass flux to be opposite in sign to the mean meridional circulation, it must be the case that the eddy mass flux (proportional to the form drag) is not only opposite in sign to the mass flux due to the mean circulation, but also larger in amplitude.

The Ferrel cell disappears when one considers mass fluxes rather than averages at constant height, pressure, or entropy. (The Ferrel cell is still present when averaging at constant entropy, despite the statements one sometimes hears to the contrary; it is the mass weighting that is crucial.) But this does not mean that the midlatitude overturning merges smoothly into the tropical overturning in the Hadley cell. Figure 6 is a plot of the mass flux, in the (entropy, latitude) plane computed from a GCM climate simulation for January. The atmosphere is divided into a large number of isentropic layers and the poleward mass flux is computed in each one; the cross-isentropic mass flux, due to radiation and latent heat release, is then computed from mass conservation. Also shown is the mean surface potential temperature. The key point is that the tropical poleward mass flux, ending at the latitude of the subtropical jet, occurs within very different isentropic layers than the shallower midlatitude overturning. The Hadley cell still has a well-defined meridional extent despite the fact that the flow in the upper troposphere is everywhere polewards.

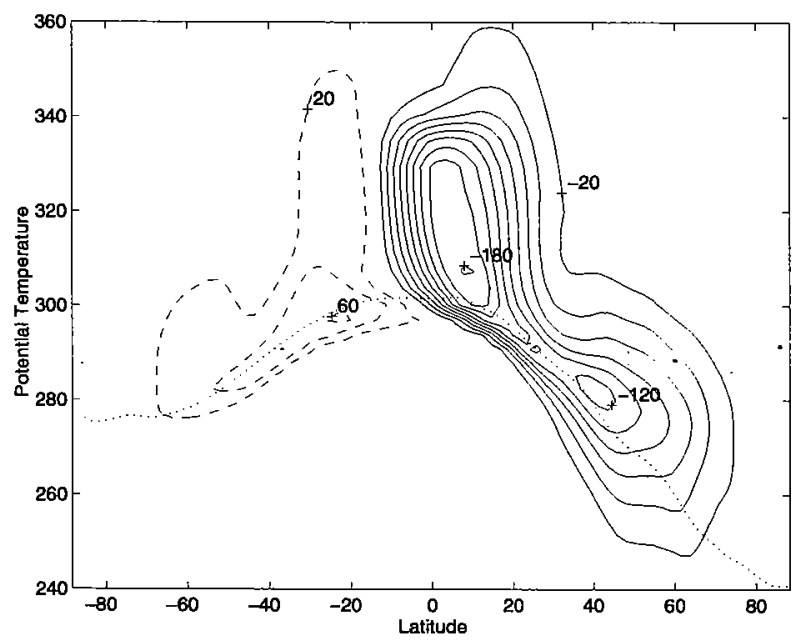

Figure 6: The mass transport streamfunction as a function of potential temperature and latitude, in January, as computed from a GCM, from [6]. The dotted line is the median surface temperature.

If we sum the zonal mean equations of motion in the two layers, in their Transformed Eulerian form, assume a steady state, and use the fact that the vertically integrated poleward mass flux must be zero,

$$
v_{1}^{\star} H_{o, 1}+v_{2}^{\star} H_{o, 2}=0 .
$$

we have

$$
H_{o, 1} \overline{v_{1}^{\prime} q_{1}^{\prime}}+H_{o, 2} \overline{v_{2}^{\prime} q_{2}^{\prime}}=\kappa \overline{u_{2}} H_{o, 2}
$$


The lower layer wind is determined by the vertical integral of the eddy potential vorticity fluxes.

Suppose, as is reasonable, that the potential vorticity fluxes are everywhere equatorward in the upper layer and poleward in the lower layer. The magnitude of the fluxes must be equal when integrated over latitude, but in order to produce surface westerlies in midlatitudes, we must now require the lower layer flux to be more sharply peaked in midlatitudes, and the upper layer flux to be more broadly distributed. Figure 7 shows this situation schematically, along with the opposite case, with a more broadly distributed lower layer flux that would produce midlatitude surface easterlies!

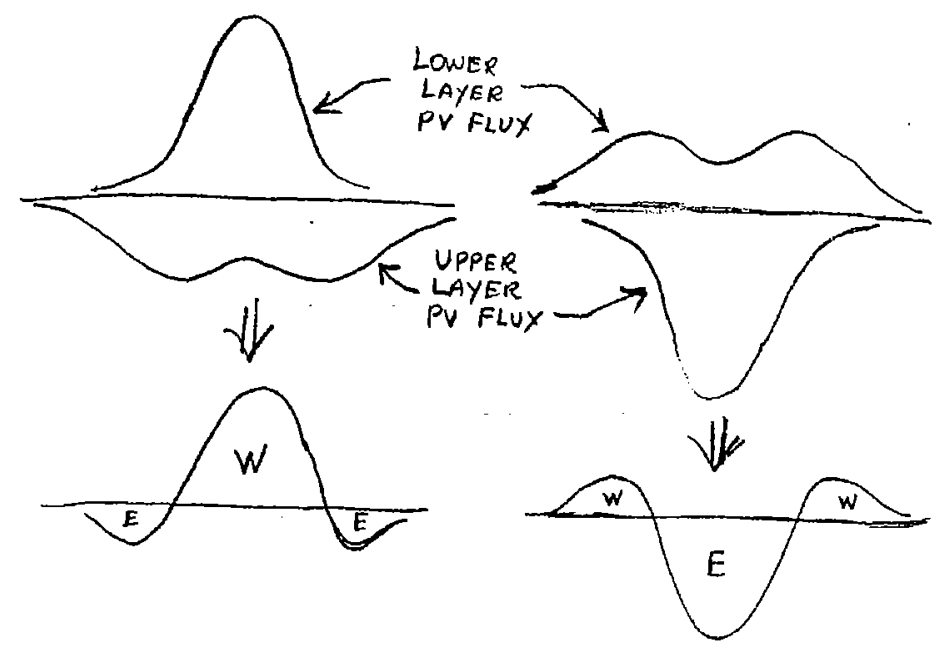

Figure 7: Schematic of two-layer potential vorticity flux distributions that would result in surface westerlies and surface easterlies underneath the region of baroclinic eddy production.

We now need a physical explanation for why we expect to see a broader flux in the upper layer. We expect that the eddies will form and then emanate from the source region until they become nonlinear, break, and mix potential vorticity. Heuristically, we would like to argue that eddies will be more linear in the upper layer, and thereby propagate more readily away from the source region at upper levels. Surface friction is part of the story, but I do not think that it is the dominant part. As a measure of linearity, let us again use the quantity $u^{\prime} /(\bar{u}-c)$. When this quantity becomes large we expect wave breaking and mixing. Baroclinic instability theory (not discussed here) suggests that when $\beta$ is small and the vertical shear and stratification are uniform, the steering level of the unstable waves (the height at which $\bar{u}=c$ ), is in the midtroposphere. However, the $\beta$-effect naturally encourages westward propagation, moving the steering level to lower levels. This implies that $\bar{u}-c$ is larger in the upper troposphere than in the lower troposphere, on average, so that the upper layer supports linear waves better than does the lower layer. Thus, the linear waves are able to propagate further away from the source region and the potential vorticity fluxes occur over a broader latitude band.

A positive feedback results from the generation of surface westerlies, since the presence of these westerlies itself causes $\bar{u}-c$ to decrease at the center of the storm track in the lower 
layer and to increase in the upper layer, accentuating this asymmetry in the nonlinearity. The low level component of a developing baroclinic wave breaks, or "occludes", in the source region, without propagating meridionally appreciably; upper level disturbances do disperse to some extent. Surface friction contributes to this asymmetry as well. We see momentum fluxes in the upper troposphere but relatively little in the lower troposphere. It is this asymmetry that motivates the stirred upper tropospheric model of the first few lectures.

Figure 8 shows the upper level potential vorticity in an idealized two-layer model of an baroclinically unstable jet on a $\beta$-plane. There is relatively little breaking at the jet core, marked by the concentrated red potential vorticity contours near the center of the channel, as compared to the breaking, or rolling up of potential vorticity, seen on both flanks. Indeed, it is this breaking and homogenization on each side of the jet that creates the sharp potential vorticity boundary between them, sharpening the jet. A similar figure at low levels shows maximum mixing at the center of the channel.

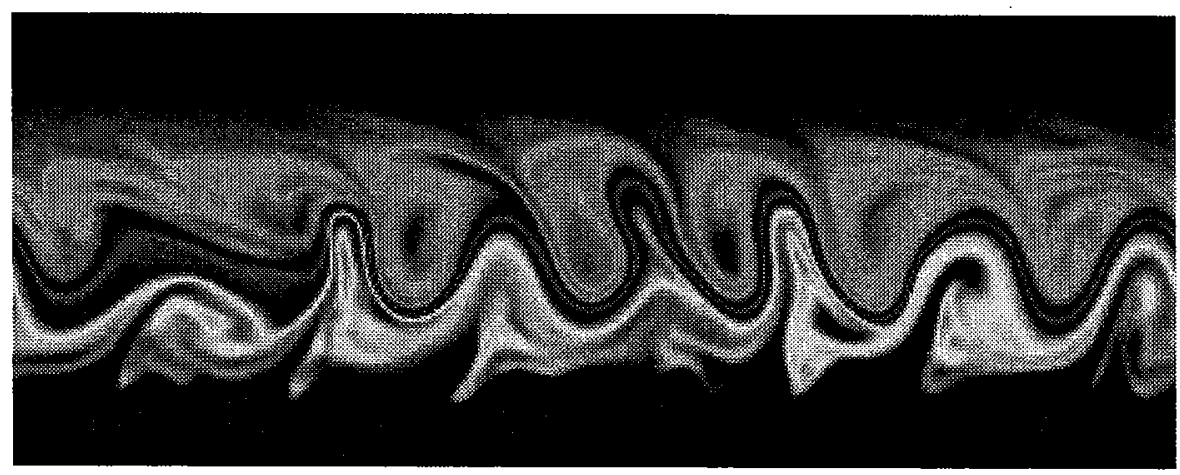

Figure 8: A baroclinically unstable jet

(Without spherical geometry it is difficult for baroclinic waves to propagate significantly away from their source region. On the sphere, as one moves equatorward, the Coriolis parameter in the thermal wind equation gets smaller and one enters a region of very weak temperature gradients and weak baroclinic eddy production even though the upper level winds are still strong enough to support wave propagation. In a QG model on a $\beta$-plane, if one concentrates the vertical shear in a jet, the upper level flow is not strong enough away from the jet to support significant propagation. So Figure 8 would be modified signficantly on the sphere, with the breaking on the equatorward side extended more broadly.)

We can think of eddy production and decay in midlatitudes as a two-step process. In the first step eddies grow baroclinically, reducing the vertical shear, decelerating the upper layer and accelerating the surface westerlies through form drag. In the second step, the upper level disturbance disperses meridionally, eventually breaking and mixing potential vorticity in a broad region, while the lower level eddy field occludes and dissipates. The upper layer deceleration is thereby compensated somewhat, as the dispersion of eddies, primarily into the tropics, redistributing the upper level deceleration towards the tropics.

The critical thickness gradient needed to allow baroclinic instability in this two-layer 
model is determined by setting the lower layer potential vorticity gradient to zero.

$$
0 \sim \frac{1}{q} \frac{\partial q}{\partial y} \sim \frac{1}{f} \frac{\partial f}{\partial y}-\frac{1}{H} \frac{\partial H}{\partial y}
$$

Since

$$
\frac{1}{f} \frac{\partial f}{\partial y} \sim \frac{\bar{\beta}}{f} \sim \frac{\cot \theta}{a}
$$

in midlatitudes the critical gradient is

$$
\left.\frac{\partial H}{\partial y}\right|_{c} \sim \frac{H}{a}
$$

The critical interface slope in this two-layer model is roughly that needed to carry one from the bottom of the model in the tropics to the top of the model near the poles. This is why we never use two-layer models when studying the entire sphere simultaneously. If the flow is baroclinically unstable in midlatitudes, the lower layer will awkwardly disappear in low latitudes, and the upper layer in high latitudes, leaving no vertical resolution over much of the globe. The two-layer model is only useful when isolating mid-latitude, or tropical, dynamics. (Two-level models are very common, but these are not models of a physically realizable layered system; they are just a crude finite differencing of the continuous equations.)

Suppose that the radiative drive is weak. Then we might expect to see an isentropic slope in the final statistically steady state that is close to this critical slope. This picture is often referred to as baroclinic adjustment. This seems like an attractive simple model for the observed isentropic slope in the troposphere. Among the difficulties with this picture is the simple fact that the atmosphere is not a two-layer system but has, instead, continuous stratification. Baroclinic instability theory tells us that the critical shear for instability, in inviscid flow, disappears in the simplest continuously stratified models. (One can think of the number of layers increasing and the thickness of the lowest layer decreasing, so that the isentropic slope required to produce the same fractional thickness gradient decreases.) Two-layer baroclinic adjustment is enticing, but unsatisfying.

\section{Continuous stratification}

We still have a problem explaining the mean overturning circulation in midlatitudes in the continuous limit.

Potential vorticity gradients are generally positive everywhere in the troposphere, because the $\beta$-effect dominates. Therefore, baroclinic instability is not generated by a reversal of the potential vorticity gradient in the most obvious way. As Charney showed, in a continuously stratified atmosphere the baroclinic instability arises because of the existence of a temperature gradient at the surface, with temperatures decreasing polewards. This surface temperature gradient plays the role of the lower layer in our previous model while the entire atmosphere acts as the upper layer! While the mathematics of quasi-geostrophic theory points to this interpretation, its physical implications are not that easily appreciated. Most important is the need to understand the implications of this picture for the overturning circulation in midlatitudes. 
In the upper layer of the two-layer model, mixing results in an equatorward potential vorticity flux and a poleward mass flux. Why can't we apply this same argument to any infinitesimal isentropic layer within the troposphere, which would require a poleward flux in every such layer? Where is the return flow? Quasigeostrophic theory indicates that the return flow is confined to a $\delta$-function at the surface(!). We need to develop a physical understanding of this theoretical construct.

When isentropic layers intersect the surface, the thickness of the layer goes to zero and we have some difficulty in defining potential vorticity. But as long as we are examining a layer that is safely removed from the surface despite being buffeted by baroclinic eddies, we have no such difficulty. In uninterrupted layers, potential vorticity is well defined and, we assume, increases poleward within the layer in the mean. Mixing then produces an equatorward potential vorticity flux and a poleward mass flux. It seems that we have to look to the interrupted layers for the equatorward mass flux. This idea is supported by Figure 7, where one sees that much of the return flow occurs within isentropic layers that are close to, and to a large extent, colder than, the mean surface temperature. Nearly all of this flux is confined to interrupted layers, as discussed in [6]. The return flow can be thought of as occurring in cold air outbreaks near the surface.

Consider the atmosphere at a particular latitude. Divide the atmosphere into two parts: isentropic layers that sometimes intersect the surface at that latitude, and isentropic layers that never (or hardly ever) intersect the surface. We can estimate the total depth of the interrupted layers, $h_{I}$ as roughly

$$
h_{I} \approx \frac{\Theta^{\prime}}{\partial \bar{\Theta} / \partial p}
$$

An estimate of the RMS of $\Theta^{\prime}$ in midlatitudes is $10^{\circ} \mathrm{K}$, and $\partial \bar{\Theta} / \partial p$ is about $3^{\circ} \mathrm{K} / \mathrm{km}$. Thus, the interrupted layer extends through about one-third of the troposphere. In quasigeostrophic theory this thickness is assumed to be infinitesimal! (It is assumed that static stability variations are small compared to the mean static stability, but the former are assumed to scale as the temperature perturbation divided by the total depth of the troposphere, $H$, which implies that $h_{I}<<H$ ).

Assume that surface temperature is distributed symmetrically about the mean, as seems in fact to be a fairly good approximation in the atmosphere. The probability distribution $P\left(\Theta_{s}\right)$ is normalized so that

$$
\int_{0}^{\infty} P\left(\Theta_{s}\right) d \Theta_{s}=1 .
$$

Assume, in addition, so as to provide a very simple way of thinking about mass fluxes in the interrupted layers, that the surface potential temperature is perfectly correlated with the meridional velocity throughout these layers:

$$
v^{\prime}=\Theta_{s}^{\prime} \alpha
$$

This actually holds fairly well in our atmosphere - when the surface wind is from the south the air is warmer than average, a consequence of the fact that surface temperatures are very strongly forced and the mixing induced by baroclinic waves is not able to deform this distribution profoundly. 
Examine the mass flux in an infinitesimally thin layer of potential temperature $\Theta$. Assume that the static stability is uniform, so that whenever the layer exists (i.e. $\Theta>\Theta_{s}$ ), it has a constant thickness $H$. Thus, the instantaneous mass flux through the layer is $H v^{\prime}$. However, we also need to take into account how often the layer is present, so we weight the instantaneous mass flux by the probability density. The average mass flux $M$ through the layer is

$$
M(\Theta)=H \int_{0}^{\Theta} P\left(\Theta_{s}\right) v^{\prime} d \Theta_{s} .=H \alpha \int_{0}^{\Theta}\left(\Theta_{s}-\bar{\Theta}_{s}\right) P\left(\Theta_{s}\right) d \Theta_{s}
$$

From this expression, we see that this mass flux decays to zero when either the surface temperature is much colder or much warmer than the mean. When the temperature is too warm, the layer is almost always present, but both poleward and equatorward flows occur and cancel, resulting in very little net mass flux. When the temperature is too cold, the layer is never present so, once again, there is no flux. The maximum mass flux is expected to occur in layers with temperatures that are close to the mean surface temperature. In fact, if the probability distribution is a Gaussian, and accepting the perfect correlation between perturbation temperature and meridional wind, the distribution of the mass flux will also be a Gaussian, centered at the mean surface temperature. Half of the mass transport will occur in layers that are colder than the mean.

We have neglected the planetary boundary layer (PBL) in determining the surface layer mass transport. This is a problem because the PBL is well-mixed, so there is no vertical entropy gradient. To account for the $\mathrm{PBL}$, we need to recognize first that the argument above applies to the interrupted layers above the PBL, and that the static stability referred to above is the static stability in the free troposphere. If we assume that the PBL is always present, and if its thickness is not correlated with surface temperature, then there will be no net mass flux in the layer, although it will contribute poleward transport to those isentropic layers that are above the mean and equatorward transport to those below the mean. This will simply have the effect of shifting the distribution of the return flow to colder temperatures. (See [6] to read more about this confusing point.) Additional effects could arise due to correlations between the thickness of the PBL and the surface temperature, however.

Potential temperature is conserved along the surface in the absence of diabatic forcing, and midlatitude eddies try to mix the potential temperature (although they are far from successful in homogenizing it) causing a downgradient flux in the usual way. If we assume that the meridional flow has little vertical structure throughout this layer and that the mean static stability is uniform (both of these assumptions are motivated by the quasi-geostrophic scaling limit) then the mass flux is simply

$$
\frac{1}{g} \frac{\overline{v^{\prime}\left(\Theta_{I}-\Theta_{s}^{\prime}\right)}}{\partial \bar{\Theta} / \partial p}=-\frac{1}{g} \frac{\overline{v^{\prime} \Theta_{s}^{\prime}}}{\partial \bar{\Theta} / \partial p}
$$

Here $\Theta_{I}$ is a constant that marks the top of the interrupted layer at the latitude of interest. The mass flux in this layer is equal, but opposite in sign, to the surface heat flux divided by the static stability above the planetary boundary near the surface. The interrupted layers play the role of the lower layer in the two-layer model, with the irreversible mixing 
of potential temperature along the surface generating an equatorward flow balancing the poleward flow forced by potential vorticity mixing in the uninterrupted interior layers.

The Coriolis force on this mass transport balances the form drag at the top of this layer. There is, in addition, mass transport in the surface layers needed to balance the surface stresses. The former is due to geostrophic eddies, the latter to an ageostrophic Ekman drift.

\section{The Hadley cell}

Return now to the budget of angular momentum $M=(\Omega a \cos \theta+u) a \cos \theta$ in the latitudepressure plane. In pressure coordinates $(\omega \equiv D p / D t)$ :

$$
\frac{\partial \bar{M}}{\partial t}=-\frac{1}{a \cos \theta} \frac{\partial(\cos \hat{\theta v M})}{\partial \theta}-\frac{\partial(\overline{\omega M})}{\partial p}
$$

or

$$
\begin{aligned}
a \cos \theta \frac{\partial \bar{u}}{\partial t} & =-\frac{1}{a \cos \theta} \frac{\partial(\cos \theta \bar{v} \bar{M})}{\partial \theta}-\frac{\partial(\bar{\omega} \bar{M})}{\partial p} \\
& -\frac{1}{a \cos \theta} \frac{\partial\left(\cos \theta \overline{v^{\prime} M^{\prime}}\right)}{\partial \theta}-\frac{\partial\left(\omega^{\prime} M^{\prime}\right)}{\partial p}
\end{aligned}
$$

A bit of algebra, utilizing the relation between the mean absolute vorticity and meridional gradient of the mean angular momentum,

$$
f+\bar{\zeta}=-\frac{1}{a^{2} \cos (\theta)} \frac{\partial \bar{M}}{\partial \theta}
$$

yields

$$
\frac{\partial \bar{u}}{\partial t}=(f+\bar{\zeta}) \bar{v}-\bar{\omega} \frac{\partial \bar{u}}{\partial p}-\frac{1}{a \cos ^{2} \theta} \frac{\partial\left(\cos ^{2} \theta \overline{u^{\prime} v^{\prime}}\right)}{\partial \theta}-\frac{\partial \overline{\omega^{\prime} u^{\prime}}}{\partial p}
$$

For the purpose of developing a qualitative picture of the circulation, we can think of the final term on the RHS, the vertical eddy flux of zonal momentum, as only important in the planetary boundary layer.

One could, at this point, perform analogous manipulations to those used in our discussion of the two-layer quasi-geostrophic model, to express the mean flow modification in terms of the residual circulation. But we now want to discuss the low latitude circulation, and eddy thickness fluxes and the associated form drag drop to very small values rather quickly as one moves equatorward of the subtropical jet. The potential vorticity flux is dominated by the momentum flux convergence, and the residual circulation and the Eulerian mean circulation are nearly the same. So we will not bother with this reorganization here.

If we also ignore the transport of relative angular momentum by the mean meridional circulation, we have, outside of the boundary layer,

$$
\frac{\partial \bar{u}}{\partial t}=0=f \bar{v}-\frac{1}{a \cos ^{2} \theta} \frac{\partial\left(\cos ^{2} \theta \overline{u^{\prime} v^{\prime}}\right)}{\partial \theta}
$$

which is the expression that formed the basis of our discussion of the momentum balance in midlatitudes. 
The low Rossby number assumption $(\zeta<<f)$ breaks down as one moves to lower latitudes. This results in a fundamental change in the way in which the overturning circulation in the troposphere is controlled. In the low Rossby number limit the overturning circulation is a slave to the eddy stresses in a steady state, as the Coriolis force on the meridional circulation must balance the stress. In low latitudes, one intuitively expects that the thermal forcing must exert a direct effect on the overturning. Since the eddy stresses are due to potential vorticity mixing associated with disturbances generated in midlatitudes and spreading into the tropics, the connection between these stresses and tropical forcing is subtle at best, so it is difficult to see how this effect can be mediated through changes in these eddy stresses. To clarify this point, it is useful to simplify the problem by dropping the vertical advection of momentum by the mean circulation and retaining only the horizontal advection:

$$
0=\frac{\partial \bar{u}}{\partial t}=(f+\bar{\zeta}) \bar{v}-S
$$

where $S$ is the eddy stress. While not obviously justifiable by a scale analysis, it turns out that the vertical advection term would modify our arguments in only a modest way. (It just so happens that the strongest vertical mean flows in the tropics occur where the vertical shear is weak. If we consider "superrotating" atmospheres, in which there is strong vertical shear in the deep tropics, then this term does become important - see [19].)

The overturning is no longer tied to the eddy stresses. If the thermal forcing in the tropics is increased, for example, one can imagine that $\bar{v}$ will increase, even if the eddy stresses in the upper troposphere are not changed. One can still satisfy the momentum balance if the absolute vorticity is reduced in the upper troposphere. Since the absolute vorticity is just the gradient of angular momentum, one is simply saying that, in the presence of a stronger circulation, the same stresses do not have as much time to extract zonal momentum from the poleward moving air.

In the extreme case in which the eddy stresses are reduced to zero, the mean meridional circulation has two options. Either $\bar{v}$ vanishes (the only possibility in the low Rossby number limit), or $f+\bar{\zeta}$ vanishes. In the latter case, a mean flow still exists, that conserves its angular momentum as it moves polewards. The circulation must then clearly be determined by the thermal forcing.

This is, in a nutshell, the key dynamical distinction between the tropical and midlatitude general circulations. In the tropics, the overturning circulation can and does respond to changes in thermal forcing, independently of changes in eddy stress. In midlatitudes, the overturning cannot be altered significantly without altering the eddy stresses. What determines the size of the "tropics" from this perspective?

Consider the upper layer of a two layer model, assuming homogeneous incompressible layers for simplicity. The zonal flow in the lower layer is assumed to be negligible due to surface friction. The flow is driven by "radiative forcing" that produces mass fluxes between the two layers which relax the interface towards some radiative equilibrium shape. The pressure at the upper boundary is assumed to be constant. The steady-state equations for axisymmetric flow in the resulting reduced-gravity shallow water model are (dropping 
the overbars)

$$
\begin{aligned}
& 0=\frac{\partial u}{\partial t}=(f+\zeta) v-S \\
& 0=\frac{\partial v}{\partial t}=-f u-\frac{g^{\star}}{a} \frac{\partial H}{\partial \theta}-\frac{v}{a} \frac{\partial v}{\partial \theta}-u^{2} \frac{\tan (\theta)}{a} \\
& 0=\frac{\partial H}{\partial t}=-\frac{1}{a \cos (\theta)} \frac{\partial}{\partial \theta}(\cos (\theta) v H)-\frac{H-H_{e q}(\theta)}{\tau}
\end{aligned}
$$

where $H$ is the thickness of the upper layer, $H_{e q}(\theta)$ the radiative equilibrium thickness, $\tau$ the radiative relaxation time, and $g^{\star}$ the reduced gravity. We have ignored the vertical momentum transfer associated with the mass transfer between layers, equivalent to neglecting the vertical advection of momentum in the discussion above. (Again, this is adequate for circulations similar to that existing on Earth but not for flows with strong vertical shear at the equator.) For the parameter range we are interested in the meridional flow is much weaker than the zonal flow, and we can ignore the meridional advection of the meridional flow itself in the $v$-equation. (One can check after the fact that our solutions would be modified by this term only in a very small region around the equator extending to only $1-2^{\circ}$ latitude.) This would leave us with gradient wind balance, but neglecting the term proportional to $u^{2}$ is also justified as long as the zonal flows are weak compared to $\Omega a$. The meridional equation of motion is then simply geostrophic balance.

$$
0=\frac{\partial v}{\partial t}=-f u-\frac{g^{\star}}{a} \frac{\partial H}{\partial \theta}
$$

We still need to specify $S$. Let's assume that $S=\kappa s(\theta)$, with $s>0$ everywhere except at the equator where $s=0 . \kappa$ is a parameter that allows us to pass to the inviscid limit. We will be considering solutions that are forced symmetrically about the equator, so that $v=0$ at the equator. If $u=0$ at the equator initially, this will be true for all times, and we will only look for solutions of this type. If $S$ does not vanish at the equator, this model could not reach a steady state, since we have neglected the vertical momentum exchange that would be required in this model to balance any imposed stresses at the equator! As an additional simplification, we can linearize the thickness equation about the mean thickness, $H_{0}$. The only important nonlinearity in this model is in the zonal momentum equation.

The maximum value of angular momentum must be attained at the equator in this model. Suppose this were not the case, and there were a point off the equator at which $M$ were a local maximum. The absolute vorticity would vanish at this point, but there would be nothing balancing non-zero eddy stresses. Therefore, we must have $u \leq u_{M}(\theta)$, where $u_{M}$ is the wind field obtained by conserving angular momentum, stating with $u=0$ at the equator.

When $\kappa=0$, one solution of these equation is simply radiative equilibrium: $H=$ $H_{e q} ; u=u_{e q}, v=0$. But this cannot be the limit of the solutions for non-zero $\kappa$ as $\kappa \rightarrow 0$, since $u_{e q}$ does not vanish at the equator. This is a special case of a centrally important fact: radiative equilibrium temperatures cannot, typically, be realized close to the equator, for the temperature gradients would force unphysical upper tropospheric winds. This is even more self-evident if we allow radiative equilibrium to have a meridional gradient at the equator, for then the radiative equilibrium winds are infinite! 
Let's assume the equilibrium height field is parabolic close to the equator:

$$
H_{e q} \approx H_{o}\left(1-\alpha \theta^{2}\right)
$$

Then,

$$
f u_{e q}=-\frac{g^{*}}{a} \frac{\partial H_{e q}}{\partial \theta} \approx \frac{2 \alpha g^{*} H_{o}}{a} \theta
$$

Since $f=2 \Omega \theta$ near the equator, we have

$$
\frac{u_{e q}}{\Omega a} \approx \alpha R \Delta_{V}
$$

where

$$
R=\frac{g H_{o}}{\Omega^{2} a^{2}}
$$

We have set $g^{*}=g \Delta_{V}$, where $\Delta_{V}$ is the fractional change in potential temperature in the vertical.

This expression gives the misleading impression that these radiative equilibrium winds depend on the static stability of the atmosphere. To see why this is not the case, assume, for example, that the radiative-equilibrium potential temperature field is of the form

$$
\frac{\Theta_{e q}}{\Theta_{0}}=1-\Delta_{H} \sin ^{2}(\theta)+\Delta_{V} \frac{z}{H} \approx 1-\Delta_{H} \theta^{2}+\Delta_{V} \frac{z}{H}
$$

Think of the bottom of the upper layer as a surface of constant $\Theta$. Then the thickness of the upper layer of our two-layer model, in radiative equilibrium, would be

$$
\frac{H_{e q}-H_{0}}{H_{0}} \approx 1-\frac{\Delta_{H}}{\Delta_{V}} \theta^{2}
$$

Therefore, $\alpha \approx \Delta_{H} / \Delta_{V}$, and we find

$$
\frac{u_{e q}}{\Omega a} \approx R \Delta_{H}
$$

It is the horizontal temperature gradients in radiative equilibrium, not the static stability, that enters this expression. For the Earth, a relevant value might be $\Delta_{H} \approx 0.3$ (implying a pole-to-equator temperature difference in radiative equilibrium of $100 \mathrm{~K}$ ) and $R \approx 0.7$ so that $R \Delta_{H} \approx 0.2$

Whatever the value of $\kappa$, a circulation must exist to cause the flow to depart from this radiative equilibrium throughout the region in which $u_{e q}>u_{M}$. Continuing to make the small angle approximation,

$$
u_{M} \approx \Omega a \theta^{2} \text {. }
$$

a circulation must exist at least from the equator up to the latitude

$$
\theta_{H} \approx \sqrt{R \Delta_{H}}
$$

In the limit $\kappa \rightarrow 0$, since $v \neq 0$ we must have zero absolute vorticity, or $u=u_{M}$ in this region. 
To be more precise, assume that as $\kappa \rightarrow 0$ there is a latitude $\theta_{H}$ equatorward of which $v \neq 0$ and $u=u_{M}$, and poleward of which $v=0$ and $u=u_{e q}$. We refer to the region $\theta<\theta_{H}$ as the "tropics". Within the tropics, we can compute the thickness gradient consistent with angular momentum conservation:

$$
\frac{g^{\star}}{a} \frac{\partial H}{\partial \theta} \approx-2 \Omega^{2} a \theta^{3}
$$

Integrating from the equator, we obtain the thickness distribution

$$
\frac{H(\theta)-H(0)}{H_{0}}=-\frac{1}{2 R \Delta_{H}} \theta^{4}
$$

This height field is extremely flat in the deep tropics, so as to keep the upper tropospheric winds from increasing faster than allowed by angular momentum conservation, or, equivalently, by the criterion of inertial stability. (A balanced flow with angular momentum increasing as one moves towards the axis of rotation - i.e., polewards on the sphere - is inertially unstable.) Keep in mind that this is an upper limit on the height gradients in the tropics; significant eddy stresses would reduce this gradient even further.

We need two constraints to allow us to solve for $\theta_{H}$ and $H(0)$ simultaneously. The constraints are continuity of $H$ at $\theta=\theta_{H}$ and mass conservation, which requires that

$$
\int_{0}^{\theta_{H}}\left(H(\theta)-H_{e q}\right) d \theta=0
$$

The "equal-area" construction in Figure 9 allows one to picture how these two constraints are satisfied simultaneously. One sees from the figure that the height gradient at the boundary of the tropics will be discontinuous, and that the zonal wind overshoots the radiative equilibrium value. In the small angle approximation, the result of this construction is $\theta_{H}=\sqrt{\frac{5}{3} R \Delta_{H}}$. For $\Delta_{H} R \approx 0.2$ one gets $\theta_{H} \approx 0.6 \approx 30^{\circ}$. The small angle approximation is evidently only marginally adequate for these parameters, but calculations on the sphere do not change things much. See [4].

Figure 10 shows numerical solutions to this set of equations on the sphere, in which $H_{e q}=H_{e q}(0)-\alpha \sin ^{2}(\theta)$ and with $S=-\kappa u$. This form for $S$ is not meant to capture any of the physics of the actual drag in the upper troposphere; it is just a simple way of illustrating the essential distinction between the tropical and extratropical balances in the model.

This simple model of the Hadley cell is easy to criticize as unrealistic. For one thing, if an angular momentum conserving flow extended to the subtropics, winds in excess of $130 \mathrm{~m} / \mathrm{s}$ would be generated. (Most of these strong winds are generated after one moves past $\approx 20^{\circ}$ ) The observed zonally averaged subtropical jet is less than $40 \mathrm{~m} / \mathrm{s}$. Obviously, eddy stresses are substantial once the flow approaches the subtropics. There are a number of open questions related to modeling the eddy stresses in a more physically relevant way and then studying how these stresses interact with the Hadley cell. One starting point might be linear "Rossby wave chromatography", in which a a phase speed spectrum of the input of pseudomentum in midlatitudes is prescribed, and then each wave deposits its drag at its critical latitude. However, this cannot be appropriate when the model is close to its 


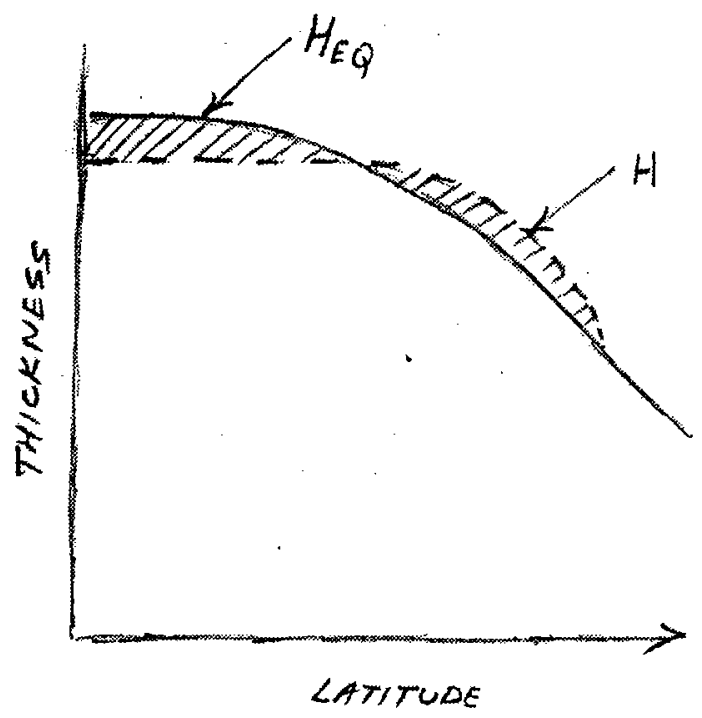

Figure 9: Simultaneously satisfying continuity of temperature at the subtropical boundary of the Hadley cell and mass conservation
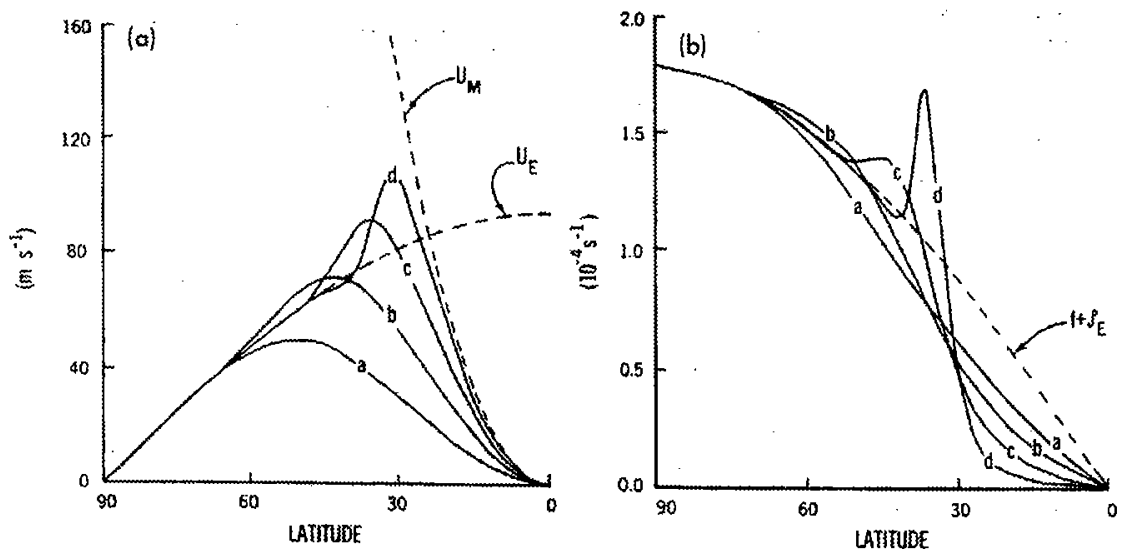

Figure 10: Solutions to the idealized Hadley cell model as the eddy stresses in the zonal momentum equation are reduced in strength. The zonal winds and the absolute vorticity are shown. 
nearly inviscid limit, in which there is little vorticity gradient in the tropics, so that Rossby waves are not able to propagate past the subtropical jet to get to their critical latitude. This suggests an interesting "bootstrap" feedback in which the eddy stresses themselves create the vorticity gradient that supports their propagation into the tropics.

An alternative, and more traditional, view of how the width of the Hadley cell is determined is that angular-momentum conservation continues polewards until the resulting vertical shears become baroclinically unstable. Suppose we use the two-layer model's criterion for instability for this purpose:

$$
\beta-\frac{f}{H_{0}} \frac{\partial H}{\partial y}=0 \Rightarrow \frac{\partial H}{\partial y}=\frac{\beta H_{0}}{f} .
$$

From thermal wind balance,

$$
g^{*} \frac{\partial H}{\partial y}=f\left(u_{1}-u_{2}\right)
$$

With $u_{2} \approx 0$, then, in the upper troposphere

$$
u_{1} \approx \beta \lambda^{2}=\beta \frac{g^{*} H_{0}}{f^{2}}
$$

or

$$
\frac{u}{\Omega a} \approx R \Delta_{V} \frac{\cos (\theta)}{\sin ^{2}(\theta)}
$$

On the other hand, for the angular momentum conserving wind we have:

$$
\frac{u_{M}}{\Omega a} \approx \frac{\sin ^{2}(\theta)}{\cos (\theta)}
$$

Invoking the small angle approximation for algebraic simplicity once again. we solve the equation $u_{1}=u_{M}$ to obtain the latitude, $\theta_{B C}$, at which the two wind profiles coincide, in the small angle approximation,

$$
\theta_{B C} \approx\left(R \Delta_{V}\right)^{\frac{1}{4}} \text {. }
$$

The relationship between the axisymmetric theory and this instability argument is

$$
\theta_{B C}^{4}=\frac{\Delta_{V}}{\Delta_{H}} \theta_{H}^{2}
$$

One could combine the two theories by saying that the Hadley cell stops at the smaller of $\theta_{B C}$ and $\theta_{H}$. If $\theta_{B C}<\theta_{H}$, the flow would become unstable before reaching the axisymmetric limit. If $\theta_{H}>\theta_{B C}$, on the other hand, the Hadley cell would terminate before becoming unstable. Is the latter situation physically realizable? In an atmosphere heated from below, what would maintain the static stability in the region between the edge of the Hadley cell and the start of the baroclinically unstable zone? Would the isentropic slope, $\Delta_{V} / \Delta_{H}$, simply adjust so as to remove this gap?

One could use some other instability criterion in this argument, rather than rely on the existence of a critical shear in the two layer model, the physical relevance of which is obscure 
at best. For example, an estimate of growth rate $\omega_{I}$ from the Eady or Charney models is $f R i^{-1 / 2} \approx f u / N H$, or

$$
\omega_{I} \approx \frac{f u}{\sqrt{g H \Delta_{V}}} \approx 2 \frac{u}{a \sqrt{R \Delta_{V}}} \theta
$$

If we suppose that this growth rate must be larger than some frictional damping $\kappa$, due to boundary layer drag, for example, then the angular momentum conserving wind would reach this value at

$$
\theta_{\kappa}^{3} \approx \frac{\kappa}{2 \Omega} \sqrt{R \Delta_{V}}
$$

Even better would be to use the kind of diffusive closure theory described below, and ask where the flux divergence due to the resulting heat flux becomes comparable to the radiative forcing. The details are different, but the qualitative behavior is the same.

\section{A Moist Hadley Cell}

People are often amazed that one would try to talk about the Hadley cell without talking about moist convection, as in the previous section. How does moist convection change this picture? Although less often remarked upon, it should also seem strange that one would talk about the Hadley cell without taking into account the oceanic circulation in the tropics, which transports more energy polewards than does the atmospheric circulation. These two issues are related.

To approach this subject we look first at the energy balance at the top of the atmosphere. For our purposes, we can think of the stratosphere as in radiative equilibrium, so we are really thinking about the energy fluxes at the tropopause. Let's also start by ignoring any energy transport by the ocean so that, in a steady state, there are no fluxes through the surface, and the energy fluxes at the tropopause are the only sources of energy for the troposphere.

The incoming solar radiation $S(\theta)$ has to balance the outgoing infrared radiation $I(T)$. We assume that $S$ is only a function of latitude and $I$ only a function of $T$. These assumption obviously ignore all sorts of complexity associated with the effects of clouds and water vapor on these radiative fluxes. Integrated over the Hadley cell, and ignoring any energy fluxes out of the Hadley cell into midlatitudes, we require

$$
\int_{0}^{\theta_{H}}(S(\theta)-I(T)) \cos (\theta) d \theta=0
$$

Assuming once again that the zonal mean flow is in thermal wind balance,

$$
f \frac{\partial u}{\partial z}=-\frac{g}{T} \frac{\partial T}{\partial y}
$$

and integrating this equation from the surface up to the tropopause, assuming that $u(0) \approx 0$ we get

$$
u(H)=-\frac{g H}{f T_{0}} \frac{\partial[T]}{\partial y}
$$

where $[T]$ is the vertically averaged temperature. We are also assuming that the height of the tropopause is independent of latitude. We assume that we can think of $I$ as a function 
of this vertically averaged temperature, $[T]$, i.e., that variations of the temperature field are always sufficiently coherent in the vertical within the tropics. We drop the brackets in the notation for this vertically averaged temperature from here on.

The Hadley cell theory can now proceed exactly as before. Angular momentum conservation implies a temperature profile $T(\theta)-T(0)$ and a profile of outgoing radiation. Continuity of $T$ at the boundary of the cell and conservation of energy result in the same equal area construction for $T(0)$ and the width of the cell. The solution includes the energy transport by the circulation. It does not tell us its strength, that is, the mass transport. To obtain the mass transport, we need additional information. It is the ratio of energy to mass transport that is affected by the presence of water vapor.

We need to digress a bit to talk about energy conservation in a compressible fluid. Start with the conservation of kinetic and potential energy,

$$
\rho \frac{D}{D t} \frac{1}{2}|\mathbf{v}|^{2}=-\rho \mathbf{v} \cdot \mathbf{v} \Phi-\mathbf{v} \cdot \nabla p
$$

or

$$
\rho \frac{D}{D t}\left(\frac{1}{2}|\mathbf{v}|^{2}+\Phi\right)=-\nabla \cdot(p \mathbf{v})+p \nabla \cdot \mathbf{v}
$$

Similarity for internal energy,

$$
\rho \frac{D e}{D t}=Q-p \nabla \cdot \mathbf{v}
$$

Here $\Phi=g z$ is the geopotential and $Q$ is the heating rate. Adding the kinetic, potential, and internal energies, we obtain the expression for energy conservation:

$$
\rho \frac{D}{D t}\left(\frac{1}{2}|\mathbf{v}|^{2}+\Phi+e\right)=-\nabla \cdot(p \mathbf{v})+Q
$$

Using the continuity equation, we may write this in flux form:

$$
\frac{\partial}{\partial t} \rho\left(\frac{1}{2}|\mathbf{v}|^{2}+\phi+e\right)=-\nabla \cdot\left(\rho \mathbf{v}\left(\frac{1}{2}|\mathbf{v}|^{2}+\phi+h\right)\right)+Q
$$

where $h=e+p / \rho$ is the enthalpy. For our ideal gas, $e=c_{v} T$ and $h=c_{p} T$. Note that $e$ appears on the LHS and $h$ on the RHS. We also define the dry static energy $s$

$$
s=\phi+h=c_{p} T+g z
$$

The kinetic energy is a very small part of the total energy in the atmosphere (recall that $\left.c_{p} \approx 10^{3} \mathrm{~m}^{2} /\left(s^{2} K\right)\right)$. so we can compute the energy flux from the flux of dry static energy. In a steady state

$$
\nabla \cdot(\rho \mathbf{v} s)=Q
$$

If the dry stability of the atmosphere is positive, then the dry static energy increases with height:

$$
\frac{\partial s}{\partial z}=c_{p} \frac{\partial T}{\partial z}+g=c_{p} \frac{T}{\Theta} \frac{\partial \Theta}{\partial z} .
$$


The north south flow in the Hadley cell is concentrated near the surface and near the tropopause. We refer to the dry static energy characterizing the surface air as $s(0)$ and that of the air near the troposphere as $s(H)$. Vertically integrating, we have

$$
\nabla \cdot\left(V \Delta_{D}\right)=\int Q
$$

where $V$ is the mass flux in either the poleward or equatorward branch of the cell and $\Delta_{D} \equiv s(H)-S(0)$ is referred to as the gross dry stability of the overturning circulation. It is simply the ratio of the dry static energy transport to the mass transport. In the tropics, $\Delta_{D} \approx\left(45^{\circ} K\right) c_{p}$.

The heating rate $Q$ is comprised of the vertical divergence of radiative and sensible heat fluxes, and latent heat release. Therefore, the vertical integral of $Q$ can be written as the sum of three terms: 1) the difference between the radiative fluxes at the top and bottom of the atmosphere; 2) the sensible heat flux at the surface; and 3) the total latent heating.

$$
\nabla \cdot\left(V \Delta_{D}\right)=R_{T}+R_{B}+S+L P
$$

where $L$ the latent heat of condensation, $P$ the precipitation, and fluxes are positive when directed into the atmosphere. However, the total latent heating is also related to the convergence of vapor in the atmosphere and the evaporation $E$. In a steady state, conservation of water vapor implies that

$$
\nabla \cdot(\rho \mathbf{v} q)=E-P
$$

where $q$ is the mixing ratio for water vapor. We can define a moist static energy, $m \equiv s+L q$, and a gross moist stability, $\Delta_{m}=m(H)-m(0)$, so that

$$
\nabla \cdot\left(V \Delta_{M}\right)=R_{T}+R_{B}+S+L E
$$

But $R_{B}+S+L E=0$ if there is no flux through the surface, so

$$
\nabla \cdot\left(V \Delta_{M}\right)=R_{T}=S(\theta)-I(T)
$$

The energy transport by the Hadley cell is determined by the requirement that it create a temperature distribution that is flat enough to be consistent with upper level winds that are, at most, angular momentum conserving. The mass transport in the cell can then be determined if one has a theory for the gross moist stability (or, conversely, the gross moist stability can be determined if one has a theory for the mass transport!)

The gross moist stability in the tropics is much smaller than the dry stability because nearly all of the water vapor resides at low levels. Note that the energy transport must be smoothly varying in latitude in this theory, since $T$ varies smoothly. The only way to create sharp structure in the mass flux, as at an ITCZ (a region of very concentrated upward motion) is through structure in $\Delta_{M}$, and, in particular, by having $\Delta_{M} \approx 0$ in some region.

In reality, there is a lot of energy flowing from atmosphere to ocean in the tropics, and this energy is removed by transport polewards in the oceans. Indeed there is something we can call the oceanic Hadley cell in the tropics. Much of the circulation in the tropical oceans is wind-driven. The easterlies in low latitudes cause a poleward Ekman drift in the oceanic mixed layer, which results in equatorial upwelling. This circulation is closed by subduction 
of water in the subtropics which returns by complicated routes to supply the equatorial upwelling. Ekman transport is defined as the mass transport required in the boundary layer so that the resulting Coriolis force balances the surface stress. Since the surface stress on the two media is by definition equal and opposite, Ekman mass transports are also equal and opposite in atmosphere and ocean. If we can ignore stresses on land, and if the Ekman transport is the main thing going on in the surface layers of both atmosphere and ocean, then we can expect the mass transport in the oceanic Hadley cell to be comparable to that in the atmospheric Hadley cell (the latter is about 60 Sverdrups in the annual mean). This seems to be the case in the tropics.

We can define an oceanic gross stability, $\Delta_{O}=c_{O} \delta T$, where $c_{O}$ is the heat capacity of water and $\delta T$ the characteristic temperature difference between the poleward and equatorward moving water. Since the oceanic interior is adiabatic, we can assume that $\delta T$ is also the characteristic horizontal temperature difference across the Hadley cell. The atmospheric gross stability is also closely related to this surface temperature gradient; since the atmosphere is nearly saturated at low levels, one can obtain the moisture gradient, which is the key term in the gradient of moist stability, from the temperature gradient.

The ratio of the total energy transport in the system to the mass transport in either ocean or atmosphere is determined by the sum of the gross stabilities of the two media, $\Delta_{t o t} \equiv \Delta_{O}+\Delta_{M}$. In the deep tropics one finds that most of the stability of the system resides in the ocean, so the ocean provides most of the energy transport. It is the energy transport in the atmosphere-ocean system as a whole that is determined by our equal area argument. The contributions of atmosphere and ocean to $\Delta_{t o t}$ determine how this energy transport is partitioned between the two fluids.

\section{Superrotation}

Suppose that there is a local maximum of angular momentum $M$ somewhere in the atmosphere away from the surface. Surround this point by a surface on which the mean $M$ is a constant. By conservation of mass, the mean transport of $M$ into or out of this volume is zero. If there is any downgradient molecular (or small scale turbulent) vertical transport at all, this will remove $M$ from this region; to maintain a steady state we need countergradient eddy fluxes. This does not typically occur in the troposphere, although it does in the stratosphere in the westerly phase of the QBO. where eastward propagating Kelvin and/or gravity waves provide a countergradient vertical flux. In the troposphere, as we have seen, the dominant large scale flux is horizontal and down the angular momentum gradient, from the tropics towards midlatitudes. It is an interesting fact that on Jupiter, Saturn, and the Sun the atmosphere near the equator rotates faster than the planetary interior, as measured by the rotation of the magnetic fields or by helioseismology, so maybe countergradient fluxes are the rule, rather than the exception. Could the upper troposphere superrotate?

Remarkably, some idealized models of the atmosphere do produce an abrupt transition to a superrotating state as one varies certain parameters. Figure 11 shows perhaps the best example [21].

This result is from a dry two-level model on the sphere, forced by linear relaxation to a specified radiative equilibrium temperature distribution. One starts with zonally symmetric forcing and then perturbs the system by modifying radiative equilibrium in the tropics so as 


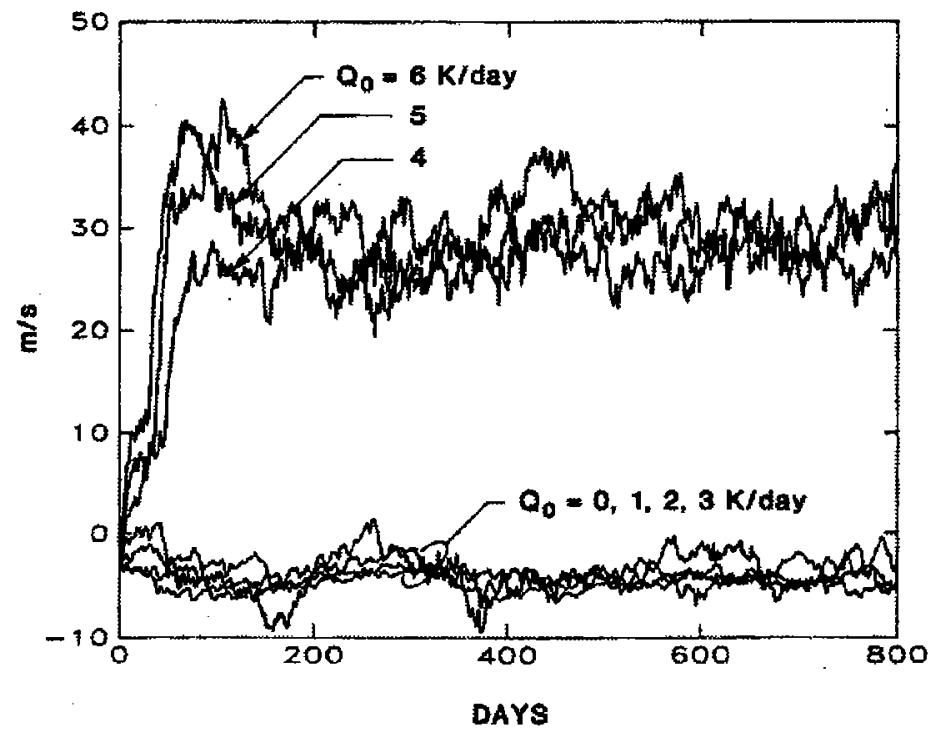

Figure 11: Zonal mean wind at the equator in the upper layer of a two layer model, as a function of time, for different values of the strength of the asymmetric component of tropical heating, from [21]

to be more and more zonally asymmetric. At a certain strength of this asymmetric forcing, the model atmosphere makes a transition from a perfectly normal circulation, with near zero equatorial flow and strong subtropical jet streams, to one with very strong equatorial superrotation, with an upper tropospheric wind field that is closer to solid body rotation.

The starting point for understanding this behavior is that the asymmetric tropical forcing excites a stationary disturbance that will propagate out of the tropics in the upper troposphere, to the extent that conditions are favorable for the existence of Rossby waves. Waves emanating from the tropics are presumably dissipated in various complex ways once they enter midlatitudes. So there is a source of Rossby wave pseudomomentum in the tropics and a sink in midlatitudes. The result, as we have seen, will be a convergence of eddy angular momentum flux and acceleration of the tropical flow. But why should there is such an abrupt transition from the normal to the superrotating state?

There is a fascinating feedback between the strength of the westerlies in the tropical upper troposphere and the propagation and dissipation of the waves excited in midlatitudes by baroclinic instability. We have seen that disturbances excited in midlatitudes will propagate into the tropics, the extent of this propagation depending on how much wave breaking occurs along the way. Wave breaking, in turn, depend in part on the ratio of the wave's zonal wind perturbations as compared to the mean wind in the reference frame moving with the wave's phase speed. The dominant phase speed $c$ of these waves is of the order of $5-12 \mathrm{~m} / \mathrm{s}$. In the normal state, $\bar{u}$ decreases to 0 as one approaches the equator, so breaking is inevitable before one reaches into the deep tropics. But if one can accelerate the tropical upper level winds, breaking will be less likely to occur. This is a positive feedback on the tropical winds because one is thereby losing the deceleration associated with the 
breaking. Eventually, once the equatorial winds are strong enough, the tropics evidently becomes rather transparent to this Rossby wave activity, with little breaking occurring there. This loss of wave breaking and deceleration is strongest as the mean flow rises above the dominant phase speeds in the wave field, and this seems to be the primary cause of the bifurcation in this model.

The problem is actually quite a bit more complicated than this, however. There are at least two other potential feedback mechanisms. First, the tropically forced wave itself is sensitive to the existence of the upper level westerlies. For this forced stationary wave to propagate out of the tropics, westerlies are required, at least in a linear picture. If these westerlies are too weak, Rossby waves will have difficulty emerging from the tropics. As the westerlies increase, the tropically forced wave will emerge more easily, providing greater acceleration near the equator, which is required to get the ball rolling. The quantitative importance of this feedback is unclear. It does not appear to be required, for one can simply omit the zonally asymmetric heating and accelerate the upper troposphere with a prescribed zonally symmetric force. Varying the strength of this force, one still finds a similar bifurcation [17]. The feedbacks related to eddies generated in midlatitudes and in the tropics are illustrated schematically in Figure 12.

Secondly, the Hadley cell itself can feed back positively on the upper level equatorial westerlies. Given a torque at the equator, what provides the compensating deceleration in a steady state? Presumably the transport of momentum from the lower to the upper troposphere by the Hadley cell is the dominant factor:

$$
w \frac{\partial \bar{u}}{\partial z}(\theta=0) \approx \text { torque }
$$

However, as the westerlies increase, one expects the Hadley cell to decrease in strength. Upper level equatorial westerlies imply a warmer troposphere by thermal wind balance (taking into account the change in sign of $f$ at the equator). The adiabatic cooling at the equator must be reduced to be consistent with these warmer temperatures. (This is not the whole story, as one also expects the Hadley cell to shrink, since angular momentum conservation, starting with non-zero wind at the equator, will generate stronger temperature gradients). Again, it is not clear if this mechanism plays an important role in the kind of calculation pictured above. But in isolation this mechanism is capable of creating a bifurcation in axisymmetric models in which there are no large scale eddy stresses, as one varies the strength of a zonally symmetric equatorial torque [19].

In a moist atmosphere, one might expect superrotation to be harder to achieve if only because the Hadley cell is stronger, so one might imagine that the vertical advection opposing the westerly torques would be stronger. But this is not self-evident. The problem is that there really is no "large scale" upward motion in a moist Hadley cell. All of the upward motion is presumed to occur in small-scale convective towers. Between these towers, disregarding vertical motion associated with "synoptic" eddies of various kinds, we expect subsidence driven by radiative cooling. The implication is that there is no strong reason to believe that $w \partial \bar{u} / \partial z$, where $w$ is an average over some scale (say a few hundred kilometers), is a good model of momentum transport. The net effect of the convective motions and the environmental subsidence could be a flux that is smaller than the "large-scale" advective value; note that the environment alone transports momentum in the opposite sense. It is not clear that moist models should be less likely to superrotate than dry models. 

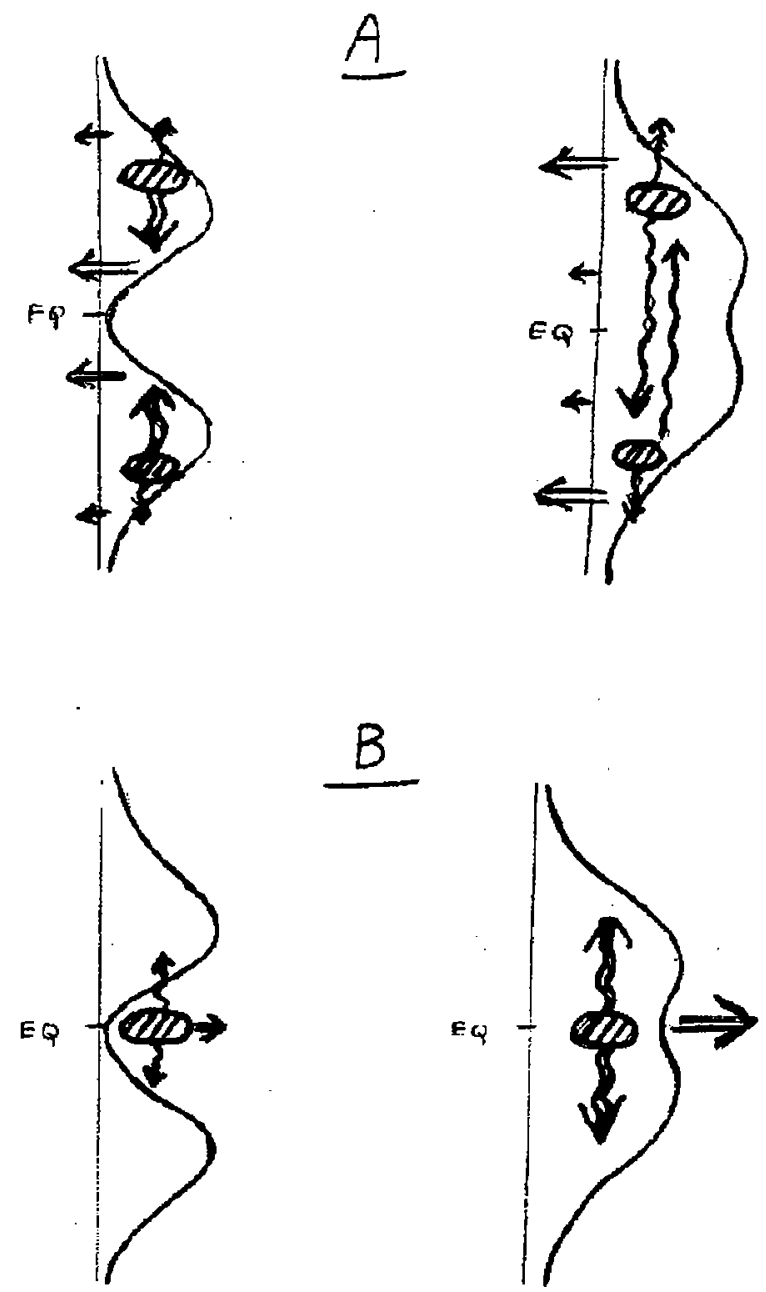

Figure 12: Schematic of the two eddy feedbacks 
In any case, there is as yet no evidence that comprehensive climate models undergo an abrupt bifurcation of this sort. Why this is so is unclear. It does seem that two-layer models are more susceptible to this bifurcation, so it may simply be a matter of vertical resolution, but there are no compelling arguments for why vertical resolution should matter that much. We often think in terms of two-level or two-layer models to help us get our minds around the complexity of the general circulation, hoping that we have not thrown out the baby with the bath water. The critical shear for baroclinic instability, which typically decreases to zero as the vertical resolution increases, is but one example of how one can be misled by thinking in terms of too idealized a model; the superrotation bifurcation may provide another example.

\section{Turbulent Diffusion in Midlatitudes}

Turning away from the tropics, we can no longer avoid the basic question of how the lower tropospheric eddy heat transport through midlatitudes is controlled. It is this heat transport that in turn controls the equator-to-pole temperature gradient on the Earth. As we have seen, given this heat transport we can also compute the equatorward mass flux in the "interrupted" surface layers, or the returning mass flux aloft (to within corrections due to the Ekman drift associated with the surface drag generated by eddy momentum fluxes, and assuming that we are given the tropospheric static stability). Equivalently, the heat flux is also the form drag that transfers angular momentum from the upper to the lower troposphere, angular momentum that is constantly replenished by transport from lower latitudes.

One can make some progress by thinking of these fluxes as diffusive

$$
\overline{v^{\prime} T^{\prime}} \sim-\mathcal{D} \frac{\partial T}{\partial y} .
$$

At the simplest level, this is just dividing the flux by the gradient, which leaves the convenient units of $(\text { length })^{2}($ time $)=($ velocity $)($ length $)$. The value of diffusivity one finds in midlatitudes is $1-2 \times 10^{6} \mathrm{~m}^{2} / \mathrm{s}$. The temperature field is not strongly distorted by the eddies in the lower troposphere, being rather strongly forced, so it seems plausible to think of the eddies as riding on a well-defined environmental gradient (as contrasted with the situation in the upper troposphere, where the eddies are more efficient at distorting and mixing the potential vorticity and where, therefore, one is not inclined to divide the flux by the gradient.) Perhaps more importantly, one can try to argue that finite amplitude baroclinic eddy production is fundamentally local and therefore effectively diffusive.

Think of a standard Benard convection problem: fluid confined between horizontal plates with fixed temperatures, and of horizontal extent much larger than the vertical extent. Diffusive theories cannot have any great value in this context because the scale of eddies transporting heat is determined by the vertical size of the domain, so there is no scale separation between eddies and mean flow. A theory for heat transport in such a geometry must be fundamentally global.

But now consider an alternative geometry, pictured in Figure 13, in which the aspect ratio of the the apparatus has been altered so that the horizontal scale is much smaller than the vertical scale. One might now expect that, in a turbulent flow, in which eddy 
statistics in different directions tend to be isotropized by nonlinear advection, that the small horizontal scale would set the dominant eddy scale. The result would be a separation of scales between this mixing length and the larger vertical scale imposed by the geometry. The heat flux through this kind of system would be diffusive in character. In particular, the resulting temperature profile, rather than being more or less homogenized in the interior might be more or less linear, as in the simplest solutions of the diffusion equation. The essential distinction is that, in this second case, the scale of the eddies is not determined by the scale of the mean inhomogeneity in the direction of the transport, as in most familiar turbulent flows, but rather by mean flow inhomogeneity in a direction perpendicular to the transport.

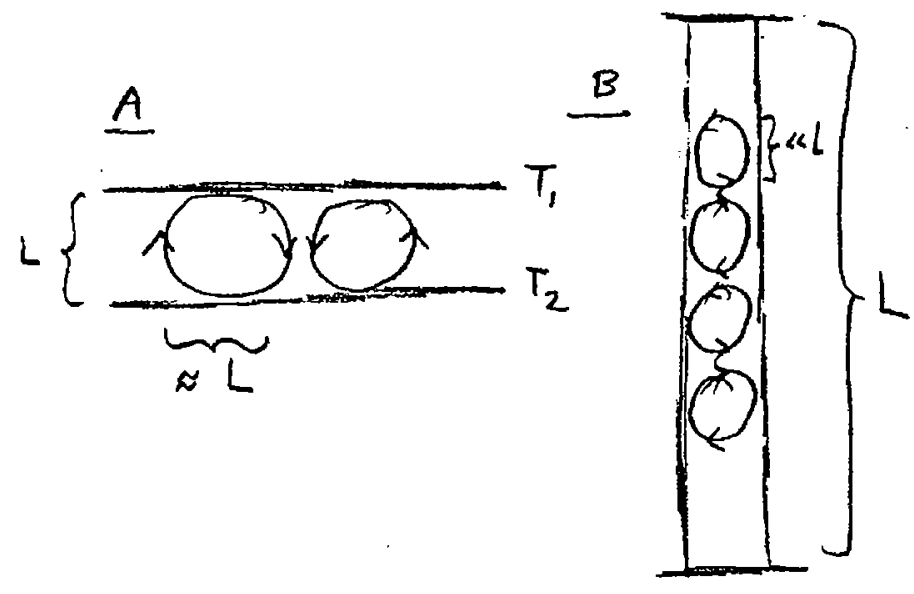

Figure 13: How turbulent convection in a domain with height/width $\ll 1$ is different from convection in a domain with height/width $\gg 1$; the latter is more "diffusive".

Why is baroclinic eddy production "diffusive" in this sense? In linear barotropic shear flows, the zonal scale of the instability is simply determined by the meridional scale of the mean shear variations. At finite amplitude, if sufficiently nonlinear, we expect the mixing length across the shear will also be of this scale. So no scale separation is possible here. In linear baroclinic problems, however, it is the vertical scale of the mean shears, multiplied by the Prandtl ratio $N / f$, that sets the zonal scale of the eddies. We speak of the zonal scale being the "radius of deformation" $N H / f$ where we can typically think of $H$ as proportional to the depth of the troposphere. Once again, we can expect nonlinear isotropization in the horizontal to turn this scale into a meridional mixing length. If $N H / f$ is small compared to the scale of horizontal inhomogeneity, then we do have the potential for scale separation. Even if the separation is not pronounced, we can hope, as in WKB theory, that the local theory is qualitatively useful even when the ratio of intrinsic to environmental scales is order unity.

To make this claim more precise, one can define a (numerical) apparatus to measure the diffusivity of turbulent baroclinic eddies as a function of the environmental gradients, just as one sets up a laboratory apparatus to measure the molecular diffusivity of a gas or the 
resistivity of a metal. Working in QG theory, the procedure is to specify the environmental potential vorticity gradients at each height, and then assume that the departures from these environmental values are doubly periodic in the horizontal:

$$
\frac{\partial q}{\partial t}=-J(\psi, q)-U \frac{\partial q}{\partial x}-\frac{\partial \psi}{\partial x} \frac{\partial Q}{\partial y}-D(q)
$$

Here $q$ and $\psi$ are doubly periodic, $U$ and $\partial Q / \partial y$ are the horizontally uniform environment, and $D$ is the dissipation. This equation for the eddies is horizontally homogeneous (but anisotropic). If the environment is unstable, eddies will grow and generate homogeneous geostrophic turbulence that will produce a downgradient flux of potential vorticity in the interior and a downgradient flux of heat along the lower surface. If one tried to set up such a problem for standard Benard convection, the model would "short circuit"; by maintaining the environment (analogous to maintaining a potential drop across a piece of metal) one is providing an infinite supply of energy. Only if a mixing length does develop will a meaningful answer emerge from such a geometry (just as the mean free path of electrons must be smaller than the apparatus if we are to measure the resistance of the metal rather than create an accelerator!) When we perform such a calculation, we find that baroclinic eddies do generate a mixing length that is independent of horizontal mean flow inhomogeneities.

Given the diffusivity determined by such a "theory", which will be dependent on the environmental shears and the form of the dissipation, we can then use this diffusivity in theories for the large-scale flow. Figure 14 provides some evidence that this kind of local diffusive theory actually works in some idealized cases (see [13]). A two-layer model, in which the interface slope is relaxed to some zonally symmetric radiative equilibrium, has been integrated to statistically steady state, for different values of the width of the unstable region. The upper level potential vorticity fluxes are shown as a function of latitude. Also shown are the fluxes obtained by using a diffusive flux closure obtained from a horizontally homogeneous diffusivity-measuring apparatus. (In this model, jet formation is not very strong, due in part to strong surface friction, so momentum fluxes are weak and potential vorticity fluxes in the upper layer are dominated by thickness fluxes.) Even when the baroclinic zone is only a few radii of deformation wide, the "theory" looks useful. But even if only qualitative, it provides a heuristic way of thinking of the problem as divided into two parts: a local theory for diffusivities; and the global response to the resulting eddy fluxes.

Let's focus on possible theories for the diffusivity. We need a velocity scale $V$ and a length scale $L$. A good starting point [20] is that $L \sim N H / f$ and $V \sim U \sim H \partial u / \partial z$, so that

$$
\mathcal{D} \sim \frac{N H^{2}}{f} \frac{\partial u}{\partial z}
$$

How can one justify the assumption that $V \sim U$, that the eddy kinetic energy is comparable to the zonal mean kinetic energy?

Baroclinic instability extracts available potential energy (APE) from the mean state. It is plausible that an eddy of scale $L$ can only extract the APE contained with a domain of scale $L$. In QG theory, defining the buoyancy $b=g \Theta / \Theta_{0}$ we have for this energy

$$
V^{2} \sim A P E \approx \frac{(\Delta b)^{2}}{N^{2}} \approx\left(\frac{L}{N} \frac{\partial b}{\partial y}\right)^{2} \approx\left(\frac{f L}{N} \frac{\partial u}{\partial z}\right)^{2}
$$




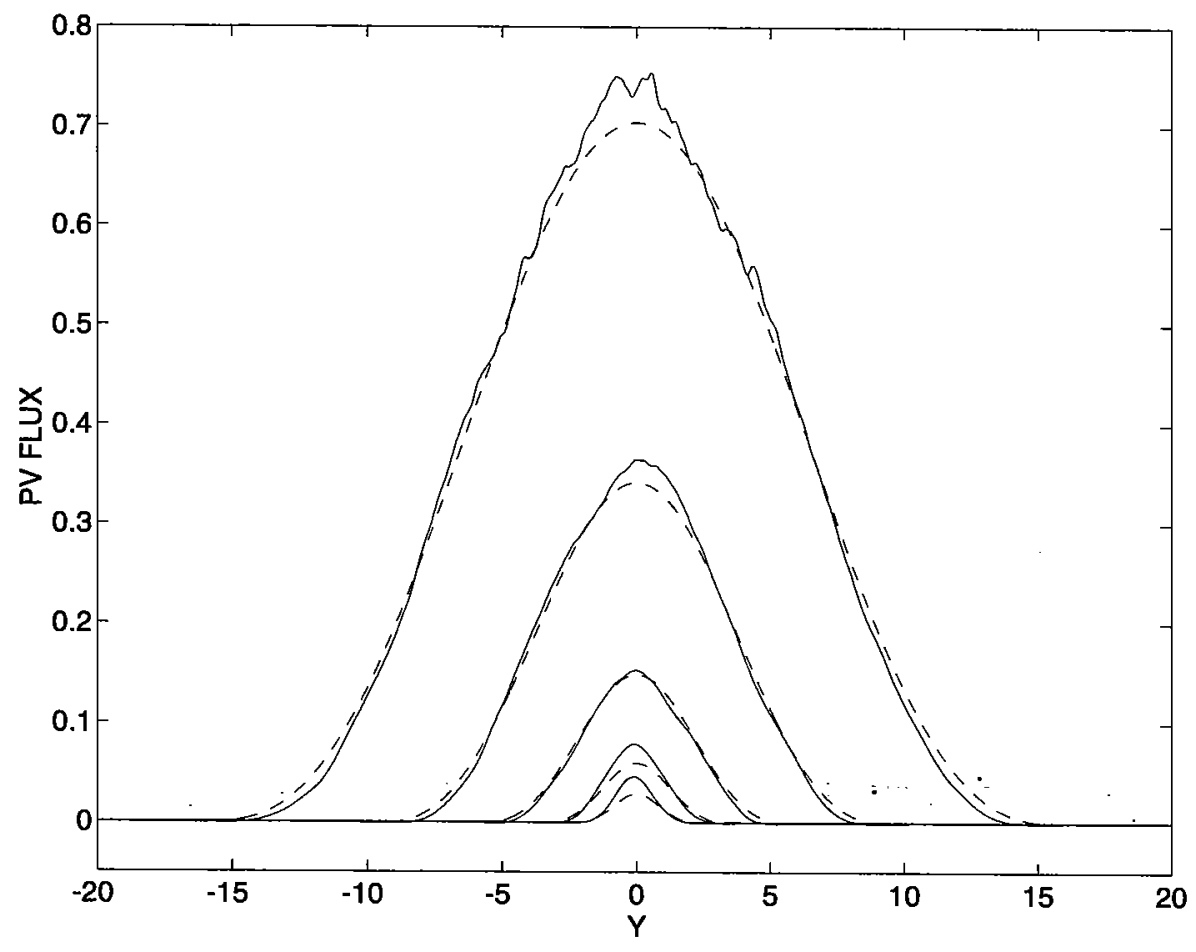

Figure 14: Solid: potential vorticity fluxes in the upper layer of a two-layer model. Dashed: diffusive closure theory with diffusivity determined numerically by integrating homogeneous model, from [13] 
If $L=\lambda \equiv N H / f$, this yields $V \sim U$ as desired. More generally,

$$
V \sim \frac{L}{\lambda} U
$$

(For an alternative derivation, see [5].) Whatever $L$, this expression for $V$ leads to the time scale

$$
T \sim \frac{L}{V} \sim \frac{\lambda}{U} \sim \frac{N}{f \partial U / \partial z} \sim \frac{R i^{1 / 2}}{f}
$$

which happens also to be the e-folding time for baroclinic instability in the Eady and Charney models. Note that $T$ is independent of $L$. By these arguments, the diffusivity is

$$
\mathcal{D} \sim \frac{L^{2}}{\lambda} U
$$

If $L \sim \lambda$, then the diffusivity is proportional to the horizontal temperature gradient, so the heat flux is proportional to the square of the temperature gradient. In this case the heat flux is also directly proportional to $N$, which is counterintuitive.

A familiar and fundamental property of two-dimensional flows is that energy cascades to larger horizontal scales, rather than smaller scales, as in 3D turbulence. In QG flows, the generic behavior is that the flow evolves to both larger vertical and horizontal scales, but that once the vertical scale has been maximized by the flow becoming more and more barotropic, the cascade to larger horizontal scales continues. If there is room and if nothing stops the cascade, then we expect $L>\lambda$.

There are a number of mechanisms that can stop the inverse energy cascade. One is surface friction. Suppose that surface friction results in a spin-down time of $\kappa^{-1}$. One might suspect that the time scale of the eddies would increase as $L$ increased, until this time scale reached $\kappa^{-1}$, at which point the cascade would stop. But this does not occur; the time scale is independent of $L$ ! This is a consequence of an important feedback: as the eddy length scale increases, the mixing length, the APE extracted by a typical eddy, and the eddy velocity scale all increase. So it does not appear that friction can stop this cascade. However, a better model of frictional damping in the atmosphere follows from assuming that the surface stress is quadratic in the geostrophic velocity, $\propto C_{D} V^{2}$, where $C_{D}$ is a non-dimensional drag coefficient. The value of $C_{D}$ depends on the roughness of the surface; typical values range from $10^{-3}$ over the ocean to $10^{-2}$ over very rough land surfaces. Since this stress must be distributed over the vertical depth $H$ to obtain an acceleration, the effective frictional time scale is

$$
\kappa^{-1} \sim \frac{H}{C_{D} V}
$$

Setting $\kappa T \sim 1$ yields the very simple result $L \sim H / C_{D}$ ! This scale is larger than the radius of deformation as long as $C_{D}$ is smaller than $f / N$. The resulting diffusivity would still be proportional to the temperature gradient, but independent of the static stability and proportional to $C_{D}^{-2}$

An alternative to stopping the inverse cascade with friction is to stop it, or slow it down, with the $\beta$-effect [16]. Longer Rossby waves have higher frequencies, so as the inverse cascade proceeds one has the potential to excite faster and faster waves. Once these frequencies become comparable to the inverse of the eddy time scale, one expects the nonlinear cascade 
to slow down and be replaced by more selective anisotropic interactions. Equating the Rossby wave frequency $\beta L$ with $T^{-1}$ results in $L \sim 1 /(\beta T)$ and a diffusivity

$$
\mathcal{D} \sim \beta^{-2} T^{-3} \text {. }
$$

This diffusivity has a very strong cubic dependence on horizontal temperature gradient, resulting in a heat flux proportional to the fourth power (!) of the gradient. Experiments with horizontally homogeneous two-layer models on a $\beta$-plane, with linear friction, show a dependence of the flux on the temperature gradient which, if anything, is even stronger than this [5]. The expression for $L$ can be rewritten as

$$
\frac{L}{\lambda} L \sim \frac{U}{\beta \lambda^{2}} \sim \frac{a}{H} I_{\Theta}
$$

where $I_{\Theta}$ is the isentropic slope and we have evaluated $f$ and $\beta$ in midlatitudes. If the isentropic slope is such that it takes one from the top to the bottom of the troposphere as one moves from the pole to the equator (as observed), then we evidently should not expect to cascade to larger scales than the radius of deformation before we are stopped by $\beta[18]$.

These arguments are too crude to determine whether quadratic friction or the $\beta$-effect are dominant in stopping the cascade in the atmosphere (both are probably important). If nothing stops the cascade before its generates mixing lengths comparable to the radius of the Earth, we can set $L \sim a$, which yields the diffusivity proposed in [3].

\section{The Extratropical Static Stability}

In addition to the amplitude of the eddy fluxes, we also need to understand the vertical structure of the potential vorticity fluxes in order to discuss the maintenance of the extratropical static stability. How high does the eddy potential vorticity mixing extend? Much of this mixing admittedly occurs near the tropopause, partly because the eddies have been relatively successful at homogenizing the mid-troposphere. (These waves have steering levels in the mid-troposphere, so this is where the largest meridional particle displacements are to be expected). But what determines the height of the tropopause?

To understand what some of the issues are, consider a Boussinesq atmosphere with no upper boundary and with uniform $N(z)$ and uniform vertical shear $\bar{u}=\Lambda z$ on a $\beta$-plane. The potential vorticity gradient in the interior is simply $\beta$, with the surface temperature gradient creating baroclinic instability. What is the horizontal scale of the most unstable wave in this system? One is tempting to say $N h / f$, but what is the appropriate vertical scale $h$ ? The answer is

$$
h \approx \frac{f^{2} \Lambda}{\beta N^{2}} ; L \approx \frac{f \Lambda}{\beta N}
$$

One can obtain this answer in several ways: by examining the linear system and nondimensionalizing (there is only one way of doing this); or by using the counter-propagating Rossby wave picture of baroclinic instability (which we haven't discussed here, see [9]) and finding that height $h$ at which a Rossby wave with scale $N h / f$ would propagate westward with the right phase speed so as to match the eastward propagation of the "Eady edge wave" with respect to the flow at the ground. 
Notice that the vertical penetration of the wave is proportional to the vertical shear, or, more precisely, to the isentropic slope: $h \approx f I_{\Theta} / \beta \approx I_{\Theta} a$. The eddies adjust themselves so that the fractional variations in buoyancy, or potential temperature, in the vertical over the scale of the wave itself are comparable to the variations in the horizontal over the scale of the planet!

There is an analogous barotropic problem, the eastward point jet on a $\beta$-plane. Here the flow is simply $\bar{u}=\Lambda|y|$. The most unstable wave in this system has the scale $\Lambda / \beta$ in $x$ and extends roughly this same distance in $y$. The baroclinic result is identical except that it is scaled by the Prandtl ratio $f / N$ as appropriate.

What is the relevance of this argument for statistically steady states, as opposed to linear theory? Consider the barotropic jet problem first. The eddies will try to stabilize the flow, but if we use the Rayleigh-Kuo necessary condition for instability as a guide, to do this they need to remove the destabilizing curvature at $y=0$. What is the minimum distance out to which vorticity must be homogenized so at to insure that the vorticity gradient is no longer negative? If we homogenize the vorticity up to distance $\Lambda / \beta$ and assume continuity of $\bar{u}$ we can remove the negative vorticity gradient, but this requires the mean flow to be accelerated everywhere, which does not conserve momentum. To conserve momentum, we have to mix somewhat farther, but, in any case, this distance scales like $\Lambda / \beta$. We can refer to this as "barotropic adjustment", and can picture the eddies trying to achieve this state and coming to some accommodation with the forcing of the mean flow in which these same scales remain relevant. The same picture can be drawn for the baroclinic problem by simply changing $y$ to $z$, even though the nonlinear dynamics is profoundly different, (and we need only that part of the domain with $z>0$ ) resulting in a construction that can be thought of as the continuous version of "baroclinic adjustment" ([10]), in which the eddies try to destroy the surface temperature gradient and the potential vorticity gradients up to the height $h$. (They do not succeed entirely, especially near the surface where the forcing is too strong, but also at upper levels if a jet is present which will encourage mixing on its flanks and the formation of a mixing barrier at the jet core, as in Figure $\dot{8}$.)

The scaling in (54) assumes a Boussinesq atmosphere. Once this scale becomes comparable to the scale height $H$, dimensional arguments alone are insufficient to determine how these scales depend on $h / H$. The baroclinic adjustment construction can be generalized to the non-Boussinesq case; the depth of the eddy fluxes increases logarithmically with increasing horizontal temperature gradient, rather than linearly as above. Whether or not this prediction is reliable is unclear.

Given a theory for the vertical extent of the baroclinic eddy fluxes, can we complete our picture by providing a theory for the tropospheric static stability?

We have to return at this point to thinking about radiative forcing, which after all is ultimately driving everything. Think of the atmospheric absorber distribution as fixed for simplicity. Pure radiative equilibrium produces a very unstable vertical temperature profile. One simple way of fixing this is with a convective adjustment. Whenever the tropospheric lapse rate exceeds the dry adiabatic value, adjust it back to this value while conserving energy. This requires a vertical energy flux. The result of this construction is a "troposphere" extending from the ground up to some height $H_{T}$, in which the lapse rate is the dry adiabatic value, bounded above by a "stratosphere" in radiative equilibrium. Now suppose one has a theory for how the circulation maintains a lapse rate that is more 
stable than this dry adiabatic value. One can use this value in one's adjustment algorithm and generate a model in which the troposphere is forced to possess this prescribed static stability. As shown in Figure 15, this will result in a deeper troposphere. In general, one can think of $H_{T}$ as a function of $N$. We can refer to this as the "radiative constraint" between $H_{T}$ and $N$.

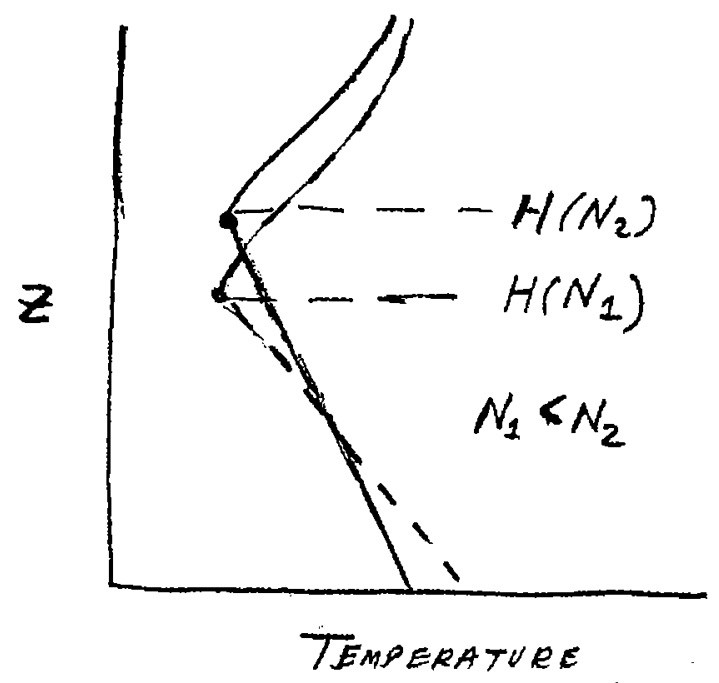

Figure 15: Schematic of temperature profiles obtained from a convective-adjustment algorithm, for different values of the prescribed tropospheric static stability

We need another relation, a dynamical constraint, between these two parameters in order to determine their values. Usually this kind of convective adjustment model is applied to the global mean temperature profile, but we can usefully apply it to the tropics and extratropics separately; indeed we must, since the kinds of eddies transporting heat vertically are so different in these two regions. (Technically, we now need to include the transport of heat from the tropics to midlatitudes in our computations, in both atmosphere and ocean; formally, we can just bundle these fluxes together with the radiative forcing, but this does not change the underlying picture.)

In the tropics, moist convection is dominant in the vertical energy fluxes. We can assume that moist convection more or less forces the moist static energy near the tropopause to be close to that near the surface in the most strongly convecting regions, or, averaged over the troposphere,

$$
N^{2} \approx \frac{L q(0)}{c_{p} T(0)} \frac{g}{H_{T}}
$$

which serves as our dynamical constraint. We can think of the mixing ratio for water vapor near the surface, $q(0)$, as given, or, better, we can imagine computing it from the surface temperature predicted by our radiative-convective model, assuming saturation close to the ground. Figure 16 illustrates how the radiative and dynamical constraints would then combine to determine $N$ and $H_{T}$. 


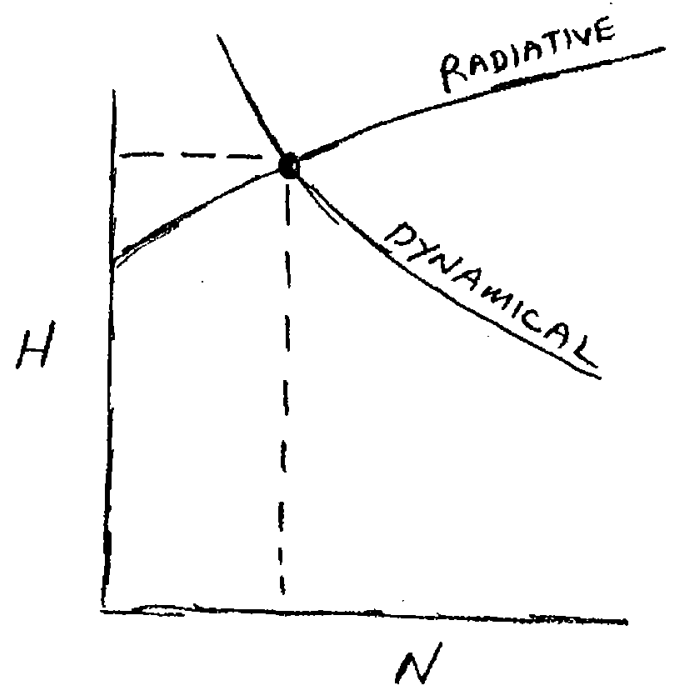

Figure 16: The radiative and dynamical constraints combine to determine the tropospheric static stability and the height of the tropopause.

In the extratropics, we need an analogous theory for the depth of penetration of the dynamical heating as a function of $N$. A theory of the type outlined above, leading to the non-Boussinesq generalization to (54) is a candidate for the appropriate constraint. It once again predicts that the tropopause height increases with decreasing static stability, so the resulting picture is similar to that above.

To summarize, in midlatitudes the low level eddy heat flux is more or less diffusive, with a diffusivity that depends on horizontal and vertical potential temperature gradients and on the strength of surface drag. Horizontal temperature gradients are determined by the balance between these diffusive fluxes and the radiative driving, as in the simplest diffusive energy balance models. Given this horizontal gradient, the tropopause height and the tropospheric static stability adjust to simultaneously satisfy the radiative and dynamical constraints, with both feeding back on the diffusivity to obtain a self-consistent picture. The missing ingredient that we have not discussed is the effect of moist convection in the warm sectors of cyclones on both the static stability of the troposphere and on eddy diffusivities.

In the tropics, vertical structure can be determined by a radiative-convective model in which the adjustment is made to the moist adiabat, using moisture levels from the region with the largest moist static energy, or moist entropy, at low levels. A circulation is generated so as to maintain small temperature gradients in the free troposphere, the width of this tropical circulation being determined by the region within which, in its absence, the upper levels winds would be too large to be physical realizable. This circulation forces the entire tropics to lie close to this moist adiabat and to possess the same tropopause height, resulting in a discontinuity in the tropopause at the subtropical jet. The inconsistency between this moist adiabat and surface temperatures is taken up at the trade wind inversion. 


\section{References}

[1] Andrews, D. G., and M. E. McIntyre, 1976: Planetary waves in horizontal and vertical shear. The Generalized Eliassen-Palm relation and the mean zonal acceleration. $J$. Atmos. Sci., 33, 2031-2048.

[2] Edmon, H. J., B. J. Hoskins, and M. E. McIntyre, 1980: Eliassen-Palm cross sections for the troposphere. J. Atmos. Sci., 37, 2600-2616. Erratum: 38, 1115.

[3] Green, J. S. A., 1970: Transfer properties of the large-scale eddies and the general circulation of the atmosphere. Quart. J. Roy. Meteor. Soc., 96, 157-185.

[4] Held, I. M. and A. Y. Hou, 1980: Nonlinear axisymmetric circulations in a nearly inviscid atmosphere. J. Atmos. Sci., 37, 515-533.

[5] Held, I. M., and V. D. Larichev, 1996: A scaling theory for horizontally homogeneous, baroclinically unstable flow on a $\beta$-plane. J. Atmos. Sci., 53, 946-952.

[6] Held, I. M., and T. Schneider, 1999: The surface branch of the zonally averaged mass transport circulation in the troposphere. J. Atmos. Sci., 56, 1688-1697.

[7] Held, I. M., and M. J. Suarez, 1994: A proposal for the intercomparison of the dynamical cores of atmospheric general circulation models. Bull. Am. Meteor. Soc., $\mathbf{7 5}$, 1825-1830.

[8] Hoskins, B. J., H. H. Hsu, I. N. James, M. Masutani, P. D. Sardeshmukh, and G. H. White, 1989: Diagnostics of the Global Atmospheric Circulation based on ECMWF Analyses 1970-1980. WCRP-27, WMO/TD No. 326, 217 pp.

[9] Hoskins, B. J., M. E. McIntyre, A. W. Robertson, 1985: On the use and significance of isentropic potential vorticity maps. Quart. J. Roy. Meteor. Soc., 111, 877-946.

[10] Lindzen, R. S., and B. Farrell, 1980: The role of polar regions in global climate; and a new parameterization of global heat transport. Mon. Weather. Rev., 108, 2064-2079

[11] Palmen, E., and C. W. Newton, 1969: Atmospheric Circulation Systems, Academic Press, New York, 603pp.

[12] Panetta, R. L., 1983: Zonal jets in wide baroclinically unstable regions: persistence and scale selection. J. Atmos. Sci. 40, 2073-2106.

[13] Pavan, V., and I. M. Held, 1996: The diffusive approximation for eddy fluxes in baroclinically unstable jets. J. Atmos. Sci., 53, 1262-1272.

[14] Peixoto, J. P. and A. H. Oort, 1992: Physics of Climate. American Institute of Physics, New York, pp. 520.

[15] Randel, W. J., and I. M. Held, 1991: Phase speed spectra of transient eddy fluxes and critical layer absorption. J. Atmos. Sci., 48, 688-697.

[16] Rhines, P. B., 1975: Waves and turbulence on a $\beta$-plane. J. Fluid Mech., 69, 417-443. 
[17] Saravanan, R., 1993: Equatorial superrotation and maintenance of the general circulation in two-level models, J. Atmos. Sci. 50, 1211-1227.

[18] Schneider, T. , personal communication, 1998.

[19] Shell, K., this volume

[20] Stone, P. H., 1972: A simplified radiative-dynamical model of the static stability of rotating atmospheres. J. Atmos. Sci., 29, 405-418.

[21] Suarez, M. J. , and D. G. Duffy, 1992: Terrestrial superrotation: a bifurcation of the general circulation. J. Atmos. Sci. 49, 1541-1554.

[22] Williams, G. P., and J. L. Holloway, Jr., 1982: The range and unity of planetary circulations. Nature, 297, 295-299. 


\title{
Monsoons in a Moist Axially Symmetric Model of the Atmosphere
}

\author{
Giulio Boccaletti
}

\section{Introduction}

Large scale meridional overturning circulations such as the Asian monsoon and the Hadley Cell are a dominant feature of the dynamics of the tropical atmosphere.

Our theoretical understanding of these circulations is largely based on idealized axisymmetric models, which describe them as steady-state and quasi-inviscid. In such theoretical models it can be shown [1] that meridional flow can only occur if it conserves angular momentum, entailing a condition on the thermal forcing of the atmosphere.

In the case of the Asian monsoon, Xie and Saiki [2] have recently argued that this constraint can be only met once moisture has penetrated over the Indian sub-continent, and that this is the result of a zonally asymmetric instability that draws water over land from the Indian Ocean, marking the onset of the monsoon.

Their argument entails that differences in vertical thermal structure between a moist convecting atmosphere and a dry one are critical for the existence of a monsoon. Furthermore, an axisymmetric model should be unable to produce a monsoon, as zonally asymmetric disturbances are absent.

To verify whether this is indeed the case, we construct an axisymmetric model of the atmosphere with interactive moist convection. The objective is to achieve a reasonable representation of the effects of moist convection on the large scale dynamics of the tropical atmosphere, therefore improving previous idealized models, in which typically a vertical thermal structure is externally imposed $[3,1]$.

Section (2) is devoted to the formulation of the model. Section (3) will analyze the results of simulations of monsoonal flow and section (4) will draw the conclusions. 


\section{The model}

\subsection{The averaged equations}

Consider the following axially symmetric equations for a hydrostatic atmosphere:

$$
\begin{aligned}
\frac{\partial u}{\partial t}+\frac{v}{a^{2} \cos (\theta)} \frac{\partial M}{\partial \theta} & =-\epsilon u \\
\frac{\partial v}{\partial t}+f u+\frac{u^{2}}{a} \tan (\theta) & =-\frac{1}{a} \frac{\partial \Phi}{\partial \theta}-\epsilon v \\
\frac{\partial \Phi}{\partial \pi} & =-C_{p} \Theta \\
\frac{\partial \dot{\pi}}{\partial \pi}+\frac{1}{a \cos (\theta)} \frac{\partial v \cos (\theta)}{\partial \theta} & =0 \\
\frac{\partial \Theta}{\partial t}+\frac{v}{a} \frac{\partial \Theta}{\partial \theta}+\dot{\pi} \frac{\partial \Theta}{\partial \pi} & =Q
\end{aligned}
$$

where $\pi=\left(\frac{p}{p_{0}}\right)^{R / C_{p}}$ is the Exner pressure, $\Theta$ is the potential temperature, $\Phi$ is the geopotential, $M=\Omega a^{2} \cos (\theta)^{2}+u a \cos (\theta)$ is the angular momentum density, $(u, v, \dot{\pi})$ is the $3 \mathrm{D}$ velocity vector, $f=2 \Omega \sin (\theta)$ is the Coriolis parameter, $\theta$ is the latitude, $\epsilon$ is a Rayleigh friction, and $Q$ is the diabatic heating. All variables are intended as large scale variables, resulting from an appropriate average which requires parameterization of organized convection.

Vertical advection of momentum and horizontal advection of meridional momentum have been ignored, based on the sole assumption that they are small for the flows that we are concerned with and therefore only quantitatively important for the problem.

To first approximation a monsoon such as the Asian monsoon is a meridional overturning circulation, with an upper branch uniformly flowing through most of the troposphere, the return flow being confined to a relatively thin surface mixed layer. We can therefore divide our model troposphere in two layers: one layer of thickness $\pi_{M L}-\pi_{t o p}$, representing the free atmosphere, overlaying a layer of thickness $\pi_{0}-\pi_{M L}$ representing the mixed layer. $\pi_{0}$, $\pi_{M L}, \pi_{t o p}$ are the specified pressure heights of the surface, the interface between the mixed layer and the free atmosphere, and the top of the troposphere respectively.

Integrating equation (3) vertically we obtain for the two layers:

$$
\left\{\begin{array}{c}
\Phi_{M L}-\Phi_{t o p}=-C_{p} \int_{t o p}^{M L} \Theta d \pi \\
\Phi_{0}-\Phi_{M L}=-C_{p} \int_{M L}^{0} \Theta d \pi
\end{array}\right.
$$

so that the geopotential thickness is the vertically integrated temperature profile (for convenience we set $\Phi_{0}=0$ ).

If we impose

$$
\pi_{M L}-\pi_{t o p} \gg \pi_{0}-\pi_{M L}
$$

it is reasonable to assume that

$$
\Phi\left(\pi_{\text {top }}\right) \gg \Phi\left(\pi_{M L}\right)
$$


We will also assume that

$$
\frac{\partial \Phi\left(\pi_{t o p}\right)}{\partial \theta} \gg \frac{\partial \Phi\left(\pi_{M L}\right)}{\partial \theta}
$$

which for an atmosphere close to geostrophy is equivalent to

$$
u\left(\pi_{t o p}\right) \gg u\left(\pi_{M L}\right) .
$$

For zonal wind at the top of the troposphere the pressure force in equation (2) is then simply

$$
\frac{1}{a} \frac{\partial \Phi_{t o p}}{\partial \theta}=\frac{g}{a} \frac{\partial h}{\partial \theta}
$$

where

$$
h=\frac{C_{p}}{g} \int_{\pi_{t o p}}^{\pi_{M L}} \Theta d \pi
$$

In each layer the meridional velocity $v$ is assumed constant, so that equations (1), (2) with the pressure force given by equation (11) are momentum equations for the meridional flow in the free atmosphere and zonal flow at the top of the troposphere. The system of equations must be closed with a theory for $h$.

\subsection{The thermodynamic equation and the convective closure}

Vertically integrating equation (5) in the free atmosphere gives:

$$
\frac{\partial h}{\partial t}+\frac{v}{a} \frac{\partial h}{\partial \theta}+\frac{C_{p}}{g} \int \dot{\pi} \frac{\partial \Theta}{\partial \pi} d \pi=\frac{C_{p}}{g} \int Q d \pi
$$

The third term on the l.h.s. of equation (13) can be integrated by parts to obtain

$$
\int \dot{\pi} \frac{\partial \Theta}{\partial \pi} d \pi=\int \frac{\partial \dot{\pi} \Theta}{\partial \pi} d \pi-\int \frac{\partial \dot{\pi}}{\partial \pi} \Theta d \pi=\int \frac{\partial \dot{\pi} \Theta}{\partial \pi} d \pi+\frac{g}{C_{p}} \frac{h}{a \cos (\theta)} \frac{\partial v \cos (\theta)}{\partial \theta}
$$

where the last equality has been derived using the continuity equation (4) and the fact that we have assumed meridional wind independent of height.

Assuming $\dot{\pi}_{\text {top }}=0$ we obtain

$$
\int \frac{\partial \dot{\pi} \Theta}{\partial \pi} d \pi=\dot{\pi}_{M L} \Theta_{M L}
$$

where $\dot{\pi}_{M L}$ can be calculated from the continuity equation (4) to be

$$
\dot{\pi}_{M L}=-\int_{\pi_{\text {top }}}^{\pi_{M L}} \frac{1}{a \cos (\theta)} \frac{\partial v \cos (\theta)}{\partial \theta} d \pi=-\frac{1}{a \cos (\theta)} \frac{\partial v \cos (\theta)}{\partial \theta}\left(\pi_{M L}-\pi_{t o p}\right)
$$

Finally, substituting equations (14), (16) into (13) we get:

$$
\frac{\partial h}{\partial t}+\frac{v}{a} \frac{\partial h}{\partial \theta}+\left(h-\frac{C_{p}}{g} \Theta_{M L}\left(\pi_{M L}-\pi_{t o p}\right)\right) \frac{1}{a \cos (\theta)} \frac{\partial v \cos (\theta)}{\partial \theta}=\frac{C_{p}}{g} \int Q d \pi
$$

The term $\left(h-\frac{C_{p}}{g} \Theta_{M L}\left(\pi_{M L}-\pi_{t o p}\right)\right)$ is a measure of the dry static stability of the atmosphere, which varies as the integrated temperature changes: if the upper layer (i.e. the free 
atmosphere) is heated up then $h$ will increase and the amount of energy necessary to lift a unit thickness slab of atmosphere in a unit time will increase accordingly.

The diabatic forcing $Q$ represents the effect of moist convection, radiative cooling and unresolved turbulent fluxes on the temperature of the free atmosphere. In the absence of large scale circulation $(v=0)$ radiative cooling and convection would compete, driving the atmosphere towards a state of radiative-convective equilibrium.

We will assume that such a state is defined by a specific thermal structure so that the value of CAPE is fixed to zero:

$$
C A P E=\int_{p_{T O P}}^{p_{0}}\left(\alpha_{p}-\alpha_{e}\right) d p \simeq 0
$$

with $\alpha_{p}$ is the specific parcel volume and $\alpha_{e}$ is the specific volume of the environment, so that the atmosphere is neutrally buoyant ${ }^{1}$.

We will then require that the model satisfy equation (18) wherever convection, whether dry or moist, occurs. This means that the temperature profile in the free atmosphere will be that deduced by the pseudo-adiabatic lifting of a parcel of air from the mixed layer: in the absence of large scale circulation, the clear atmosphere relaxes to radiative equilibrium on a timescale of about 10 days; this profile of temperature is unconditionally unstable for normal surface temperatures so that convection develops spontaneously; parcels therefore rise following a dry adiabat up to the lifting condensation level (LCL) whence they follow a pseudoadiabat up to where their buoyancy meets that of radiative equilibrium. Radiative cooling always drives back the atmosphere to an unstable profile, so, as the convective process is much faster than radiative cooling, the temperature profile in a convecting region should be close to that traced by a particle lifted pseudo-adiabatically.

We now assume that in the presence of convection all vertical mass flux originates in the mixed layer: knowledge of the temperature and moisture in the mixed layer is then sufficient to determine the vertical temperature structure of a convecting region.

It follows that. we expect to substitute the r.h.s. of equation (17) with a relaxation towards the integrated temperature profile of a convecting atmosphere, calculated using the definition of equation (12). The problem has been reduced to determining the values of moisture and potential temperature in the mixed layer.

Axisymmetry requires that the meridional mass flow in the mixed layer be equal and opposite to that in the free atmosphere, so that at any latitude the meridional mass transport is zero. Therefore the velocity in the mixed layer is:

$$
v_{M L}=\frac{\Delta \pi_{\text {free }}}{\Delta \pi_{M L}} v
$$

A source of moisture is geographically specified by restoring (typically over the ocean) to saturation. The moisture budget is closed by including advection by the large scale flow as well as drying of the mixed layer by entrainment of dry air from above, so that the equation for moisture in the mixed layer is:

$$
\frac{\partial q}{\partial t}+\frac{1}{a \cos (\theta)} \frac{\partial q v_{M L} \cos (\theta)}{\partial \theta}=\left\{\begin{array}{cl}
0 & \text { if over land } \\
\frac{q_{s}-q}{\tau_{m}} & \text { if over ocean }
\end{array}\right.
$$

\footnotetext{
${ }^{1}$ There is some observational evidence that $C A P E \simeq 0$ might be the right assumption but the subject matter is controversial [4].
} 
where $q$ is the specific humidity of the mixed layer. The relaxation towards saturation $q_{s}$ has a timescale $\tau_{m}$ of typically 1-2 days. All condensation is assumed to occur above the mixed layer and notice that the balance doesn't explicitly contain precipitation. $q_{s}$ is a function of the temperature of the mixed layer alone, and therefore a function of the surface temperature, as the model is forced by a specified surface temperature, and we assume that the entire mixed layer has potential temperature equal to the imposed surface temperature.

Equation (20) is a crude approximation to the moisture budget. The model does not store soil moisture, so evaporation and precipitation over land are not part of the balance. This ensures that the ocean is the only moisture source for the model, a limitation that could be overcome in future work by allowing a more sophisticated model for the hydrology.

Convection will not always occur. Whenever the large scale circulation makes the free atmosphere more buoyant than convection would, convection must be inhibited. In this simplified model this occurs when $h$, the vertically integrated temperature of the free atmosphere, is greater than the vertically integrated temperature of a convecting atmosphere, with the same moist properties as the local mixed layer. In these non convecting regions the atmosphere must be relaxing towards radiative equilibrium with a timescale of about 10 days. This situation is equivalent to a region of trade inversion in the real atmosphere.

Given all the above, the following closure for moist convection is adopted:

$$
\frac{C_{p}}{g} \int \bar{Q} d \pi=\frac{H_{e}-h}{\tau_{h}(\theta)}
$$

with

$$
\left\{\begin{array}{lll}
H_{e}=\frac{C_{p}}{g} \int \Theta_{p}(\pi) d \pi, & \tau_{h} \simeq 6 \text { hours } \quad \forall h<\frac{C_{p}}{g} \int \Theta_{p}(\pi) d \pi \\
H_{e}=\frac{C_{p}}{g} \int \Theta_{r}(\pi) d \pi, & \tau_{h} \simeq 10 \text { days } \quad \forall h>\frac{C_{p}}{g} \int \Theta_{p}(\pi) d \pi
\end{array}\right.
$$

where $\Theta_{p}=\Theta_{p}(\pi)$ is the profile of potential temperature followed by a parcel rising pseudoadiabatically from the mixed layer, and $\Theta_{r}$ is a radiative equilibrium potential temperature profile for the free atmosphere which we assume constant at $\Theta_{r} \simeq 220^{\circ} \mathrm{K}$. Notice that the condition for convection amounts to a comparison of the dry static energy of the free atmosphere, as measured by the integrated temperature, with the moist static energy of the mixed layer. Therefore because moisture is not predicted in the free atmosphere the conditions more stringent than it should be.

No mention is explicitly given to large scale convergence or vertical velocity in the convective parameterization. Although precipitation is not thermodynamically coupled to the flow, potential for a feed-back between moist convergence and large scale circulation does exist, given the appropriate initial conditions, as will be seen in section (3.2) 
The final equations for the free atmosphere then take the form:

$$
\begin{aligned}
\frac{\partial u}{\partial t}+\frac{v}{a^{2} \cos (\theta)} \frac{\partial M}{\partial \theta} & =-\epsilon u \\
\frac{\partial v}{\partial t}+f u+\frac{u^{2}}{a} \tan (\theta) & =-\frac{g}{a} \frac{\partial h}{\partial \theta}-\epsilon v \\
\frac{\partial h}{\partial t}+\frac{v}{a} \frac{\partial h}{\partial \theta}+\left(h-\frac{C_{p}}{g} \Theta_{M L}\left(\pi_{M L}-\pi_{t o p}\right)\right) & \\
\cdot\left(\frac{1}{a \cos (\theta)} \frac{\partial v \cos (\theta)}{\partial \theta}\right) & =\frac{H_{e}-h}{\tau}
\end{aligned}
$$

$\tau$ is now a function of the convective state of the model, varying between a few hours and 10 days. $H_{e}$ is the integral of the restoring temperature profile, which is either the specified radiative equilibrium, or the convective value, dependent on the local values of temperature and moisture in the mixed layer.

Two different processes besides advection can change the integrated temperature in equation (25): one is the term on the r.h.s: representing convection and physical processes, and the other is the last term on the l.h.s. representing the stabilization/destabilization of a convergent/divergent large scale circulation. We will return to the relative magnitude and importance of these two processes in the last section.

\subsection{Steady state solutions in the limit of no viscosity}

In the limit of small viscosity, the solution to equations (23), (24), (25) becomes independent of the actual numerical value of $\epsilon$. A small but finite $\epsilon$ is nevertheless necessary for numerical stability, so a timescale of 400 days (much greater than any other timescale in the problem) is chosen for $\epsilon^{-1}$.

Notice that Hide's theorem as stated in [3] cannot be exactly applied in this case. Equation (23) in steady state flux form is

$$
\frac{\partial v M \cos (\theta)}{\partial \theta}=M \frac{\partial v \cos (\theta)}{\partial \theta}-\epsilon u a^{2} \cos (\theta)
$$

so that for a sufficiently small domain including a maximum of $\mathrm{M}$ the integral of the l.h.s. of equation (26) is zero. The integral of the r.h.s. is also zero if the local maximum of angular momentum density coincides with a divergent flow field (a mass source for the free atmosphere) so Hide's theorem does not hold and a maximum of $\mathrm{M}$ in the free atmosphere is possible. The problem arises from neglecting the vertical advection of momentum. However, a localized maximum of angular momentum density off the equator would be inertially unstable to small perturbations so that when integrating the time dependent equations such a solution would not occur. It has to be noted however that as formulated the model is not incompatible with equatorial superrotation.

Only steady state solutions of equations (23), (24), (25) will be considered here. As described in [1] two solutions exist for the zonal wind profile in the steady state inviscid limit: one in thermal wind equilibrium with the forcing and one angular momentum conserving. The former implies no meridional circulation while the latter implies a strong meridional 
circulation. Both are solutions of the approximate equation

$$
\frac{\partial M}{\partial \theta} v \sim 0
$$

Accordingly, the steady state form of equation (25) can be satisfied in one of two ways:

$$
\begin{gathered}
h(\theta)=H_{e}(\theta) \\
\text { ii) } \int\left[\left(\frac{C_{p}}{g} \Theta_{M L}\left(\pi_{M L}-\pi_{t o p}\right)\right) \cdot\left(\frac{1}{a \cos (\theta)} \frac{\partial v \cos (\theta)}{\partial \theta}\right)+\frac{H_{e}-h}{\tau}\right] d \theta=0
\end{gathered}
$$

equation (28) corresponding to $v=0$, and equation (29) corresponding to an integral balance which determines the extent and strength of the meridional circulation.

Whether the solution is of the first or second type depends on the strength of the forcing. Suppose a distribution of $H_{e}$ is imposed. The adjustment from rest will involve a progressive increase of $\mathrm{h}$ towards $H_{e}$. In doing so a pressure a pressure gradient will be established and a meridional circulation will start, which will then produce a zonal wind in geostrophic equilibrium with the pressure gradient. As $h$ increases the flow goes through successive quasi-equilibrium states of geostrophy. If $H_{e}$ does not involve too steep gradients, $h$ eventually reaches $H_{e}$ and the equilibrium solution is attained with no meridional flow. If on the other hand $H_{e}$ does involve steep gradients, the meridional flow involved in the adjustment can be strong enough that the equilibrium expressed by equation (29) is reached before (28) is achieved and a steady state meridional circulation results, which will be angular momentum conserving so that $\frac{\partial M}{\partial \theta}=0$.

A condition on $H_{e}$ can be calculated to discriminate between the two solutions. The condition, an expression for which can be found in [1], states that a meridional circulation will be a steady state solution if the imposed $H_{e}$ were to produce a geostrophycally adjusted zonal wind such that $\frac{\partial M}{\partial \theta} \geq 0$ in the northern hemisphere. This is equivalent to requiring that the flow be on the verge of inertial instability, although, as the adjustment sequence described above implies, inertial instability is actually never reached.

This description is inadequate for our model as $H_{e}$ cannot be imposed: it depends instead on the moisture distribution in the mixed layer which is predicted by the model. We therefore turn to numerical integration.

\section{The monsoon}

The model described by equations (23), (24), (25), (20) with condition (22) was discretized on a staggered grid with meridional resolution of 200 points, corresponding to slightly less than one degree per grid box. The model was run to steady state using a leapfrog timestepping with Asselin filter.

\subsection{Dry runs}

The presence of moisture modifies the temperature structure of the convecting atmosphere, and the fact that moisture is interactive complicates the issue further. Before we analyze the behavior of the moist model, we can explore the dry model in which moist convection is turned off. In this case $H_{e}$ is either the integral of the radiative equilibrium profile, or 


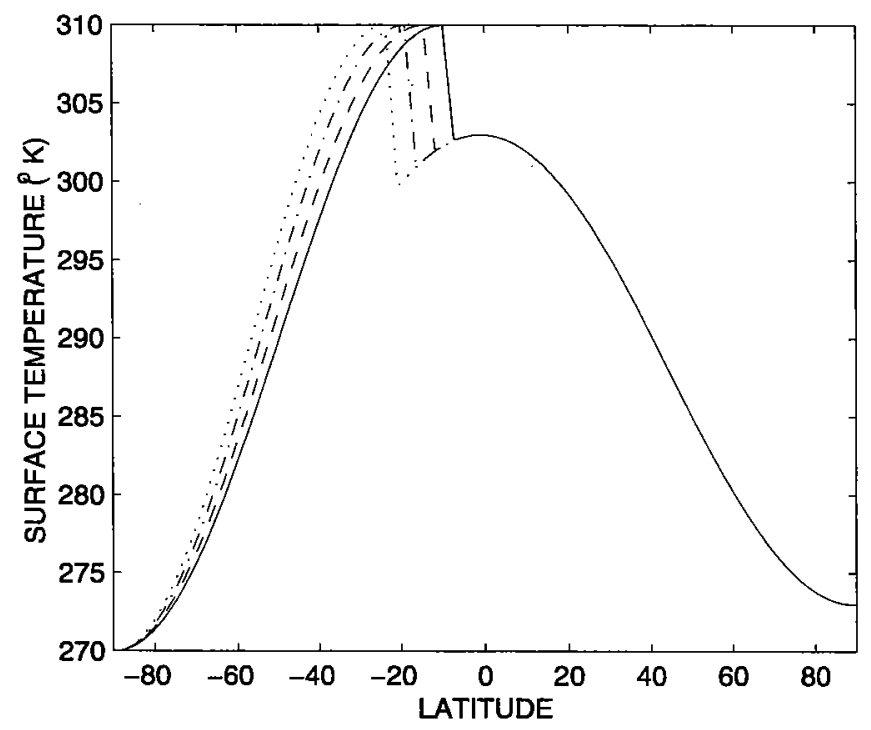

Figure 1: Surface temperature forcing. Two maxima are present: one representing the oceanic maximum and one the land. The maximum oceanic temperature is at the equator. The maximum land temperature is reached at $10^{\circ}, 15^{\circ}, 20^{\circ}, 25^{\circ}$ degrees latitude for the solid, dashed, dash-dotted, straight lines respectively.

the result of dry convection, which is only dependent on the potential temperature of the mixed layer and therefore on the imposed surface temperature.

Four profiles of surface temperature are shown in figure (1). The temperature profile exhibits two local maxima, one at the equator of about $30^{\circ} \mathrm{C}$, and one off the equator of $37^{\circ} \mathrm{C}$, which are chosen to represent the maximum oceanic temperature and the maximum land temperature respectively. There is no other distinction between land and ocean. Figure (2) shows the meridional profiles of zonal wind corresponding to the four cases of surface temperature. For all four the solution involves an angular momentum conserving meridional circulation, meaning that the meridional temperature gradient is sufficiently sharp to sustain a meridional circulation.

Two different behaviors are observed. For an off-equatorial heating sufficiently far from the equator, a local circulation is established. In these cases (bottom panel) the mass balance (29) is established within a few degrees of the forcing. Equatorward of that circulation an ordinary Hadley cell is established (the same case has been run without a temperature maximum at the equator and the same off equatorial circulation develops).

For simulations were the off-equatorial heating is closer to the equator, only one crossequatorial circulation develops and the Hadley cell disappears (figure (2), top panel).

Two of these cases are compared in figure (3). Sinks of mass, corresponding to where the free atmosphere is not convecting and therefore cooled towards radiative equilibrium, are clearly represented by regions where the restoring height is much smaller than the simulated height. In case 1 the model develops a strong meridional circulation centered on the maximum temperature of the equator. In case 4 the off equatorial maximum is sufficiently far from the equator that an independent Hadley cell develops. The different 

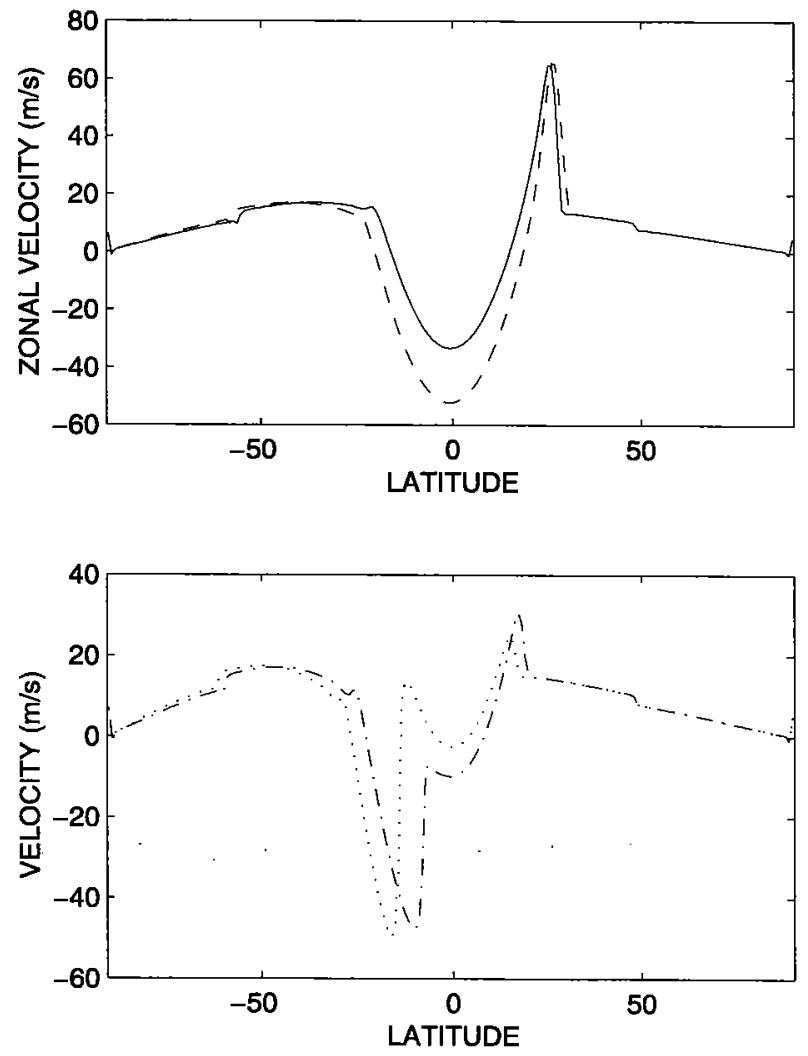

Figure 2: Zonal velocity. Top: cases 1 and 2 corresponding to a maximum continental temperature at $10^{\circ}$ (solid) and $15^{\circ}$ (dashed) latitude. Bottom: cases 3 and 4 corresponding to a maximum continental temperature at $20^{\circ}$ (dash-dotted) and $25^{\circ}$ (dotted) latitude. 
circulation is particularly evident in the meridional wind profiles (figure (3), bottom panel). For case 1 we can see one large meridional circulation whereas for case 4 two cells are present.

Without moisture it appears to be very difficult not to obtain a meridional circulation. Temperature contrasts of a few degrees, distributed over a distance of a few degrees latitude, representing the temperature contrasts observed at the onset of the Asian monsoon, seem to be sufficient to obtain a meridional circulation. As the maximum surface temperature is moved away from the equator though, the cross-equatorial circulation is lost to a more local one; we will focus only on cross-equatorial circulations as models of the Asian monsoon.

The presence of moisture and maybe convection over the ocean must be crucial in preventing the formation of a monsoon, when India is hot but dry, as happens in May [2] just before the onset of the monsoon itself.

\subsection{Moist runs}

To understand the effects of moisture we first seek a distribution of surface temperatures that in the absence of moist convection would not be able to trigger a monsoon. In order to obtain such equilibrium solution, an unrealistic temperature distribution is needed (figure (4)): the maximum land temperature is placed at $30^{\circ}$ latitude off the equator and the transition to oceanic temperatures occurs over $25^{\circ}$ latitude. When moisture is turned off, the flow is in substantial equilibrium with the thermal forcing (figure (5), top panel), and only a hemispheric Hadley Cell is present.

Two moist cases are now presented. In the first case moisture is forced over the ocean only. No moisture is present over land but the one brought in by advection by the mean flow. This simulation represents the state of the Indian region before the onset of the monsoon, with a hot and dry Indian continent and a warm ocean. Land starts at $5^{\circ} S$ latitude and spreads southward to the pole.

As shown in figures (5), middle panel, and (6), the effect of moisture overrides the temperature gradient. As soon as the mixed layer is moistened, the convective temperature profile makes the equivalent height of the atmosphere over the ocean much greater than that over land. A strong meridional circulation then develops, with rising air over the ocean and subsiding elsewhere, in particular over land. In this situation a monsoon develops opposite to the observed.

The region of convection is very narrow and positioned off the equator on the hemisphere opposite to were land is. The width of the convecting region is the result of the cooperation of advection of moisture and the drying effect of upper tropospheric convergent flow, which tend to concentrate moisture in a delta-form distribution.

In the absence of a triggering mechanism, initial conditions similar to the observed are insufficient to initiate the development of a monsoon. If on the other hand all that is missing is in fact a triggering mechanism, if we artificially bring in some moisture over land, then the circulation should be as the one observed and should be able to sustain itself.

We therefore change the initial conditions by imposing that land be initially moist. If during the the initial part of the simulation we also allow land to be a source of moisture, then all the domain is moist and the temperature gradient establishes the direction of the circulation. If the moisture source over land is subsequently turned off, so that the forcing 

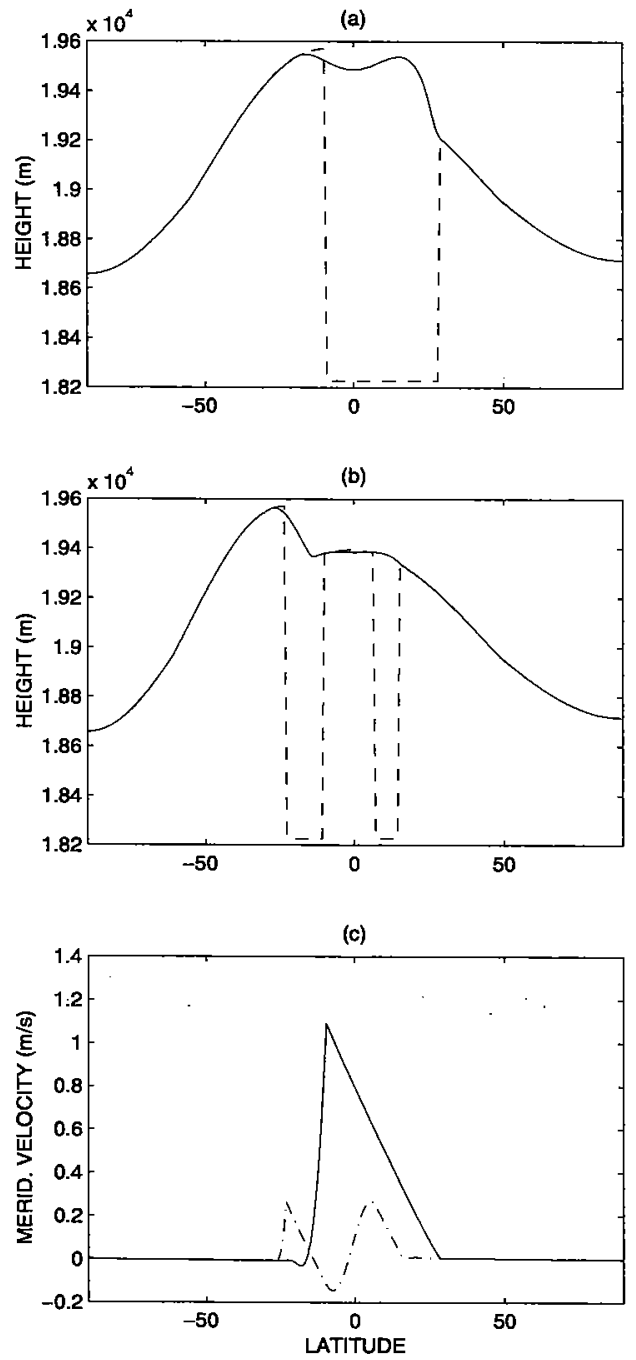

Figure 3: (a) Height (solid) and restoring height (dashed) for case 1 (10 max. temperature). (b) Same as (a) but for case $4\left(10^{\circ}\right.$ max. temperature). (c) Meridional velocity for case 1 (solid) and 4 (dashed). 


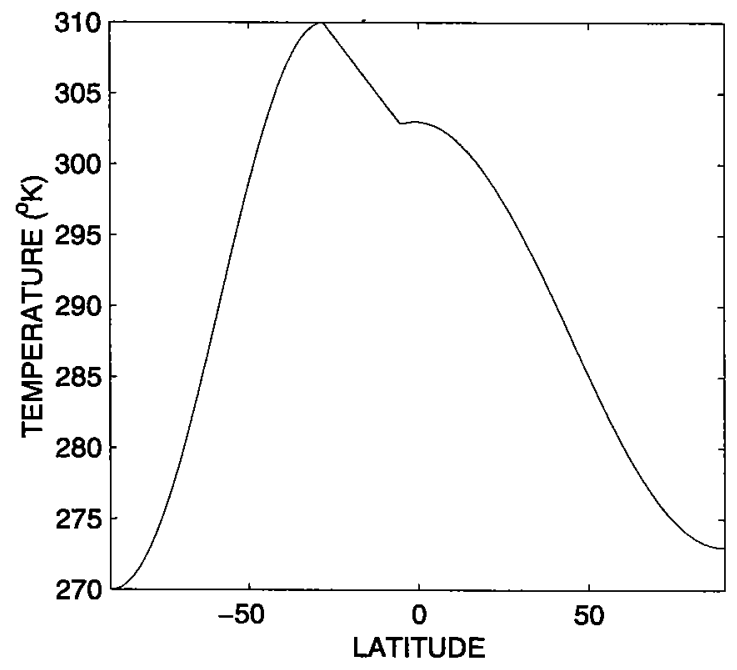

Figure 4: Temperature forcing for the moist experiment on Xie's arguments [2]

is now identical to the previous case, the circulation does not change and remains divergent over land (figure (5), bottom panel, figure (6)).

Once moisture is introduced over land, a circulation is established that is unable to dry out completely the mixed layer and draws in water from the ocean, which is now upwind, so that the circulation is able to sustain itself.

The ocean is, unrealistically, the only source of moisture in this model. Note that although heating is implied by the value of $h$, we have made no attempt to diagnose precipitation from that implied heating. Neglecting precipitation and subsequent evaporation from the ground is an important shortcoming of the model. Furthermore, drying of the mixed layer is accomplished through large scale convergence, whereas convective downdrafts, also diagnosed from the implied heating, would have been a better choice. These improvements are left for future work.

Despite these deficiencies, these simulations show that the same surface forcing with different initial conditions produces different solutions: the axisymmetric model exhibits multiple equilibria.

\section{Discussion and conclusions}

It has been argued [2] that a monsoon in a simplified geometry is the result of a zonally asymmetric disturbance that brings moisture over land. Before the disturbance propagates the wind profile is in thermal equilibrium with the surface forcing. The explosive nature of the monsoon is related to the abruptness of the instability that suddenly brings moisture over land, initiating moist convection.

An axially symmetric model was constructed to analyze these questions in a simplified setting. The formulation of this model differs from previous works in many ways: it is a model for the vertically integrated temperature profile and the upper tropospheric flow; it does not require the specification of a mean static stability; it contains a physically 

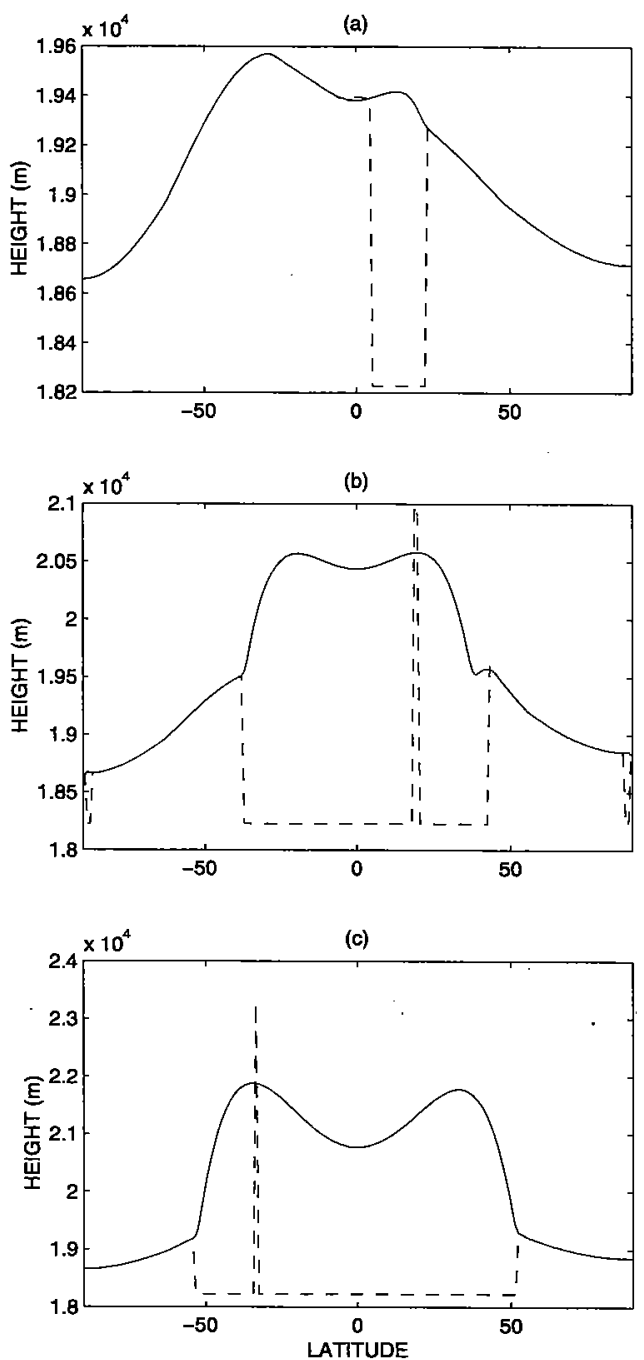

Figure 5: (a) Height and restoring height for the dry circulation. (b) Same as (a) but for moisture forced over ocean. (c) Same as (b) but with also initial moisture over the land added. Land starts where the temperature begins increasing poleward. 
(a)

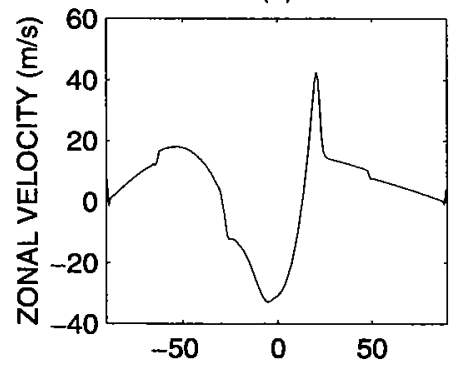

(d)

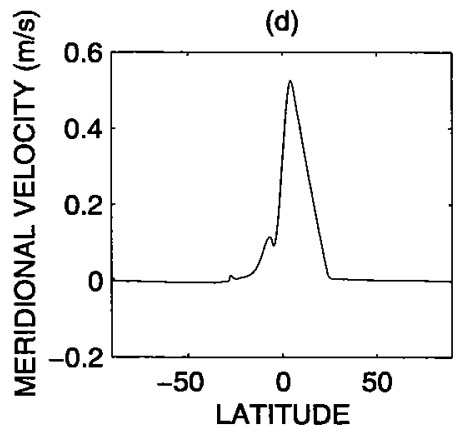

(b)

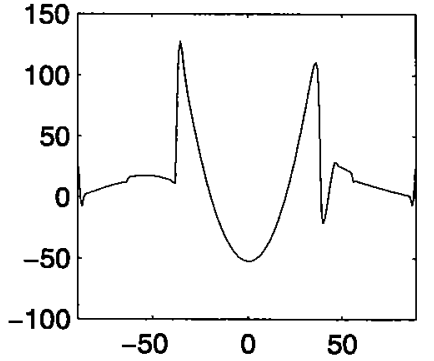

(e)

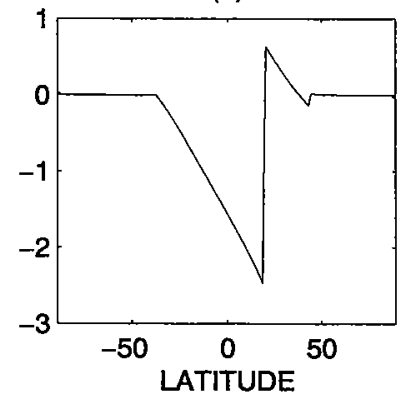

(c)

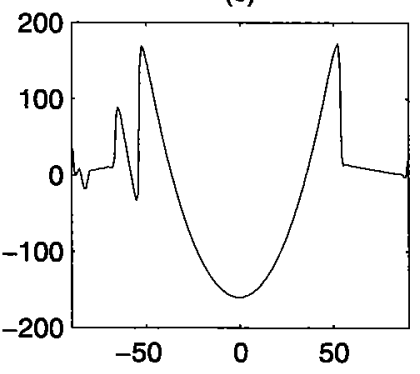

(f)

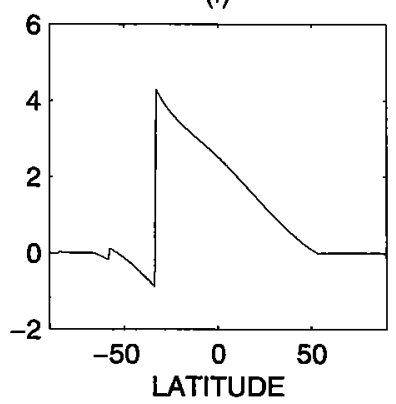

Figure 6: (a), (b), (c), zonal velocities for the dry case, the only ocean moist case and the moist land case respectively. (d), (e), (f) same as (a), (b), (c) but for meridional velocity.

motivated parameterization for moist convection.

In the absence of moist convection a thermal wind in balance with the forcing is hard to achieve unless the temperature contrasts are spread out over a few thousand kilometers. Starting from a state that is indeed in equilibrium without moist convection, we have shown that the final steady state solution is a function of the initial distribution of moisture.

Initializing the model with a dry and hot continent and a source of moisture over the ocean, as is the case for India before the onset of the monsoon, does not produce a monsoon, but rather a circulation with the same character but opposite in sign. If moisture is artificially introduced over land, then a monsoon like the observed develops. The model can therefore exhibit multiple equilibria.

The model does allow for the adjustment of static stability. We have seen in section (2.2) that there are two processes besides advection that can alter the temperature of the free atmosphere: convective processes and large scale convergence. In all experiments the convective closure is the dominant process heating (cooling in the case of non-convecting atmosphere) the free atmosphere. In all experiments, convergence in the presence of a positive static stability never grows to more than $5 \%$ of the convective closure. Furthermore, variations of static stability in this model are limited to no more than $15 \%$, making convergence a secondary process from a thermodynamical point of view.

More work is needed to establish the behavior of this model in a wider parameter range: multiple equilibria have been achieved using a highly unrealistic surface temperature profile. For very shallow temperature gradients, moisture is necessary to trigger any kind of off equatorial meridional circulation. We have seen though that more realistic surface 
temperature profiles are able to trigger a monsoon in the complete absence of moisture. It is possible that by introducing moisture over the ocean such temperature profiles would still exhibit multiple equilibria, but for sufficiently strong temperature gradients we expect this behavior to disappear. What is the behavior of the model between these two extreme cases needs to be investigated.

\section{Acknowledgements}

I would like to thank Alan Plumb for suggesting this project and helping throughout; Isaac Held for an inspiring set of lectures and many useful discussions; Wayne Schubert for his infinite patience and for the many enlightening discussions on convection; Rick Salmon, for organizing an unforgettable summer; all the staff at Walsh Cottage, for being such a remarkable group. Finally, I would like to thank all my friends, Karen, Cheryl, Zhiming, Chris W, Christos, Lucy, Chris S, Tivon, and of course the Pontiac Catalina. Thank you all.

\section{References}

[1] R. A. Plumb and A. Y. Hou, "The response of a zonally symmetric atmosphere to subtropical thermal forcing," J. Atmos. Sci. 49, 1790 (1992).

[2] S.-P. Xie and N. Saiki, "Abrupt onset and slow seasonal evolution of summer monsoon in an idealized gcm simulation," J. Met. Soc. Japan 77, 949 (1999).

[3] I. M. Held and A. Y. Hou, "Nonlinear axially symmetric circulations in a nearly inviscid atmosphere," J. Atmos. Sci. 37, 515 (1980).

[4] K. M. Xu and K. A. Emanuel, "Is the tropical atmosphere conditionally unstable?," Mon. Wea. Rev. 117, 1471 (1989). 


\title{
A Theory for the Quasi-biennial Oscillation Based on Gravity Wave Breaking and Saturation
}

\author{
Lucy Campbell
}

\begin{abstract}
This paper describes a model for the quasi-biennial oscillation based on the theory of gravity wave breaking and saturation. In the model, gravity waves are forced at low altitude and propagate vertically upwards, their amplitude increasing with height. In regions where the waves begin to overturn, dissipation is added locally, so that the waves are prevented from breaking. This results in momentum flux divergence and acceleration of the zonal mean velocity.
\end{abstract}

\section{Introduction}

The quasi-biennial oscillation (QBO) is an alternating pattern of westerly and easterly zonal mean winds observed in the equatorial stratosphere. The oscillation has an average period of 26 months and a maximum amplitude of about $20 \mathrm{~m} \mathrm{~s}^{-1}$ and is symmetric about the equator occurring between $12^{\circ} \mathrm{S}$ and $12^{\circ} \mathrm{N}$. In the past few decades, a number of theories have been developed to explain the QBO. It is well-known now that it is driven by wavemean flow interactions of waves propagating upwards from the troposphere. There has been some debate as to the type of waves that are involved, i.e., large scale Rossby-gravity waves and Kelvin waves and/or mesoscale internal gravity waves. It is now generally recognized that to generate a large enough vertical momentum flux to drive the $\mathrm{QBO}$, the gravity wave fluxes must be present. The review article of Dunkerton [1] discusses the role played by gravity waves in generating the QBO.

The QBO has been simulated quite successfully using one-dimensional models in which the waves interact with the mean flow but wave-wave interactions are ignored. Lindzen and Holton [2] initially postulated that the waves interact with the mean flow through absorption at a critical level where the mean velocity is equal to their phase speed. Oscillations in the mean velocity would result on specifying a suitable upper boundary condition to simulate an equatorial semi-annual oscillation (period $\sim 6$ months). However, a few years later, they re-evaluated this theory including the effects of wave dissipation due to Newtonian cooling [3]. The mechanism by which dissipation of the waves could produce an oscillation of the mean flow was presented explicitly by Plumb [4] using a numerical model and by the laboratory experiments of Plumb and McEwan [5]. One of the key features emerging from [4] is that the period of the oscillation is inversely proportional to the square of the amplitude of the forcing. 
In recent years, some GCMs (general circulation models) have been able to spontaneously produce QBOs (for example, [6], [7]). It is interesting to note that the majority of these oscillations have periods that quite closely approximate the period of the observed QBO. This is quite surprising given the fact that the GCMs all use different formulations, in particular, different gravity wave parameterizations, and hence they must certainly employ different forcing amplitudes (see, for example, [8], for a comparison of several GCMs, some of which are able to generate QBOs). According to the wave-mean flow interaction models [4], one would have expected their periods to differ greatly.

The motivation for this GFD Summer Project was to find an explanation for this. This report describes a simple model for the QBO that was developed in an attempt to better understand the relationship between the forcing amplitude and the period of the oscillation. The model is based on the idea of gravity wave breaking and saturation described by Lindzen [9]. The formulation of the model is similar to that of Plumb [4]. The main difference is that dissipation is introduced locally only when needed to prevent wave breaking. Because of the similarity with Plumb's model, a brief description of that model is given in section 3. In section 4, Lindzen's gravity wave saturation criterion is described. Results of the simulations are presented in section 5 .

\section{Generation of a QBO by dissipation}

In this section, a brief review is given of the mechanism by which a $\mathrm{QBO}$ can be generated by dissipation of waves. The details are given in [4]. Note that the notation here differs slightly from that of [4]; in particular, the streamfunction here has been defined as the negative of the one used there:

$$
\frac{\partial \psi}{\partial z}=-u, \quad \frac{\partial \psi}{\partial x}=w,
$$

so certain terms in the equations below are of opposite sign to the corresponding terms in $[4]$.

The equations for the streamfunction $\psi$ and the buoyancy $\sigma$ (defined in terms of the density $\rho$ as $-g \Delta \rho / \rho$ ) are

$$
\frac{\partial \nabla^{2} \psi}{\partial t}+\frac{\partial \sigma}{\partial x}-\nu \nabla^{4} \psi+J\left(\psi, \nabla^{2} \psi\right)=0
$$

and

$$
\frac{\partial \sigma}{\partial t}-N^{2} \frac{\partial \psi}{\partial x}+\mu \sigma+J(\psi, \sigma)=0
$$

For the moment, it is assumed that all the variables and parameters are dimensionless (see section 4 ). The equations are linearized about a mean state by writing $\psi(x, z, t)=$ $\bar{\psi}+\epsilon \psi^{\prime}(x, z, t)$ where $\bar{\psi}$ is the zonal mean and $\psi^{\prime}$ the disturbance streamfunction. The non-dimensional parameter $\epsilon$ then gives a measure of the magnitude of the perturbation quantities relative to the mean flow quantities. The computational domain is a rectangular region at the lower boundary of which a forcing of the form $\psi^{\prime}=e^{i k(x-c t)}$ is applied. The solution then takes the form

$$
\psi^{\prime}(x, z, t)=\phi(z) e^{i k(x-c t)}
$$


where the amplitude $\phi(z)$ of the disturbance satisfies the equation

$$
\frac{\partial^{2} \phi}{\partial z^{2}}+\left\{\frac{N^{2}[1+i \mu / k(\bar{u}-c)}{(\bar{u}-c)^{2}}-\frac{\bar{u}_{z z}}{\bar{u}-c}-k^{2}\right\} \phi=0 .
$$

Viscosity has been ignored in this last equation with the assumption that viscous dissipation of the waves is much less than thermal dissipation [4]. An equation for the evolution of the mean flow is obtained by averaging equation (1) over a wavelength $2 \pi / k$ :

$$
\frac{\partial \bar{u}}{\partial t}-\nu \frac{\partial^{2} \bar{u}}{\partial z^{2}}=-\epsilon^{2} \frac{\partial}{\partial z}\left(\overline{u^{\prime} w^{\prime}}\right)
$$

The numerical model comprises equations (3) and (4). To generate a QBO with this model, there must be two waves with phase speeds of opposite sign, i.e., $e^{i k_{1}\left(x-c_{1} t\right)}, e^{i k_{2}\left(x-c_{2} t\right)}$. We shall assume that $c_{2}=-c_{1}$. It is assumed that $\bar{u}$ is slowly varying and $\bar{u}_{z z} \approx 0$ and that $k^{2}$ is sufficiently small that, equation (3) can be solved using the WKB method (see, for example, Bender and Orszag [10]) and the momentum flux for each wave calculated. These are shown to be:

$$
F_{n}(z)=F_{n}(0) \exp \left\{-\int_{0}^{z} \frac{N \mu}{k(\bar{u}-c)^{2}} d z^{\prime}\right\} \quad n=1,2 .
$$

Note that, in the absence of dissipation, the momentum fluxes would be independent of height as required by the Eliassen-Palm non-acceleration theorem. The right hand side of equation (4) is the sum of the gradients of the momentum fluxes for the 2 waves. It was shown in [4] that the sign of $\overline{u^{\prime} w^{\prime}}$ is the same of the sign of the phase speed of the waves. Thus the wave with positive phase speed accelerates the mean velocity while the one with negative phase speed decelerates it.

\section{Gravity wave breaking}

Following Lindzen [9], but using the notation of section 2 (with $\mu=0$ ), the criterion for wave breaking can be determined as follows. First, note that, with $\mu=0$, the equation for the amplitude of the perturbation streamfunction can be written as

$$
\frac{\partial^{2} \phi}{\partial z^{2}}+\lambda^{2}(z) \phi=0
$$

where

$$
\lambda^{2}(z)=\frac{\mathrm{N}^{2}}{(\bar{u}-c)^{2}}-\frac{\bar{u}_{z z}}{\bar{u}-c}-k^{2} .
$$

If $\bar{u}$ is slowly varying and $\bar{u}_{z z} \approx 0$, then an approximate solution can be found for $\phi$ using the WKB method:

$$
\phi \sim \lambda^{-1 / 2} e^{i \int \lambda d z} .
$$

Now the density perturbation is given by

$$
\rho^{\prime}=-\frac{\bar{\rho}_{z}}{\bar{u}-c} \phi e^{i k(x-c t)}
$$


The condition for wave breaking is that the density gradient be positive, i.e.,

$$
\epsilon\left|\frac{\partial \hat{\rho}}{\partial z}\right| \geq\left|\bar{\rho}_{z}\right|
$$

To leading order,

$$
\frac{\partial \hat{\rho}}{\partial z} \sim-\frac{\bar{\rho}_{z}}{\bar{u}-c} \lambda^{1 / 2} e^{i \int \lambda d z}
$$

so the waves break if

$$
\frac{\epsilon\left|\lambda^{1 / 2}\right|}{|\bar{u}-c|} \geq 1
$$

This is approximately equivalent to saying

$$
\frac{\epsilon\left|N^{1 / 2}\right|}{(\bar{u}-c)^{3 / 2}} \geq 1
$$

Thus, the waves are more likely to break if $N$ is large or if $\bar{u}-c$ is small.

Consider now the case where $\mu$, the diffusion coefficient in equation(3), is non-zero. Then

$$
\lambda^{2} \approx \frac{\mathrm{N}^{2}}{(\bar{u}-c)^{2}}\left(1+\frac{i \mu}{k(\bar{u}-c)}\right)
$$

and $\lambda$ has an imaginary part. The detailed analysis of Lindzen [9] showed that the condition under which the dissipation would prevent the growth of $\left|\frac{\partial \rho}{\partial z}\right|$ is that the imaginary part of $\lambda$ satisfies

$$
\left|\lambda_{I}\right| \approx \frac{\partial}{\partial z}\left(\frac{\epsilon\left|N^{1 / 2}\right|}{(\bar{u}-c)^{3 / 2}}\right) .
$$

\section{Numerical model and results}

The model equations for our simulation are, as given in section 2 ,

$$
\frac{\partial^{2} \phi}{\partial z^{2}}+\left\{\frac{N^{2}[1+i \mu / k(\bar{u}-c)}{(\bar{u}-c)^{2}}-\frac{\bar{u}_{z z}}{\bar{u}-c}-\alpha k^{2}\right\} \phi=0
$$

for the amplitude of the perturbation streamfunction and

$$
\frac{\partial \bar{u}}{\partial t}-\nu \frac{\partial^{2} \bar{u}}{\partial z^{2}}=-\epsilon^{2} \frac{\partial}{\partial z}\left(\overline{u^{\prime} w^{\prime}}\right)
$$

for the time evolution of the zonal mean wind. The various quantities have been nondimensionalized with respect to reference values as summarized in the table below. The range of values used in the simulations for each of the non-dimensional quantities is given as well as approximate values for some of the dimensional quantities. Asterisks (*) denote dimensional quantities. 


\begin{tabular}{|c|c|c|}
\hline Non-dimensional & Dimensional & Reference quantities \\
\hline $\bar{u}^{*}$ & $\bar{u} \approx 0.1$ & $U$ \\
\hline$c^{*} \sim 10-20 \mathrm{~m} \mathrm{~s}^{-1}$ & $c= \pm 0.2$ & $U \sim 50-100 \mathrm{~m} \mathrm{~s}^{-1}$ \\
\hline$t^{*}$ & $t$ & $T$ \\
\hline$z^{*} \leq 50 \mathrm{~km}$ & $z \leq 10$ & $H \sim 5 \mathrm{~km}$ \\
\hline$x^{*}$ & $x \leq 2 \pi$ & $L$ \\
\hline$\phi^{*}$ & $\phi$ & $\varphi$ \\
\hline
\end{tabular}

Table 1: Reference quantities used in the non-dimensionalization.

The non-dimensional parameter $\epsilon=\varphi /(L U)$ gives a measure of the amplitude of the perturbation at the forced boundary and $\alpha=H / L$ is the aspect ratio. The buoyancy frequency and hence the time scale $T$ of the evolution of the disturbance and the mean flow are set by the choice of vertical scale $H$, the velocity scale $U$ and the aspect ratio. The vertical scale is taken to be much smaller than the horizontal scale, so that $\alpha<<1$.

The equations were solved using standard second-order finite-difference approximations for the $z$-derivatives. The computational domain was the rectangular region $0<x<2 \pi$, $0<z<h=10$. The initial mean velocity took the form of a jet centered at $z=5$ : $\bar{u}(z, 0)=b \operatorname{sech}^{2}(z-5)$, where $b$ is a positive constant. Two waves of the form $e^{i k_{n}\left(x-c_{n} t\right)}$, with $k_{1}=1, c_{1}=0.2$ and $k_{2}=1, c_{2}=-0.2$, were forced at $z=0$ and propagated up to interact with the jet. The constant $b$ was chosen to be less than 0.2 , so that the Dopplershifted phase speed $(\bar{u}-c)$ of both waves was non-zero everywhere. In other words, there was no critical level for either wave initially and, consequently, none could be generated at later times ([4]). At the upper boundary of the computational domain, a radiation condition was applied to prevent reflections at the boundary. The buoyancy frequency $N$ was allowed to be dependent on $z$. It was chosen to satisfy

$$
N^{2}=N_{0}^{2} e^{2 z / h}
$$

where $N_{0}$ is a constant. With this choice of $N^{2}$, the perturbation streamfunction varies like $e^{z / 2 h}$. The WKB-defined vertical wavenumber is approximately $N /(\bar{u}-c)$. With $c= \pm 0.2$, $N \sim O(1)$ and $h=10$, there are then several vertical wavelengths within the computational domain.

The first few experiments were carried out with non-zero constant dissipation to reproduce the results of Plumb [4]. A QBO was generated with a period that depended on $\epsilon$ the amplitude of the forcing. Figure 1 shows time-height plots of the zonal mean velocity computed using this model. The set of parameters used in both graphs was as follows: $N_{0}=2.0, \mu=10^{-3}, \nu=5 \times 10^{-4}, \alpha=2 \times 10^{-3}$. Initially the mean velocity profile took the form of a positive jet in the region $4<z<6$ and approxiamtely zero elsewhere. In Figure $1(\mathrm{a})$, this positive region has descended to $z=0$ by $t \approx 2700$. This positive phase is followed by a negative phase (approximately $2700<t<5000$ ) and then another positive phase, and so on. The period of the oscillation appears to be about 5500 non-dimensional time units. This corresponds to a dimensional period of magnitude of about a month using the reference values given in Table 1 . By decreasing $\epsilon$ from 0.1 to 0.05 , the period of the 
oscillation was increased as predicted by the theory described in section 2 . With a still smaller forcing amplitude, a more realistic period could be obtained.

Having verified that the model could produce an oscillation when set up in this way with constant dissipation throughout the computational domain, we set about implementing the gravity wave saturation criterion described in section 3 . With our choice of $N^{2}$, the criterion (11) becomes

$$
\left|\lambda_{I}\right| \approx \frac{1}{2 h}-\frac{3}{2}\left(\frac{1}{\bar{u}-c} \frac{\partial \bar{u}}{\partial z}\right)
$$

The imaginary part of $\lambda$ is known from the expression for $\lambda$ and, from this, the dissipation coefficient $\mu$ required to prevent the waves from breaking may be evaluated. Clearly this $\mu$ depends on $z$; it is largest in regions where the waves are on the verge of breaking and small or zero elsewhere. The saturation technique can thus be summarized as follows. Initially $\mu$ is set to zero and the model run is started. Without dissipation, the momentum fluxes for the waves are independent of height and thus the only changes in the mean velocity result from the presence of the small but non-zero viscous term. The amplitude of the waves increases with height because of the exponentially increasing buoyancy frequency. Dissipation is introduced locally using (14) to prevent the waves from breaking. Thus, more dissipation is needed at higher altitudes and in particular near the center of the jet where $(\bar{u}-c)$ is smallest, but almost none near the ground. With such a set-up, the extent to which the mean velocity is accelerated should, to some extent, be insensitive to what occurs at low altitudes. It is conceivable then that the time scale of the evolution of the mean velocity would be independent of changes in the amplitude of the forcing.

The time-height plots resulting from this type of simulation are shown in Figure 2 for $\epsilon=0.1$ and $\epsilon=0.05$. Descending westerly and easterly phases are seen at higher altitudes; however the time scale of the descent is quite long and due to computational restrictions, the model has not yet been run for a long enough time to produce an oscillation. It is interesting to note that this time scale is almost the same for the two values of $\epsilon$. It remains to be seen whether, if the simulations are continued to the point where an QBO-like oscillation results, the period of the oscillation would indeed be the same for the two values of $\epsilon$. 
(a)

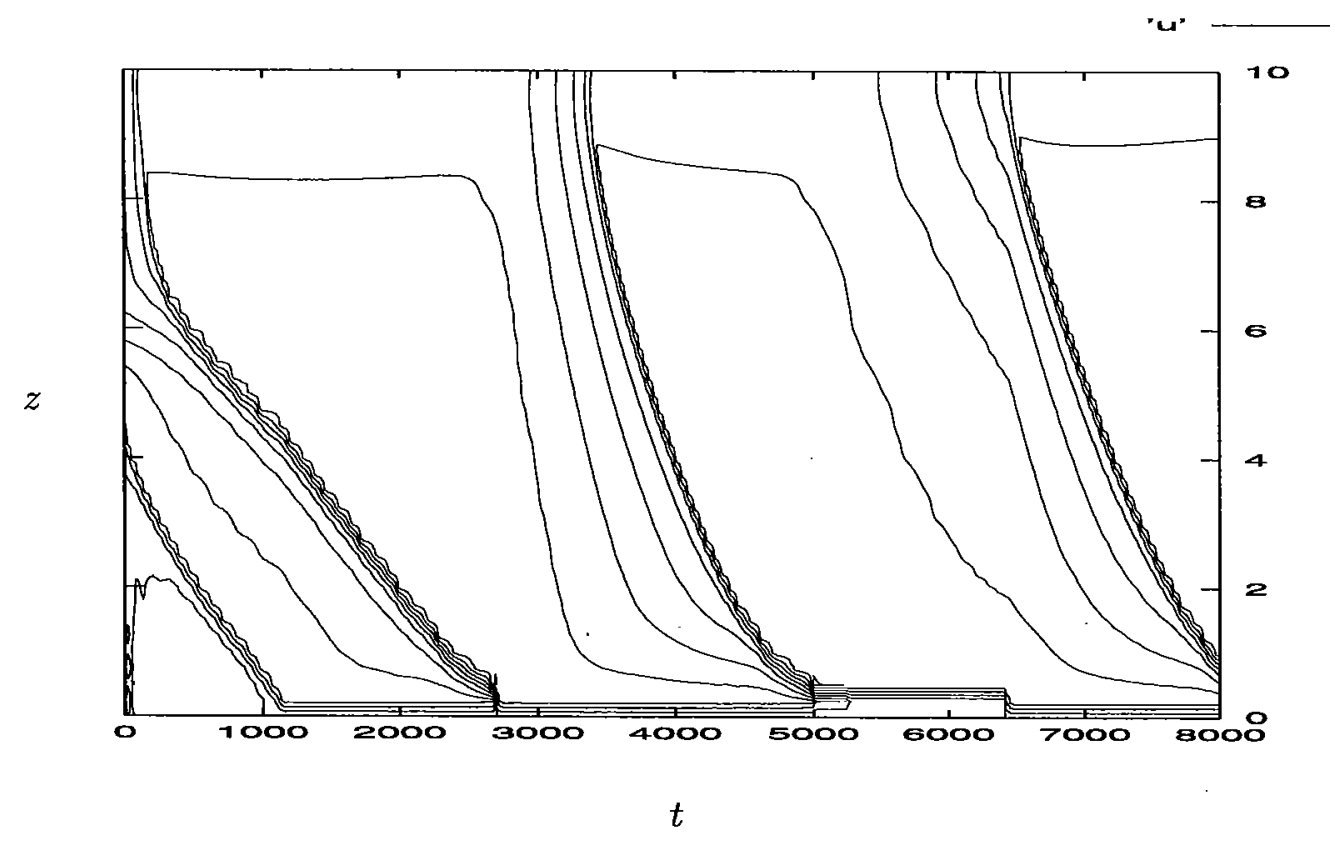

(b)

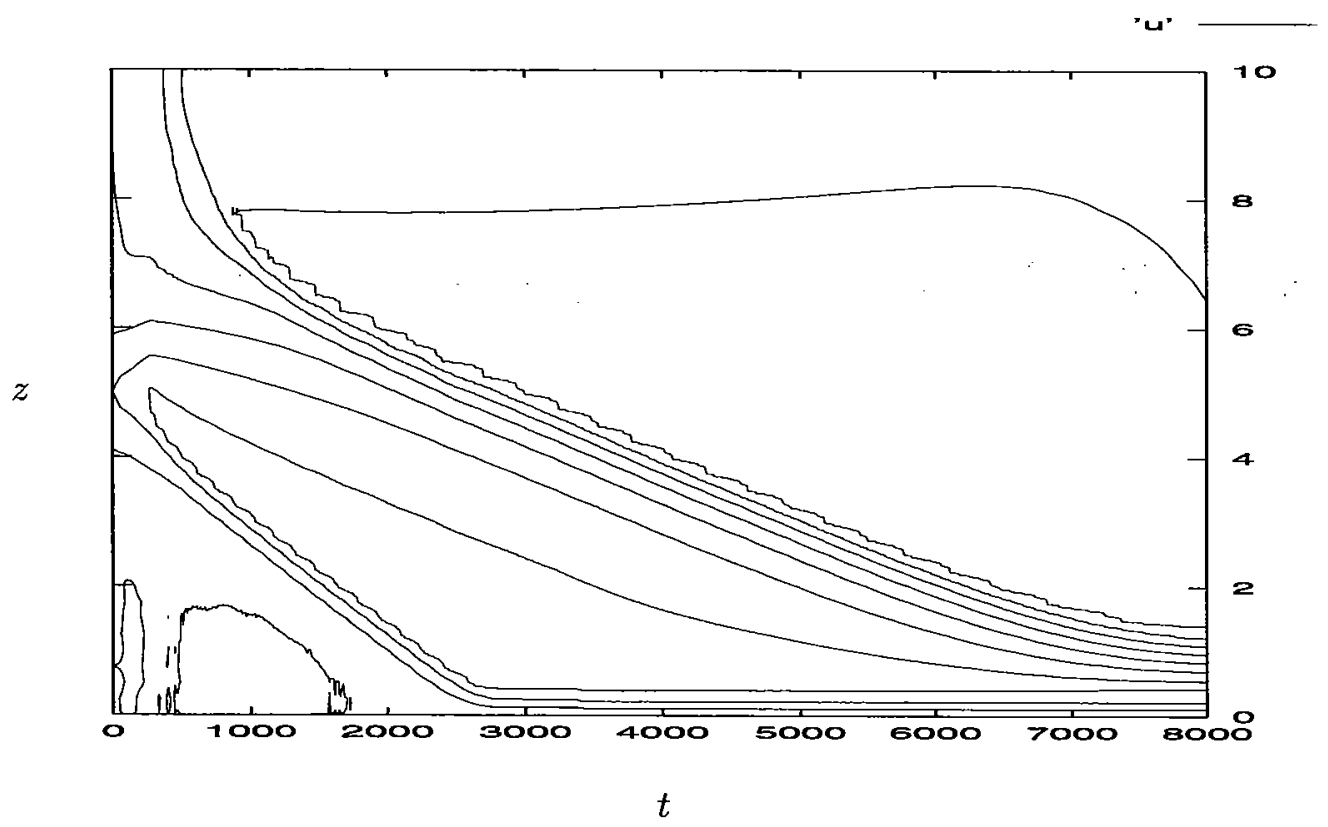

Figure 1: Time-height plots of the zonal mean velocity for the case of constant dissipation with (a) $\epsilon=0.1$ and (b) $\epsilon=0.05$ 
(a)

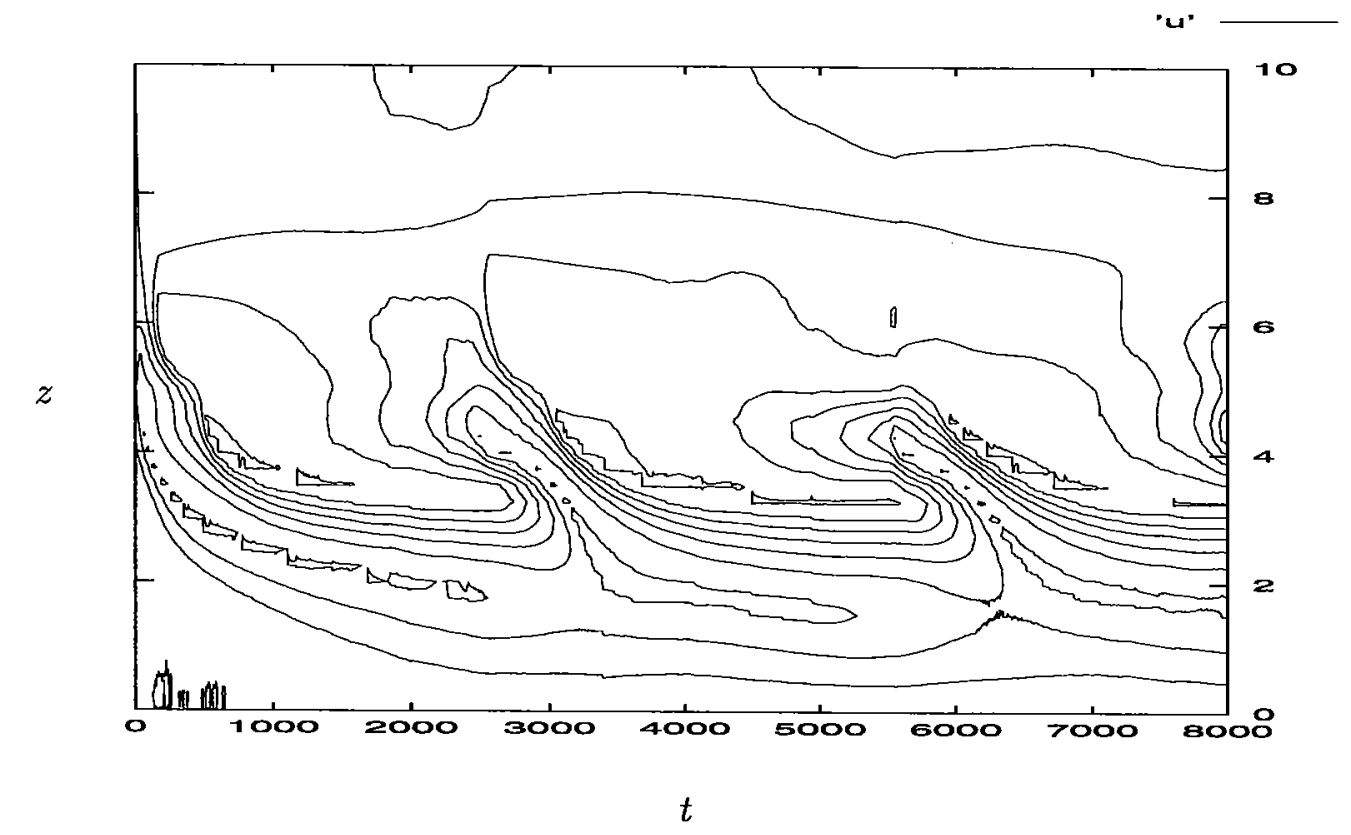

(b)

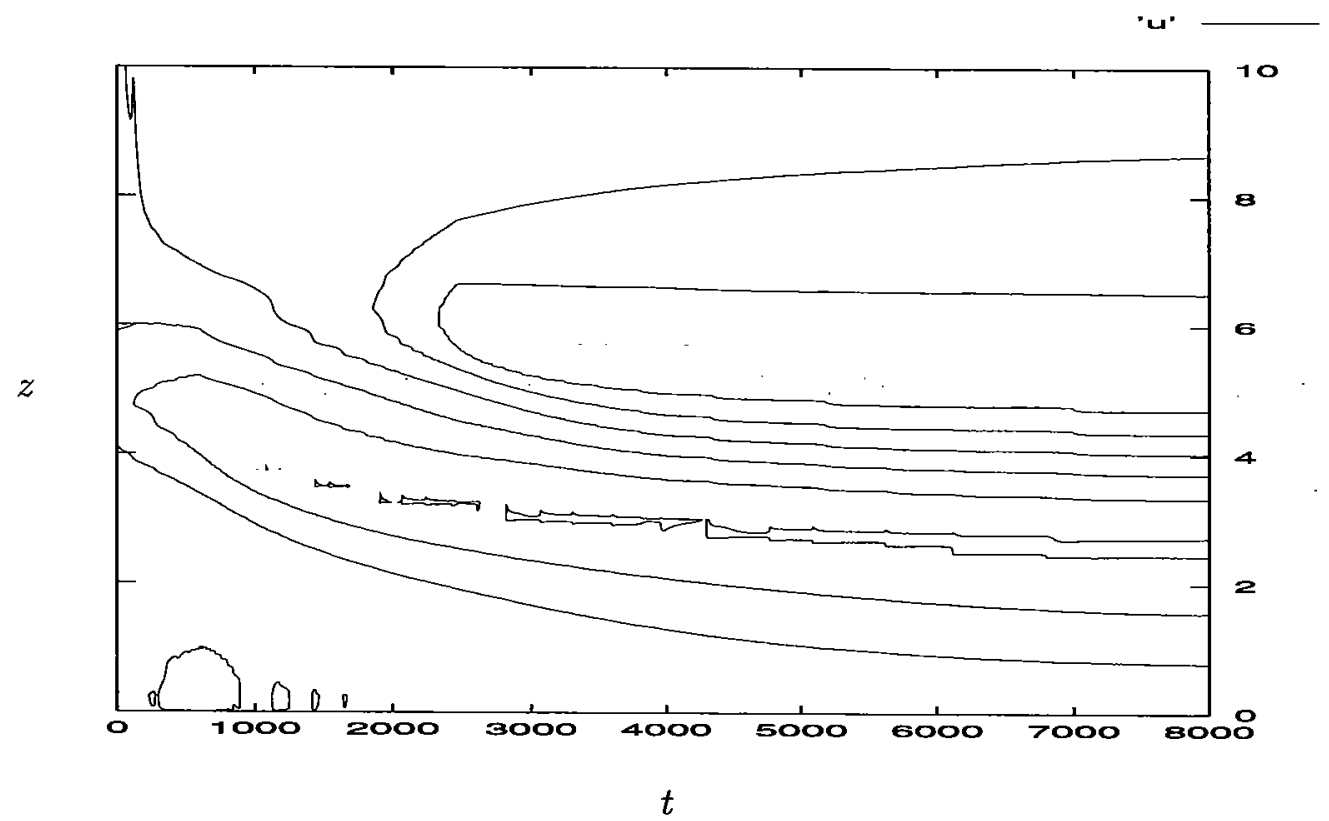

Figure 2: Time-height plot of zonal mean velocity with (a) $\epsilon=0.05$ and (b) $\epsilon=0.1$. Here, dissipation has been added to prevent the waves from breaking. 


\section{Conclusions}

This report described a model for the QBO in which the waves are dissipated locally in regions where their amplitudes grow to the point of breaking. By adding dissipation to the model in this way, the mean flow is accelerated/decelerated locally and descending westerly and easterly phases of the zonal mean velocity result. The time-scale on which this occurs is apparently insensitive to changes in the forcing amplitude.

In section 1, it was mentioned that most GCMs that produce QBOs get the period approximately right although they use different wave parameterizations. This suggests that the period of the QBO must be independent of the amplitude of the waves at the forcing level. It is possible then that, in reality, the QBO is generated by a mechanism such as that described in this report. However, this report describes work in progress. At the time of writing, only a limited number of runs had been carried out. A more extensive series of simulations with a wider range of choices of the relevant parameters and a longer total computational time would certainly shed more light on the the relation between the period and the amplitude of the forced waves. Ultimately, a full non-Boussinesq model may be required for a complete analysis of the problem. Also it would probably be necessary to include the effects of the higher harmonics, i.e., wave-wave interactions, as well as wavemean flow interactions.

\section{Acknowledgements}

I would like to thank Alan Plumb for suggesting this project to me and for his advice and guidance. I am also very grateful to Oliver Bühler and Isaac Held for very helpful criticism and suggestions and to the rest of the GFD 2000 fellows for their friendship during the summer. Finally I must say thanks to Rick Salmon for organizing such a great summer school.

\section{References}

[1] T. G. Dunkerton, "The role of gravity waves in the quasi-biennial oscillation," J. Geophys. Res. 102, 26,053 (1997).

[2] R. S. Lindzen and J. R. Holton, "A theory of the quasi-biennial oscillation," J. Atmos. Sci. 25, 1095 (1968).

[3] J. R. Holton and R. S. Lindzen, "An updated theory for the quasi-biennial cycle of the tropical stratosphere," J. Atmos. Sci. 29, 1076 (1972).

[4] R. A. Plumb, "The interaction of two internal waves with the mean flow: Implications for the theory of the quasi-biennial oscillation," J. Atmos. Sci. 34, 1847 (1977). 
[5] R. A. Plumb and A. D. McEwan, "The instability of a forced standing wave in a viscous stratified fluid: A laboratory analog of the quasi-biennial oscillation," J. Atmos. Sci. 35, 1827 (1978).

[6] R. J. W. K. Hamilton and R. S. Hemler, "Middle atmosphere simulated with high vertical and horizontal resolution versions of a GCM: Improvement in the cold pole bias and generation of a QBO-like oscillation in the tropics," J. Atmos. Sci. 56, 3829 (1999).

[7] T. Horinouchi and S. Yoden, "Wave-mean flow interaction associated with a QBO-like oscillation simulated in a simple GCM," J. Atmos. Sci. 55, 502 (1998).

[8] S. Pawson et al., "The GCM-reality intercomparison project for SPARC (GRIPS: Scientific issues and initial results," Bull. Am. Met. Soc. 81, 781 (2000).

[9] R. S. Lindzen, "Turbulence and stress owing to gravity wave and tidal breakdown," J. Geophys. Res. 86, 9707 (1981).

[10] C. M. Bender and S. A. Orszag, Advanced mathematical methods for scientists and engineers (McGraw-Hill, New York, 1978). 


\title{
Vorticity on a Barred Beach
}

\author{
Tivon Jacobson
}

\section{Introduction}

Longshore current modeling has been dominated by a radiation-stress viewpoint applied to shallow-water models. While the shallow-water model is arguably appropriate for gently sloping beaches, radiation-stress convergences are difficult to apply directly in the momentum equations when longshore ( $y$-coordinate) inhomogeneities are present. To date, longshore currents have been modelled with $y$-independent wave forcing. Should longshore inhomogeneities be fundamental to surf zone dynamics, the traditional models are insufficient. This has been pointed out in articles by D.H. Peregrine ([1], [2]), and was brought to our attention by F. Feddersen. [3]

Feddersen mentioned the problem of longshore currents on a barred beach. (Figure 1) There, waves breaking over bar and beach have their radiation stress convergences in these locations. The $y$-independent theory predicts the development of longshore currents over the bar and on the beach, a forecast at variance with observation. Experiments on a barred beach in North Carolina found the longshore current in the trough between bar and beach. [4] Laboratory experiments (and our numerical experiments of section 6) find that with wave forcing which does not depend on $y$, the longshore currents indeed do develop over bar and beach. Inhomogeneous forcing is a candidate to explain the observations.

Some theoretical difficulties with $y$-dependence may be overcome by considering vorticity forcing rather than radiation stress convergence. The vorticity sources are those due to wave breaking. With a sense of the location and strength of these vorticity sources, the problem becomes one of predicting the vortex dynamics which result. A first approximation for the location of the vorticity sources can be had using WKB theory and a parameterization of breaking. As well, Peregrine has derived a strength for such vortex sources using the circulation integral. [1] We found the Generalized Lagrangian Mean theory useful in

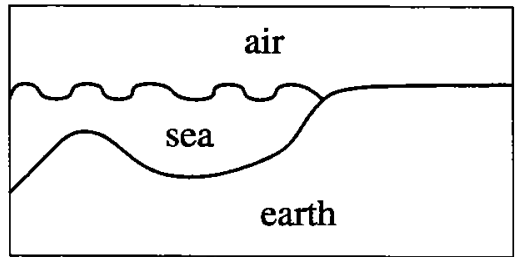

Figure 1: A Barred Beach. 


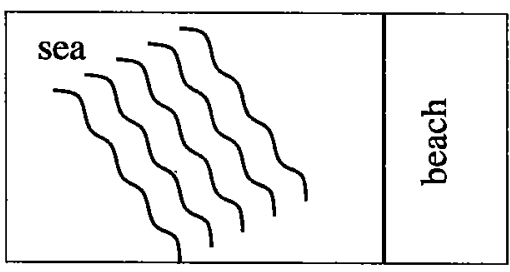

Figure 2: A wavetrain incident at an angle.

determination of these strengths, and discuss this in section 4. Some simple results which are helpful in predicting vortex dynamics are presented in section 5 .

A numerical model of the surf zone was used to resolve wave and vorticity alike. The model was a $2 \mathrm{D}$, finite-volume, shock-resolving shallow water model with varying bottom topography. The use of such a model to simulate vortex dynamics was expensive in computation time, since the gravity wave speeds provide a severe CFL restriction on the timestepping. Nevertheless, this model reperesents a means by which the entire wave-forcing process may be represented without parameterization.

The wave-forcing was considered to be by a wavetrain with longshore envelope incident on the beach at an angle. (Figure 2) We found that simple vortex dynamics predict a longshore current in the trough. This prediction was confirmed with the numerical model.

\section{Equations of Motion in Shallow Waters}

We will use a shallow water model for the surfzone throughout this article. Here we present the shallow-water equations for reference. Figure 3 shows the meaning of the variables.

$$
\begin{aligned}
u_{, t}+u u_{, x}+v u_{, y} & =-c_{00}^{2}\left(h_{, x}+h_{b, x}\right) \\
v_{, t}+u v_{, x}+v v_{, y} & =-c_{00}^{2}\left(h_{, y}+h_{b, y}\right) \\
h_{, t}+(h u)_{, x}+(h v)_{, y} & =0 .
\end{aligned}
$$

The variable $x$ represents cross-shore distances, the variable $y$ alongshore distances. This is a nondimensionalized model with the gravity $g$ and constant reference depth $H$ factored into $c_{00}=\sqrt{g H}$. No apologies are made for the use of the shallow-water model, although we note several modeling limitations in section 6 . While the use of shallow-water bores as a model of ocean-wave breaking lacks in its description of the breaking wave region itself, the location and rough magnitude of vorticity sources due to breaking are of more interest in this article. Moreover, precise models of wave-breaking may be desirable, but a 3D freesurface simulation of the surf zone is well beyond analytical or computational means. It is not beyond experimental means: wavetanks capable of such simulations exist. [5]

Our shallow-water model has no explicit viscous contribution. Viscous momentum mixing and bottom drag necessarily enter the longshore current picture in the forced-dissipative mean-state. We limit our concern to the initial transferral of wave-induced momentum change to the trough region. However the addition of bottom friction to the model is straightforward and we intend to carry this out in future work. 


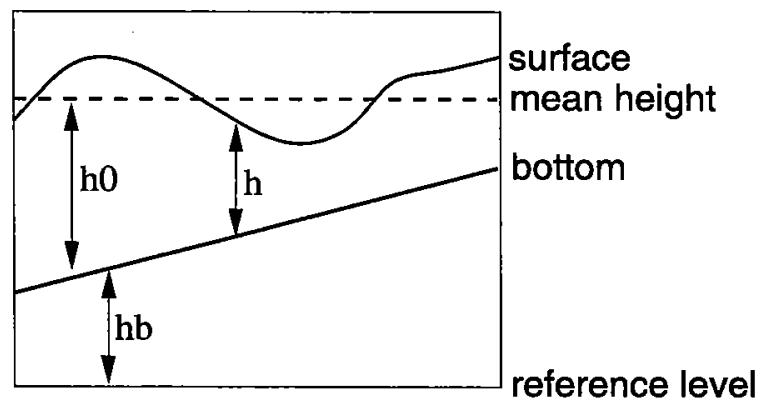

Figure 3: The Shallow Water Model.

\section{$3 \quad$ Ray Tracing}

Ray-tracing provides for approximate calculation of the wave amplitudes. These may be used in estimating vorticity source terms. We develop some ray-trace formulae and apply them as needed. A full development of these methods can be found in many places, such as [6]. An example of the use of this method to calculate vorticity sources may be found in [7]. Throughout, we assume that the mean flow has no effect on the waves. Generalizations of the method exist to deal with such a case, but the situation then becomes much more complex.

For frequency $\omega$ and wavenumber vector $\vec{k}$, we have:

$$
\begin{aligned}
\vec{k}_{, t}+\overrightarrow{c_{g}} \cdot \nabla_{x} \vec{k} & =-\nabla_{x} \omega \\
\omega_{, t}+\overrightarrow{c_{g}} \cdot \nabla_{x} \omega & =-\omega_{, t} .
\end{aligned}
$$

Here, $\omega=\omega(\vec{k}, \vec{x}, t)$ is the frequency. The dependence of $\omega$ on the wavenumber vector, the position and the time constitutes the dispersion relation. To find a law for the amplitude of the waves, one can take as starting point the conservation of wave-action ([8]) to arrive at:

$$
\left(\frac{h_{0} E}{\omega}\right)_{, t}+\nabla_{x} \cdot\left(\overrightarrow{c_{g}} \frac{h_{0} E}{\omega}\right)=-D\left(h_{0}, E\right) .
$$

The quantity $E$ is the wave energy density per unit mass. The function $D\left(h_{0}, E\right)$ is a dissipation which represents the effect of loss of wave energy due to breaking. For shallow water,

$$
\begin{gathered}
\omega(\vec{k}, \vec{x}, t)=c_{00} \sqrt{h_{0}(\vec{x})}|\vec{k}|, \\
E=\frac{1}{2}\left(|u|^{2}+c_{00}^{2} \frac{(\Delta h)^{2}}{h_{0}(\vec{x})}\right) .
\end{gathered}
$$

The quantity $\Delta h$ is the surface perturbation $\left(h-h_{0}\right)$. (See Figure 3) For linear monochromatic waves, we could replace $\Delta h$ by a harmonically varying wave height $h^{\prime}$. The polarization realtions for the linear wave then give 


$$
E=\frac{c_{00}^{2}}{h_{0}} \overline{h^{\prime 2}} .
$$

In our case there will be no topographic dependence on $y$. The dispersion relation is time-independent. As a consequence, equations 4 reduce to:

$$
\begin{aligned}
k_{, t}+c_{g x} k_{, x} & =-\omega_{, x} \\
l & =l_{0}, \text { constant along rays } \\
\omega & =\omega_{0}, \text { constant along rays } \\
\omega_{0} & =c_{00} \sqrt{h_{0}(x)} \sqrt{k^{2}+l_{0}^{2}} .
\end{aligned}
$$

The last three are sufficient to find the trajectories of rays, since rays are the integral curves of the group velocity, a function of $k, l, \omega$. The integration of the wave energy density by equation 6 can now be contemplated if the field of group velocities is known. From the field of wave energy density will come the vorticity source terms (Section 4).

Calculation of the wave energy density must take breaking into account. Consider the case in which the waves have reached a forced-dissipative equilibrium. We also assume independence of $y$ for expository purposes, so that the average square linear wave amplitude $\overline{h^{\prime 2}}$ in given (via equation 6 and 9 ) in terms of the bottom topography:

$$
\left(\sqrt{h_{0}} \overline{h^{\prime 2}}\right)_{, x}=-\frac{\omega_{0}}{c_{00}^{3}} D\left(E, h_{0}\right) \text {. }
$$

Assuming that the waves break only when the nonlinear amplitides are large enough, $\left(\sqrt{h_{0}} \overline{h^{\prime 2}}\right)_{, x}=0$ in non-breaking regions. The assumption is ad-hoc, but captures that we wish to see sudden transitions to wave-breaking over topographic features. One could introduce an additional law for the scaling of wave amplitude-in breaking regions and integrate equation 14 to get the energy density or mean-square amplitude field.

Figure 4 shows the cross-shore wave amplitudes (without breaking) in the case in which it is assumed that the waves have no alongshore envelope.

\section{Vorticity Sources, GLM, and the Circulation Theorem}

A presentation of Generalized Lagrangian Mean (GLM) theory may be found in the two papers by Andrews and McIntyre ([8], [9]). Some additional information about the theory, as well as examples of its application to the theory of breaking waves are in [10], [7]. Of essential interest to us is that GLM gives an approximate expession for the time rate of change of mean vorticity in terms of the evolution of the wave pseudomomentum. We have since found that the same results are available from the curl of the radiation-stress tensor expressed in terms of the infinitesimal waves. The exact finite amplitude equations are difficult to interpret in the present case, but rough estimates for sources in the breaking region in terms of infinitesimal wave quantities are available [7]:

$$
(\nabla \times \bar{u})_{, t}=\nabla \times \overrightarrow{\mathcal{F}}
$$




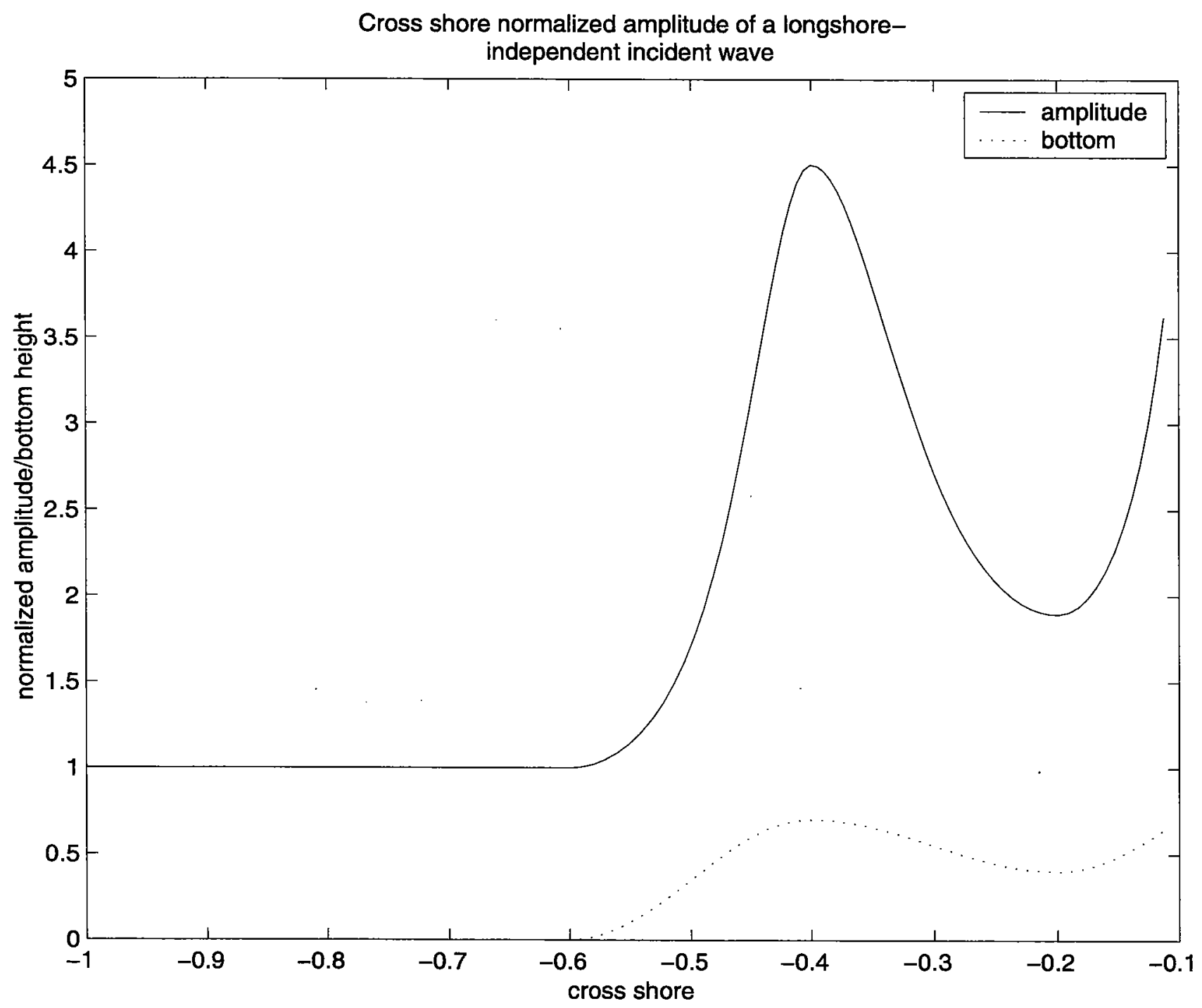

Figure 4: Example of Cross-Shore Amplitudes. 


$$
\begin{aligned}
& \mathcal{F}_{x}=\frac{k}{h_{0}}\left(h_{0} \frac{k}{\kappa^{2}} E\right)_{, x}+\frac{k}{h_{0}}\left(h_{0} \frac{l}{\kappa^{2}} E\right)_{, y} \\
& \mathcal{F}_{y}=\frac{l}{h_{0}}\left(h_{0} \frac{k}{\kappa^{2}} E\right)_{, x}+\frac{l}{h_{0}}\left(h_{0} \frac{l}{\kappa^{2}} E\right)_{, y}
\end{aligned}
$$

Here $\kappa^{2}=|\vec{k}|^{2}$. These estimates can be useful for scaling the magnitude of the vorticity deposition as a function of energy density. In our numerical experiments, the mean field vorticity is directly available, but equations 15 and 16 can be used to think about some simple cases, and to check the numerics. Moreover, these estimates allow extension of the numerical model results to the cases of stronger wave forcing than is allowed by the numerical model.

In the case of a normally incident wave $(l=0)$ with an envelope $\left(\frac{\partial}{\partial y} \neq 0\right)$ over longshoreindependent terrain (see Figure 5), the vorticity source term is

$$
(\nabla \times \bar{u})_{, t}=-\frac{k}{h_{0}}\left(\frac{h_{0}}{k} E\right)_{, x y}
$$

Aside from factors which arise from topographic variation, the right hand side is due to the $y$-variation of energy density divergence $E_{, x}$. Hence $\nabla \times \bar{u} \sim T \frac{E}{X Y} \sim T \frac{c_{00}^{2}}{h_{0}} \frac{\overline{h^{\prime 2}}}{X Y}$. Here $X$ and $Y$ are length scales for change of $E$, and $T$ is the total forcing time. The presence of the cross-derivative and the sign of the result must be diagnosed from the situation. Since we expect the amount of energy dissipation to be proportional in some way to the amplitude, the vorticity signal is as in Figure 5.

In the case of an obliquely incident wave $(l \neq 0)$ with no envelope $\left(\frac{\partial}{\partial y}=0\right)$ over longshore-independent terrain, the vorticity source term is

$$
(\nabla \times \bar{u})_{, t}=-\frac{l}{h_{0}}\left(\frac{h_{0} k}{\kappa^{2}} E\right)_{, x x}
$$

The primary signal is in the rate of change along the x-axis of $E_{, x}$, but the scaling now includes a geometric factor: $\nabla \times \bar{u} \sim T \frac{k l}{\kappa^{2}} \frac{E}{X^{2}} \sim T \frac{l k}{\kappa^{2}} \frac{c_{00}^{2}}{h_{0}} \frac{\overline{h^{2}}}{X^{2}}$. This idealized situation is depicted in Figure 6.

We conclude this section by noting a relevant result that D.H. Peregrine has obtained. He used the circulation integral to give the total change in vorticity that a parcel of infinitesimal extent experiences upon passing through a bore [1]:

$$
\delta(\nabla \times \vec{u})=\left[\frac{2 h_{2}}{g h_{1}\left(h_{1}+h_{2}\right)}\right]^{\frac{1}{2}} \frac{d E_{D}}{d y}
$$

Here, $E_{D}$ is the energy dissipation rate at the bore, $h_{2}$ and $h_{1}$ are fluid depths to the right and left of the bore, respectively. We take $h_{2}>h_{1}$ and the parcel crossing from left to right. There is an additional change $\Delta$ in vorticity due to the stretching of the parcel as it crosses the bore. $\Delta$ is proprtional to $\left(\frac{h_{2}}{h_{1}}-1\right)(\nabla \times \vec{u})$. Notice the dependence on the along-bore change in energy dissipation. 


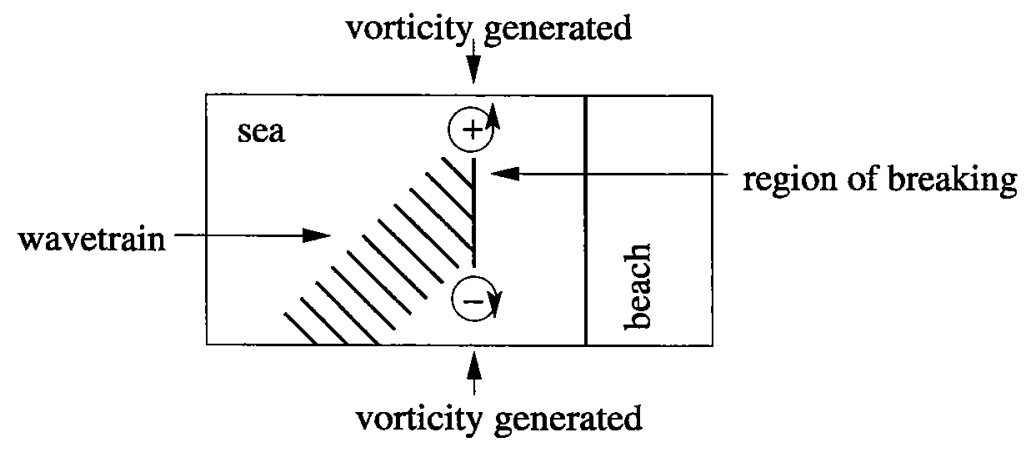

Figure 5: Enveloped incident waves. The component of the vorticity due to breaking which is associated with the envelope appears as the signed circles at the margins of the envelope.

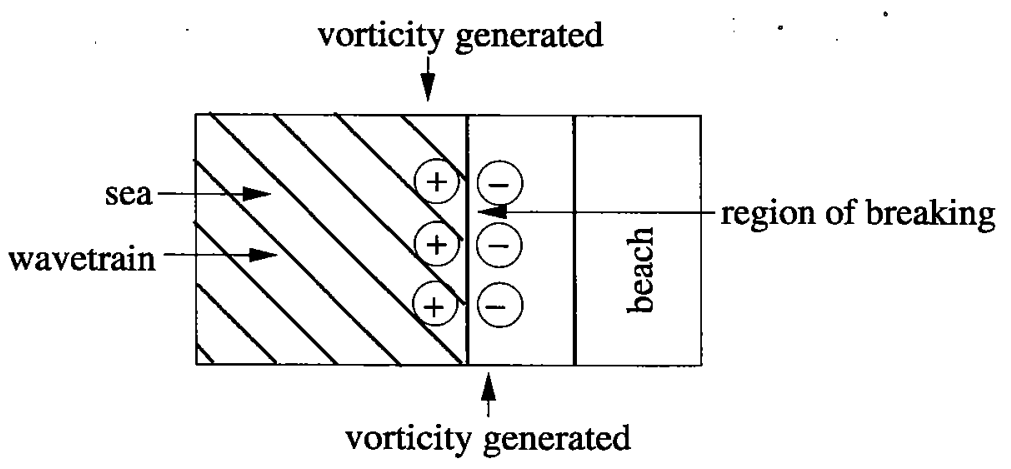

Figure 6: Unenveloped incident waves. The vorticity associated with the restriction of the breaking region to the region over the bar appears as the signed circles to the left and right of the bar. 


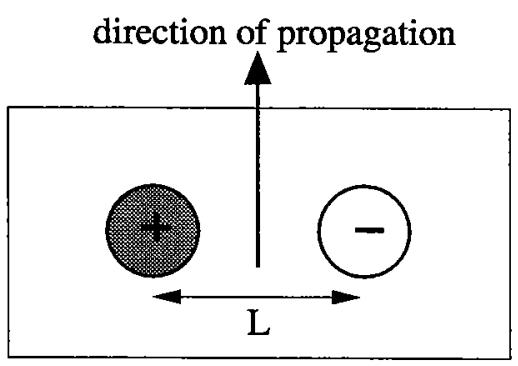

Figure 7: A vortex pair. Plan Form.

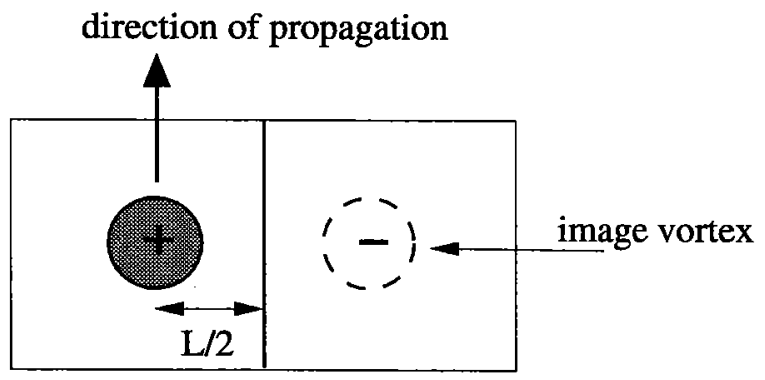

Figure 8: Vortex near a wall. Plan Form.

\section{Vortex Dynamics}

Consideration of the dynamics of a few typical vortical structures helps explain the motions associated with wave-induced mean-flow vorticity.

The motion of a vortex pair of opposite sign is perpendicular to the joining axis, see figure 7. The motion is in the direction indicated, with a velocity $V$ which scales with $S / L$, where $S$ is the vortex strength (i.e. circulation), and $L$ is the separation. A typical timescale is $T \sim L / V=L^{2} / S$.

The motion of a single vortex adjacent to a wall is found from the method of images. It is just that of a vortex pair whose joining axis is perpendicular to the wall, and which has midpoint on the wall. (See Figure 8.)

The motion of a vortex on a slope is also found by a method of images: the vortex is completed to a vortex ring outside the domain. (Figure 9.) The vortex moves under the velocity field of this ring, perpendicular to the gradient of the slope. This result is not useful for a line vortex ring since such a ring moves with infinite velocity $([11])$; instead, one should consider a distributed vortex patch. Stable vortex ring cross-sections are not known analytically, but one can estimate the speed of travel by cutting off the logarithmic divergence of the longshore velocity at the edge of the vortex core.

The distributions of vorticity in figure 5 of the previous section is such that the vortex pair should move up-slope over the bar, and towards the beach. However, the vortices should separate as they move up-slope, and come together as they move down-slope, in correspondence with the motion of figure 9.

The vorticity distribution in figure 6 is such that there is no apparent self-advection of vorticity, but a jet is driven between left and right vortex line. This situation would be 


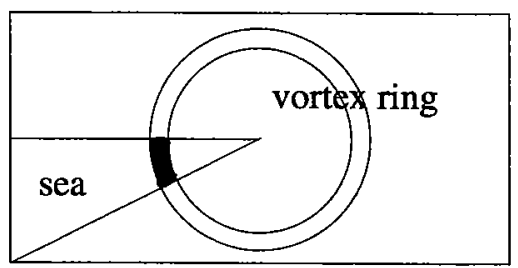

Figure 9: A vortex ring. The cross-section is the plane perpendicular to the axis of the beach. Motion of the vortex core is into or out of the page according to the sign of the vorticity.

unstable in the absence of mixing and bottom friction.

\section{Numerical Results}

We present two sets of results from the numerical experiments. The first is for the case of an obliquely incident wavetrain incident on a barred beach, and corresponds to the idealization of Figure 5. The second is the $y$-homogeneous case of Figure 6, again on a barred beach.

\subsection{Numerical Model}

The model is a shock-resolving finite-volume shallow-water solver built for this project. It uses a second-order time-stepping method, with the inter-cell fluxes computed by a Roeapproximate scheme. The topographic momentum source terms are handled with a splitting scheme. The CFL number is mantained at 0.95 throughout, with wavespeeds updated after each time-step. The waves are generated at the left side of the computational domain by an irrotational momentum forcing, derived from a potential. The forcing site introduces negligible vorticity into the simulations. The forcing generates a left- and a right-propagating wavetrain. The rightwards-moving wavetrain survives to break at the bar, but the other is removed by a damping layer at the left edge of the computational domain. The right side of the computational domain is a reflecting boundary condition. All runs were performed with shallow water $\left(<0.1 h_{\max }\right)$ near this right edge, so that waves of significant amplitude are removed by breaking at this location. The model is periodic in the longshore direction. The model has two major limitations. Only small-amplitude waves $\left(h^{\prime} / h_{0} \sim 0.01\right)$ can be used, since waves of a larger order of magnitude shock fully after a single wavelength. This is in contrast with ocean waves, which experience no difficulty in propagating through deep water, only to break with $O(1)$ nonlinearity in the surf zone. Second, the requirement that the model resolve gravity waves led to small time-steps, so that integration of the vortex dynamics became computationally expensive. Pertinent information about the model runs is summarized in Table 1.

\subsection{Enveloped oblique wavetrain}

In this model run, a wavetrain is incident on the bar at an angle of 74 degrees from the normal. Figures 6.2 shows a contour plot of the free surface height. The PV field and the 


\begin{tabular}{lllllll}
\hline Type & $k$ & $l$ & $k_{a b s}$ & Frequency & Envelope (half-width) & Amplitude \\
\hline Enveloped & $\pi$ & $0.3 \pi$ & $1.044 \pi$ & $1.044 \pi$ & 6 & 0.01 \\
Homogeneous & $\pi$ & $0.5276 \pi$ & $1.131 \pi$ & $1.131 \pi$ & None & 0.01 \\
\hline
\end{tabular}

Table 1: Computations with and without envelope.

longshore current appear also in figure 6.2. Notice the appearance of the dipolar vorticity signal and its subsequent self-advection into the trough region. The longshore current is itself a consequence of another vortical dipole structure, though this is visible only in the tilting of the much stronger cross-shore-axis dipole. The average longshore velocity maximum clearly moves into the trough, though the average velocities are pathetic compared with local mean flow velocities (Figure 6.2). Much larger incident wave amplitudes would be needed to generate the longshore currents seen in the real surf zone, though whether the overall structure of the flow would be maintained in this case is unclear. In any case, this is the mechanism proposed for the presence of longshore currents in the trough. Ultimately the vortex pair curls up at heads which separate on the shore slope of the beach. They begin to feel the periodicity of the domain (Figure 6.2). At this point, the applicability of the model is ended and the run is termianated.

\subsection{Homogeneous oblique wavetrain}

This case has a homogeneous wave structure incident at 60 degrees from the normal. This case is intended as a check against the experimental work of Reiners and Battjes. [5]. The wavefield develops significant interaction with the mean flow after some initial development (Figure 13). Likewise, an intially extremely clean signal of PV its longshore jet are seen to become unstable in time. (Figure 14) The experimental work in [5] shows little evidence of this instability, but that work included bottom friction and momentum diffusion as an experimental matter of course, but such things as are not present in the numerics. The numerically produced longshore currents remain of lower amplitude (by a factor of six) than those of Reiners and Battjes, but half of this disagreement disappears when the numerical results are rescaled by $\sqrt{g}$ to include gravity in the shallow-water wave speed. If the jet had been stabilized by dissipation and forced at a higher wave amplitude, a result close to that of the experimental work might have been obtained. However, the forced-dissipative regime may be rather different from the numerical regime.

\section{Conclusion}

The main limitations of the numerical model are its inability to deal with large amplitude waves, and its failure to include dissiption and an eventual forced-dissipative equilibrium. The latter effect is easily incoporated into the model and should be included in any future work. The problem of amplitude is probably best approached by considering scaling arguments with respect to the wave amplitude $h^{\prime}$. Questions of three-dimensional structure may be of interest, but lie beyond the scope of this study. The same is true of issues regarding the space- and time-distributions of incident waves. Such effects could be incorporated in 

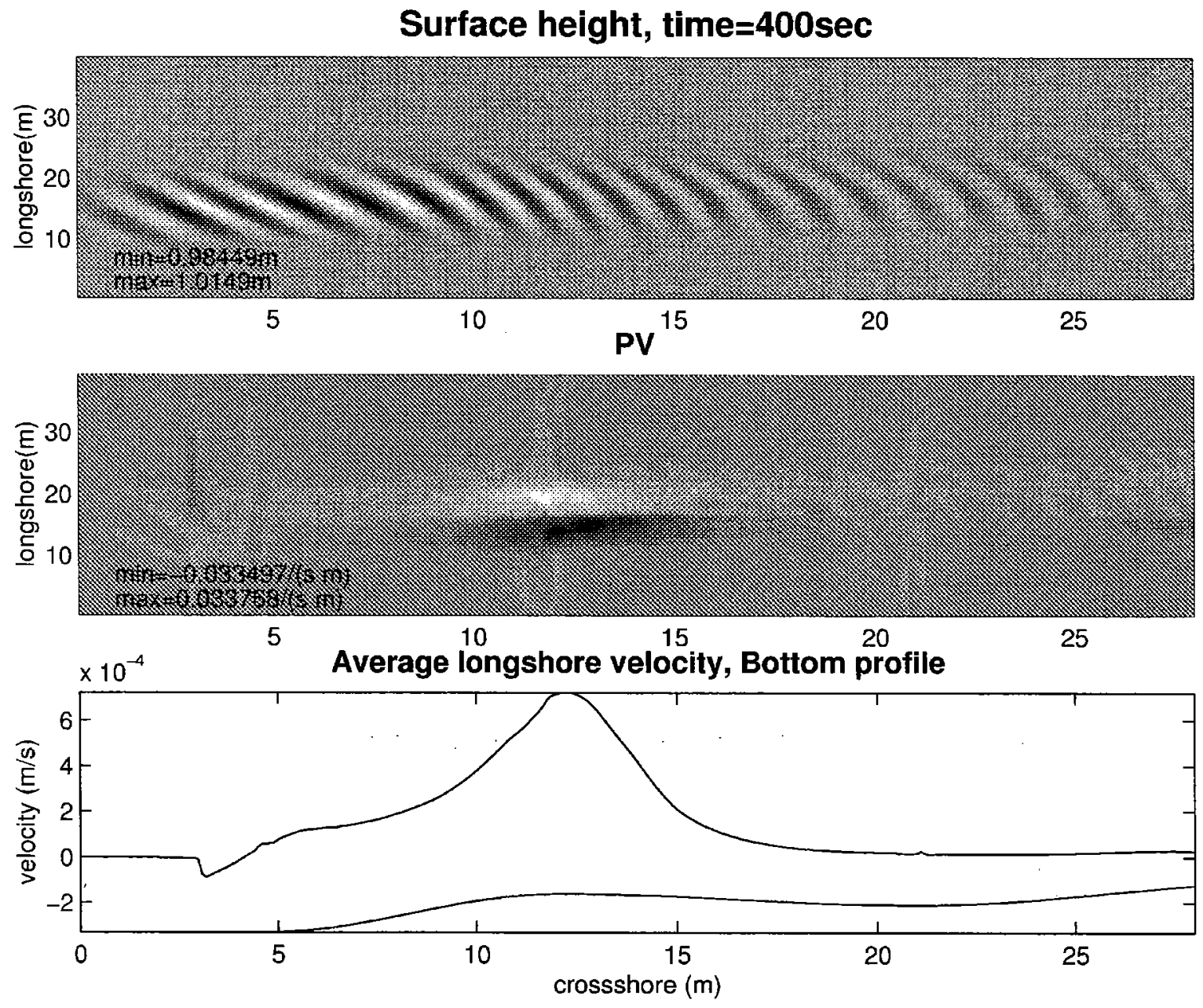

Figure 10: The enveloped oblique wavetrain. (See section 6.2.) A view of the height, PV, and average velocity in the early stage of development... 
Surface height, time $=2000 \mathrm{sec}$
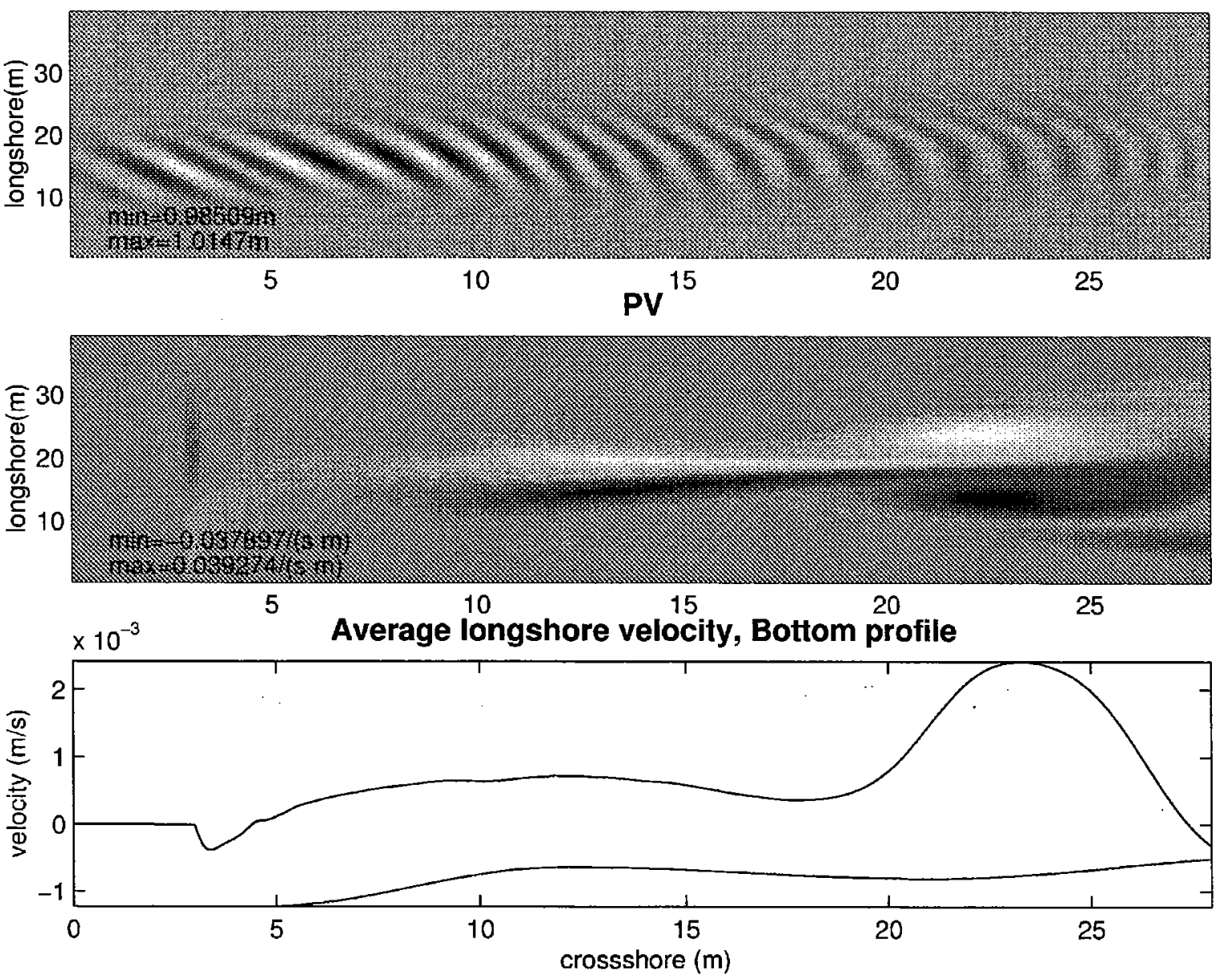

Figure 11: ...later... 

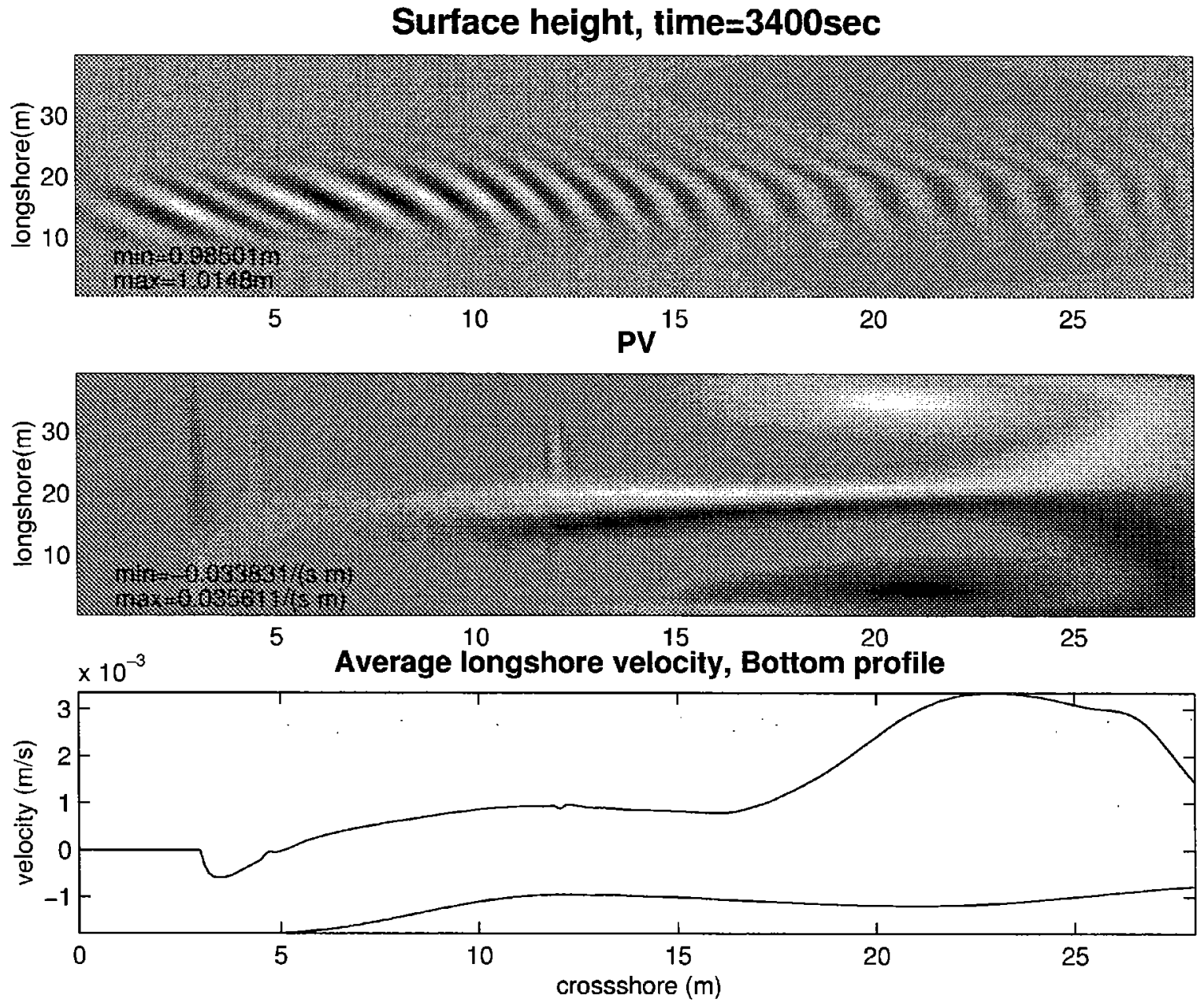

Figure 12: ...right before model termination. 

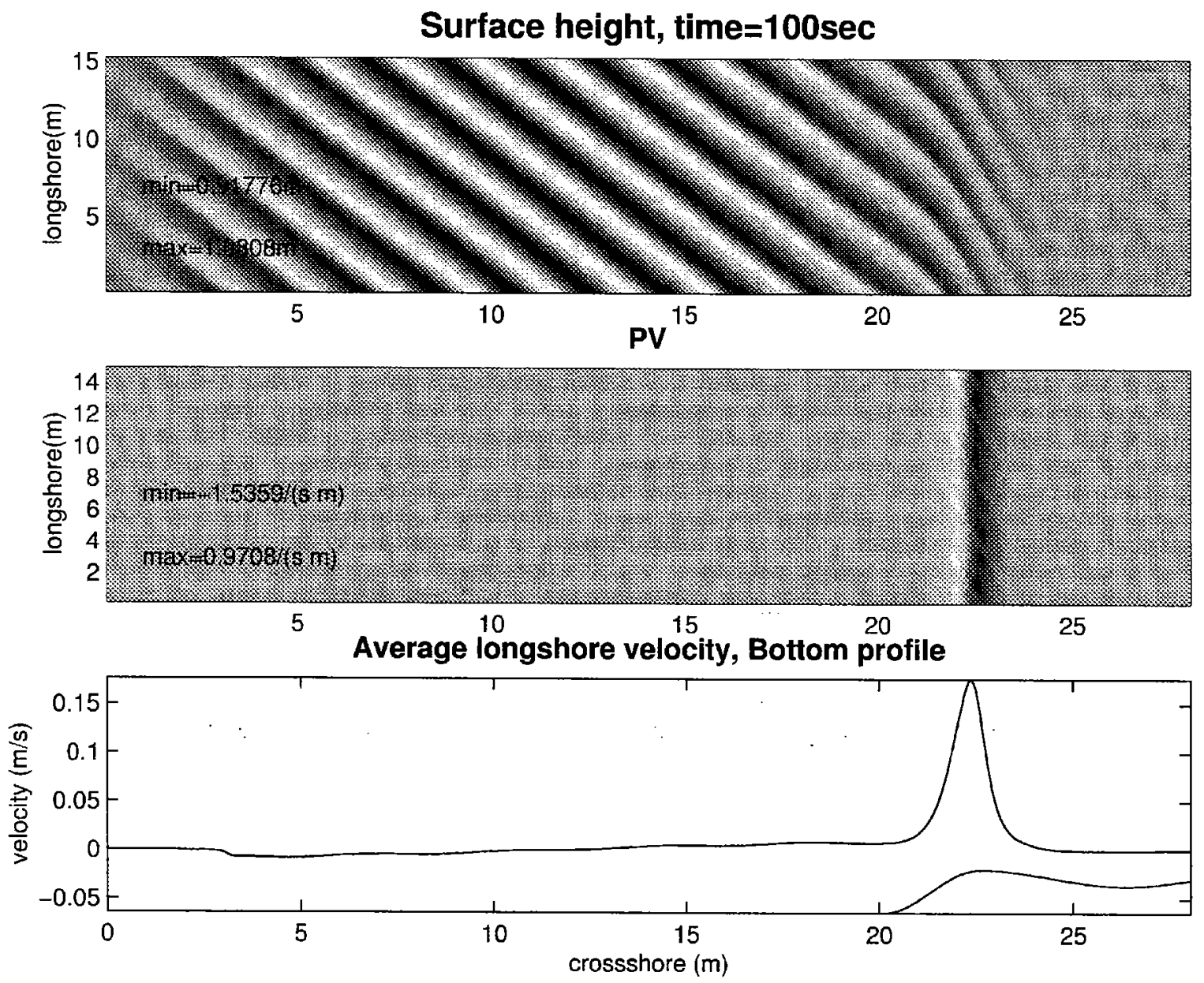

Figure 13: The homogeneous oblique wavetrain. (See section 6.3.) A view of the height, $\mathrm{PV}$, and average velocity in the early stage of development... 

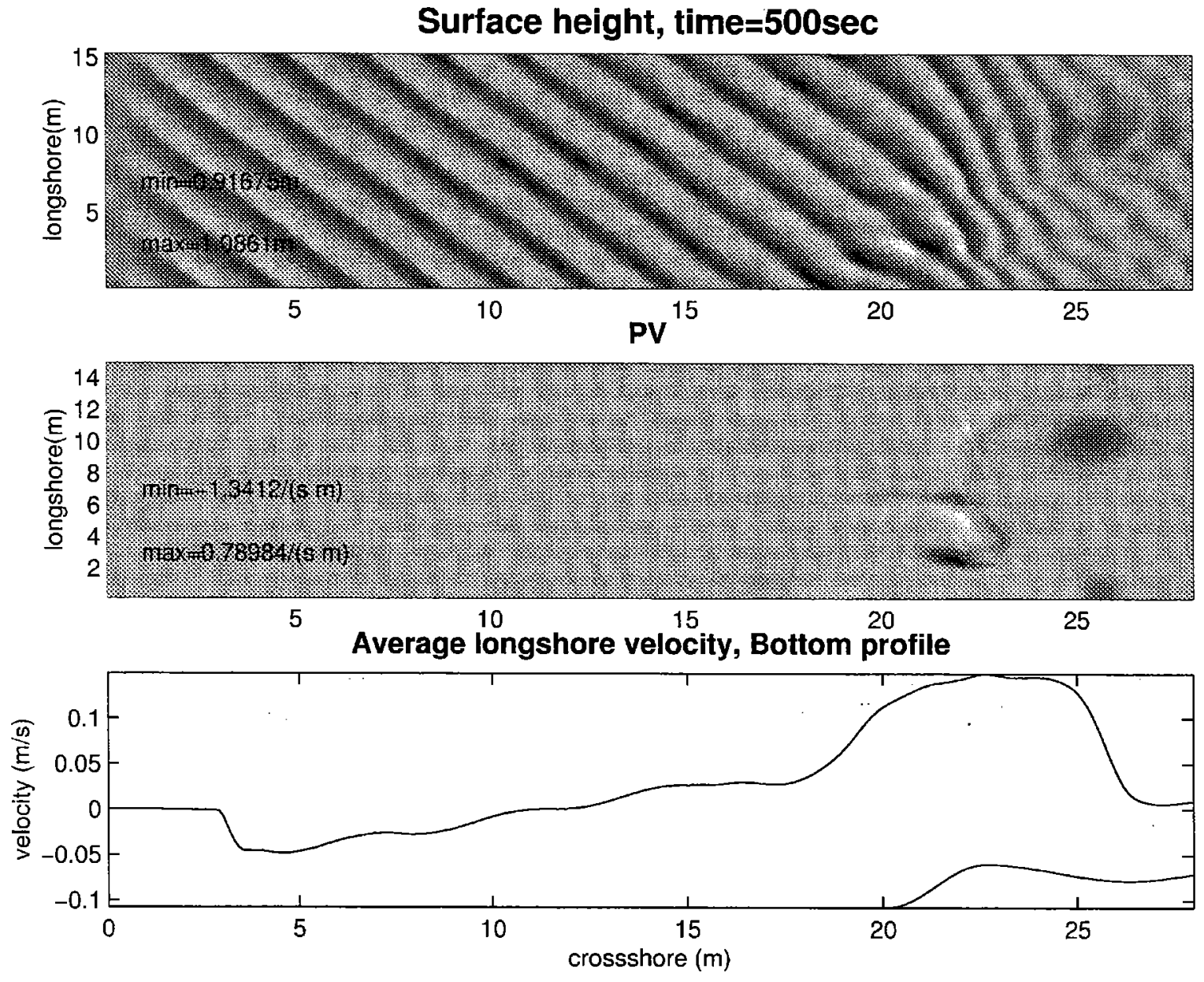

Figure 14: ...after the jet has grown unstable. 
the numerical model, but are computationally much more demanding. Typical integration times for this model were already on the order of 10-20 hours.) An interesting topic for future research would be an attempt, probably based at first on a WKB approximation and a breaking parameterization, to identify interesting coupled wave-mean interactions. One could also consider the motion of the sand bars as a further mediation between wave and mean.

\section{References}

[1] D. H. Peregrine, "Surf zone currents," Theoretical and Computational Fluid Dynamics 10, 295 (1998).

[2] D. H. Peregrine, "Large scale vorticity generation by breakers in shallow and deep water," Eur. J. Mech. B/Fluids 18, 403 (1999).

[3] F. Feddersen (unpublished).

[4] J. C. Church and E. B. Thornton, "Effects of breaking wave induced turbulence within a longshore current model," Coastal Engineering 20, 1 (1993).

[5] A. H. J. M. Reniers and J. A. Battjes, "A laboratory study of longshore currents over barred and non-barred beaches," Coastal Engineering 30, 1 (1997).

[6] G. B. Whitham, Linear and Nonlinear Waves (John Wiley and Sons, New York, 1974).

[7] O. Bühler, "On the vorticity transport due to dissipating or breaking waves in shallowwater flow," J. Fluid Mech. 407, 235 (2000).

[8] D. G. Andrews and M. E. McIntyre, "On wave-action and its relatives," J. Fluid Mech. 89, 647 (1978).

[9] D. G. Andrews and M. E. McIntyre, "An exact theory of nonlinear waves on a lagrangian-mean flow," J. Fluid Mech. 89, 609 (1978).

[10] O. Buhler and M. E. McIntyre, "On non-dissipative wave-mean interactions in the atmosphere or oceans," J. Fluid Mech. 354, 301 (1998).

[11] G. K. Batchelor, An Introduction to Fluid Dynamics (Cambridge University Press, Cambridge, 1967). 


\title{
A Truncated Model for Finite Amplitude Baroclinic Waves in a Channel
}

\author{
Zhiming Kuang
}

\section{Introduction}

To date, studies of finite amplitude baroclinic waves have been mostly numerical. The numerical models, ranging from general circulation models (GCMs) to two-layer quasigeostrophic (QG) models have been able to simulate certain observed features of the midlatitude synoptic system quite well. For instance, the asymmetric life cycle of global normal modes observed by Randel and Stanford [1] has been well simulated by Simmons and Hoskins [2] with a primitive equation model and by Feldstein and Held [3] with a 2-layer QG model. Coherent wave packets that are observed in both the Northern Hemisphere (NH) and the Southern Hemisphere (SH) have also been simulated in a hierarchy of models $[4,5]$. Analytical theories of finite amplitude baroclinic waves, on the other hand, have not been developed except for those under weakly nonlinear conditions. The requirement of small super-criticality severely limits the application of such a theory on the real atmosphere, which clearly exhibits finite super-criticality.

This work attempts to construct a truncated 2-layer QG model that is capable of capturing the essential features of finite amplitude baroclinic waves. The existence of such a model is suggested by the remarkably simple meridional structures of the nonlinear baroclinic waves as simulated by untruncated 2-layer QG models [5]. Successful truncation may provide insight into the underlying processes, and might lead a step towards an analytical theory for the finite amplitude barolinic waves.

\section{Model}

The 2-layer QG equations on a $\beta$-plane may be written as follows:

$$
\begin{gathered}
\frac{\partial q_{1}}{\partial t}+J\left(\psi_{1}, q_{1}\right)=r\left(\frac{\psi_{1}-\psi_{2}}{2}-\tau_{e}\right)-\nu \nabla^{6} \psi_{1} \\
\frac{\partial q_{2}}{\partial t}+J\left(\psi_{2}, q_{2}\right)=-r\left(\frac{\psi_{1}-\psi_{2}}{2}-\tau_{e}\right)-\kappa_{M} \nabla^{2} \psi_{2}-\nu \nabla^{6} \psi_{2}
\end{gathered}
$$

where

$$
q_{j}=\beta y+\nabla^{2} \psi_{j}+(-1)^{j}\left(\frac{\psi_{1}-\psi_{2}}{2}\right), j=1,2
$$


Here, $\mathrm{j}=1$ and 2 refer to the upper and lower layers. $\kappa_{M}$ is the Ekman friction, $\mathrm{r}$ is the relaxation rate towards the equilibrium state $\tau_{e}, \nu$ is the numerical diffusion that parameterizes cascade to unresolved scales. The equations have been nondimensionalized. The radius of deformation $\lambda$ is chosen as the horizontal length scale, where

$$
\lambda^{2}=g\left(\rho_{2}-\rho_{1}\right) H /\left(2 \rho_{2} f_{0}^{2}\right)
$$

and $\mathrm{H}$ is the depth of either layer. Time is scaled by $\lambda / U_{0}$, where $U_{0}$ is the horizontal velocity scale. For more detailed discussion about this model, the reader is referred to Lee and Held (1993) [5].

I choose the boundary conditions that correspond to a channel with rigid walls [6]:

$$
\frac{\partial \psi_{j}}{\partial x}=0 \& \frac{\overline{\partial \psi_{j}}}{\partial y}=0 \quad y=0, L_{y}
$$

$\mathrm{x}$ is the direction along the channel (zonal, longitude) and $\mathrm{y}$ is the direction across the channel (meridional, latitude). This choice is supported by the fact that wave packets are more coherent when the storm track is more meridionally confined. The general solution that satisfies the boundary conditions can be written as follows:

$$
\begin{aligned}
& \psi_{j}(x, y, t)= \sum_{m=1}^{\infty} A_{j, m}^{\prime}(x, t) \sin \frac{m y}{N}+\sum_{n=1}^{\infty} C_{j, n}(t) \cos \frac{n y}{N} \\
&+\sum_{m=1}^{\infty} \overline{A_{j, m}}(t)\left(\sin \frac{m y}{N}-\frac{m y}{N}+[(m \bmod 2)-1] \frac{m}{N} \frac{y^{2}}{N \pi}\right)
\end{aligned}
$$

where $A_{j, m}^{\prime}(x, t)=A_{j, m}(x, t)-\overline{A_{j, m}}$. The overbar denotes the average over x. I have chosen $L_{y}$ to be $N \pi \lambda$.

\section{3 "Rules" for Truncation}

To construct a truncated model, one needs to first select a desirable subset of base functions, then express the unknowns in terms of these base functions and substitute them into the equations. One then projects the equations onto the selected base functions to obtain a new set of equations to solve for the coefficients of the base functions.

To be "desirable", first of all, the truncation needs to be simple, as the whole goal of truncation is simplification, or to capture essential features of the system with less degrees of freedom. It is also desirable for the truncated models to retain the conservation laws of, for example, energy, potential enstrophy, and momentum. Even though they are not required, as the truncated models are, by nature, approximations, conservation laws, if retained, prove to be very useful for model validation and facilitate the discussion of, for instance, the energy conversion.

It can be shown that if energy and potential enstrophy, or in general, any quadratic quantities, are conserved for a base function set, they are also conserved by a subset that consists of orthogonal base functions. One complete set of base functions, suggested by the general solution (6), includes:

$$
()^{\prime} \sin \frac{m y}{N}, m=1, \infty
$$




$$
\begin{gathered}
\overline{(}\left(\sin \frac{m y}{N}-\frac{m y}{N}+[(m \bmod 2)-1] \frac{m}{N} \frac{y^{2}}{N \pi}\right), m=1, \infty \\
\overline{()} \cos \frac{n y}{N}, n=1, \infty
\end{gathered}
$$

Modes in set 7 may be called eddy base functions and have zero $x$ averages. Set 8 and 9 consists of base functions that represent the zonal mean components. As $\int_{0}^{N \pi} \sin \frac{m y}{N} \sin \frac{l y}{N} d y=$ 0 , when $m \neq l$, and $\int()^{\prime} \overline{()} d x=0$ by definition, every base function in (7) is orthogonal to all the other base functions, including those in (8) and (9). Base functions in (9) are orthogonal to other base functions within set 9 yet not orthogonal to base functions in set 8. Base functions in set (8) are not orthogonal to each other. Therefore, any combination of set 7 base functions together with any combination of set 9 base functions or one set 8 base function would be an orthogonal set. Here, I choose set 9 to represent the zonal mean components as it appears to be simpler than set 8 .

When there is no friction, the untruncated model should conserve total momentum

$$
\frac{d}{d t} \int_{0}^{N \pi} \frac{\partial \overline{\psi_{M}}}{\partial y} d y=0
$$

where subscript $M$ denotes the barotropic component. Adding equation (1) and equation (2) gives

$$
\frac{\partial q_{M}}{\partial t}+J\left(\psi_{M}, q_{M}\right)+J\left(\psi_{T, q_{T}}\right)=0
$$

Subscript $\mathbf{T}$ denotes the baroclinic component. It is clear that changes in the zonal mean barotropic components can only come from wave-wave interactions represented by the Jacobian of eddies. Since the eddies have base functions of the form ()$^{\prime} \sin \frac{m y}{N}$, if the indexes (m's) of any pairs of eddy base functions are separated by odd numbers and set 9 is selected as the base functions for the zonal components, it can be shown that the Jacobians in Eq. 11 do not affect the channel integrated barotropic zonal velocity, i.e. they conserve the total momentum (Appendix). The same is not true when set 8 is used or when the indexes of the eddy base functions are separated by even numbers. It directly follows that the selected eddy base function set should have no more than 2 base functions, for with more than 2 base functions, there must be at least one pair whose indexes are separated by an even number.

\section{Truncation I}

Following the "rules" described in the previous section,

$$
\left\{()^{\prime} \sin \frac{y}{N},()^{\prime} \sin \frac{2 y}{N}, \overline{()} \cos \frac{y}{N}, \overline{()} \cos \frac{2 y}{N}\right\}
$$

appears to be an appropriate set. The unknown variables are then expressed in terms of these base functions.

$$
\psi_{j}(x, y, t)=A_{j, 1}^{\prime}(x, t) \sin \frac{y}{N}+A_{j, 2}^{\prime}(x, t) \sin \frac{2 y}{N}+\overline{C_{j, 1}}(t) \cos \frac{y}{N}+\overline{C_{j, 2}}(t) \cos \frac{2 y}{N}
$$




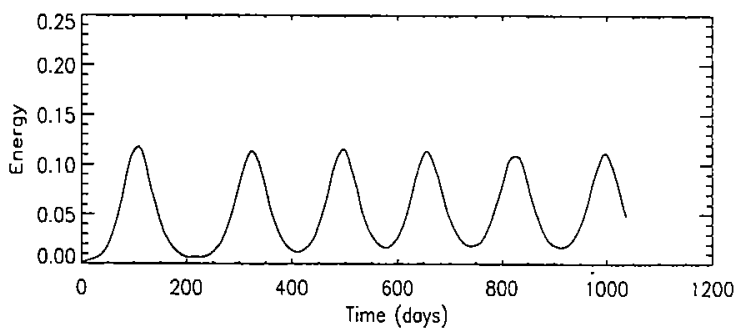

(a)

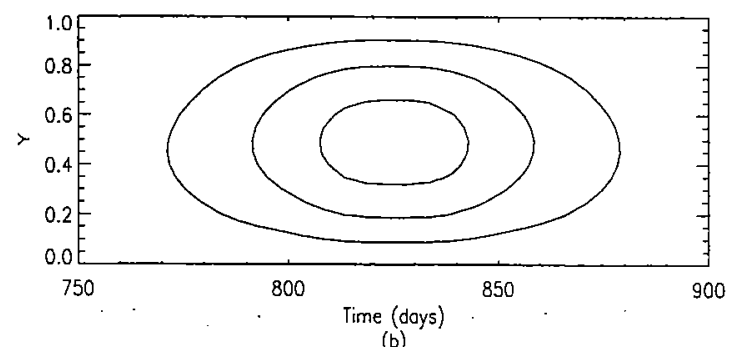

(b)

Figure 1: Time evolution of a normal mode with a weakly supercritical background zonal flow. (a) shows the total wave energy (solid), the baroclinic mean flow energy (dotted) and the barotropic mean flow energy (dashed). The absolute values of baroclinic mean flow energy have been shifted in the plot. (b) shows the latitude-time contour of zonally averaged upper layer wave streamfunction squared (i.e. variance) over one lifecycle of the normal mode. The unit in the y direction is $2 \pi \lambda$. 
The prime over $A_{j, i}$ s and the overbar over $C_{j, i}$ s remind us that $\mathrm{A}$ represents deviations from the zonal average and $\mathrm{C}$ represents the zonal means $(\mathrm{i}=1,2)$. By Equation $3, q_{j}$, excluding the $\beta y$ term, can be expressed in terms of the following components:

$$
\begin{gathered}
()^{\prime} \sin \frac{y}{N}: \quad \frac{\partial^{2} A_{j, 1}^{\prime}}{\partial x^{2}}-\frac{A_{j, 1}^{\prime}}{N^{2}}+(-1)^{j} \frac{A_{1,1}^{\prime}-A_{2,1}^{\prime}}{2} \\
()^{\prime} \sin \frac{2 y}{N}: \quad \frac{\partial^{2} A_{j, 2}^{\prime}}{\partial x^{2}}-\frac{4 A_{j, 2}^{\prime}}{N^{2}}+(-1)^{j} \frac{A_{1,2}^{\prime}-A_{2,2}^{\prime}}{2} \\
\overline{()} \cos \frac{y}{N}: \quad-\frac{\overline{C_{j, 1}}}{N^{2}}+(-1)^{j} \frac{\overline{C_{1,1}}-\overline{C_{2,1}}}{2} \\
\overline{()} \cos \frac{2 y}{N}: \quad-\frac{4 \overline{C_{j, 2}}}{N^{2}}+(-1)^{j} \frac{\overline{C_{1,2}}-\overline{C_{2,2}}}{2}
\end{gathered}
$$

After substituting $\psi_{j}$ and $q_{j}$ into equations 1 and $2, \mathrm{I}$ project the equations onto the selected base functions. Take () $\cos \frac{y}{N}$ as an example, the projection is done by first multiplying the equations by $\overline{()} \cos \frac{y}{N}$, and then integrating over $\mathrm{y}$ and averaging over $\mathrm{x}$. Doing the projection for each base function and for both layers gives us 8 predictive equations for the 8 unknowns (the $A_{j, i} \mathrm{~s}$ and $C_{j, i} \mathrm{~s}$ ). I then solve the new equations numerically using the spectral method. The nonlinear Jacobian terms are computed using the spectral transform method. As the spatial dimension of the problem is reduced from 2 to 1 (there is no $\mathrm{y}$ dependence in the equations now), the implementation is greatly simplified. I implement the model in a way that the selected base function set needs not to be the one specified in (12), but can be any combination of set 7 and set 9 base functions.

I will first study the nonlinear initial value problem for this truncated model, and then study the forced and dissipated system. The channel width is set to be $2 \pi$ in this study.

\subsection{Normal mode study}

Without forcing and dissipation, I initialize the system with a zonal mean profile that is supercritical and perturb it with a ()$^{\prime} \sin \frac{y}{N}$ zonal wave disturbance that is close to the most unstable mode. The super-criticality and the most unstable mode are obtained from a linear stability analysis that I did for zonal wave disturbances with y dependence of the form $\sin \frac{m y}{N}$ and zonal background flows of the form $\cos \frac{n y}{N}$ (both in terms of stream-function).

Normal mode evolution with a weakly supercritical zonal flow has been studied using the weakly nonlinear theory. Figure 1 shows the time evolution of a normal mode with a weakly supercritical background zonal flow calculated from the truncated model. The stream-function of the background flow takes the form of $\overline{()} \cos \frac{y}{N}$. As described by the weakly nonlinear theory and simulated by untruncated 2 layer QG models, there is little change in the barotropic zonal flow energy. The eddies grow baroclinically and also decay baroclinically, exhibiting a symmetric life cycle in terms of wave streamfunction squared.

In the real atmosphere, however, normal modes are observed to have asymmetric life cycles. This is not explained by the weakly nonlinear theory. For eddies to decay barotropically, some of the eddy energy has to be converted into barotropic mean flow energy through irreversible mixing processes like wave breaking. Figure 2 shows the time evolution of a normal mode in the untruncated model when the super-criticality is raised. Greater changes in 


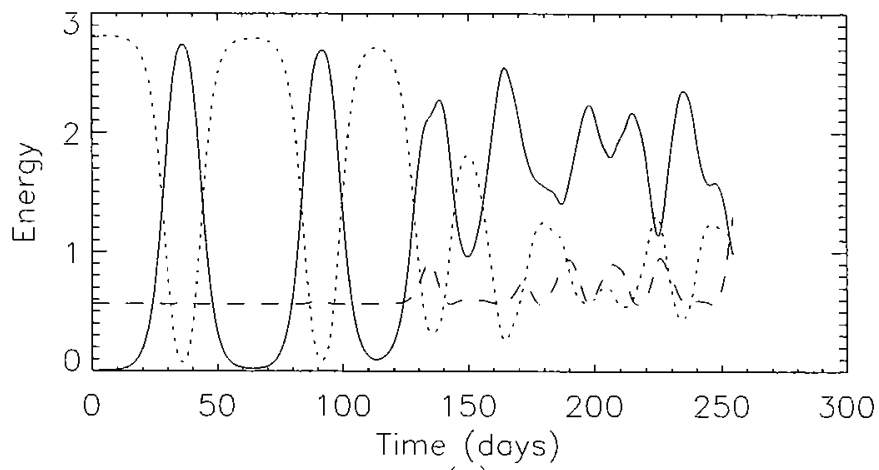

(a)

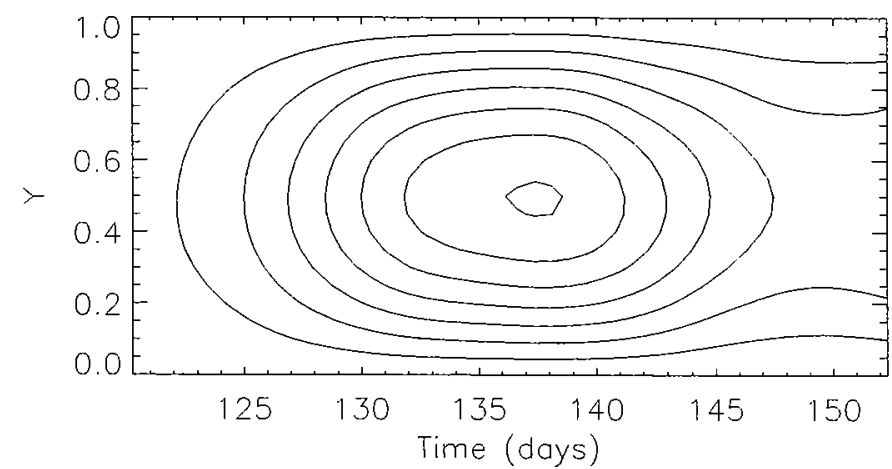

(b)

Figure 2: The same as Figure 1, except for the large super-criticality case.

the barotropic mean flow energy is observed. The life cycle becomes asymmetric as seen in the latitude-time contour of the zonal variance of the upper layer streamfunction. Changes in the barotropic mean flow energy are quite small however. So is the asymmetry of the life cycle. This should be expected since the truncated model has very limited degrees of freedom in the y direction, which limits its ability of mimicking fine scale processes like wave breaking. The model's ability of fully simulating the asymmetry in the eddy life cycles may be further limited by the $\cos \frac{y}{N}$ shape zonal mean stream-function that I have chosen. The resulted $\sin \frac{y}{N}$ shape zonal winds have rather weak meridional wind shear, which is thought to be critical for the barotropic decay of waves.

\subsection{Forced and dissipated systems}

I now examine the statistically steady state behaviors of finite amplitude wave when forcing and dissipation are present. The system is initialized with small noise like perturbations, with a Ekman friction $\kappa_{M}$ of 0.05 and a relaxation time of 50 model days (i.e. $\mathrm{r}=0.02$ ), and is relaxed towards the equilibrium profile $\tau_{e}$. The relative magnitude of $\kappa_{M} \mathrm{r}, \tau_{e}$ affects the shear strength of the statistically steady state. Here, I use the equilibrium profile $\tau_{e}$, which takes the form of $\cos \frac{y}{N}$, as a parameter to change the super-criticality of the statistically steady state. 

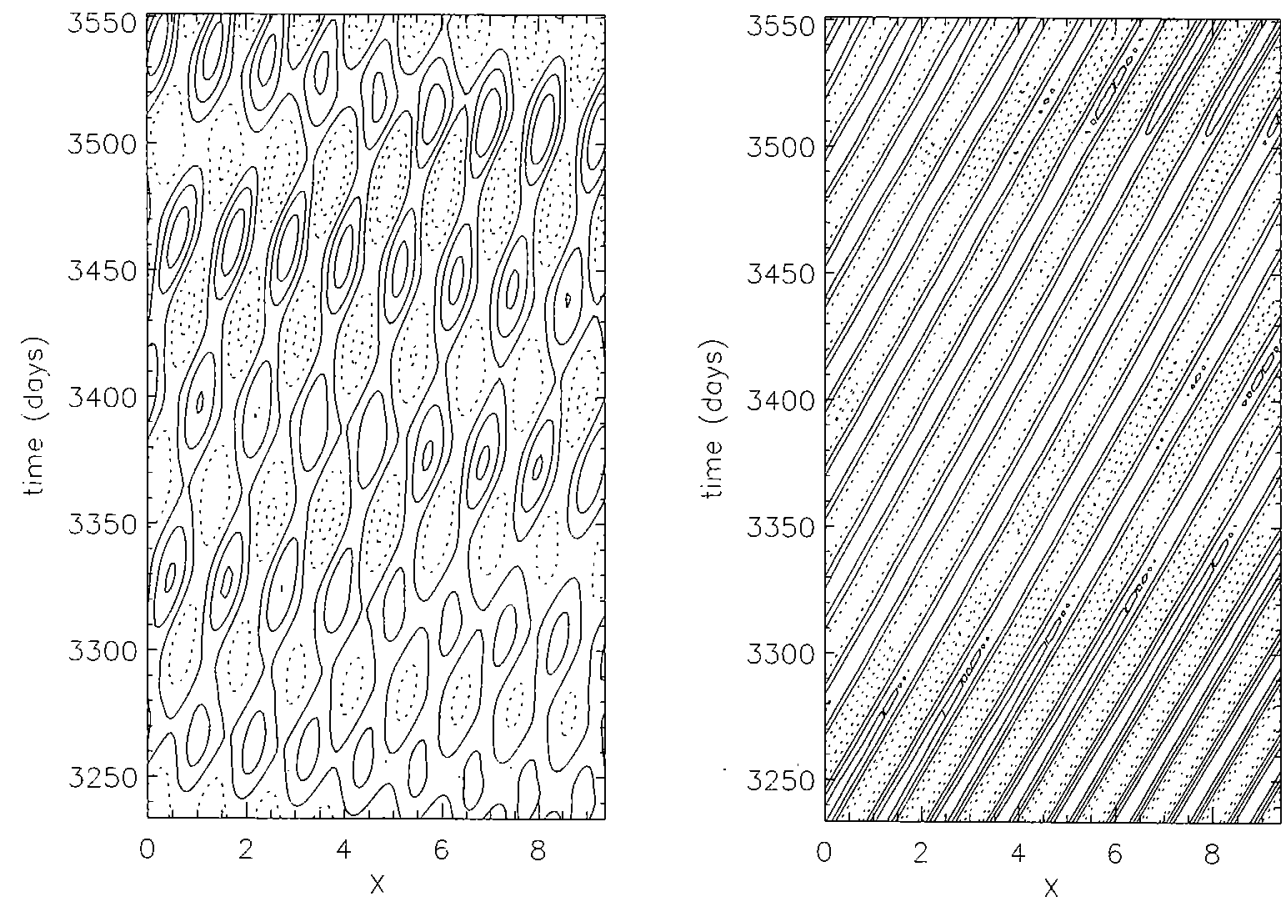

Figure 3: The longitude-time contour plots for the $\sin \frac{y}{N}$ (left) and $\sin \frac{2 y}{N}$ (right) modes of the upper layer stream-function for the weak super-criticality case. The unit of $x$ (longitude) is $2 \pi \lambda$. Negative contours are dotted. 

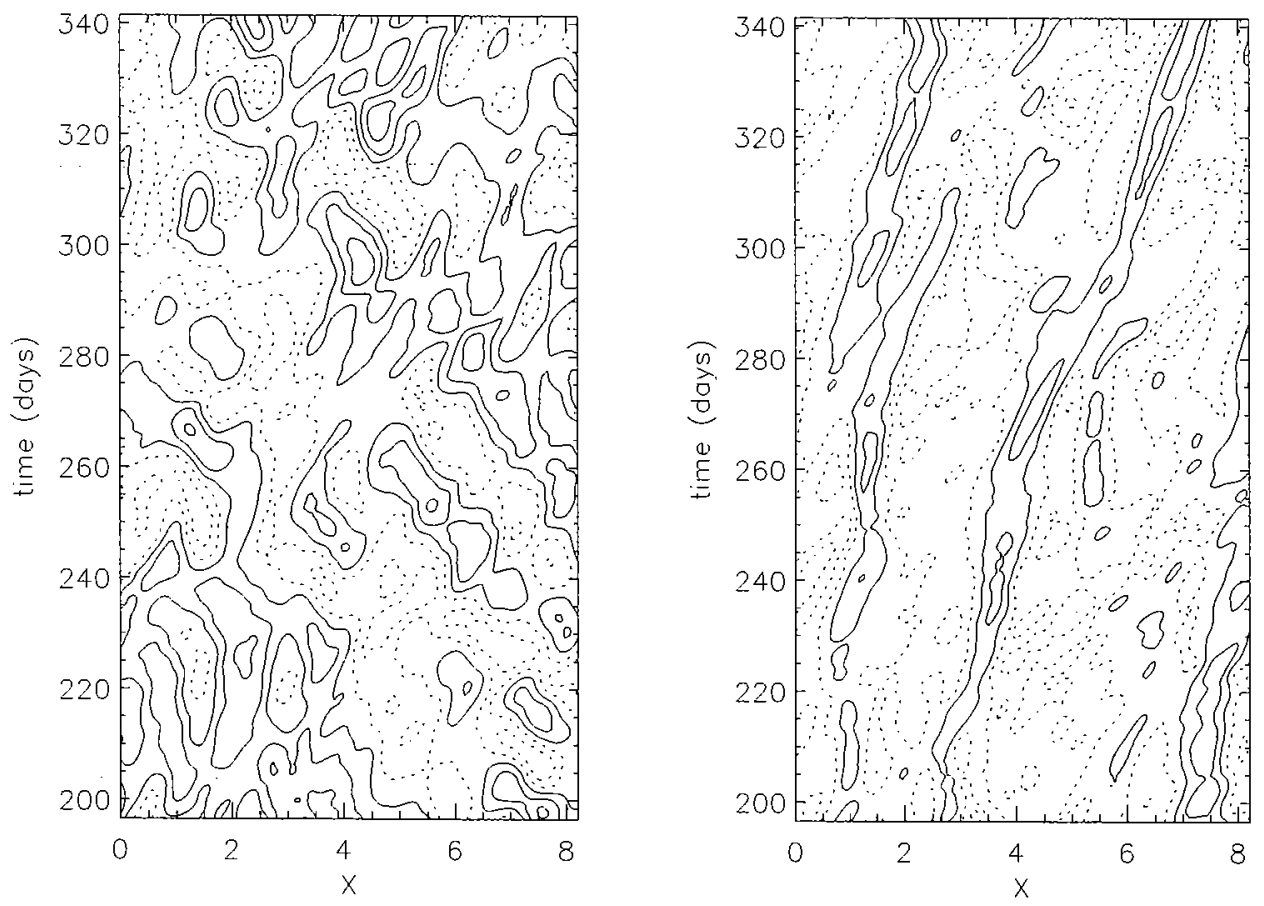

Figure 4: The same as figure 3, except for the strong super-criticality case.

The results from the truncated model are remarkably similar to those from an untruncated model study by Esler [7]. In Esler's study, the meridional structure is fully resolved by a 100 grid point finite differencing. An Empirical Orthogonal Function (EOF) analysis was then applied on the results to identify the dominant modes. Esler identified the first two modes as the antisymmetric mode and the symmetric mode, which have almost identical structures as the $\sin \frac{y}{N}$ and $\sin \frac{2 y}{N}$ modes used in this study. This result is not totally expected since although the $\sin \frac{y}{N}$ and $\sin \frac{2 y}{N}$ modes have the largest linear growth rates, whether they should still dominate when nonlinear effects become important is not totally clear. In figures 3 and 4, I present the longitude-time contour plots of the upper layer streamfunction for the cases of weak super-criticality (linear growth rate is about $0.02 /$ day) and strong super-criticality (linear growth rate is about $0.17 /$ day) respectively. For the weak super-criticality case, the wave train is modulated and the peaks appear to move at the same velocity as the phase speed. For the strong super-criticality case, the waves undergo quite chaotic evolution. Figure 3 may be compared to Esler's figure 15, and figure 4 may be compared to his figure 18. The remarkable similarity between the truncated model results and the untruncated model results strongly implies that the system has very low degrees of freedom in the meridional direction.

The model may be further reduced. Without the zonal mean component $\cos \frac{y}{N}$, one obtains results similar to those shown in figure 3 and 4 exceptthat the asymmetry of the eddy life cycles would not be simulated. Although not shown here, the potential vorticity (PV) fields in the upper and lower layers also resemble the results from Esler's study (his figure 
19) and show the expected behaviors. The PV field in the lower layer is homogenized and the PV gradient in the upper layer is strengthened with some indication of wave breaking.

\section{Other Truncations and Discussion}

Esler's model [7] does not show coherent wave packets. However, wave packets were found over a broad parameter regime by Lee and Held in their 2-layer QG model [5]. The two differences between their model and Esler's model are: (1) Lee and Held used a wider channel $(21 \lambda)$ than Esler did $(2 \pi \lambda)$; (2) Lee and Held used a Gaussian zonal wind shear while Esler used a uniform zonal wind shear. These two differences appear to be crucial for wave packet formation.

It turns out that the wave packets are readily formed if wave-wave interaction only affects the mean flow and does not change the eddies. This is sometimes called the quasilinear or wave-mean flow interaction model [3]. The simplest example of such a system has the following base function set:

$$
\left\{()^{\prime} \sin \frac{y}{N}, \overline{()} \cos \frac{y}{N}\right\}
$$

One can also choose, for example,

$$
\left\{()^{\prime} \sin \frac{y}{N},()^{\prime} \sin \frac{2 y}{N}, \overline{()} \cos \frac{y}{N}\right\}
$$

but ignore effects on the eddies by the wave-wave interaction, or choose, for example,

$$
\left.\left\{()^{\prime} \sin \frac{3 y}{N},()^{\prime} \sin \frac{4 y}{N}, \overline{(}\right) \cos \frac{3 y}{N}\right\},
$$

so that the wave-wave interactions do not project back onto the eddies themselves. Figure 5 shows the results for selection (13) as an example. The super-criticality here is much greater than that of the case in figure 4 (linear growth rate is about $1.9 /$ day), yet wave packets are found and exhibit great coherence.

For the base function sets specified in (13), (14) and (15), the behavior of wave packets closely resembles that described in Lee and Held (1993) [5]. For example, the number of wave packets is found to increase with increasing super-criticality and increasing channel length. The power spectrum of the eddies is also found to be very simple, as found by Lee and Held for the wave packets (e.g. their figure 11) [5], while truncation (I) gives very noisy spectrum when the super-criticality is high.

In all the quasi-linear models, one eddy mode interacts with other eddy modes and with itself to affect the evolution of the mean flow (nonlinear). The evolution of the eddies, however, is determined by the wave-mean interaction and is not directly affected by the wave-wave interaction. In these cases, the evolution of an eddy mode is in a sense independent of the other modes (of course, eventually they are coupled through the mean flow). The role of this "independence" in wave packet formation is unclear. Furthermore, from a weakly nonlinear analysis [7] and from the difference between Esler's model and Lee and Held model, it appears that the eddies need to be confined away from the walls for wave packets to form. Further understanding of these two points may provide us some hints on the mechanisms of wave packet formation. 


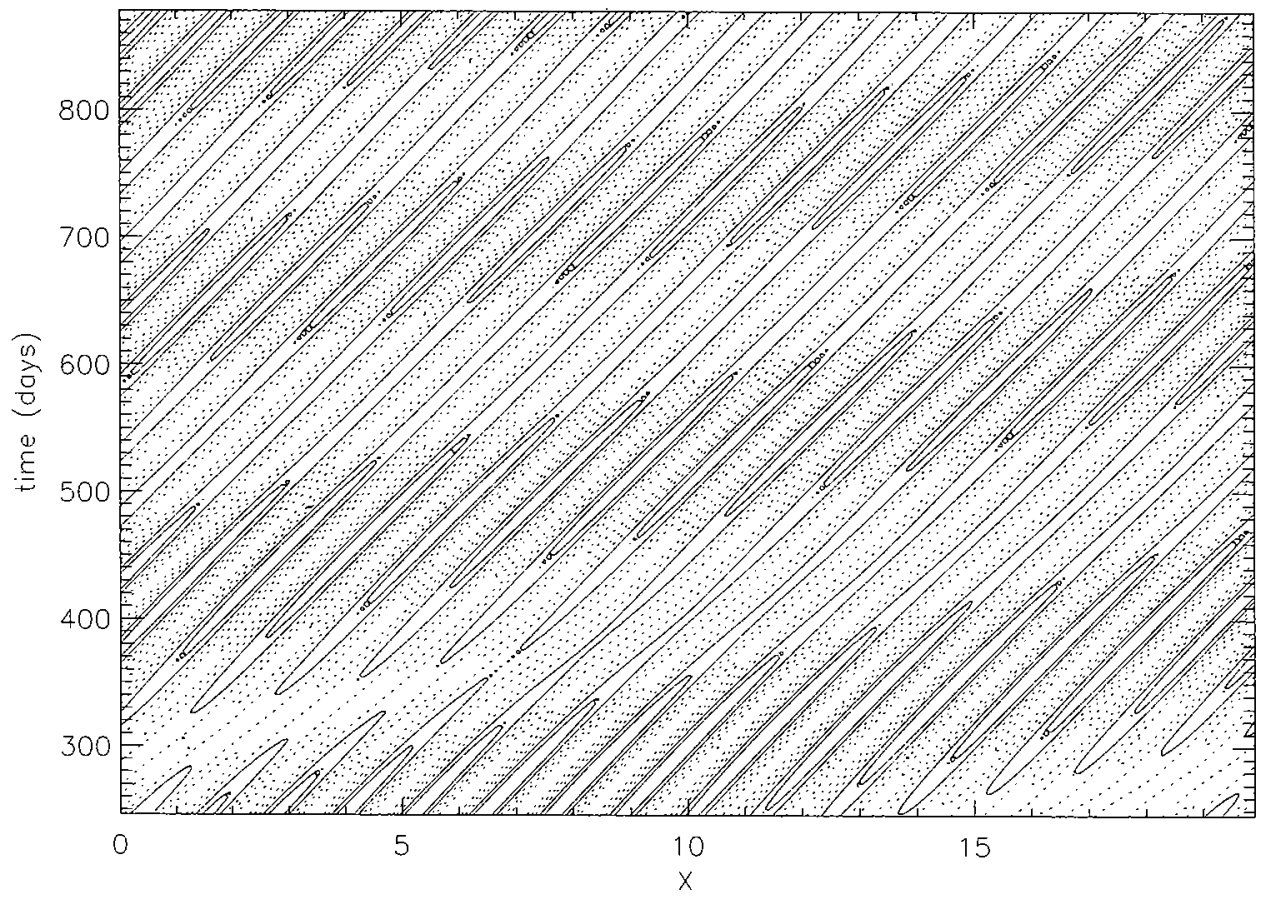

Figure 5: The longitude-time contour plot for the $\sin \frac{y}{N}$ mode of the upper layer streamfunction with base functions specified in (13). The unit of $\mathrm{x}$ is again $2 \pi \lambda$. 


\section{Concluding Remarks}

This work attempts to construct truncated 2-layer QG models for the study of finite amplitude baroclinic waves. I find that models severely truncated in the meridional direction are capable of capturing essential features of the fully resolved model. This is an indication of small degrees of freedom in the y direction. Truncation (I) is capable of simulating the asymmetric life cycle of the eddies, although only to a small extent due to the inefficiency of the truncated model in simulating fine scalewave breaking events. Coherent wave packets are found for truncated models that are quasi-linear, but not for models with wave-wave interactions in the eddy evolution equations. Coherent wave packets are also found for untruncated models with a wide channel and with a Gaussian zonal wind shear profile, but not for models with a narrow channel and with a uniform shear. Understanding these two differences may shed some lights on the mechanisms of wave packet formation. I also note that some important aspects of the problem have not been explored in this study, including, for instance, the effects of the channel width and the effects of different shapes of zonal wind profile.

\section{Acknowledgement}

I would like to thank Rick Salmon for suggesting this project to me and for "struggling" through the project with me. I would also like to thank Isaac Held for his great help on this project. He was very patient and helped me with many questions. I thank all the fellows and the staff members for this great summer, especially Chris Walker for the "fellow's car".

\section{Appendix}

Suppose that we take two eddy base functions ()$^{\prime} \sin \frac{m y}{N},()^{\prime} \sin \frac{l y}{N}$ so that

$$
\begin{gathered}
\psi_{M}^{\prime}=A_{M}^{\prime} \sin \frac{m y}{N}+B_{M}^{\prime} \sin \frac{l y}{N} \\
q_{M}^{\prime}=\left(\frac{\partial^{2} A_{M}^{\prime}}{\partial x^{2}}-\frac{m^{2} A_{M}^{\prime}}{N^{2}}\right) \sin \frac{m y}{N}+\left(\frac{\partial^{2} B_{M}^{\prime}}{\partial x^{2}}-\frac{l^{2} B_{M}^{\prime}}{N^{2}}\right) \sin \frac{l y}{N}
\end{gathered}
$$

Now we calculate $J\left(\psi_{M}^{\prime}, q_{M}^{\prime}\right)$. The Jacobian of the $\sin \frac{m y}{N}$ and the $\sin \frac{m y}{N}$ terms is

$$
\left\{\frac{\partial A_{M}^{\prime}}{\partial x}\left(\frac{\partial^{2} A_{M}^{\prime}}{\partial x^{2}}-\frac{m^{2} A_{M}^{\prime}}{N^{2}}\right) \frac{m}{N}-A_{M}^{\prime}\left(\frac{\partial^{3} A_{M}^{\prime}}{\partial x^{3}}-\frac{m^{2}}{N^{2}} \frac{\partial A_{M}^{\prime}}{\partial x}\right) \frac{m}{N}\right\} \frac{1}{2} \sin \frac{2 m y}{N}
$$

The $\mathrm{x}$ average of Eq. 16 vanishes as

$$
\begin{gathered}
\frac{\partial A_{M}^{\prime}}{\partial x} \frac{\partial^{2} A_{M}^{\prime}}{\partial x^{2}}=\frac{1}{2} \frac{\partial\left(\partial A_{M}^{\prime} / \partial x\right)^{2}}{\partial x} \\
A_{M}^{\prime} \frac{\partial A_{M}^{\prime}}{\partial x}=\frac{1}{2} \frac{\partial\left(A_{M}^{\prime}\right)^{2}}{\partial x} \\
A_{M}^{\prime} \frac{\partial^{3} A_{M}^{\prime}}{\partial x^{3}}=\frac{\partial}{\partial x}\left(A_{M}^{\prime} \frac{\partial^{2} A_{M}^{\prime}}{\partial x^{2}}\right)-\frac{\partial A_{M}^{\prime}}{\partial x} \frac{\partial^{2} A_{M}^{\prime}}{\partial x^{2}}
\end{gathered}
$$


and

$$
\overline{\frac{\partial()}{\partial x}}^{x}=0
$$

The same is true for the Jacobian of the $\sin \frac{l y}{N}$ and the $\sin \frac{l y}{N}$ terms. On the other hand, the $\mathrm{x}$ averages of the Jacobians of the $\sin \frac{m y}{N}$ and the $\sin \frac{l y}{N}$ terms generally do not vanish and produce nonzero terms of the form $\overline{()} \sin \frac{(l-m) y}{N}$ and $\overline{()} \sin \frac{(l+m) y}{N}$. When projected onto the zonal mean base functions of the form $\overline{()} \cos \frac{k y}{N}$, we note that

$$
\int_{0}^{N \pi} \sin \frac{i y}{N} \cos \frac{j y}{N} d y=\left\{\begin{array}{cc}
0 & i-j \text { is even } \\
\frac{2 N i}{i^{2}-j^{2}} & i-j \text { is odd }
\end{array}\right.
$$

We also note that only changes in zonal mean components with odd k's change the total momentum. Therefore, if $\mathrm{l}-\mathrm{m}$ is odd, the Jacobian terms have zero projection onto those components, and the momentum is automatically conserved. If $1-\mathrm{m}$ is even, the Jacobian terms have non-zero projection onto zonal mean components with odd k's. Total momentum would not be conserved unless all zonal mean components have zero momentum when integrated over the whole channel.

\section{References}

[1] W. J. Randel and J. L. Stanford, "The observed life cycle of a baroclinic instability," J. Atmos. Sci. 42, 1364 (1985).

[2] A. J. Simmons and B. J. Hoskins, "The life cycles of some non-linear baroclinic waves," J. Atmos. Sci. 35, 414 (1978).

[3] S. B. Feldstein and I. M. Held, "Barotropic decay of baroclinic waves in a two-layer beta-plane model," J. Atmos. Sci. 46, 3416 (1989).

[4] E. K. M. Chang, "Downstream development of baroclinic waves as inferred from regression analysis," J. Atmos. Sci. 50, 2038 (1993).

[5] S. Lee and I. M. Held, "Baroclinic wave packets in models and observations," J. Atmos. Sci. 50, 1413 (1993).

[6] J. Pedlosky, Geophysicsl Fluid Dynamics, 2nd ed. (Springer-Verlag, New York, 1987).

[7] J. G. Esler, "Wave packets in simple equilibrated baroclinic systems," J. Atmos. Sci. 54, 2820 (1997). 


\title{
Can a Simple Two-Layer Model Capture the Structure of Easterly Waves?
}

\author{
Cheryl L. Lacotta
}

\section{Introduction}

Most tropical storms in the Atlantic, and even many in the eastern Pacific, are due to disturbances caused by African easterly waves. In fact, Avila [1] claims all of the 20 storms of the 1990 eastern North Pacific hurricane season can be traced back to waves originating over Africa. This connection to Africa is not always obvious as the waves seem to weaken on the eastern coast of Central America and then regenerate in the Pacific. Whether or not these disturbances propagate from Africa or develop in situ, the growth stage in the Pacific can often lead to a breakdown of the intertropical convergence zone (ITCZ), which in turn leads to cyclogenesis [2]. To investigate the growth of these waves in the Pacific, it would be useful to examine the progression of easterly waves from the Atlantic Ocean, over the North American continent, and into the Pacific Ocean. Unfortunately, a large data void in the eastern Pacific makes such an investigation difficult.

Another approach is to study these waves using a mathematical model. This paper proposes to simulate the structure of easterly waves using the simplest model possible: a two layer, quasi-geostrophic, linearized model. In this way, we may analyze the wave characteristics to examine the behavior of the wave as it passes from the Atlantic to the Pacific Ocean.

The second section of this paper describes the known properties of easterly waves. The third section explains and derives the model used in this investigation. A complete look at the dispersion relationship for the model is contained in the fourth section. Finally, an explanation of the results and avenues for future work are presented.

\section{Characteristics of Easterly Waves}

African easterly waves are westward-propagating waves originating in northern Africa from mid-June to early October. According to Burpee [3], the preferred region of origin is between $5^{\circ} \mathrm{E}$ and $35^{\circ} \mathrm{E}$, near $15^{\circ} \mathrm{N}$. Based on satellite pictures and statistical methods, Burpee dismissed orographic forcing and afternoon convection as the origin of easterly waves. Instead, he deduced that the waves are caused by an instability of the mid-tropospheric easterly jet. Specifically, the horizontal and vertical shear of the mean zonal flow may be acting as sources of energy for the African easterly waves. Burpee showed that the jet satisfies 
the Charney and Stern [4] instability criterion, and that these waves are thus growing both barotropically and baroclinically.

Reed and Recker [5] sketched the complete structure of easterly waves from a statistical standpoint. Their results were based on a compositing technique which yielded average properties of the 18 disturbances they studied from stations within the area defined by $130^{\circ} \mathrm{E}$ to $175^{\circ} \mathrm{E}$, and $0^{\circ} \mathrm{N}$ to $20^{\circ} \mathrm{N}$. In [5, Fig. 3] (this, and all other figures from [5] are shown in the appendix), the wind profiles for three of these stations are shown. The stations at Koror and Majuro represent the western and eastern ends of the network, respectively. The profile for Koror shows westerly winds below $600 \mathrm{mb}$, and easterly winds above. At the eastern end, the winds are easterly at all heights, with wind speeds increasing with height. This figure also shows that the average wave velocity is westward at $9 \mathrm{~m} / \mathrm{s}$ in the eastern half of the region. In other words, they identified an interval of about $3800 \mathrm{~km}$ between successive ridge positions, with a ridge passing a station roughly every five days.

$[5$, Fig. 4] shows the composite diagram of the meridional wind speed with reference to the trough and ridge of the wave. There is a low-level maximum of northerly wind to the west of the trough, and a maximum of the southerly wind to the east. The centers of the maxima are located at about $700 \mathrm{mb}$. In comparison to these low-level winds, the upper winds are almost completely out of phase. The peak southerly winds in the upper layer are situated almost directly above the lower layer peak northerly winds, and vice versa. Vertically there is an eastward tilt between the centers of northerly and southerly wind maxima. In fact, it was observed that the maximum southerly wind at high levels lags the low-level northerly wind peak by approximately one-eighth of a wavelength.

A composite diagram of horizontal divergence [5, Fig. 8a] shows a region of convergence centered at the trough. The peak of this convergence region tilts eastward with height, just as did the maxima of the north and south winds in [5, Fig. 4]. Sitting atop this region is one of divergence, centered at about $175 \mathrm{mb}$. The altitude of this divergence suggests that it may be an effect of the spreading of cumulonimbus anvils. Consistent with the conservation of mass, Reed and Recker showed that low-level convergence forced rising motion above the trough in a composite diagram of vertical velocities [5, Fig. 8b].

The composite diagram of relative humidity [5, Fig. 6] also demonstrates the eastward tilt of the other figures. The moist air, because of the tilt, is found in advance of the trough at the lowest levels and to the rear at higher levels. The driest air follows the passage of the ridge. This is consistent with the rainfall plots of all the stations [5, Fig. 11]. When plotted as a function of wave position, the majority of the rain precedes or is coincident with the trough axis. When viewed in conjunction with [5, Fig. 8b], it appears that convection provides positive feedback to the rising motion above the trough.

\section{The Model}

This paper attempts to further the understanding of easterly waves through an investigation of the dispersion relationship for the two-layer, linearized, quasi-geostrophic Rossby wave equations. These equations were chosen because Holton [6] has shown that easterly waves may be theoretically interpreted as forced equatorial waves. The top and bottom layers are labelled Layer 1 and Layer 2, respectively. The equations are (after Salmon [7]): 


$$
\begin{gathered}
\left(\frac{\partial}{\partial t}+U \frac{\partial}{\partial x}\right)\left(\frac{\partial^{2} \psi_{1}}{\partial x^{2}}\right)+\beta \frac{\partial \psi_{1}}{\partial x}-\frac{f_{0}}{\delta p} \omega=0 \\
\left(\frac{\partial}{\partial t}-U \frac{\partial}{\partial x}\right)\left(\frac{\partial^{2} \psi_{2}}{\partial x^{2}}\right)+\beta \frac{\partial \psi_{2}}{\partial x}+\frac{f_{0}}{\delta p} \omega=0 \\
\frac{\partial}{\partial t}\left(\psi_{1}-\psi_{2}\right)-U \frac{\partial}{\partial x}\left(\psi_{1}+\psi_{2}\right)-\frac{R}{f_{0}} Q=\frac{\sigma \delta p}{f_{0}} \omega
\end{gathered}
$$

where $\mathrm{U}$ is the mean thermal wind, or wind shear, in the center of mass frame of reference, $\psi_{1}$ and $\psi_{2}$ are the corresponding streamfunctions for the top and bottom layers, $\beta=\frac{d f}{d y}$ where $f$ is the Coriolis parameter, $\delta p$ is the change in pressure from Layer 1 to Layer $2, R$ is the gas constant for dry air, and $Q$ is a heat source. In equation (3), $\sigma$ is the static stability parameter defined by $\sigma \equiv-R T_{0} p^{-1} d \ln \theta_{0} / d p$, where $\theta_{0}$ is the potential temperature corresponding to the basic state temperature $T_{0}$, and $\omega$ is the vertical velocity in pressure coordinates. With a few definitions,

$$
\lambda^{2}=\frac{f_{0}^{2}}{\sigma \delta p^{2}}, \quad \psi=\frac{\psi_{1}+\dot{\psi_{2}}}{2}, \quad \tau=\frac{\psi_{1}-\psi_{2}}{2}, \quad Q^{\prime}=\frac{R}{2 f_{0}} Q
$$

we may eliminate $\omega$ to create a system of two equations for the two variables, $\psi$ and $\tau$.

$$
\begin{gathered}
\frac{\partial}{\partial t}\left(\frac{\partial^{2} \psi}{\partial x^{2}}\right)+\beta \frac{\partial \psi}{\partial x}+U \frac{\partial}{\partial x}\left(\frac{\partial^{2} \tau}{\partial x^{2}}\right)=0 \\
\frac{\partial}{\partial t}\left(\frac{\partial^{2} \tau}{\partial x^{2}}\right)+\beta \frac{\partial \tau}{\partial x}+U \frac{\partial}{\partial x}\left(\frac{\partial^{2} \psi}{\partial x^{2}}\right)-2 \lambda^{2}\left(\frac{\partial \tau}{\partial t}+U \frac{\partial \psi}{\partial x}+Q^{\prime}\right)=0
\end{gathered}
$$

The heat source term is proportional to the lower-level streamfunction. Therefore we may write $Q^{\prime}=\alpha \psi_{2}=\alpha(\psi-\tau)$, where $\alpha$ is a complex constant. Specifically $\alpha$ is assumed to have the form $\alpha=|\alpha| e^{i \phi}$, where $|\alpha|$ is the magnitude of $\alpha$ and $\phi$ is the phase. Based on the observations of Reed and Recker, we may choose at what phase to add the heat source term. From [5, Fig. 11] we see that most of the rainfall and cloud cover occurs over the trough axis. That implies that there must be convection at or near the trough axis. In order to have convection, a parcel must be warmer than its environment. Therefore, we need to add the heat source at a point where the lower-level environment is coolest. This results in choosing that $Q^{\prime}$ be $180^{\circ}$ out of phase with the lower-level streamfunction, so $\alpha=|\alpha| e^{i \pi}$, or $\alpha=-|\alpha|$. For simplicity, I will write $Q^{\prime}=-\alpha(\psi-\tau)$.

Assuming solutions of the form

$$
\psi=A e^{i k(x-c t)}, \quad \tau=B e^{i k(x-c t)}
$$

and substituting into equations (5) and (6), this system can then be written in matrix form, which after some algebraic simplifications is given by:

$$
\left[\begin{array}{cc}
k^{2} c+\beta & -k^{2} U \\
\left(-k^{2}\right) U+2 \lambda^{2} U-i \frac{2 \lambda^{2} \alpha}{k} & k^{2} c+\beta+2 \lambda^{2}+i 2 \lambda^{2} \alpha
\end{array}\right]\left[\begin{array}{l}
A \\
B
\end{array}\right]=\mathbf{0}
$$


At this point it is useful to scale the system to a critical zonal velocity,

$$
U_{\text {crit }} \equiv \beta(\text { radius } \quad \text { of } \quad \text { deformation })^{2}=\frac{\beta}{2 \lambda^{2}} .
$$

Then we may introduce non-dimensional parameters according to this scaling:

$$
k_{*}=\frac{k}{\sqrt{2} \lambda}, \quad c_{*}=c \frac{2 \lambda^{2}}{\beta}, \quad U_{*}=U \frac{2 \lambda^{2}}{\beta}, \quad \alpha_{*}=\alpha \frac{\lambda}{\beta} .
$$

In non-dimensional terms, (8) becomes

$$
\left[\begin{array}{cc}
k_{*}^{2} c_{*}+1 & -k_{*}^{2} U_{*} \\
\left(-k_{*}^{2}\right) U_{*}+U_{*}-i \frac{\sqrt{2} \alpha_{*}}{k_{*}} & k_{*}^{2} c_{*}+c_{*}+1+i \frac{\sqrt{2} \alpha_{*}}{k_{*}}
\end{array}\right]\left[\begin{array}{c}
A \\
B
\end{array}\right]=\mathbf{0}
$$

In order to find nontrivial solutions to the system, we must set the determinant of (9) to zero,

$$
\left|\begin{array}{cc}
k_{*}^{2} c_{*}+1 & -k_{*}^{2} U_{*} \\
\left(-k_{*}^{2}\right) U_{*}+U_{*}-i \frac{\sqrt{2} \alpha_{*}}{k_{*}} & k_{*}^{2} c_{*}+c_{*}+1+i \frac{\sqrt{2} \alpha_{*}}{k_{*}}
\end{array}\right|=0
$$

With some algebra (and dropping the ${ }_{*}$ for simplicity), we find that the dispersion relationship is:

$$
\begin{aligned}
c= & -\frac{2 k^{2}+i \sqrt{2} k \alpha+1}{2\left(k^{4}+k^{2}\right)} \pm \\
& {\left[\frac{\left(2 k^{2}+i \sqrt{2} k \alpha+1\right)^{2}-4\left(k^{4}-k^{2}\right)\left(1+\frac{i \sqrt{2} \alpha}{k}+U^{2} k^{2}\left(1-k^{2}\right)-i \sqrt{2} U \alpha k\right)}{4\left(k^{4}+k^{2}\right)^{2}}\right]^{\frac{1}{2}} }
\end{aligned}
$$

\section{The Dispersion Relationship}

A dispersion relationship determines the phase speed of waves of a given wavenumber. Now that we have this relationship for the model, we may investigate some special cases.

\subsection{Case 1: No Heat Added}

The case where there is no heating in the system provides a good check for the work done thus far. Without the $\alpha$ terms in (10), this case is simply the classic baroclinic instability problem. On axes of the non-dimensional wind shear and wave number, contours of the imaginary part of the phase speed, c, defined by:

$$
c=\frac{-2 k^{2}-1 \pm\left(1+4 k^{8} U^{2}-4 k^{4} U^{2}\right)^{\frac{1}{2}}}{2 k^{2}\left(k^{2}+1\right)}
$$

are plotted in Fig. 1. Shown in the plot are the contours of maximum $\Im(c)$ of the two modes. The contours of $\Im(c)$ are all positive values, implying that the wave amplitude grows for values of $\mathrm{k}$ and $\mathrm{U}$ in the contoured region. The typical textbook discussion of 


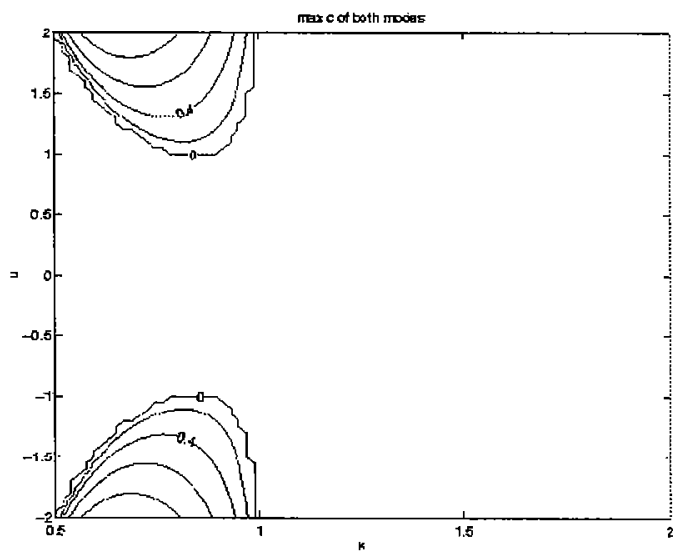

Figure 1: Contours of the imaginary part of the phase speed. No heat is added to the system.

baroclinic instability, such as that in Holton [7] shows only the upper left region of this plot. The wind shear, U, only appears as a quadratic in (12). Therefore values of $\Im(c)$ will be symmetric across the axis, $U=0$. In other words, the growth of the wave has no dependence on the sign of the wind shear.

\subsection{Case 2: Heat Added, No Wind Shear}

As another check for the model, we consider the case in which there is no wind shear, $U=0$. The heat has been added as described above. Solving for $\mathrm{c}$ under these conditions, we find two solutions,

$$
c_{1}=-\frac{1}{k^{2}}, \quad c_{2}=\frac{-\left(1-\frac{i \sqrt{2} \alpha}{k}\right)}{k^{2}+1},
$$

corresponding to the two possible modes of oscillation. The phase speed $c_{1}$ is simply the dispersion relationship for barotropic Rossby waves. The baroclinic mode, given by $c_{2}$, shows wave growth for all wavenumbers. In other words, $\Im\left(c_{2}\right)>0$ for all $k$. See Fig. 2 .

The non-dimensional heating term, $\alpha$, is given the value $\alpha=-1$ for this and future calculations. This was found using the scaling argument:

$$
\alpha_{*}=\alpha \frac{\lambda}{\beta}=\frac{R Q^{\prime}}{2 f_{0} \psi_{2}} \frac{k}{k} \frac{\lambda}{\beta}
$$

This is simply the definition of $\alpha$ multiplied by its scaling factor. Here, $R$ is the gas constant, $Q^{\prime}$ is the heating rate, observed to be about $4^{\circ} \mathrm{C}$ /day in the tropics, $f_{0}$ is the Coriolis parameter at about $15^{\circ} \mathrm{N}, k$ is the preferred wavenumber for easterly waves, $\lambda$ is the reciprocal of the radius of deformation, and $\beta=\frac{d f}{d y}$ near $15^{\circ} \mathrm{N}$. The quantity $\left(\psi_{2} k\right)$ is merely the meridional wind speed in the lower layer, observed to be about $3 \mathrm{~m} / \mathrm{s}$. When these values are applied to (13), keeping the units consistent, we find $\alpha \sim 5$. Therefore, 

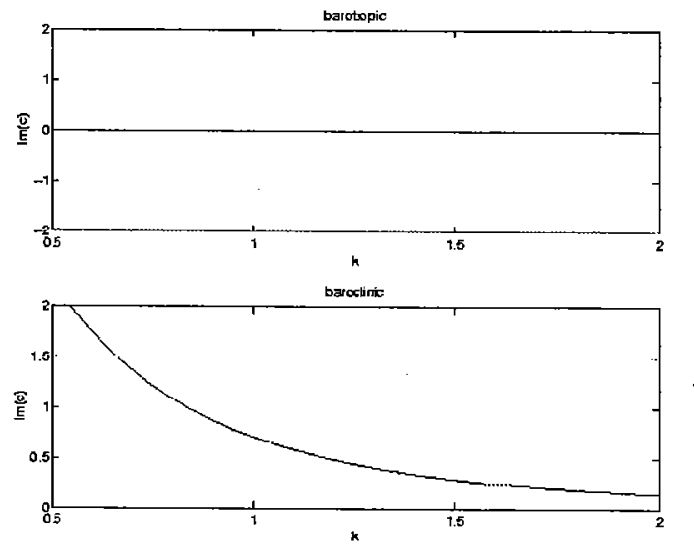

Figure 2: The imaginary part of the phase speed plotted against wavenumber for the two modes, barotropic and baroclinic, in the case without wind shear. $\alpha=-1$

$\alpha$ is $O(1)$ and must not be neglected in comparison to the other terms in (11). To be conservative, in this model we choose $|\alpha|=1$, and considering the phase, we get $\alpha=-1$.

\subsection{Case 3: Wind Shear and Heat Included}

Shown in Fig. 3 are the contours of $\Im(c)$, where $c$ is defined by equation (11).

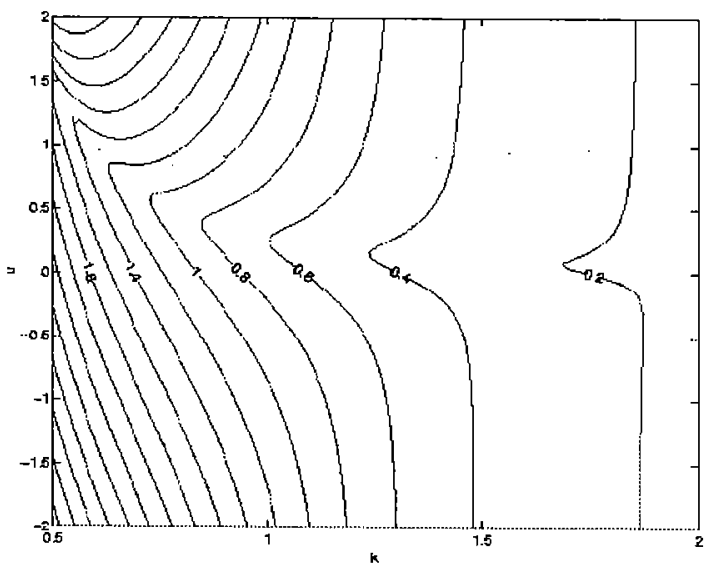

Figure 3: Contours of the imaginary part of the phase speed. Shear and heat are added.

Now, because of the heat term, the contours are no longer symmetric across $U=0$. The shear term no longer appears only as a quadratic, forcing the sign of the shear to be important. Without the heat source, there are only two regions where instabilities would occur. Outside of these regions the waves are stable and will not grow. Now that heat has been added, it is clear from Figure 3 that there are no longer any stable regions. The 
contours of $\Im(c)$ are positive, or unstable, everywhere.

For each of the two modes, the real and imaginary parts of the phase speed have been plotted for a particular wavenumber. Recall that the non-dimensional wavenumber is defined:

$$
k_{*}=k(\text { radius } \quad \text { of } \quad \text { deformation })=\frac{k}{\sqrt{2} \lambda}
$$

Since $k=2 \pi /$ wavelength by definition, we need to determine the typical wavelength of the easterly waves. Using Reed and Recker's results, we approximate the wavelength as 4000 $\mathrm{km}$. With a typical radius of deformation of $1000 \mathrm{~km}$ for tropical waves, we find:

$$
k_{*}=\frac{2 \pi}{4000} \cdot 1000 \approx \frac{3}{2}
$$

Figures 4 and 5 show the real and imaginary parts of the phase speed plotted against the wind shear for $k=1.5$.

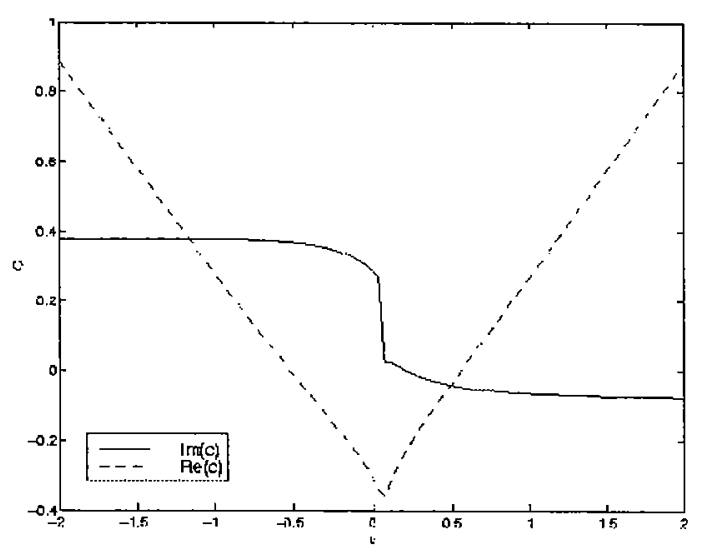

Figure 4: Contours of the real and imaginary parts of the phase speed of Mode 1 . The contours are calculated for $k=1.5$ and $\alpha=-1$.

Looking at the real part of the phase speed for Mode 1 (Fig. 4), we see that for small shear $(|U|<0.5), \Re(c)<0$, implying that the wave is easterly. However, if the shear increases, the wave becomes westerly. For this mode the wave will grow with a negative wind shear since $\Im(c)>0$ for $U<0$. By Fig. 5, however, we see that the wave will be easterly regardless of the shear in Mode 2. This is because $\Re(c)<0$ for all $U$. In this mode, the wave will grow only for positive shear. Because westward propagation (characteristic of easterly waves) is independent of the wind shear in Mode 2, it seems reasonable to claim that this mode is most representative of the easterly waves studied by Reed and Recker.

To investigate this case in more detail, we will look at specific values of shear. Recall that $U$ is defined as

$$
U=\frac{U_{1}-U_{2}}{2}
$$




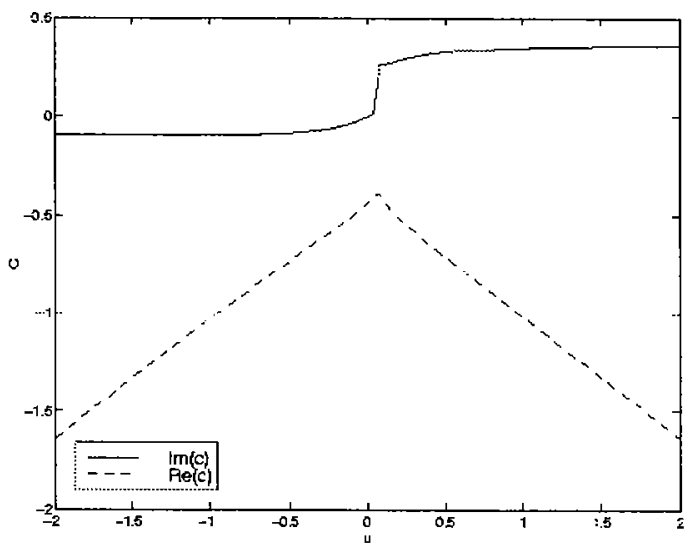

Figure 5: As in Fig. 4, for Mode 2.

where $U_{1}$ and $U_{2}$ are the zonal wind speeds for the top and bottom layers, respectively. Also recall that $U$ was non-dimensionalized by

$$
U_{*}=\frac{U}{U_{c r i t}}=U \frac{2 \lambda^{2}}{\beta}
$$

Taking $U$ to be about $2 \mathrm{~m} / \mathrm{s}$ (from [5, Fig. 3]), and $U_{\text {crit }}$ to be about $10 \mathrm{~m} / \mathrm{s}$, we find that $U_{*} \approx 0.2 \mathrm{~m} / \mathrm{s}$. Again, to be conservative in the model, $U= \pm 0.1$ was used. Referring back to Eq. (7), we set $B=1$ so that

$$
\tau=e^{i k x}, \quad \psi=\frac{k^{2} U}{1+k^{2} c} e^{i k x}
$$

where $c$ is complex. Now, using the definitions in (4), we may find the streamfunctions for the upper and lower layers. The streamfunctions for the mode with maximum growth in the case of negative shear (i.e. Mode 1 from above) are plotted in Fig. 6. The figure shows that the upper layer streamfunction is leading the lower level one by about one-eighth of the wavelength. Although the displacement between the two streamfunctions is what was observed by Reed and Recker [5], the relative placement of the two are reversed. Recall in [5, Fig. 4] that the trough axis has an eastward tilt. In other words, the lower level streamfunction should lead the upper level one.

For comparison, we now do the same analysis with the mode which has maximum growth with a positive shear, i.e. $U=+0.1$. The results are plotted in Fig. 7 below. Now we see that not only do we have the same displacement of one-eighth of the wavelength, but we also have the eastward tilt in the trough axis, comparable to observations of easterly waves. Once again, it appears that Mode 2 from the discussion above is representative of easterly waves. 


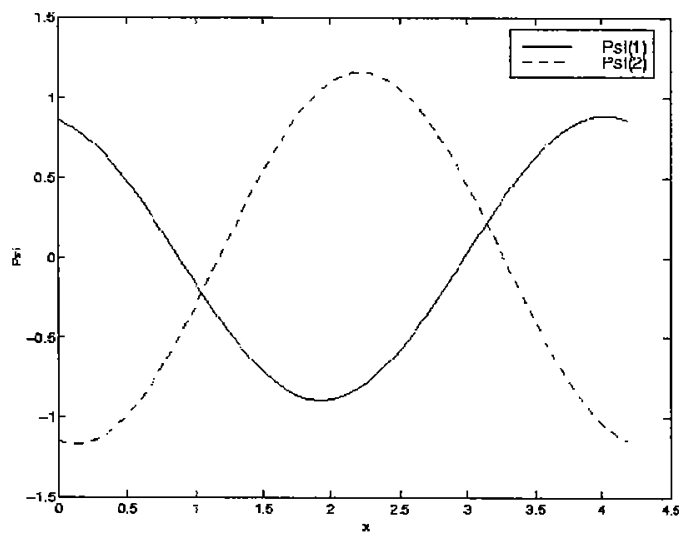

Figure 6: The upper and lower streamfunctions, $\psi_{1}$ and $\psi_{2}$ respectively, for the case of $U=-0.1$ and $k=1.5$.

\section{Conclusion}

This project was developed to investigate the progression of easterly waves from the Atlantic Ocean to the East Pacific Ocean. The idea was to try to explain the wave behavior in view of a pseudomomentum argument as the wave passes the North American continent, similar to that of Held [8]. Originally, the project was to develop the relationship between the upper and lower streamfunctions, and then apply this information to the pseudomomentum theory.

The results of the model runs show that one can find a mode in the two-layer, linearized, quasi-geostrophic model that simulates the structure of easterly waves as observed in the West Pacific by Reed and Recker. The model shows an eastward tilt in the trough axis. In other words, the upper level lags the lower level. Further, the displacement between the two levels is roughly one-eighth the wavelength of the easterly wave. A more detailed analysis must be done to compare the wave speed and doubling time with observations.

In order to use this study to continue with the original research, it must be verified that the model also simulates easterly waves in the East Pacific or Atlantic Oceans. Reed and Recker's study on easterlies in the West Pacific was used only because it provided information on the complete structure of the easterlies. However, Holton [6] indicates that the shear may differ from one side of the Pacific to another. Indeed, there is variability in the shear, as evidenced by [5, Fig. 3] as one moves across the Pacific. To what extent this will affect the model has yet to be determined. Once this is known, attention may then be turned to the question of whether or not the wave behavior may be modified by pseudomomentum arguments. This is the plan for future work.

Acknowledgments I would like to thank the wonderful staff of the GFD 2000 Program, particularly my advisors for the summer: Wayne Schubert, Isaac Held, and Rick Salmon. I would also like to thank the fellows for their help, friendship, and a wonderful summer. 


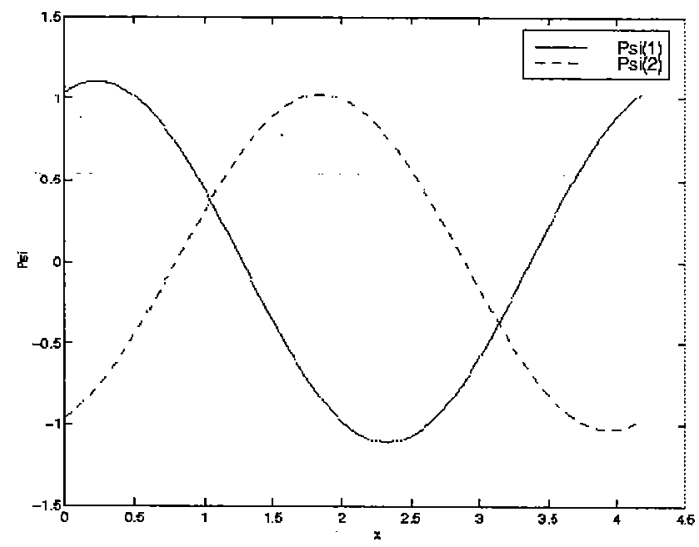

Figure 7: As in Fig. 6, for the case of $U=+0.1$ and $k=1.5$.

\section{References}

[1] L. A. Avila, "Eastern north pacific hurricane season of 1990," Mon. Wea. Rev. 119, 2034 (1991).

[2] R. N. Ferreira and W. H. Schubert, "Barotropic aspects of ITCZ breakdown," J. Atmos. Sci. 54, 261 (1997).

[3] R. W. Burpee, "The origin and structure of easterly waves in the lower troposphere of north africa," J. Atmos. Sci. 29, 77 (1972).

[4] J. G. Charney and M. E. Stern, "On the stability of internal baroclinic jets in a rotating atmosphere," J. Atmos. Sci. 19, 159 (1962).

[5] R. J. Reed and E. E. Recker, "Structure and properties of synoptic-scale wave disturbances in the equatorial western pacific," J. Atmos. Sci. 28, 1117 (1971).

[6] J. R. Holton, "A diagnostic model for equatorial wave disturbances: The role of vertical shear of the mean zonal wind," J. Atmos. Sci. 28, 55 (1971).

[7] R. Salmon, Lectures On Geophysical Fluid Dynamics (Oxford University Press, New York, 1998).

[8] I. M. Held and R. T. Pierrehumbert, "Dissipative destabilization of external rossby waves," J. Atmos. Sci. 43, 388 (1986). 


\section{Appendix}

The following are figures taken from Reed and Recker [5].

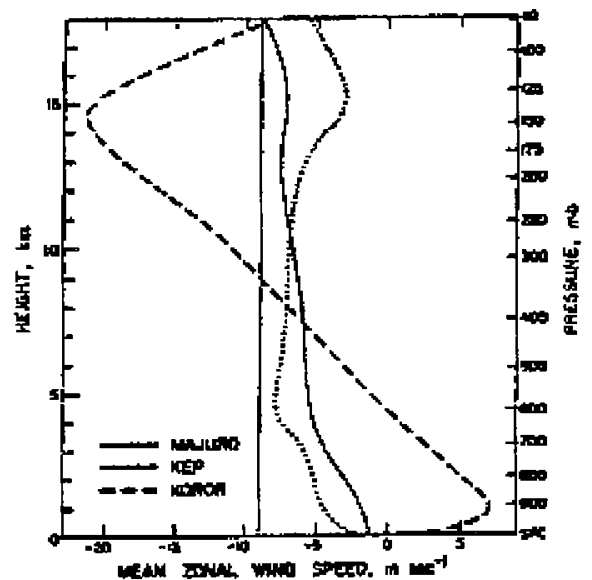

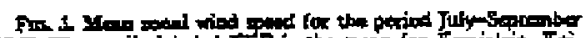

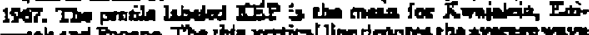

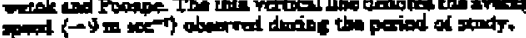

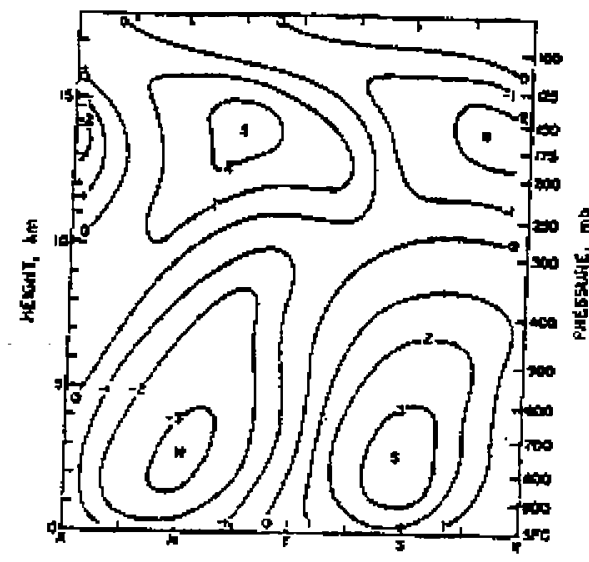

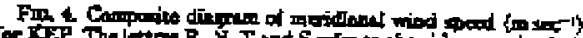

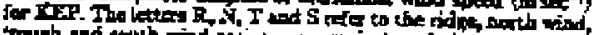

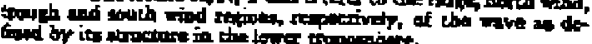

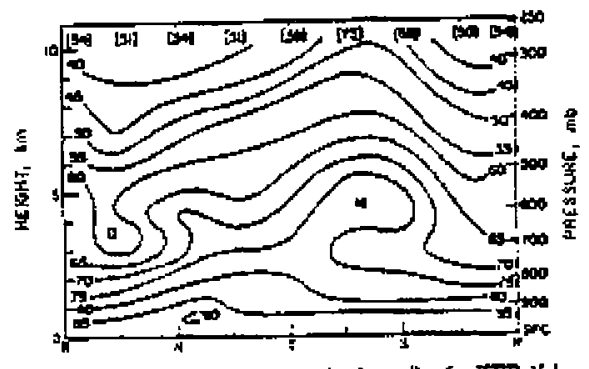

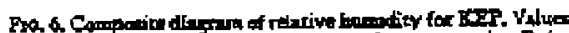

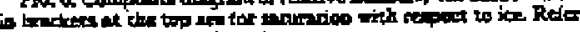

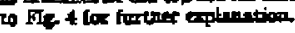



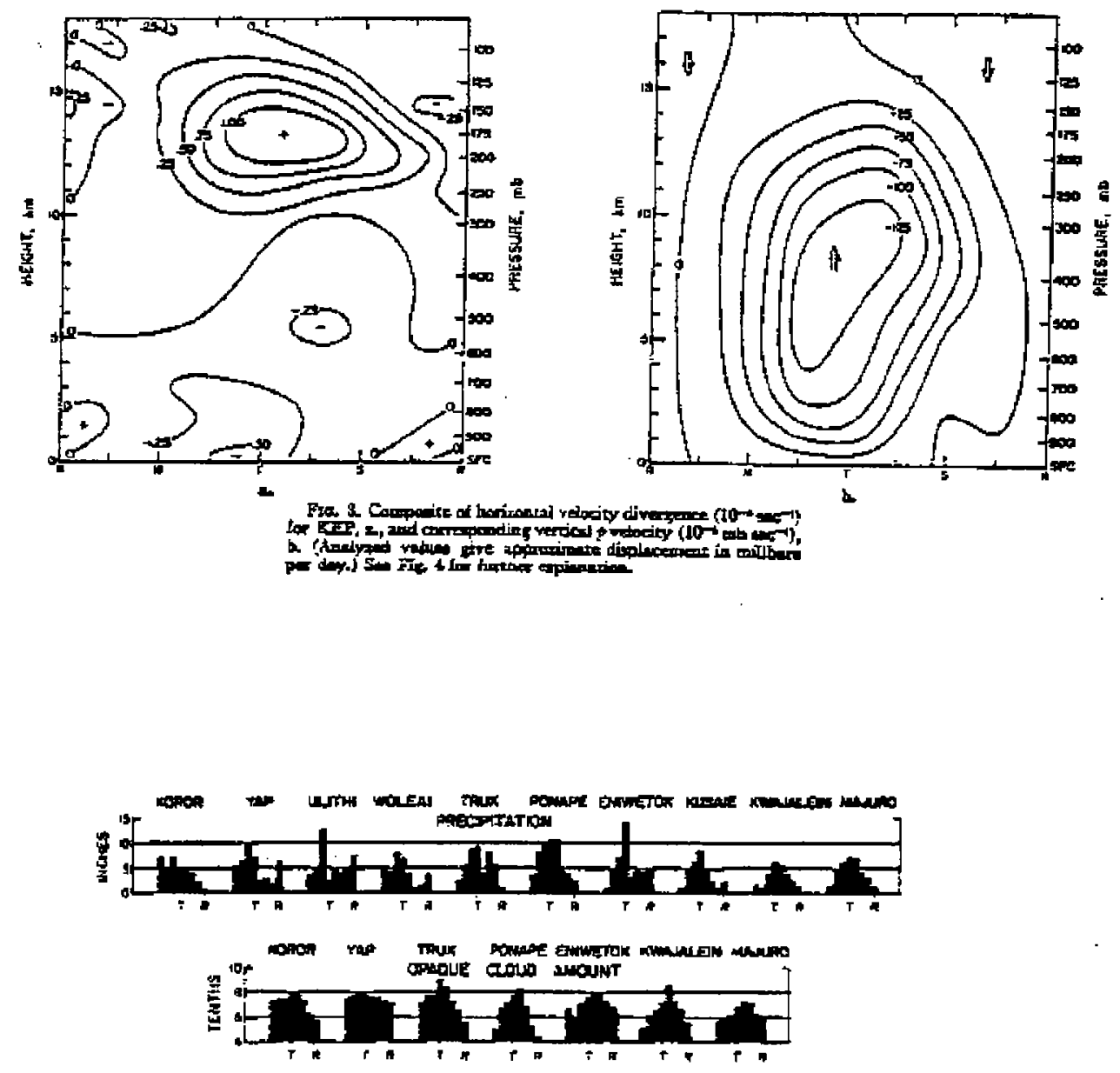

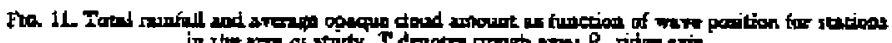

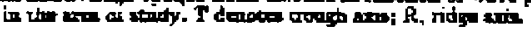




\title{
Eddy PV Fluxes in a One Dimensional Model of Quasi-Geostrophic Turbulence
}

\author{
Christos M.Mitas
}

\section{Introduction}

\subsection{Motivation}

Understanding eddy transport of heat and momentum is crucial to developing closure schemes for general circulation models.

While eddy momentum fluxes do not adhere to a clear parameterization methodology, potential vorticity (PV) and heat fluxes may be easier to comprehend and thus parameterize.

If the eddy diffusivity (defined as the eddy flux over the mean gradient) was computed from a simple homogeneous model, it could be fit with an analytic function of the various model parameters, and subsequently used in a inhomogeneous model to provide closure for the zonal mean quantities that are forced by eddy fluxes.

\subsection{Problem Formulation}

Highly idealized baroclinic models have been frequently used to study various aspects of homogeneous turbulence $([1,2,3,4])$. These studies used doubly periodic, two-layer, quasi geostrophic models on a $\beta$ plane to represent the interior of a channel flow that is continuously forced by an imposed vertical shear $(U)$. The use of a doubly periodic box is enforcing the homogeneity of the turbulent flow. In both [3] and [4] the eddy statistics of the equilibrated flow do not appear to depend on the length of the domain, thus the representation of the channel interior seems to be accurate.

To achieve computational economy and at the same time retain essential features of homogeneous turbulence, [4] in their study of baroclinic eddy fluxes have used a truncated model. This model retains only the zonally averaged flow and one nonzero wavenumber in the zonal direction, but has high meridional resolution to allow for an enstrophy cascade. Severely truncated models have been used in numerous meteorological studies, for diverse topics as sudden stratospheric warmings ([5]) and climate sensitivity ([6]), among others.

In this project a doubly periodic, quasi geostrophic, two-layer model, with a rigid lid and flat bottom, truncated in the zonal direction and with high resolution in the meridional direction is implemented and used to assess eddy fluxes. 


\section{Methodology}

\subsection{The Two Layers, Doubly Periodic, QG Model}

\subsubsection{Equations}

In the formulation of the model we follow [4] . The layers are of equal depth $H$. A rigid lid on top and a bottom horizontal surface defines the vertical boundaries. The Coriolis parameter is $f_{0}$. The meridional coordinate $y$ measures distance from the domain center. Subscripts 1 and 2 refer to the upper and lower layer, respectively. The internal Rossby radius of deformation is defined by $\lambda^{2}=g\left(\rho_{2}-\rho_{1}\right) H /\left(2 \rho_{2} f_{0}^{2}\right)$. Ekman friction is implemented only for the lower layer. A scale-selective biharmonic diffusion of potential vorticity is included to absorb the enstrophy cascade. The friction and diffusion coefficients are denoted by $\kappa$ and $\nu$ respectively.

The dimensional equations in terms of the streamfunctions $\Psi_{j}$ read:

$$
\begin{gathered}
\frac{\partial Q_{1}}{\partial t}+J\left(\Psi_{1}, Q_{1}\right)=-\nu \nabla^{4} Q_{1} \\
\frac{\partial Q_{2}}{\partial t}+J\left(\Psi_{2}, Q_{2}\right)=-\nu \nabla^{4} Q_{2}-\kappa \nabla^{2} \Psi_{2}
\end{gathered}
$$

where the potential vorticity $Q_{j}$ is:

$$
Q_{j}=\beta y+\nabla^{2} \Psi_{j}+(-1)^{j}\left(\Psi_{1}-\Psi_{2}\right) /\left(2 \lambda^{2}\right), \quad j=1,2
$$

We now assume that there is a positive vertical shear $U$ and that the upper and lower layer streamfunctions are given by:

$$
\begin{aligned}
& \Psi_{1}(x, y, t)=-\frac{U}{2} y+\psi_{1}(x, y, t) . \\
& \Psi_{2}(x, y, t)=+\frac{U}{2} y+\psi_{2}(x, y, t)
\end{aligned}
$$

We non-dimensionalize $\left(x, y, t, \psi_{j}\right)$ with $(\lambda, \lambda, \lambda / U, U \lambda)$. It follows that the dimensionless parameters are $\hat{\beta}=\beta \lambda^{2} / U, \hat{\kappa}=\kappa \lambda / U$, and $\hat{\nu}=\nu /\left(U \lambda^{3}\right)$.

If we drop the hats in the dimensionless parameters the eddy equations become:

$$
\frac{\partial q_{j}}{\partial t}+J\left(\psi_{j}, q_{j}\right)=F_{j}+D_{j}
$$

The transient eddy potential vorticities are given by:

$$
q_{j}=\nabla^{2} \psi_{j}+(-1)^{j} \tau
$$

where $\tau=\left(\psi_{1}-\psi_{2}\right) / 2$ is the baroclinic perturbation that is proportional to the vertical thickness. The right-hand-side terms represent forcing by the imposed mean flow,

$$
F_{1}=-\frac{1}{2} \frac{\partial q_{1}}{\partial x}-(\beta+1 / 2) \frac{\partial \psi_{1}}{\partial x}
$$




$$
F_{2}=+\frac{1}{2} \frac{\partial q_{2}}{\partial x}-(\beta-1 / 2) \frac{\partial \psi_{2}}{\partial x}
$$

and dissipation by Ekman friction and hyper-diffusion:

$$
\begin{gathered}
D_{1}=-\nu \nabla^{4} q_{1} \\
D_{2}=-\nu \nabla^{4} q_{2}-\kappa \nabla^{2} \psi_{2}
\end{gathered}
$$

To retain only the zonal mean and a nonzero wavenumber $k$ in the $\mathrm{x}$-direction, we assume that the streamfunctions $\psi_{j}$ are of the form:

$$
\psi_{j}(x, y, t)=\psi_{j, 0}(y, t)+\left(\psi_{j, k}(y, t) e^{i k x}+c . c .\right)
$$

where the $\psi_{j, 0}(y, t)$ is referred as the "zonal mean component" and the $\psi_{j, k}(y, t)$ as the "wave component". If we further ignore wave-wave interactions (i.e. the $e^{2 i k x}$ waves ), the resulting equations for the two components are:

$$
\begin{gathered}
\frac{\partial q_{j, 0}}{\partial t}=-2 \operatorname{Re} \frac{\partial\left(i k \psi_{j, k} q_{j, k}^{*}\right)}{\partial y} \\
\frac{\partial q_{j, k}}{\partial t}=i k\left(q_{j, k} \frac{\partial \psi_{j, 0}}{\partial y}-\psi_{j, k} \frac{\partial q_{j, 0}}{\partial y}\right)
\end{gathered}
$$

where an asterisk denotes complex conjugation, and the decomposition of $q_{j}, F$ and $D$ is as in (4).

\subsubsection{Numerical Solution}

We choose to solve the real equation (5), and complex equation (6) using spatial finite differencing and a leapfrog scheme for the time differencing. Inspection of the equations suggest that the zonal mean and wave components should be staggered with respect to each other so that the $y$-derivatives would be computed with the smallest error. Note that with this type of staggering, the non-linear terms, corresponding to the Jacobians in equation (3) exactly conserve enstrophy and energy.

$\hat{L}$ is the non-dimensional parameter controlling the length of the domain that is set to $2 \pi \hat{L}$. All the experiments reported here have used $\hat{L}=10$. The number of grid points in the $y$-direction is set to $N=256$. With this choice for $\hat{L}$ and $N$, the meridional wavenumber is given by: $l_{n}=n / L, n=1 \cdots N / 2$, where $n$ is the discrete meridional wavenumber. Thus, the grid spacing is $d y=\sim 0.245$ (i.e. $\sim 4$ points per internal radius of deformation), which appears to be adequate to resolve baroclinic eddies in our model.

\subsubsection{Spectra}

Following [4] again, we calculate spectra for the various quantities in our model (enstrophy $q_{j}^{2}$, kinetic energy $\frac{1}{2}\left(u_{j}^{2}+v_{j}^{2}\right)$, potential energy $\frac{1}{2} \tau^{2}$, etc. $)$ as follows: If the Fourier coefficients of the zonal mean components of two functions $f, g$ are $F_{0, n}, G_{0, n}$ and those of their wave components are $F_{k, n}, G_{k, n}$, then the spectrum $\left\{z_{n}\right\}$ of zonal component of $\langle f g\rangle$ is defined as:

$$
z_{n}=2 c_{n} \operatorname{Re}\left(F_{0, n} G_{0, n}^{*}\right), \quad n=1, \cdots, N
$$


and the spectrum $\left\{w_{n}\right\}$ of the wave component as:

$$
w_{n}=2 c_{n} \operatorname{Re}\left(F_{k, n} G_{k, n}^{*}+F_{k,-n} G_{k,-n}^{*}\right), \quad n=0, \cdots, N
$$

where $c_{n}$ is $\frac{1}{2}$, if $n=0$ or $n=N$, and is 1 , otherwise.

\subsection{Modal Variables Formulation}

An alternative representation of the two layers system may be formulated in terms of the so-called modal variables defined as: $\psi=\left(\psi_{1}+\psi_{2}\right) / 2$ and $\tau=\left(\psi_{1}-\psi_{2}\right) / 2$, where $\psi$ and $\tau$ are identified as the barotropic and baroclinic streamfunctions, respectively.

The non-dimensional equations are modified as follows:

$$
\begin{gathered}
\left.\left.\frac{\partial \nabla^{2} \psi}{\partial t}+J\left(\psi, \nabla^{2} \psi\right)\right)+J\left(\tau, \nabla^{2} \tau\right)\right)=-\frac{1}{2} \frac{\partial \nabla^{2} \tau}{\partial x}-\beta \frac{\partial \psi}{\partial x}-\frac{\kappa}{2} \nabla^{2}(\psi-\tau)-\nu \nabla^{4}\left(\nabla^{2} \psi\right) \\
\left.\left.\left\{\frac{\partial \nabla^{2} \tau}{\partial t}+J\left(\psi, \nabla^{2} \tau\right)\right)+J\left(\tau, \nabla^{2} \psi\right)\right)\right\}-\frac{\partial \tau}{\partial t}-J(\psi, \tau)= \\
\left\{-\frac{1}{2} \frac{\partial \nabla^{2} \psi}{\partial x}-\beta \frac{\partial \tau}{\partial x}-\frac{\kappa}{2} \nabla^{2}(\tau-\psi)-\nu \nabla^{4}\left(\nabla^{2} \tau\right)\right\}-\frac{1}{2} \frac{\partial \psi}{\partial x}+\nu \nabla^{4} \tau
\end{gathered}
$$

The corresponding dimensional equations on an $f$-plane may be found in [7].

For ease of reference we define barotropic and baroclinic vorticities as $\zeta=\nabla^{2} \psi, \phi=\nabla^{2} \tau$, respectively, and the baroclinic potential vorticity as $\rho=\nabla^{2} \tau-\tau=\phi-\tau$. Note that the barotropic vorticity and potential vorticity are identical.

The equations for the barotropic and baroclinic zonal mean components become:

$$
\begin{aligned}
\frac{\partial \zeta_{0}}{\partial t}= & -2 \frac{\partial R e\left(i k\left(\psi_{k} \zeta_{k}^{*}+\tau_{k} \phi_{k}^{*}\right)\right)}{\partial y} \\
& -\frac{\kappa}{2}\left(\zeta_{0}-\phi_{0}\right) \\
& -\nu \nabla^{4} \zeta_{0} \\
\frac{\partial \rho_{0}}{\partial t}= & \left\{-2 \frac{\partial \operatorname{Re}\left(i k\left(\psi_{k} \phi_{k}^{*}+\tau_{k} \zeta_{k}^{*}\right)\right)}{\partial y}\right\}+2 \frac{\partial \operatorname{Re}\left(i k\left(\psi_{k} \tau_{k}^{*}\right)\right)}{\partial y} \\
& \left\{-\frac{\kappa}{2}\left(\phi_{0}-\zeta_{0}\right)\right\} \\
& -\nu \nabla^{4}\left(\left\{\phi_{0}\right\}-\tau_{0}\right)
\end{aligned}
$$

The equations for the barotropic and baroclinic wave components read:

$$
\begin{aligned}
\frac{\partial \zeta_{k}}{\partial t}= & -i k\left(\psi_{k} \frac{\partial \zeta_{0}}{\partial y}-\zeta_{k} \frac{\partial \psi_{0}}{\partial y}+\tau_{k} \frac{\partial \phi_{0}}{\partial y}-\phi_{k} \frac{\partial \tau_{0}}{\partial)} y\right. \\
& -i k\left(\frac{1}{2} \phi_{k}+\beta \phi_{k}\right) \\
& -\frac{\kappa}{2}\left(\zeta_{k}-\phi_{k}\right) \\
& -\nu \nabla^{4}\left(\zeta_{k} e^{i k x}\right)
\end{aligned}
$$




$$
\begin{aligned}
\frac{\partial \rho_{k}}{\partial t}= & \left\{-i k\left(\psi_{k} \frac{\partial \phi_{0}}{\partial y}-\phi_{k} \frac{\partial \psi_{0}}{\partial y}+\tau_{k} \frac{\partial \zeta_{0}}{\partial y}-\zeta_{k} \frac{\partial \tau_{0}}{\partial y}\right\}+i k\left(\psi_{k} \frac{\partial \tau_{0}}{\partial y}-\tau_{k} \frac{\partial \psi_{0}}{\partial y}\right)\right. \\
& \left\{-i k\left(\frac{1}{2} \zeta_{k}+\beta \tau_{k}\right)\right\}-\frac{1}{2} i k \psi_{k} \\
& \left\{-\frac{\kappa}{2}\left(\phi_{k}-\zeta_{k}\right)\right\} \\
& -\nu \nabla^{4}\left(\left(\left\{\phi_{k}\right\}-\tau_{k}\right) e^{i k x}\right)
\end{aligned}
$$

Note that the modal formulation is exactly equivalent in terms of truncation, domain size, numerics, conservation laws, and non-dimensionalization to the two-layer formulation. The reason for the re-formulation of the model will be discussed in the next section when we describe a reduced model derived from the modal variables model.

The non-dimensional parameters of the model are still $\beta$ (which may be thought as the inverse of supercriticality $\xi=\left(U / \lambda^{2}\right) / \beta$ ) and the frictional parameter $\kappa$. Inviscid instability occurs when $\beta<0.5$. The most unstable wavenumber is $k=\sqrt{2} / 2$, which is the retained wavenumber in the calculations presented here. The model has been integrated for 1100 non-dimensional time units for various parameter configurations. The initial conditions had small amplitudes in all the retained wavenumbers. Note that a time unit is $\lambda / U$; if we choose realistic values for the internal radius of deformation $\left(\lambda \sim 10^{6} \mathrm{~m}\right)$ and the mean vertical shear $(U \sim 10 \mathrm{~m} / \mathrm{s})$ we estimate a time unit to be about 1 day.

In this report, results from the modal variables model are presented. Figure (1) shows the experiments that were performed for various choices of $\beta$ and $\kappa$.

Figure (2) presents a table of plots of the time series of the total energy in the model for the days of integration. Note that equilibration is reached around the 600th time unit. Generally, the equilibration energy level is higher for high values of supercriticality, as expected. It appears that lower values of friction do not signify higher PV flux, a point that we return later.

Figure (3) presents a table of plots of the time series of the PV flux in the model for the integration period. For high values of supercriticality and low friction, the P.V flux seems to be fluctuating greatly. Friction seems to decrease the PV flux variance, but not affect its amplitude greatly.

In Figure (4) the energy spectra are displayed for a two experiments with varying $\beta$ and $\kappa$. If the scale of the domain was the largest scale of the flow, one would expect the wave energy spectra to strongly peak at $l=0$. Clearly, this is not the case for these particular choices of parameters. Note that the most energetic scale of the zonal mean component is not at the radius of deformation (discrete wavenumber $n=10$, or $l_{n}=1$ ) but at smaller scales around $n=5\left(l_{n}=0.5\right)$.

The Hovmuller diagrams for the same parameters choices are displayed in (5) computed at longitude $x=0$. The turbulent nature of the flow is clearly evident. Also evident are zonal jets that persist for long periods. The less supercritical experiment has smaller streamfunction amplitude although the friction is half of the more supercritical one.

Figure (6) shows the spectra of PV flux for four experiments with varying $\beta$ while the friction held at $\kappa=0.2$. Similarly, Figure (7) shows the spectra of PV flux for three experiments with varying $\kappa$ while $\beta$ is held at 0.15 . The PV flux spectra vary significantly for different values of supercriticality, while they do not appear to fluctuate greatly for varying friction. 


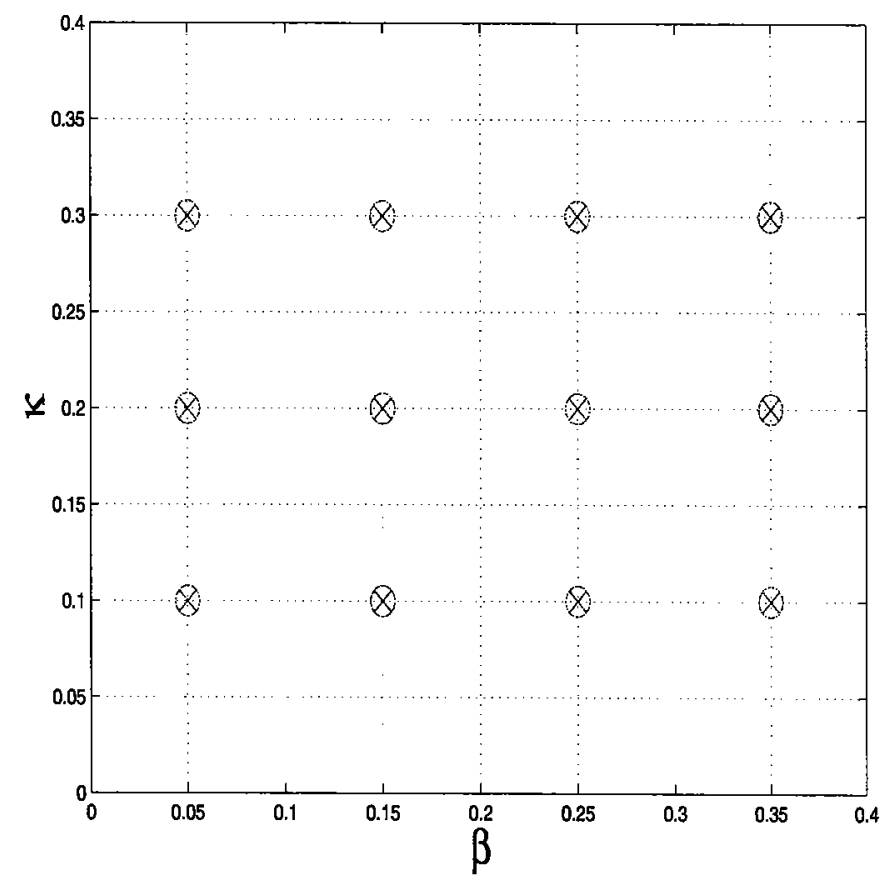

Figure 1: Performed integrations for varying $\beta$ and $\kappa$. The retained zonal wavenumber is $k=\sqrt{2} / 2$, the length of the domain is given by $L=10$ and the hyper-diffusion coefficient is set to $\nu=10^{-3}$

\subsection{Reduced Model}

[2] argued that the large-scale motion of the two layers system is dominated by the barotropic streamfunction $\psi$, while the baroclinic streamfunction $\tau$ acts as a passive tracer, and cascades downscale towards smaller scales. The same line of argument has been pursued in $[7,8]$ to provide scaling arguments for the PV flux. The baroclinic mode is assumed to be mixed downgradient by the turbulent diffusion provided by the barotropic flow.

To test this hypothesis we drop the terms in curly brackets from equation (8), (correspondingly from equations (10) and (13)) and solve the reduced system of equations.

The reduced system needs a rather drastic modification in the value of eddy viscosity that is applied to the baroclinic and barotropic wave components. The eddy viscosity applied to the zonal mean components is the same as in the full model.

This is necessary to prevent an "ultraviolet catastrophe", where the smaller meridional scales are more unstable than the larger ones. The linear growth rate of the full system along with linear growth rates of the reduced system modified by an eddy viscosity term of varying order are displayed in Figure 8 . We choose to use a second order diffusion operator with a value of 0.280 for $\nu$. This type of eddy viscosity appears to provide the best fit of the full model's linear growth rate.

The time series of the total kinetic energy, PV flux, and the kinetic energy of the zonal and wave components for each layer for the full and reduced models are shown in Figure 9. It seems that the reduced model is not able to quantitatively capture the essential physics of the full model. Its PV flux is much lower (almost half) than the full model. The total 

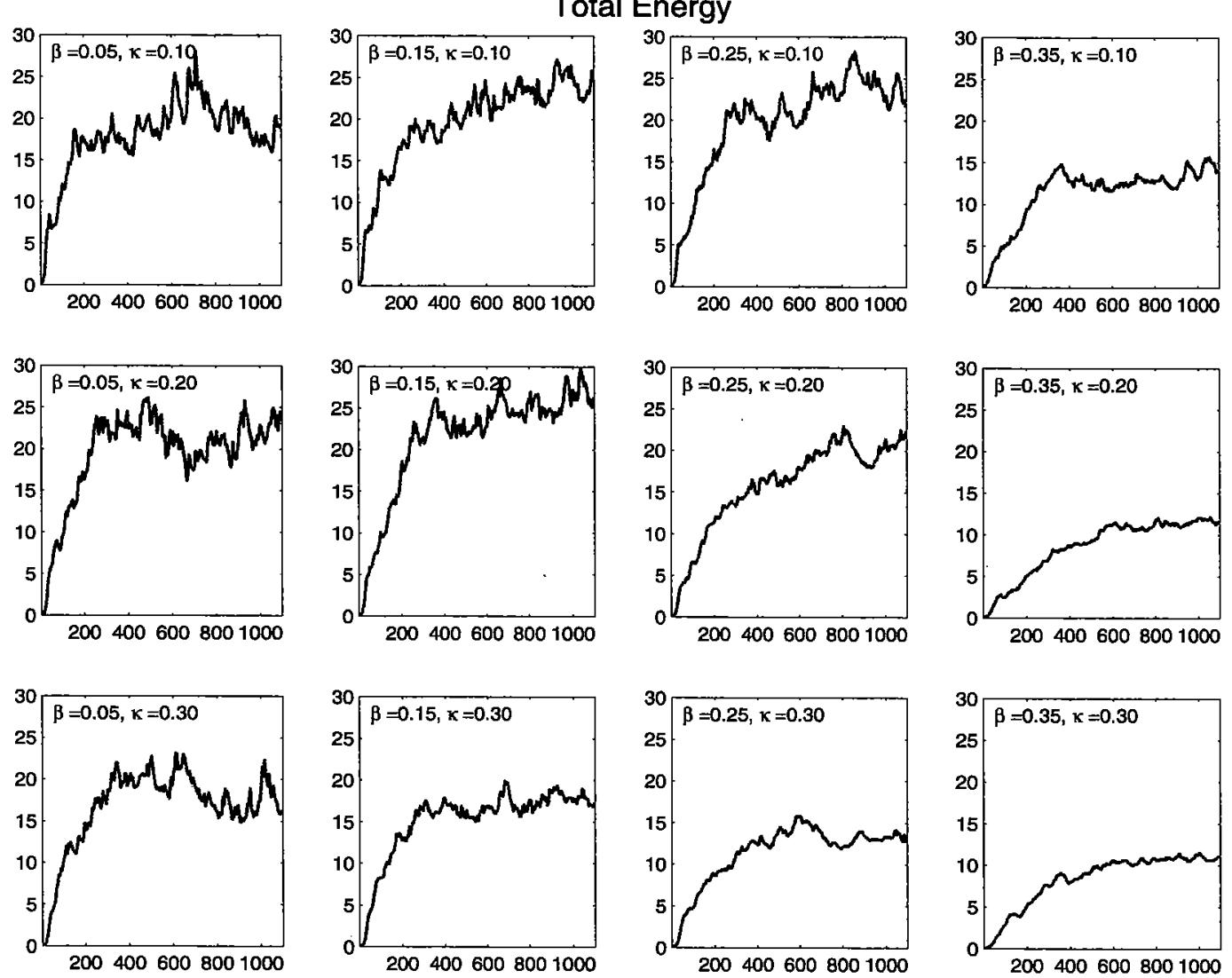

Figure 2: Table of time series of total energy for the integration period.

energy never increases to the level of the full model, mainly because the upper layer kinetic energy remains very low. This seem to be a side effect of the eddy viscosity that we use in our attempt to parameterize the neglected terms of the full model. Clearly, more effort needs to be put in quantitatively using a reduced model like ours.

\section{Sensitivity Experiments}

Several experiments were performed spanning a moderate area of the parameter space (see Figure 1). The objective of the multiple runs is to gain an overview of the behavior of the PV flux for different choice of parameters.

Figures 10 and 11 display the computed time averaged PV flux for various choices of $\beta$ and $\kappa$.

An interesting feature of the PV flux dependence on friction is shown in 11 . There is a local maximum in PV flux around $\kappa=0.20,0.15$. The decreasing fluxes for increasing $\kappa$ may be readily understood as the direct effects that friction has on the energy that is transported in the system. However, the decrease in PV flux for decreasing $\kappa$ may be understood in different terms. The equilibrated system experiences barotropic eddies that the size depends 

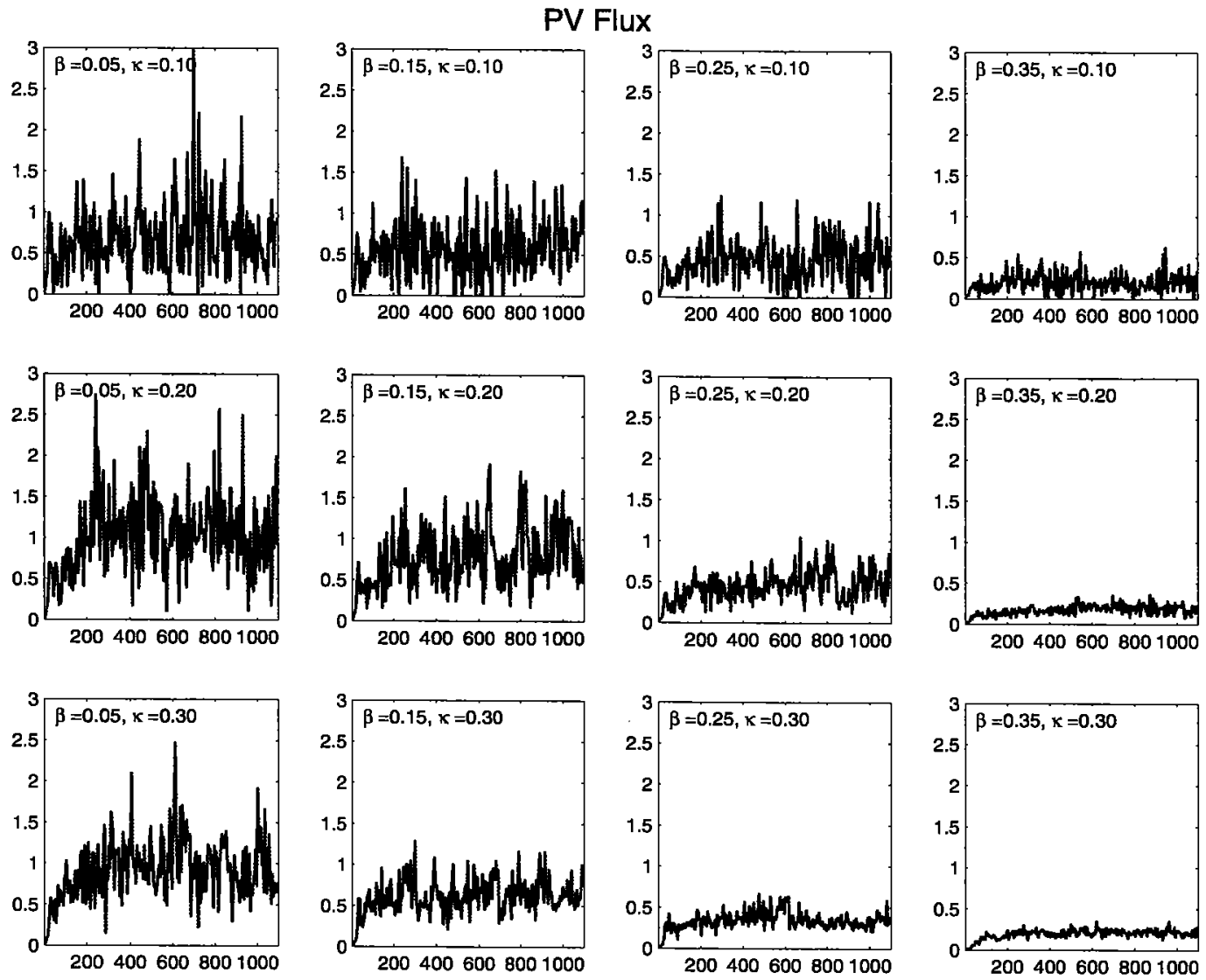

Figure 3: Table of time series of PV flux for the integration period.

on their frictional dissipation. [2] has hypothesized that these barotropic eddies strain the unstable baroclinic eddies so that their meridional wavenumber becomes very large and their linear growth rate decreases. Weaker friction will permit the barotropic energy to cascade to large barotropic eddies (mainly zonal) that are less efficient in providing PV fluxes, while they will strain all the smaller baroclinic eddies. Thus, the local maximum in PV flux would correspond to a value of the frictional parameter $\kappa$ for which the straining of the unstable baroclinic eddies is not as efficient and the transport accomplished by the barotropic eddies is not small. It appears that for higher $\beta$ (i.e. lower supercriticality) the local maximum is shifted towards lower $\kappa$ 's indicating that the straining mechanism hypothesized above is performed by the large barotropic eddies left by friction in the system, at sizes larger than their counterparts in a more supercritical system.

As expected, Figure 10 shows that the PV flux generally increases with decreasing nondimensional $\beta$ (i.e. increasing supercriticality). The same type of result has been presented in [4] (their Figure 4d) and has been added here for comparison. Some interesting behavior is displayed at intermediate values of $\beta$ when $\kappa$ is 0.1 and 0.3 . This may be understood in terms of the behavior that the eddies have been hypothesized to acquire in the above paragraph. 


\section{Conclusions and Future Work}

In this work we use a quasi-geostrophic, two-layer model, severely truncated in the zonal direction, to understand the eddy PV fluxes. To ascertain homogeneity, a doubly periodic boundary condition was used. To enable an inertial range of enstrophy cascade, a large resolution is kept in the meridional direction. The system then equilibrates by reducing the northward heat flux $\left\langle\psi_{x} \tau\right\rangle$, i.e. the correlation of the northward velocity with temperature, and by non-linear transfer of energy from unstable baroclinic waves to stable ones (or less unstable) through triad interactions and consequent frictional dissipation ([2]).

It was also attempted to reduce the model to one that has guided many qualitative arguments (e.g. [7], [2] ) concerning the parameterization of eddy PV fluxes. Unfortunately, quantitative use of this kind of reduced system does not appear to be fruitful. To avoid an "ultraviolet catastrophe" in the reduced model, where the waves with larger wavenumber grow the fastest, the eddy viscosity assumes the role of the neglected terms to stabilize the large wavenumbers. In this case, the gross statistics of the reduced model do not resemble the ones of the full model. It may be possible, that another way of preventing the smaller waves to grow without bound exists, in which case the reduced model may be revisited.

The eddy PV flux in our system appears to depend only marginally on the frictional parameter $\kappa$. An interesting behavior, where there is a local maximum around $\kappa=0.2$ was noted and qualitatively discussed. The dependence on the $\beta$ parameter (or inverse supercriticality) seems to be linear. The linear growth rate of the most unstable wave, when friction is taken into account, incorporates both $\beta$ and $\kappa$, which are the only free parameters in our system. When PV flux is plotted against it, an approximate quadratic dependence appears, although the error of fit seems to be large.

More intense exploration of the parameter space seems appropriate to further establish any relationship of the eddy PV fluxes to the free parameters of the problem. A theory may be developed for PV flux parameterization in the framework of the truncated model once these relationships are more thoroughly studied. The reduced model could be possibly used either qualitatively, or quantitatively.

\section{Acknowledgments}

The constant guidance of Rick Salmon and Isaac Held, and the invaluable discussions we had throughout the summer are greatly appreciated. Thanks are due to the GFD program staff and all the visitors that constantly kept an intellectually stimulating atmosphere around Walsh Cottage. Last, but certainly not least, I'd like to thank my fellow Fellows for an unforgettable summer. I also gladly acknowledge the significant contribution of the beaches of Woods Hole, that were a true inspiration for hard work :-)

\section{References}

[1] R. Salmon, "Two-layer quasi-geostrophic turbulence in a simple case," Geophys. Astrophys. Fluid Dyn. 10, 25 (1978). 
[2] R. Salmon, "Baroclinic instability and geostrophic turbulence," Geophys. Astrophys. Fluid Dyn. 15, 167 (1980).

[3] D. B. Haidvogel and I. M. Held, "Homogeneous quasi-geostrophic turbulence driven by a uniform temperature gradient," J. Atmos. Sci. 37, 2644 (1980).

[4] R. L. Panetta and I. M. Held, "Baroclinic eddy fluxes in a one-dimensional model of quasi-geostrophic turbulence," J. Atmos. Sci. 45, 3354 (1987).

[5] J. R. Holton, "A semi-spectral numerical model of wave-mean flow interactions in the stratosphere: Application to sudeen stratospheric warmings," J. Atmos. Sci. 33, 1639 (1971).

[6] I. M. Held and M. J. Suarez, "A two-level primitive equation atmospheric model designed for climate sensitivity experiments," J. Atmos. Sci. 35, 206 (1980).

[7] V. D. Larichev and I. M. Held, "Eddy amplitudes and fluxes in a homogeneous model of fully developed baroclinic instability," J. Phys. Ocean. 25, 2285 (1995).

[8] I. M. Held and V. D. Larichev, "A scaling theory fo horizontally homogeneous, baroclinically unstable flow on a beta plane," J. Atmos. Sci. 53, 946 (1996). 

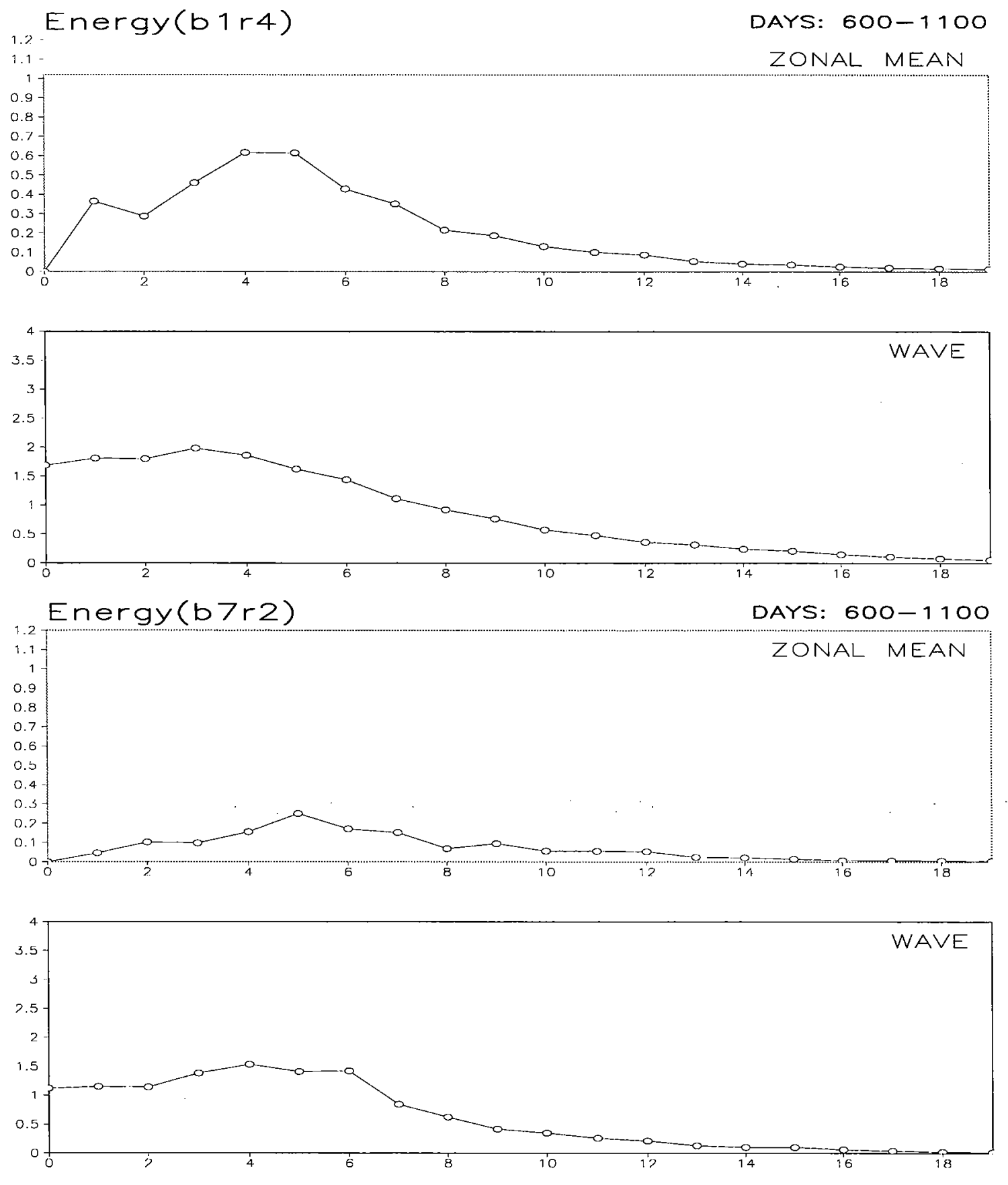

Figure 4: Energy spectra for two runs: top: $\beta=0.05$, and $\kappa=0.2$, bottom: $\beta=0.35$, and $\kappa=0.1$ 

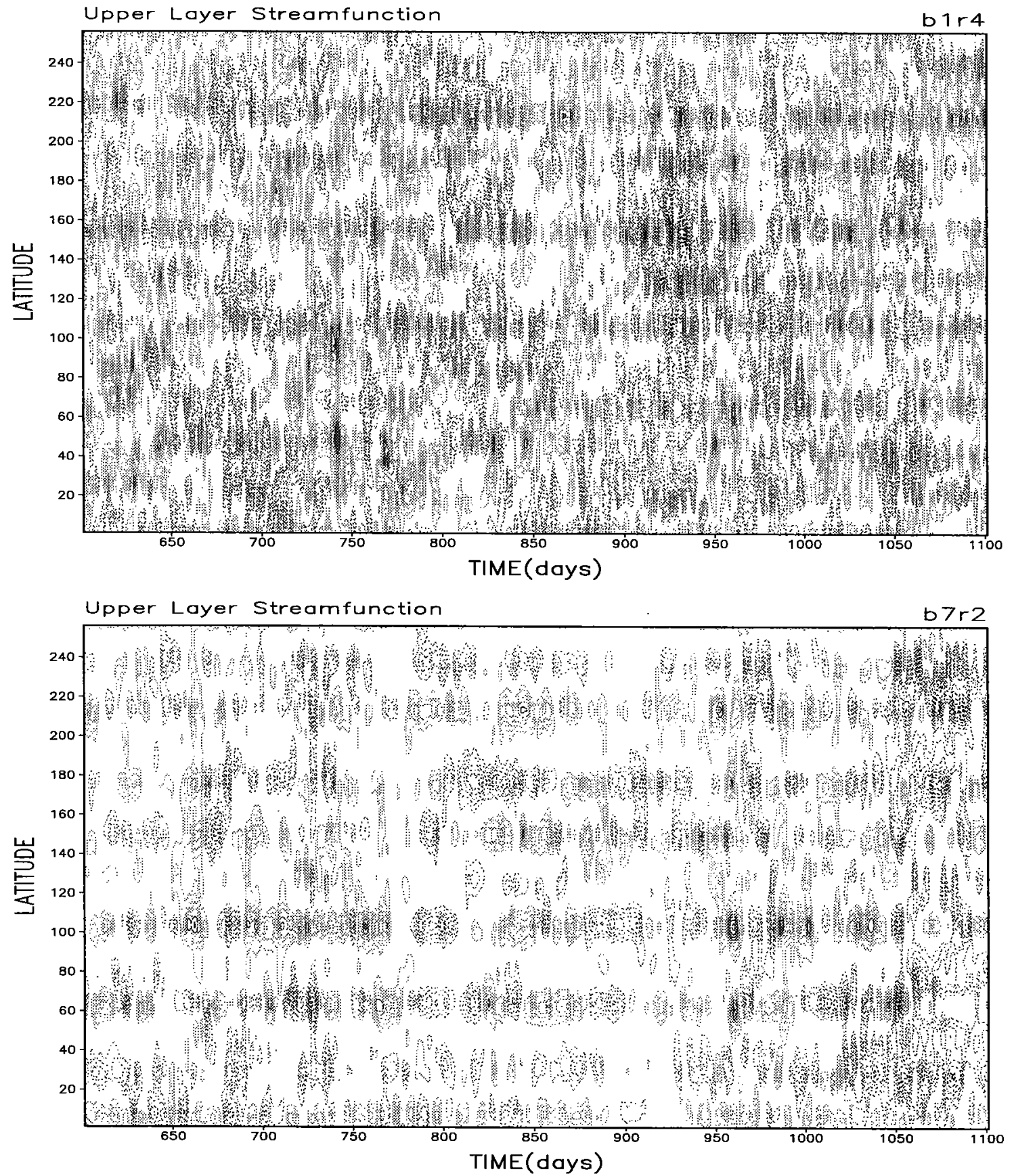

Figure 5: Hovmuller diagrams for two runs: top: $\beta=0.05$, and $\kappa=0.2$, bottom: $\beta=0.35$, and $\kappa=0.1$ 


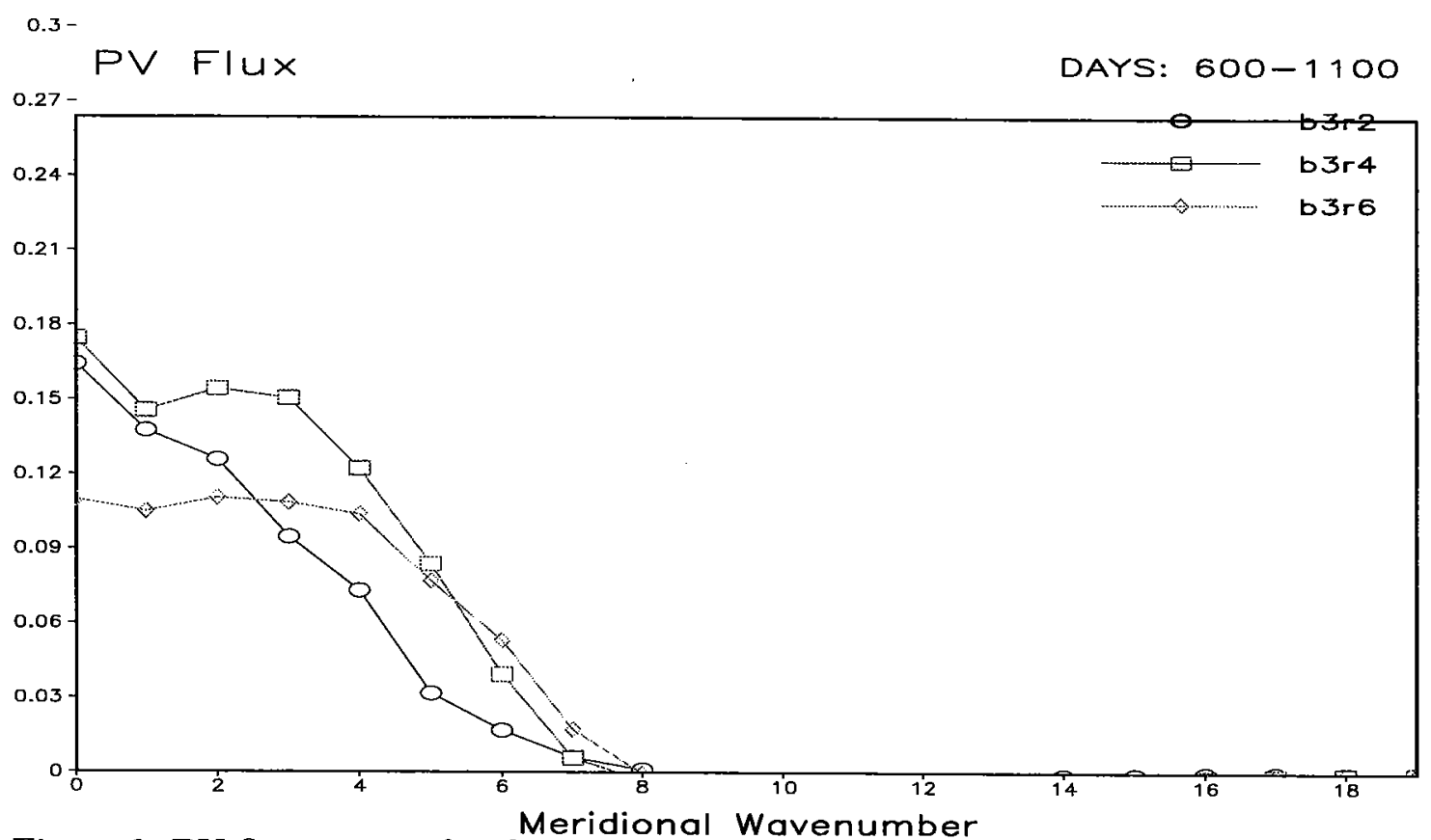

Figure 6: PV flux spectra for three experiments with varying $\kappa$ while $\beta$ is held at 0.15 .

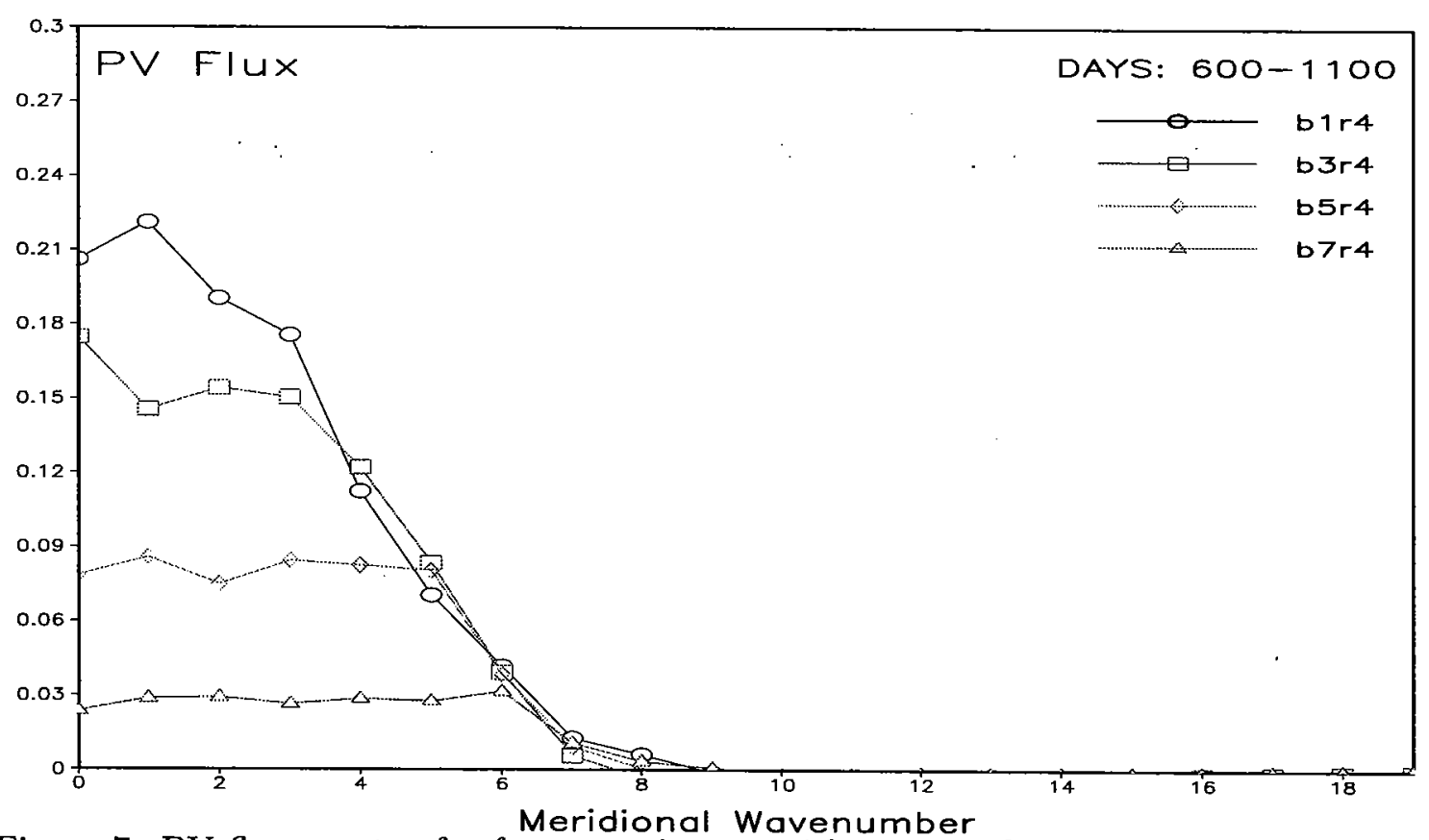

Figure 7: PV flux spectra for four experiments with varying $\beta$ while the friction held at $\kappa=0.2$. 


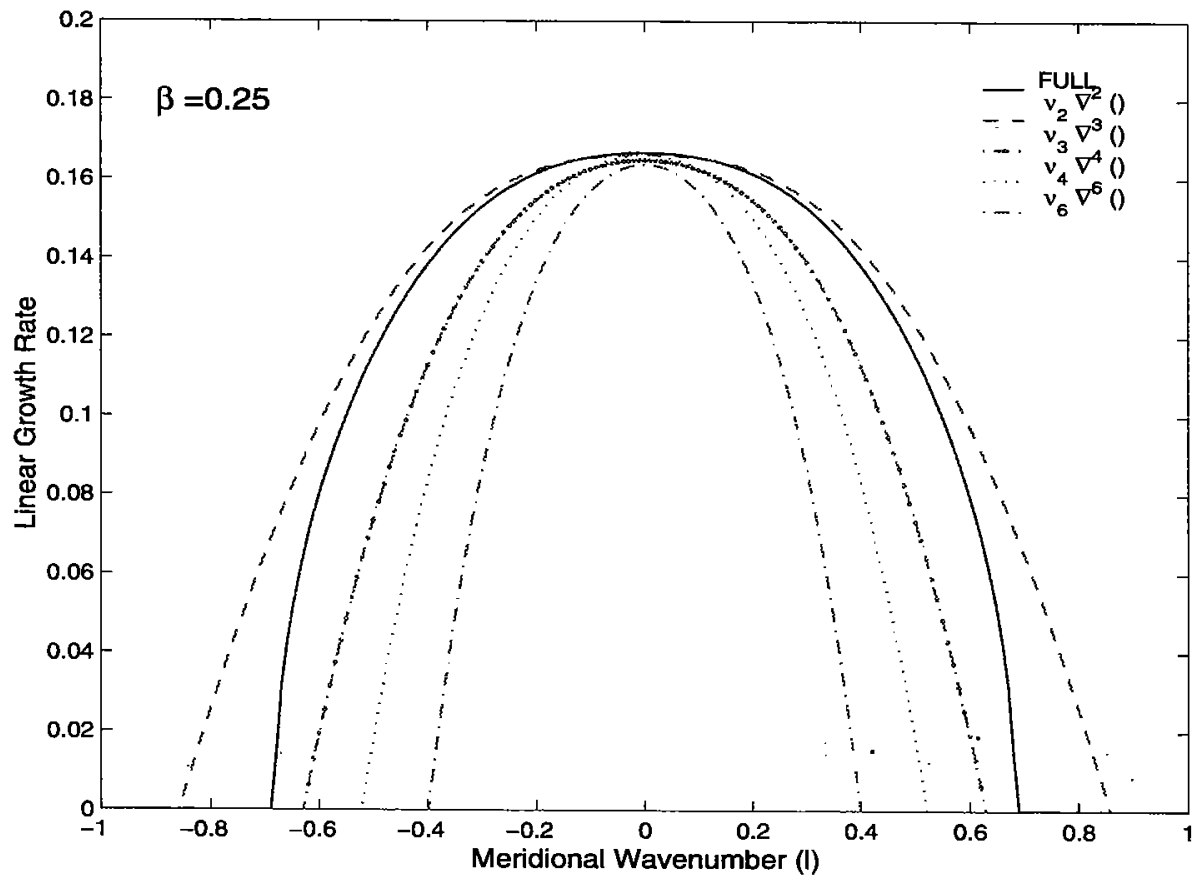

Figure 8: Linear growth rate as a function of meridional wavenumber $(l)$ for the full model, and for the reduced model with modified eddy viscosity of degrees $2,3,4$ and 6 . $\beta$ is set to 0.25 

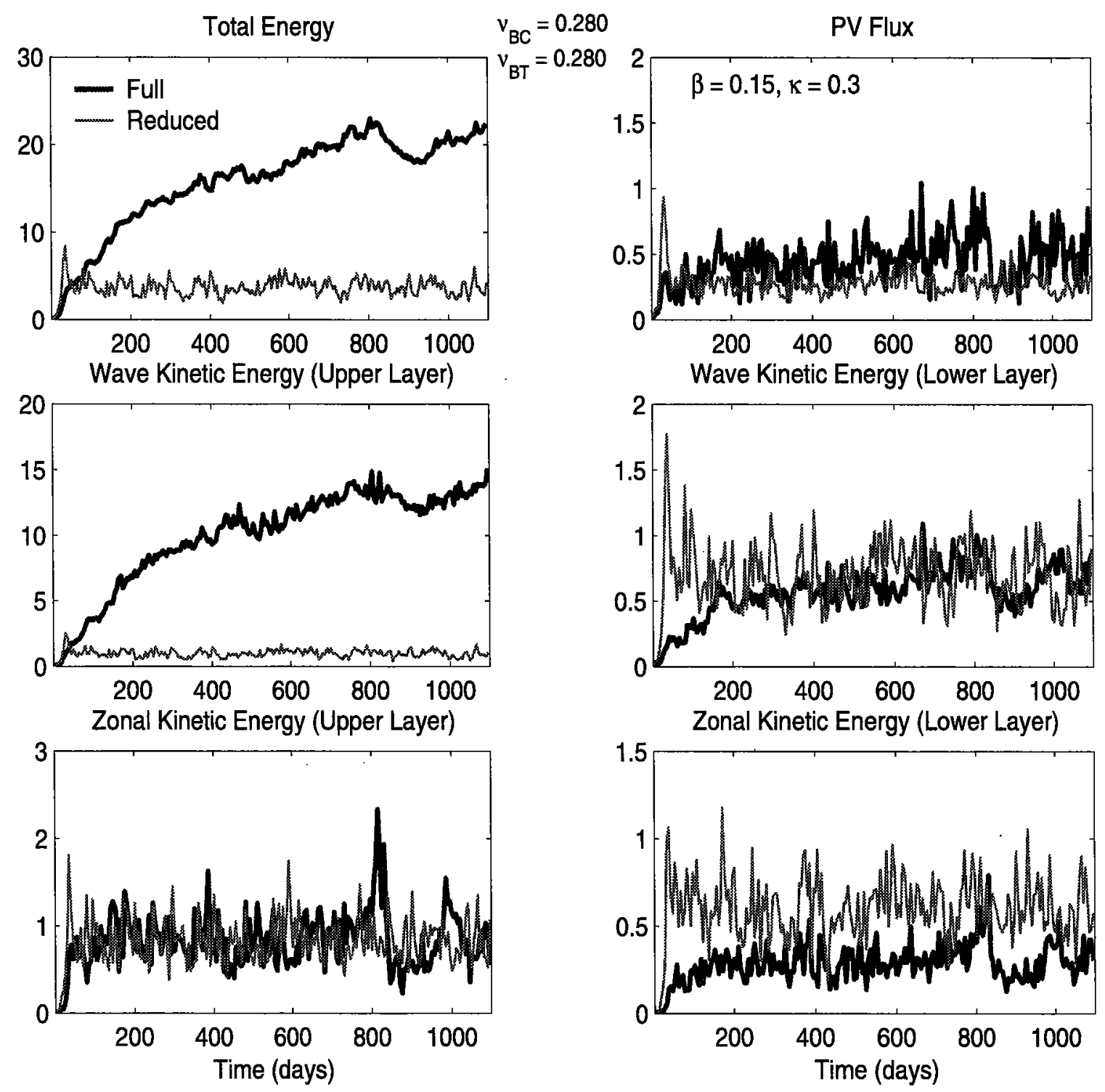

Figure 9: Total kinetic energy, PV flux, and kinetic energy of the zonal and wave components for the two layers for the full and the reduced models. 


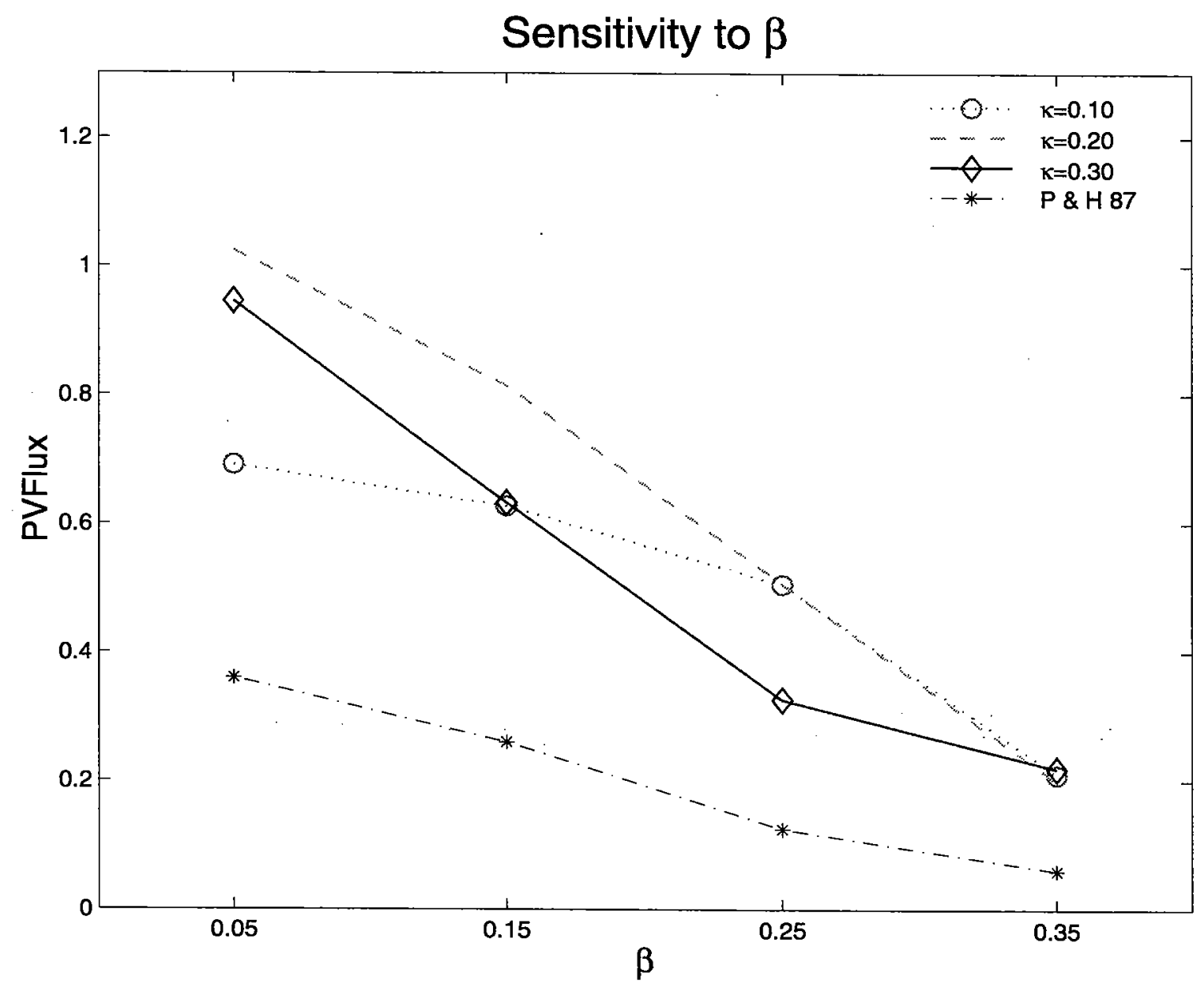

Figure 10: Time averaged behavior of equilibrated PV flux for varying $\beta$ and $\kappa$ instances. Results from [4] that include thermal damping are also included for comparison. 


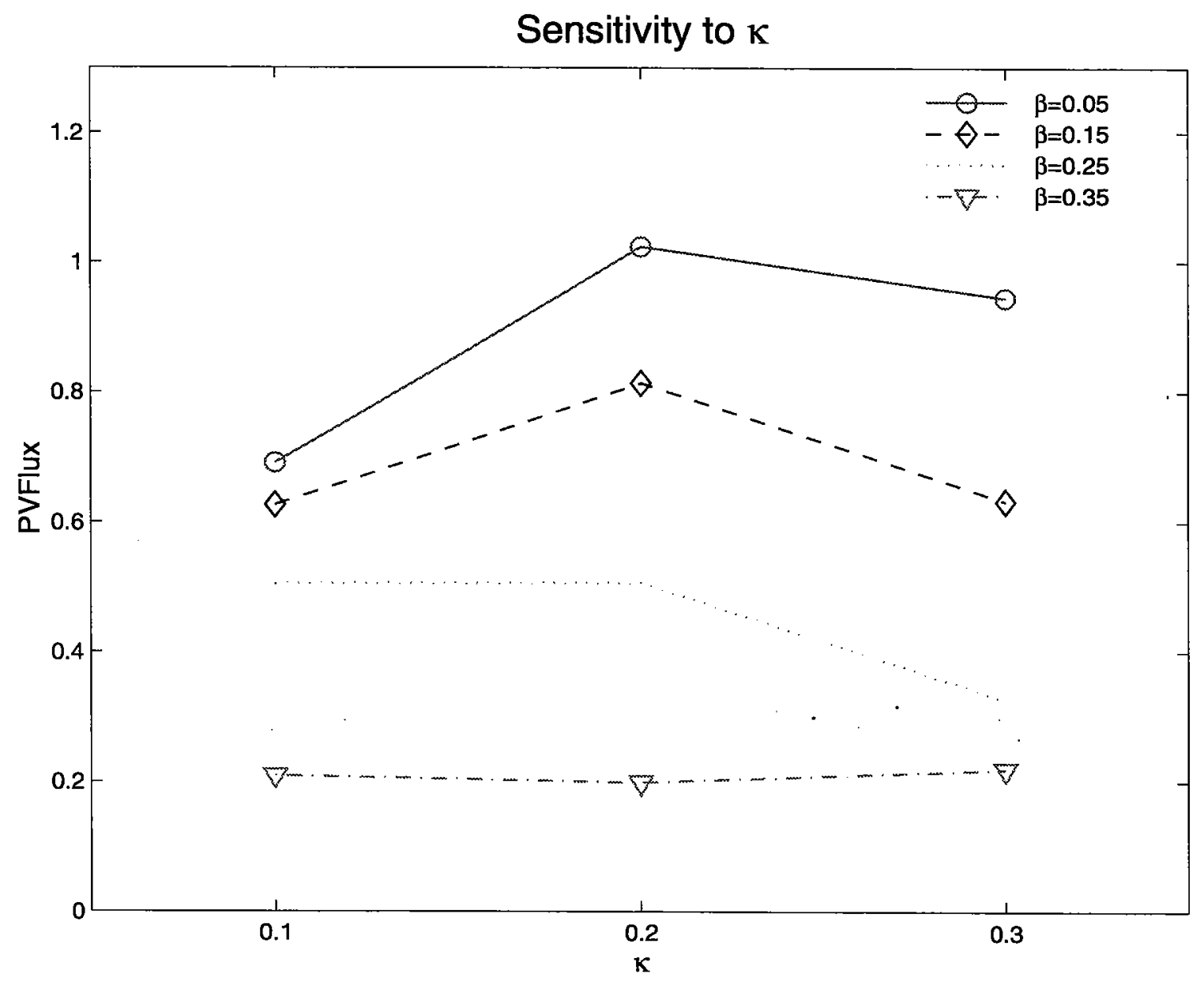

Figure 11: Time averaged behavior of equilibrated PV flux for varying $\beta$ and three $\kappa$ instances. 


\section{Sensitivity to Linear Growth Rate}

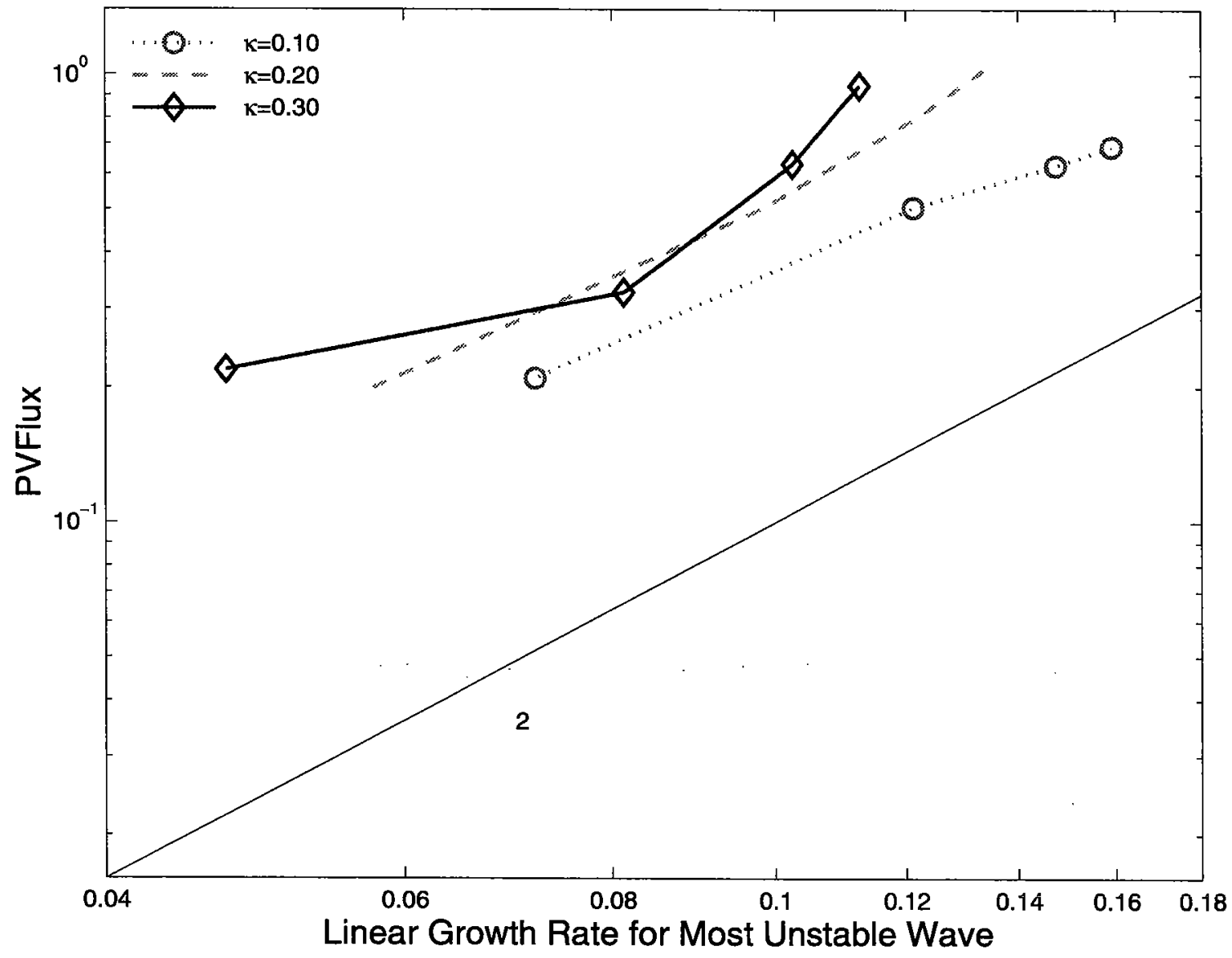

Figure 12: Time averaged behavior of equilibrated PV flux for varying and $\kappa$ and $\beta$ instances. 


\title{
Superrotation in an Axisymmetric Shallow Water Model of the Upper Troposphere
}

\author{
Karen M. Shell
}

\section{Introduction}

Superrotation is defined by zonal mean zonal winds that have a greater angular momentum than the surface of the earth at the equator. In order for the circulation to be inertially stable, the angular momentum of the zonal winds must decrease poleward. Thus, if the winds at the equator are not superrotating, the winds are nowhere superrotating.

On the earth, the equatorial tropospheric winds are slightly easterly, so they have less angular momentum than the earth's surface at the equator. Thus, the earth's troposphere is not superrotating. However, superrotation occurs during the westerly phase of the QuasiBiennial Oscillation (QBO) in the stratosphere, as well as on other planets, such as Jupiter and Saturn, and on our sun. These cases raise the question of whether the earth's troposphere could be superrotating under earth-like conditions.

Superrotation has appeared in simple models of the earth's atmosphere. Suarez and Duffy [1] obtained superrotating states in a two-layer model when they applied a zonally asymmetric tropical heating. For certain strengths of the forcing, they found multiple equilibria. Once superrotation was established in the model, the system would remain superrotating if they removed the asymmetric heating. Saravanan [2] used a two-layer model as well but applied a torque rather than asymmetric heating to produce superrotation. Recently, Huang et al [3] found slight superrotation in a coupled GCM climate change simulation with tripled $\mathrm{CO}_{2}$. This experiment suggests the possibility that superrotation could develop due to anthropogenic climate change, though much more work is needed to determine if this is a realistic possibility. Held summarizes the research on superrotation in earth-like atmospheric models [4].

Suarez and Duffy and Saravanan attributed the bifurcations they obtained to a feedback between the forcing (whether generated by asymmetric heating or an applied torque) and the eddies. The forcing accelerates equatorial winds, and the eddies tend to decelerate equatorial winds. However, the effectiveness of the eddies decreases as the wind speed increases. Thus, increasing the forcing leads to stronger equatorial winds and thus less deceleration by the eddies, resulting in a positive feedback.

We looked for a similar bifurcation in an axisymmetric shallow water model of the upper troposphere. The model includes a torque that is directly applied to the equatorial region. In addition, the transport of mass (and thus momentum) from a non-moving lower layer can decelerate the flow around the equator. This deceleration depends on the wind speed 
at the equator, which depends on the strength of the forcing. We are interested in whether this feedback allows multiple equilibria for some ranges of the forcing. Since $u$ is always superrotating with the applied force, we looked for multiple steady superrotating states, one weakly superrotating and one strongly superrotating, for the same set of parameters.

We attempted to add a parameterization of breaking Rossby waves to the model in order to see how waves affect the circulation. However, we were not successful because we could not get final states independent of the grid spacing.

Section 2 describes the shallow water model we used. In Section 3, we examine a simple analytical approximation to the full model to better understand the system. Section 4 describes the results of the numerical simulations. Our attempt to add breaking Rossby waves is discussed in Section 5. Finally, we present conclusions from this project in Section 6 .

\section{The Model}

We modeled the troposphere using an axisymmetric (no variation in the longitudinal direction) one-and-a-half layer isentropic model on a sphere. The lower layer does not move, but it can exchange mass with the upper layer, thus affecting the height and zonal velocity of the upper layer. The upper portion of the troposphere is modeled using the shallow water equations for a spherical isentropic layer. The model determines the zonal velocity, $u$, meridional velocity, $v$, and height of the upper layer, $h$, as a function of time, $t$, and latitude, $\phi$ :

$$
\begin{gathered}
\frac{\partial u}{\partial t}+\frac{v}{a} \frac{\partial u}{\partial \phi}-2 \Omega v \sin \phi-\frac{u v \tan \phi}{a}=F+R-k u \\
\frac{\partial v}{\partial t}+\frac{v}{a} \frac{\partial v}{\partial \phi}+2 \Omega u \sin \phi+\frac{u^{2} \tan \phi}{a}=-\frac{g^{*}}{a} \frac{\partial h}{\partial \phi}-k v \\
\frac{\partial h}{\partial t}+\frac{1}{a \cos \phi} \frac{\partial h v \cos \phi}{\partial \phi}=-\frac{h-h_{e q}}{\tau}
\end{gathered}
$$

where $F$ is an applied forcing. The system is relaxed to a radiative equilibrium height, $h_{e q}(\phi)$, and

$$
R= \begin{cases}\frac{h-h_{e q}}{\tau} \frac{u}{h} & \left(h-h_{e q}<0\right) \\ 0 & \left(h-h_{e q}>0\right)\end{cases}
$$

is the deceleration of the zonal momentum due to mass moving from the lower layer to the upper layer as part of this height relaxation. $\Omega$ is the rotation rate, $a$ is the radius of the earth, $k$ is the frictional parameter, $g^{*}$ is the reduced gravity, and $\tau$ is the relaxation time. All values are in MKS units.

The forcing $F$ for the system is an applied torque centered around the equator and constant in time. Two types of forcing are used. (Figure 1 illustrates some of the forcing distributions.)

1. The first torque represents a flux of momentum from the lower layer:

$$
F_{1}=F_{0} \cos ^{n} \phi
$$

where $F_{0}$ is the forcing at the equator. 


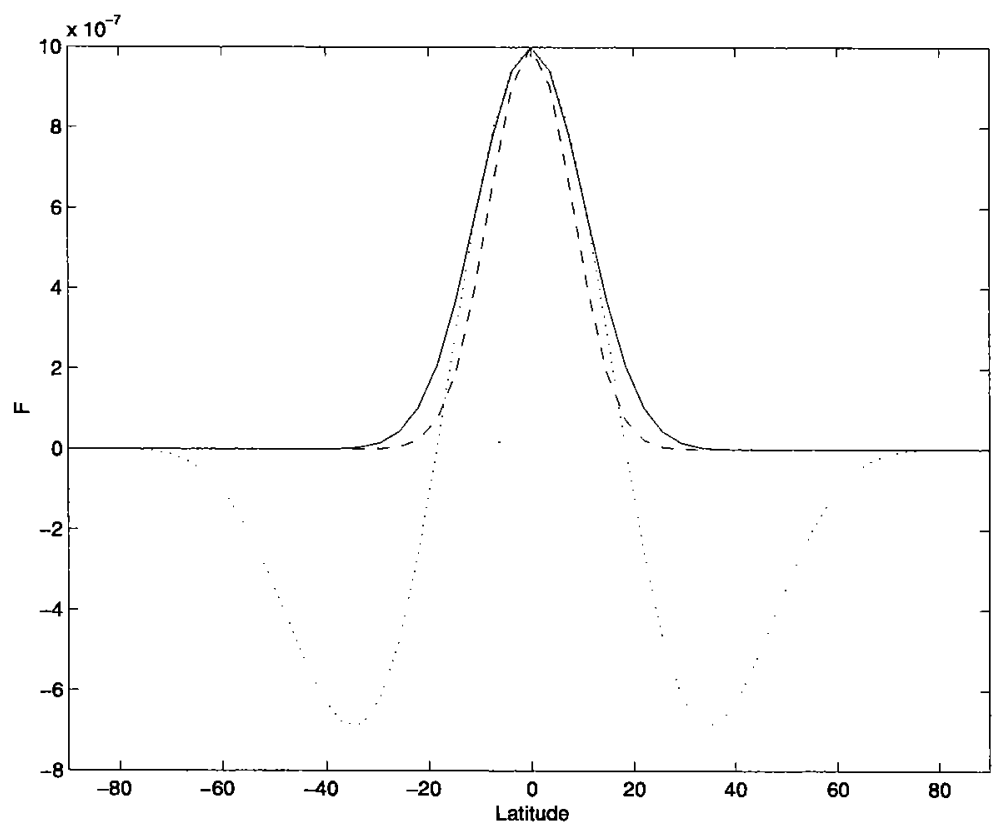

Figure 1: Example forcing distributions for $F_{0}=10^{-6}$. The solid line corresponds to the default forcing distribution $\left(F_{1}, n=30\right)$. The dashed line corresponds to a narrower forcing $\left(F_{1}, n=50\right)$. The dotted line corresponds to an angular momentum conserving forcing $\left(F_{2}\right.$, $n=9$ ). For a given value of $F_{0}$, the forcing distributions all have the same value at the equator.

2. The second torque represents a redistribution of angular momentum within the layer:

$$
F_{2}=F_{0} \frac{h_{0}}{h}\left(-n \cos ^{n-3} \phi+(n+1) \cos ^{n-1} \phi\right),
$$

where $h_{0}$ is the height of the layer at the equator. This forcing accelerates winds in the tropics and decelerates them in the midlatitudes, corresponding to waves excited in the tropics and breaking in the midlatitudes.

The height of the upper layer is relaxed back to the "radiative equilibrium" height, $h_{e q}$, which simulates the effects of radiation on the system. When radiation warms a portion of air in the lower layer, its potential temperature increases, and it moves to the upper layer, increasing the height of the upper layer. When the upper layer cools, some air moves down into the lower layer, decreasing the height of the upper layer. The radiative equilibrium is assumed to be in geostrophic balance with the "radiative equilibrium" wind:

$$
u_{e q}=u_{0 e q} \cos \phi
$$

where $u_{0 e q}$ is the radiative equilibrium wind at the equator. By integrating the geostrophic terms in equation (2):

$$
2 \Omega \sin \phi u_{e q}=-\frac{g^{*}}{a} \frac{\partial h_{e q}}{\partial \phi}
$$


we obtain the equation for $h_{e q}$ :

$$
h_{e q}=h_{0 e q}-\frac{a \Omega u_{0 e q}}{g^{*}} \sin ^{2} \phi,
$$

where $h_{0 e q}$ is the radiative equilibrium height at the equator.

The relaxation back to the equilibrium height also affects the zonal momentum budget through term $R$ in equation (1). Air that is brought up from the lower layer carries with it the momentum from the lower layer. Since the momentum of the lower layer is less that that of the upper layer, this process decreases the zonal momentum in the upper layer. However, air that moves from the upper layer down to the lower layer carries with it the momentum of the upper layer and thus does not affect the momentum in the upper layer. Presumably surface friction returns the velocity in the lower layer to zero. Note that the relaxation mass flux also should affect the meridional velocity. However, we have omitted this effect from equation (2) because $v$ is very close to geostrophic balance.

To derive the effect of height relaxation on the zonal velocity, start by changing $h$ based on the amount of mass added to the layer:

$$
\frac{\partial h}{\partial t}=-\frac{h-h_{e q}}{\tau}
$$

The total angular momentum in the upper layer, $h M$, changes by the amount of mass added times the absolute angular momentum of the moving mass, $M_{r}$ :

$$
\frac{\partial(h M)}{\partial t}=-\frac{h-h_{e q}}{\tau} M_{r}
$$

Using the chain rule and substituting in equation (6) yields

$$
\frac{\partial M}{\partial t}=-\frac{\left(h-h_{e q}\right)}{\tau} \frac{\left(M_{r}-M\right)}{h} .
$$

Since

$$
M=(\Omega a \cos \phi+u) a \cos \phi,
$$

we obtain an expression for the change to the zonal velocity:

$$
\frac{\partial u}{\partial t}=-\frac{\left(h-h_{e q}\right)}{\tau} \frac{\left(u_{r}-u\right)}{h} .
$$

When $h-h_{e q}$ is positive, $u_{r}=u$ because the mass that moves has the same velocity as the layer. When, $h-h_{e q}$ is negative, $u_{r}$ is the zonal velocity of the lower layer. Since the lower layer is not moving, we obtain equation (4). This term is the key term in our model, because it provides the positive feedback to the forcing. At equator, the forcing must balance the height relaxation term and friction. Since friction increases as the forcing (and thus the wind speed) increases, the height relaxation term is the required term for a bifurcation in this system. 


\subsection{Numerical Scheme}

The model is solved numerically using a centered in space, leapfrog in time scheme with a Robert (asselin) filter to prevent the even and odd time time steps from drifting apart. The filter is applied to $u, v$, and $h$ after the value at time $t+1$ is calculated and has the following form:

$$
X_{\text {new }}^{t}=(1-2 \alpha) X_{\text {old }}^{t}+\alpha\left(X^{t-1}+X^{t+1}\right)
$$

The value of $\alpha$ does not affect the results, since we are interested in finding steady states. Note, however, that the choice of $\alpha$ might affect periodic solutions.

The value of the time step, $\Delta t$, does not affect the solution; however, if it is too high, the solution becomes unstable. Generally, I used values between 250 and 500 seconds.

The grid is staggered, with $u$ and $h$ gridpoints halfway between the $v$ grid points. The staggered grid point method allows for easier calculation of gravity waves. The poles correspond to $v$ grid points, and $v$ is set to 0 here. The equator corresponds to a $u$ and $h$ grid point.

The advective and Coriolis terms of Equation 1 are actually calculated in flux form as

$$
\frac{\partial u}{\partial t}=\frac{1}{a \cos \phi} \frac{\partial M}{\partial t}
$$

where $M$ is the total angular momentum and is calculated so that

$$
\frac{\partial(M h)}{\partial t}=h \frac{\partial M}{\partial t}+M \frac{\partial h}{\partial t}
$$

\subsection{Model without forcing}

Figure 2 shows the steady state of the model with no forcing. For this run, we used values of $a=6.37 \times 10^{6} \mathrm{~m}, \Omega=7.292 \times 10^{-5} \mathrm{rad} / \mathrm{s}, g^{*}=9.81 \times 0.1 \mathrm{~ms}^{-2}, \tau=900000 \mathrm{~s}, k=5 \times 10^{-9}$ $\mathrm{s}^{-1}, h_{0 e q}=56632 \mathrm{~m}, u_{0 e q}=100 \mathrm{~m} / \mathrm{s}$, and $\alpha=0.5$. The number of $v$ grid points is 200 . Except for the grid spacing, which is normally $50 v$ grid points, these are the default values for all runs.

While the model uses similar parameters to those of the earth, it is not entirely earthlike. First, the atmosphere is not well-represented as two isentropic layers. The difference in potential temperature between the equator and the poles is about the same as the difference between the surface and the tropopause. Thus, the real atmosphere does not have an isentrope which divides the troposphere into an upper and lower layer.

The height of the layer is too large because the radiative equilibrium $u, u_{e q}$ is too large in the high latitudes. (Perhaps a different function for $u_{e q}$ should be used, but the cosine function allows us to use a simple analytical model of the more complex computer model.) Since $h$ is so large, the Hadley circulation is very weak, so the meridional velocity is smaller than it should be.

\section{Analytical Calculations}

In order to study the system more closely, we used a simple analytical model which approximates the full system of equations. The analytical model seeks to describe the state 

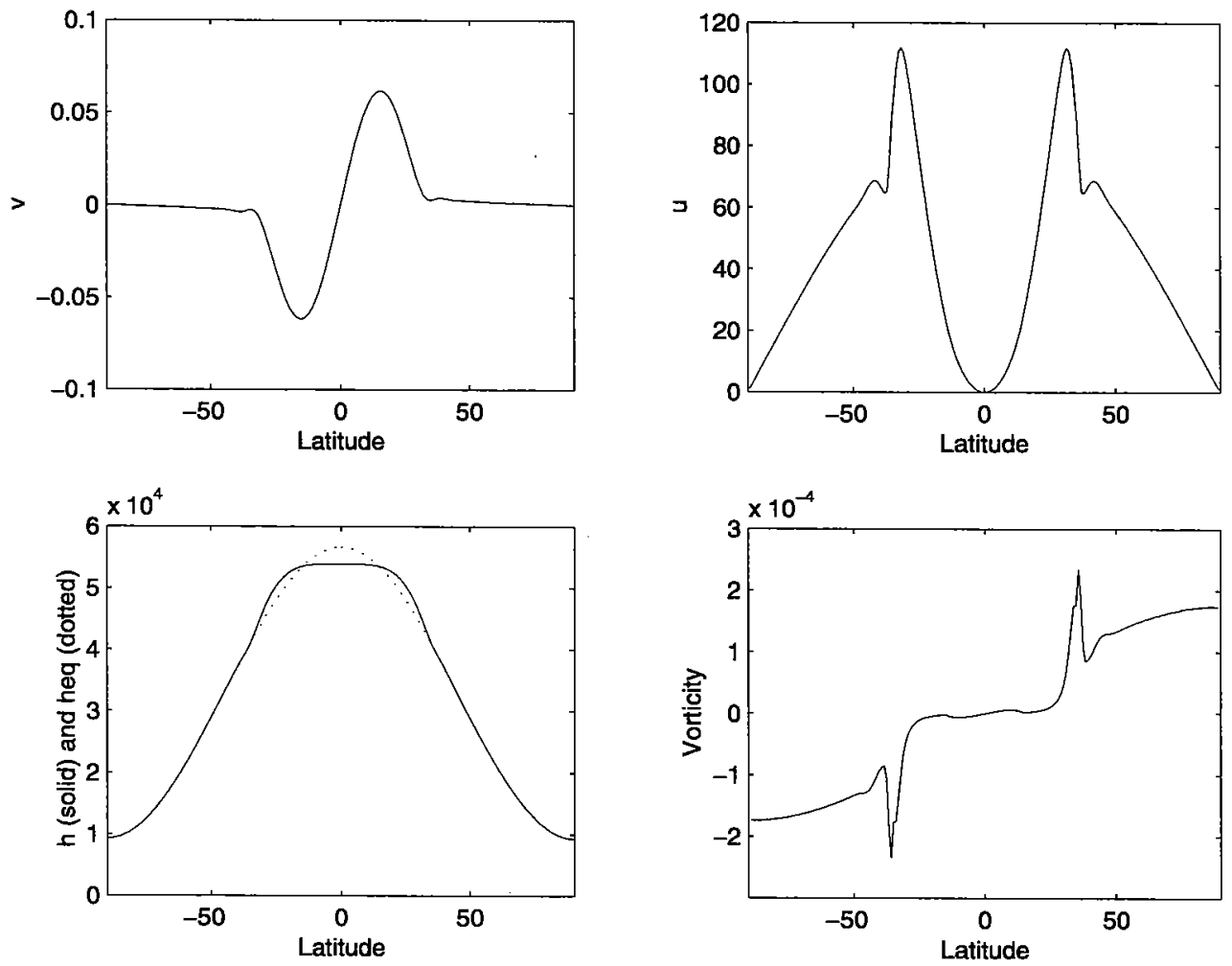

Figure 2: Steady state of the model with no forcing.

of the system using only $u$ and $h$ at the equator. By requiring that $u$ and $h$ are in steady state at the equator, we can determine which of these states is an equilibrium solution for a given set of parameters. This information gives us an idea of where to look for multiple equilibria.

The relation of the circulation to $u$ and $h$ at the equator can be explored using a simple Hadley cell model similar to the one used in Held and Hou [5]. The domain is divided into two regions. Close to the equator, the solution for $h$ conserves angular momentum, while towards the poles, the solution is just the "radiative equilibrium" solution, $h_{e q}$. By matching these two solutions, we can determine the critical latitude, $\theta_{c}$, which separates them.

Equation (5) describes the radiative equilibrium solution. To determine the angular momentum conserving solution, $h_{m}$, assume the height is in geostrophic balance with the angular momentum conserving wind:

$$
u_{m}=\frac{u_{0}+\Omega a \sin ^{2} \phi}{\cos \phi}
$$

where $u_{0}$ is the zonal velocity at the equator. Integrating the geostrophic terms in equation (2) using the small angle approximation and the fact that $u_{0}<<\Omega a$,

$$
h_{m}=h_{0}-\frac{2 a \Omega}{g^{*}}\left[u_{0} \frac{\phi^{2}}{2}+\Omega a \frac{\phi^{4}}{4}\right]
$$


where $h_{0}$ is the height of the layer at the equator.

Continuity of $h$ at $\phi_{c}$ requires

$$
h_{e q}\left(\phi_{c}\right)=h_{m}\left(\phi_{c}\right)
$$

while mass conservation requires

$$
\int_{0}^{\phi_{c}} h_{e q} \cos \phi d \phi=\int_{0}^{\phi_{c}} h_{m} \cos \phi d \phi .
$$

Solving equations (9) and (10) assuming $u_{0}<<\Omega a, h_{0 e q} \approx h_{0}$, and $\phi_{c}$ small yields equations for the critical latitude and the height at the equator in terms of $u_{0}$ :

$$
\begin{aligned}
\phi_{c}^{2} & =\frac{5}{3} \frac{u_{0 e q}-u_{0}}{\Omega a} \\
h_{0}-h_{0 e q} & =-\frac{5}{18 g^{*}}\left(u_{0 e q}-u_{0}\right)^{2} .
\end{aligned}
$$

Note that to get a real value for $\phi_{c}, u_{0}$ must be less than $u_{0 e q}$. When these computations are performed without the approximations for the parameter range we use, the results agree fairly well with this equation (Moehlis, personal communication), so we feel that the approximations are valid.

In order for $u_{0}$ and $h_{0}$ to be in steady state, the applied torque at the equator must balance the friction and the momentum exchange due to relaxation of the height to equilibrium:

$$
F=\frac{h_{0 e q}-h_{0}}{\tau} \frac{u_{0}}{h_{0}}+k u_{0} \quad\left(h_{0}<h_{0 e q}\right) .
$$

Assuming the system is not far from radiative equilibrium, we approximate the height in the denominator as a constant, $h_{0 e q}$. (Retaining the variation of height in the denominator does not greatly alter the solution and makes computation more difficult.)

Nondimensionalizing by

$$
h_{0}=H h_{0 e q}, u_{0}=U u_{0 e q}
$$

equations (11) and (12) become:

$$
\begin{gathered}
1-H=p(U-1)^{2} \\
1-H=\frac{q}{U}-r
\end{gathered}
$$

where

$$
q=\frac{F \tau}{u_{0 e q}}, p=\frac{5}{18} \frac{u_{0 e q}^{2}}{g^{*} h_{0 e q}}, r=k \tau,
$$

with $q, p$, and $r$ all positive.

We are looking for solutions of these two equations for which $U<1$ and $0<H<1$, since our analytical model requires that $u_{0}<u_{0 e q}$ and $0<h_{0}<h_{0 e q}$. Equilibrium solutions are solutions of the cubic equation

$$
U^{3}-2 U^{2}+U\left(1+\frac{r}{p}\right)-\frac{q}{p}=0
$$




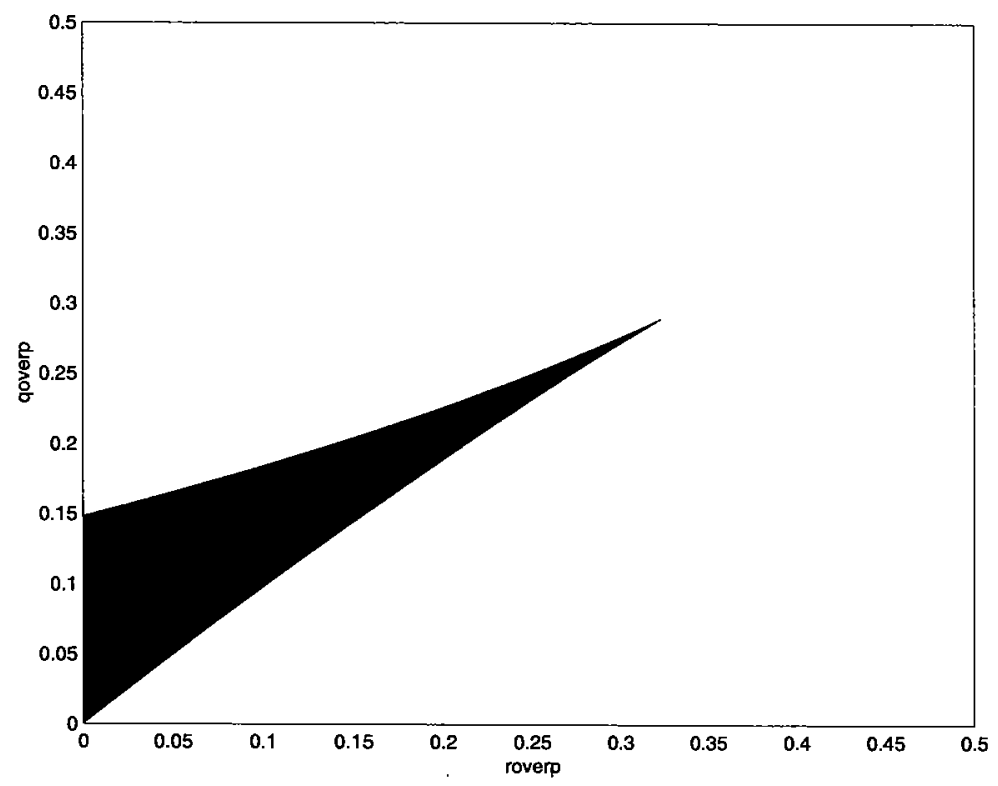

Figure 3: Region of multiple equilibria in analytical model. The dark region indicates values of $\frac{r}{p}$ and $\frac{q}{p}$ for which there exist multiple equilibria. $q$ corresponds to the strength of the applied forcing; $r$ corresponds to the friction; and $p$ relates to the Hadley circulation.

There are three real solutions when

$$
\left(-\frac{1}{9}+\frac{1}{3} \frac{r}{p}\right)^{3}+\left(-\frac{1}{27}-\frac{1}{3} \frac{r}{p}+\frac{1}{2} \frac{q}{p}\right)<0
$$

Otherwise, there is only one real solution, and we do not expect multiple equilibria in our model.

Figure 3 shows the parameter region where the simple model predicts multiple equilibria. In this region, the solution corresponding to the smallest $u$ is stable while the middle solution is unstable. Thus, model runs that start with initial conditions between the lowest and the middle solutions will equilibrate to the lower superrotating state. When the third solution (highest $u$ ) is a valid solution, we expect the system to go to this state if the initial conditions are above the middle solution. However, the solution with the highest $u$ is generally not valid because $u>u_{0 e q}$; in these cases, we expect that systems with initial conditions above the middle solution will equilibrate to a higher $u$ steady state, for which we do not have a model. The stability of the different equilibria can be verified using a potential function (Moehlis, personal communication).

We further examined the feedback in this simplified model. As the forcing increases, $u$ increases, which in turn decreases the Hadley circulation. Thus, less mass is brought up from below to decelerate the upper layer. This deceleration, $1-H$, can be thought of as a "damping", $r_{h}$, caused by the relaxation of the height to the equilibrium height. Rearranging equation (14), we obtain an expression for $U$ :

$$
U=\frac{q}{r_{h}(U(q))+r} .
$$


We want to know how $U$ changes with the forcing $(q)$ :

$$
\frac{\partial U}{\partial q}=\left(\frac{1}{r_{h}+r}\right) \frac{1}{1+\frac{q}{\left(r_{h}+r\right)^{2}} \frac{\partial r_{h}}{\partial U}}
$$

Using equation (13), which represents the Hadley circulation,

$$
\frac{\partial U}{\partial q}=\left(\frac{1}{p(U-1)^{2}+r}\right) \frac{1}{1+\frac{2 U(U-1)}{(U-1)^{2}+r / p}}
$$

When $\frac{\partial U}{\partial q}$ goes to infinity, $U$ must jump to a different solution. This quantity goes to infinity when

$$
U=\frac{2 \pm \sqrt{1-\frac{3 r}{p}}}{3}
$$

The lower value corresponds the the maximum $U$ of lower branch, while the upper value corresponds to minimum $U$ of upper branch. However, the upper branch is not well-modeled by the model because $U$ is usually greater than 1 . When $\frac{r}{p}>\frac{1}{3}, U$ never jumps to a different branch. When $r=0$ (i.e. frictionless case) there is an abrupt transition at $U=\frac{1}{3}$.

\subsection{Comparison of simple model and full model}

Figure (4) compares the $u$ fields from the full model and the simple model when there is no forcing. The computer model does not exactly match the simple analytical model because it does not conserve angular momentum. The flux of momentum from the lower layer decreases the angular momentum in the computer model. Note that this steady state was generated with $200 v$ grid points, while we searched for bifurcations using a grid of 50 $v$ points.

\section{Results}

For a given set of parameters, I ran the model until it came to a steady state (the change in $u$ from one time step to the next was less that some value). I started the run with one value of the forcing at the equator, $F_{0}$, and then used that final state as the initial conditions for the next run, which had a slightly higher forcing. $F_{0}$ generally ranged from 0 to $2 \times 10^{-6}$ $\mathrm{ms}^{-2}$ in steps of $10^{-8} \mathrm{~ms}^{-2}$. I ran the model again for the same forcings, this time starting at the highest forcing and decreasing the forcing each run. In this way, I was able to find multiple steady states for some ranges of forcings and parameters; however, this method can not find unstable equilibrium points.

Although the simple model does not depend on the shape of the forcing, the shape of the forcing affects the full model's behavior. The closeness of the full model to the analytical approximation depends on how well angular momentum is conserved by the full model near the equator. When forcing is only at the equator, it increases the wind at the equatorial grid point but has only indirect effects on the surrounding points. Thus, the winds immediately next to the equatorial point are less than the wind at the equator, and the approximation 


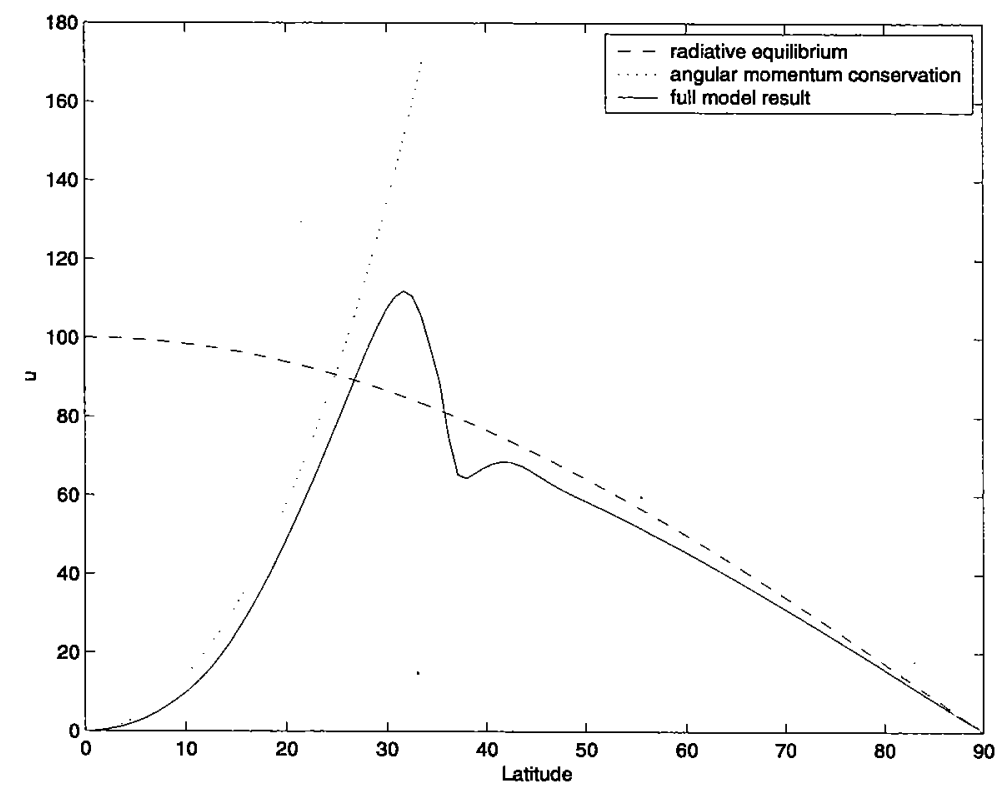

Figure 4: Comparison of zonal momentum from the simple model and the full model with no forcing. The solid line is the computational model result. The dotted line is $u$ from momentum conservation, and the dashed line is the radiative equilibrium result.

of angular momentum conservation starting from the equator is not valid. I could not find any bifurcations when forcing the system only at the equator.

However, once the forcing is spread out a bit, the tropics are closer to angular momentum conservation, and I was able to find multiple equilibria. In each run where I encountered multiple equilibria for some magnitude of the forcin, there were four regions of different behavior:

1. For small forcing, the system approximately agrees with the simple analytical model. The only stable solution is a Hadley-cell-like circulation with a small zonal velocity at the equator. The frictional term in the $u$ momentum equation at the equator is much less than the height relaxation term, which increases with increasing $F$. The dashed lines in Figures 5 through 7 show an example of the steady solution in this region.

2. For slightly higher forcing, the system has two steady solutions. One approximates the expected Hadley cell circulation, while the other has a higher $u$ and $h$. In the lower branch, relaxation back to equilibrium always dominates friction in the $u$ momentum equation at the equator, while in the upper branch, the two are the same order of magnitude. As $F$ increases along the lower branch, the relaxation term increases; however, on the upper branch, it decreases. The dash-dot lines in Figures 5 through 7 show an example of the steady lower branch solution while the dotted lines in Figures 5 through 7 show an example of the steady upper branch solution in this region.

3. When the forcing is further increased, the system has only one steady state. $u$ is generally above $u_{0 e q}$, but $h$ is always below $h_{e q}$. The relaxation term always decreases 


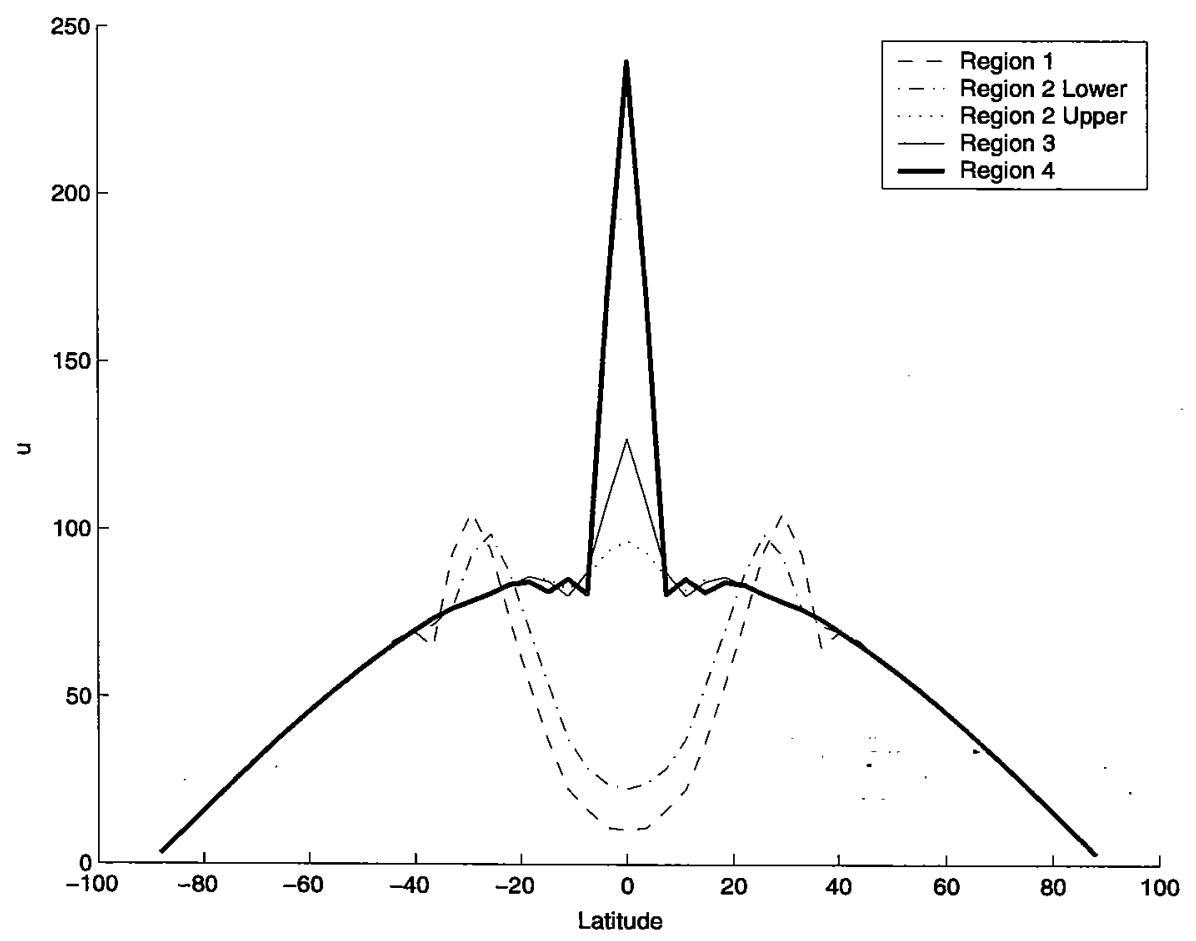

Figure 5: Steady state zonal velocity for $F_{0}=5 \times 10^{-7}$ (dashed), $8 \times 10^{-7}$ lower branch (dash dot), $8 \times 10^{-7}$ upper branch (dotted), $8.5 \times 10^{-7}$ (thin), $12 \times 10^{-7}$ (thick) for run 1 . 


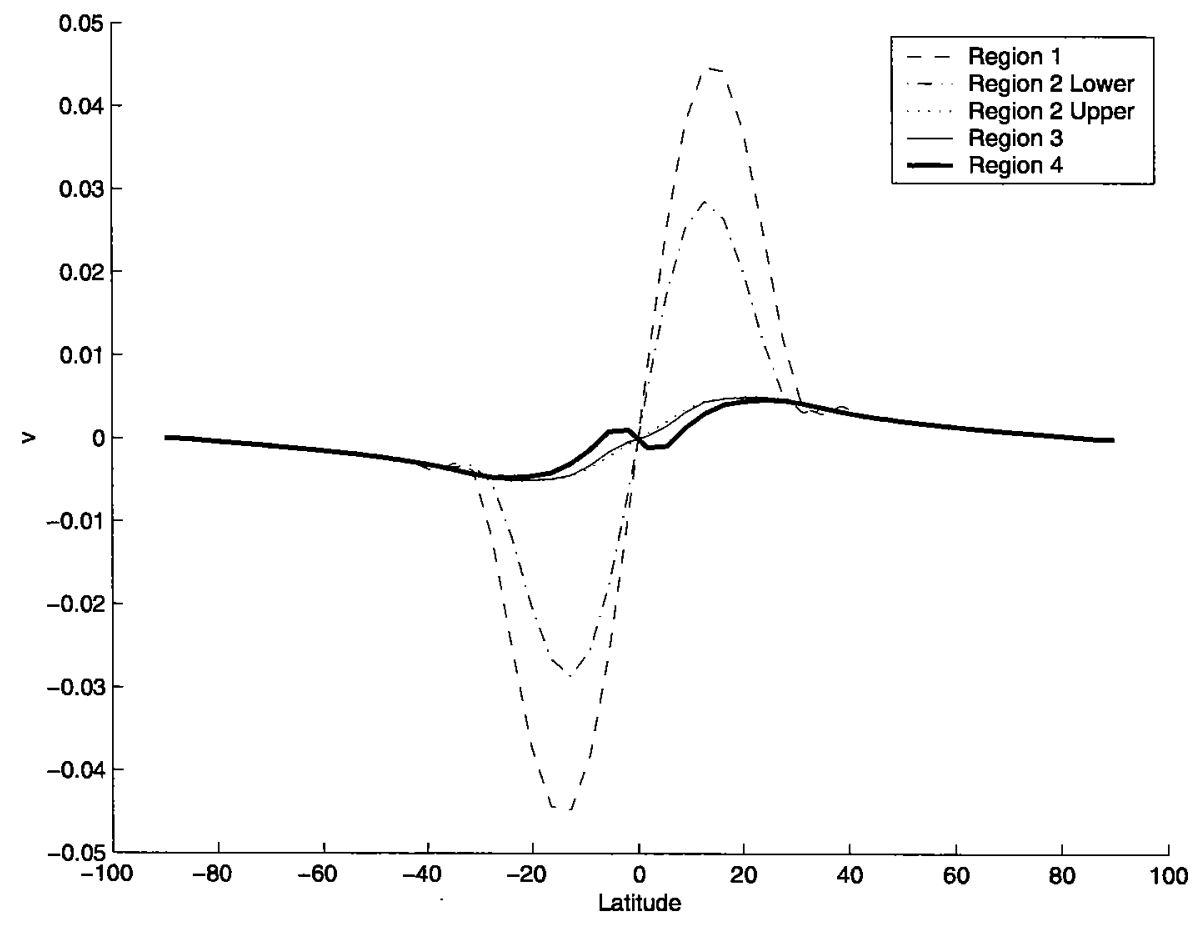

Figure 6: Steady state meridional velocity for $F_{0}=5 \times 10^{-7}$ (dashed), $8 \times 10^{-7}$ lower branch (dash dot), $8 \times 10^{-7}$ upper branch (dotted), $8.5 \times 10^{-7}$ (thin), $12 \times 10^{-7}$ (thick) for run 1 .

with increasing $F$, so the frictional term dominates by the high end of the region. The thin solid lines in Figures 5 through 7 show an example of the steady solution in this region.

4. For the highest values of the forcing, $h_{0}$ is above $h_{0 e q}$. Thus the only term in the equatorial zonal momentum equation which can balance the forcing term is the friction term, and $u_{0}$ has only one possible value:

$$
u_{0}=\frac{F}{k} \text {. }
$$

The thick solid lines in Figures 5 through 7 show an example of the steady solution in this region.

The size and location of the regions varied from run to run. The base run, run 1, used the values given in section $2, n=30$, and $50 v$ grid points. Table 1 describes how the other runs differed from run 1 and the ranges of the various regions. Table 2 describes the nondimensional parameters for each run and compares the predicted critical $u, u_{c}$ on the lower branch to the result obtained in the full model. As expected, $c_{r}$ was less than predicted in all cases, since the model was not run at exactly the critical forcing magnitude.

Figures 8 through 10 show the values of $u$ and $h$ for the different values of the forcing. The top figure shows how $U$ changes with the forcing. The middle figure shows how $1-H$ changes. In the bottom figure, the steady states for the different values of forcing are 


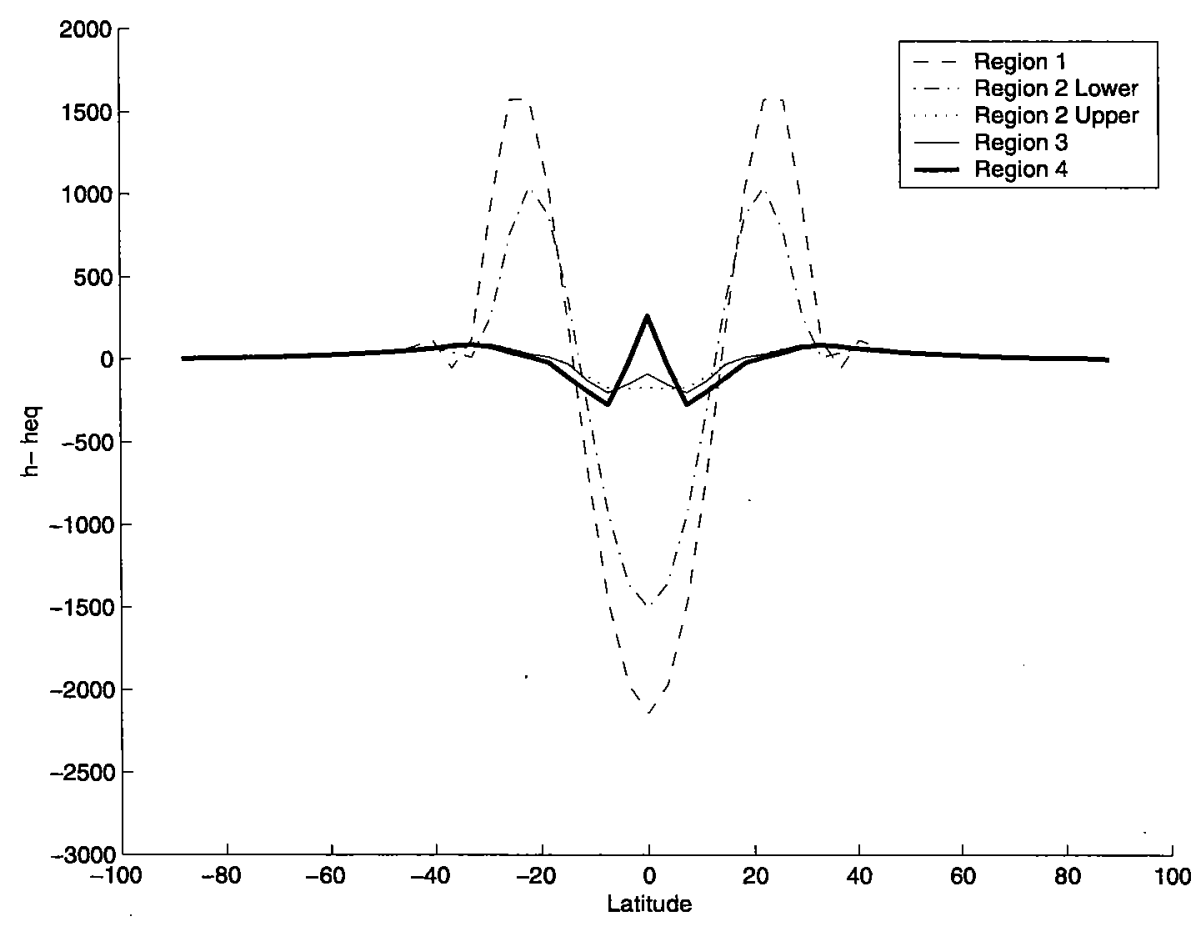

Figure 7: Steady state difference between layer height and relaxation height for $F_{0}=5 \times 10^{-7}$ (dashed), $8 \times 10^{-7}$ lower branch (dash dot), $8 \times 10^{-7}$ upper branch (dotted), $8.5 \times 10^{-7}$ (thin), $12 \times 10^{-7}$ (thick) for run 1 .

plotted along with the lines predicted by the simple model. The solid line corresponds to equation (13). Points for which the simple analytic model of the Hadley cell is a good approximation of the full model lie along this line. As $F_{0}$ is changed in a run, the line for equation (14) moves up and down. Where the two lines intersect, we expect a steady state. The dotted line corresponds to equation (14) for the maximum predicted multiple equilibria $F_{0}$, and the dashed line corresponds to the minimum predicted multiple equilibria $F_{0}$.

For the first three runs, $q=9000 * F_{0}, p=0.0500, r=0.0045$. From the simple model, we expect multiple equilibria in the range $F_{0}=4.9 \times 10^{-7}$ to $10.0 \times 10^{-} 7$. (In addition, the simple model has three valid solutions for the range $F_{0}=4.9 \times 10^{-7}$ to $5.0 \times 10^{-7}$.) These three runs all have multiple steady states for some portion of the range expected by the simple model. One run has multiple steady states for a slightly higher value than expected.

The first three runs study the effect of the shape of $F$ on the full model. The location of the multiple steady equilibria region seems to be within or close to the range predicted by the simple analytical model. However, the range is always less than that predicted by the simple model. The region of multiple equilibria seems to be related to where the model closely conserves angular momentum in the tropics.

Run 4 showed no multiple steady state region, though there is an abrupt transition when $F_{0}=6 \times 10^{-7}$, so that may be some very small region of multiple equilibria at this transition point. For this run, $q=9000 * F_{0}, p=0.025$, and $r=0.0045$. Thus, we expect multiple equilibria in the range $F_{0}=4.7 \times 10^{-7}$ to $6.0 \times 10^{-7}$. (The simple model has three 


\begin{tabular}{clccccc}
\hline Run & Description & 1 & 2 & predicted 2 & 3 & 4 \\
\hline 1 & base run & $5-7.0$ & $7.1-8.4$ & $4.9-10.0$ & $8.5-8.9$ & $9.0-15$ \\
2 & $\mathrm{n}=50$ & $3-9.5$ & $9.6-10.3$ & $4.9-10.0$ & $10.4-12.3$ & $12.4-20$ \\
3 & $\mathrm{~F}_{2}, \mathrm{n}=9$ & $3-8.3$ & $8.4-9.6$ & $4.9-10.0$ & $9.7-12$ & $? ? ? ? ?$ \\
4 & $\Omega=14.584 \times 10^{-5}$, & $1-6.0$ & - & $4.7-6.0$ & $6.1-7.7$ & $7.8-10$ \\
& $h_{0 e q}=113264$ & & & & & \\
5 & $h_{0 e q}=113264$ & $1-6.4$ & - & $4.7-6.0$ & $6.5-9$ & 10 \\
6 & $k=1 \times 10^{-8}$ & $1-12$ & - & $9.5-12.1$ & $13-18$ & $19-20$ \\
\hline
\end{tabular}

Table 1: Ranges of $F_{0}$ for the different solution regions. The values are in $10^{-7} \mathrm{~ms}^{-2}$.

\begin{tabular}{cccccc}
\hline Run & $p$ & $r$ & $q$ & predicted $u_{c}$ & $u_{c}$ \\
\hline 1 & 0.05 & 0.0045 & $9000 \times F_{0}$ & 0.3819 & 0.276 \\
2 & 0.05 & 0.0045 & $9000 \times F_{0}$ & 0.3819 & 0.352 \\
3 & 0.05 & 0.0045 & $9000 \times F_{0}$ & 0.3819 & 0.306 \\
4 & 0.025 & 0.0045 & $9000 \times F_{0}$ & 0.4406 & 0.351 \\
5 & 0.025 & 0.0045 & $9000 \times F_{0}$ & 0.4406 & 0.367 \\
6 & 0.05 & 0.009 & $9000 \times F_{0}$ & 0.4406 & 0.303 \\
\hline
\end{tabular}

Table 2: Predicted critical $u$ on the lower branch and maximum $u$ obtained on the lower branch of the full model. For cases that do not bifurcate, $u_{c}$ corresponds to where the height relaxation term transitions from increasing to decreasing with increased forcing.

valid solutions for the range $F_{0}=4.7 \times 10^{-7}$ to $5.0 \times 10^{-7}$.) The system transitions to region 3 at the high end of the multiple steady state forcing region.

For Run 4, I increased $h_{0 e q}$ along with $\Omega$ to prevent the height of the layer from becoming negative. To see the effect of the height alone, Run 5 uses the normal rotation rate but the higher layer height. This run does not even seem to have an abrupt transition; it changes gradually from weakly to strongly superrotating. The values of $p, q$, and $r$ are the same as for Run 4 , since $\Omega$ does not affect the simple model (other than determining the critical latitude).

Since friction plays an important role in this system (it affects the locations of the equilibria of the model and becomes important to the behavior of system in regions 3 and 4), I doubled the friction for Run 6. Thus, the parameters for this run are $q=9000 \times F_{0}$, $p=0.05$, and $r=0.009$, and the simple model predicts multiple equilibrium between $F_{0}=9.5 \times 10^{-7}$ and $12.1 \times 10^{-7}$, with three valid solutions for the range $F_{0}=9.5 \times 10^{-7}$ to $10.0 \times 10^{-} 7$. I found no region of multiple equilibria, and the steady state value of $u$ does not show any abrupt changes.

\section{Rossby waves}

We attempted to add the effects of breaking Rossby waves to the full model. At each time step, Rossby waves with a certain distribution of phase speeds and pseudo-angular- 

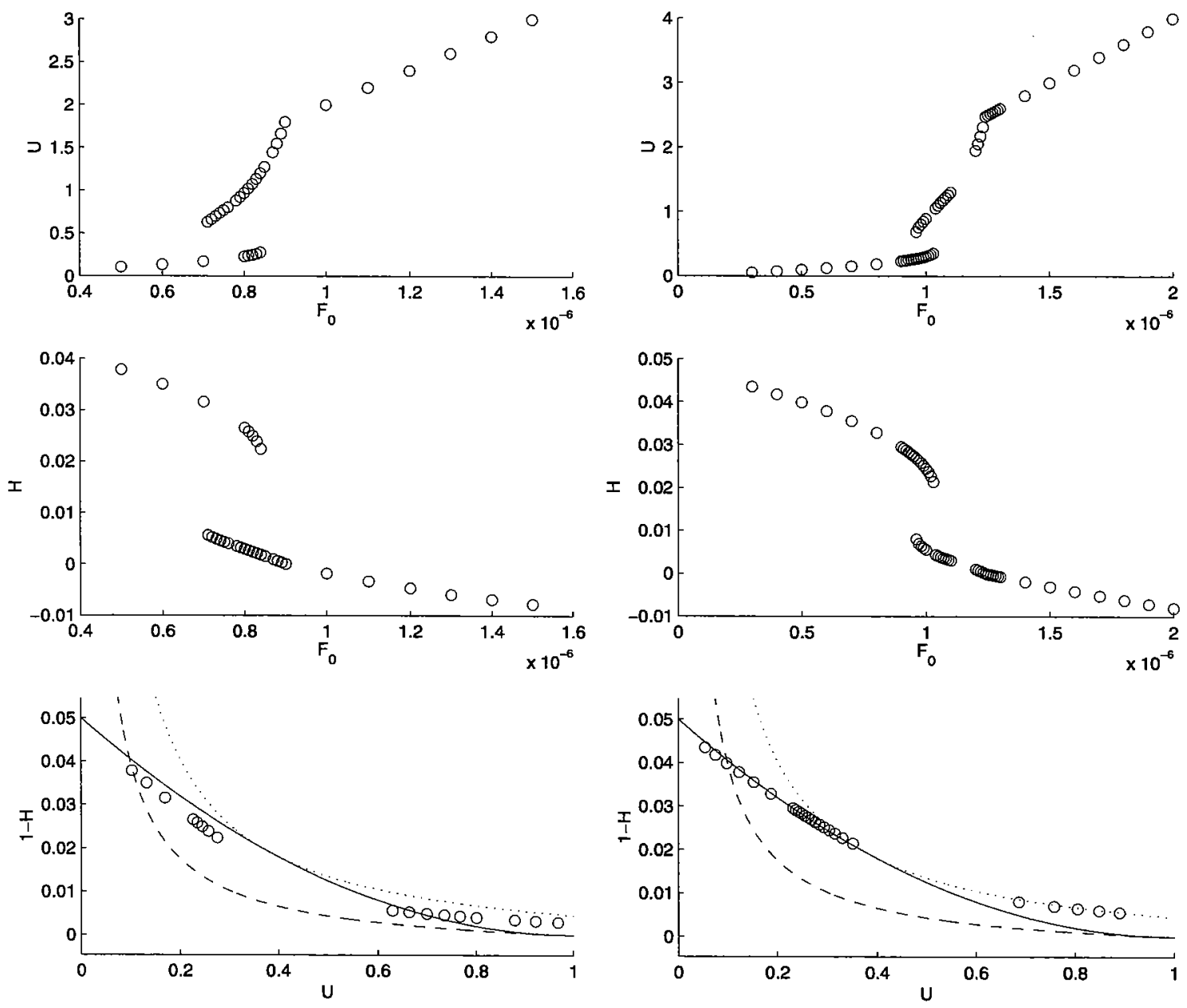

Figure 8: Runs 1 (left) and 2 (right). See text for explanation.

momentum were introduced at a certain latitude. These Rossby waves moved towards the equator (and across to the other hemisphere, if necessary) and "broke" at the first latitude where their angular phase speed was greater than or equal to the angular momentum of the zonal winds. The breaking waves decreased the angular momentum at that latitude and thus reduced the zonal winds.

I used wave speeds, $c$, distributed between 5 and $45 \mathrm{~m} / \mathrm{s}$. The distribution of angular pseudomomentum deposited when waves of a particular speed break, $P(c)$ is

$$
P(c)=\frac{P_{\max }}{h \Delta \phi \cos \phi} e^{-\left(\frac{c-25}{10}\right)^{2}}
$$

The addition of the waves allows for the possibility of periodic solutions. The waves break at a certain latitude, which slows down the flow there (and through advection, surrounding areas). Thus, the waves start breaking earlier (i.e. further poleward) and decreasing the flow there. The waves continue breaking earlier and earlier until they get to the 

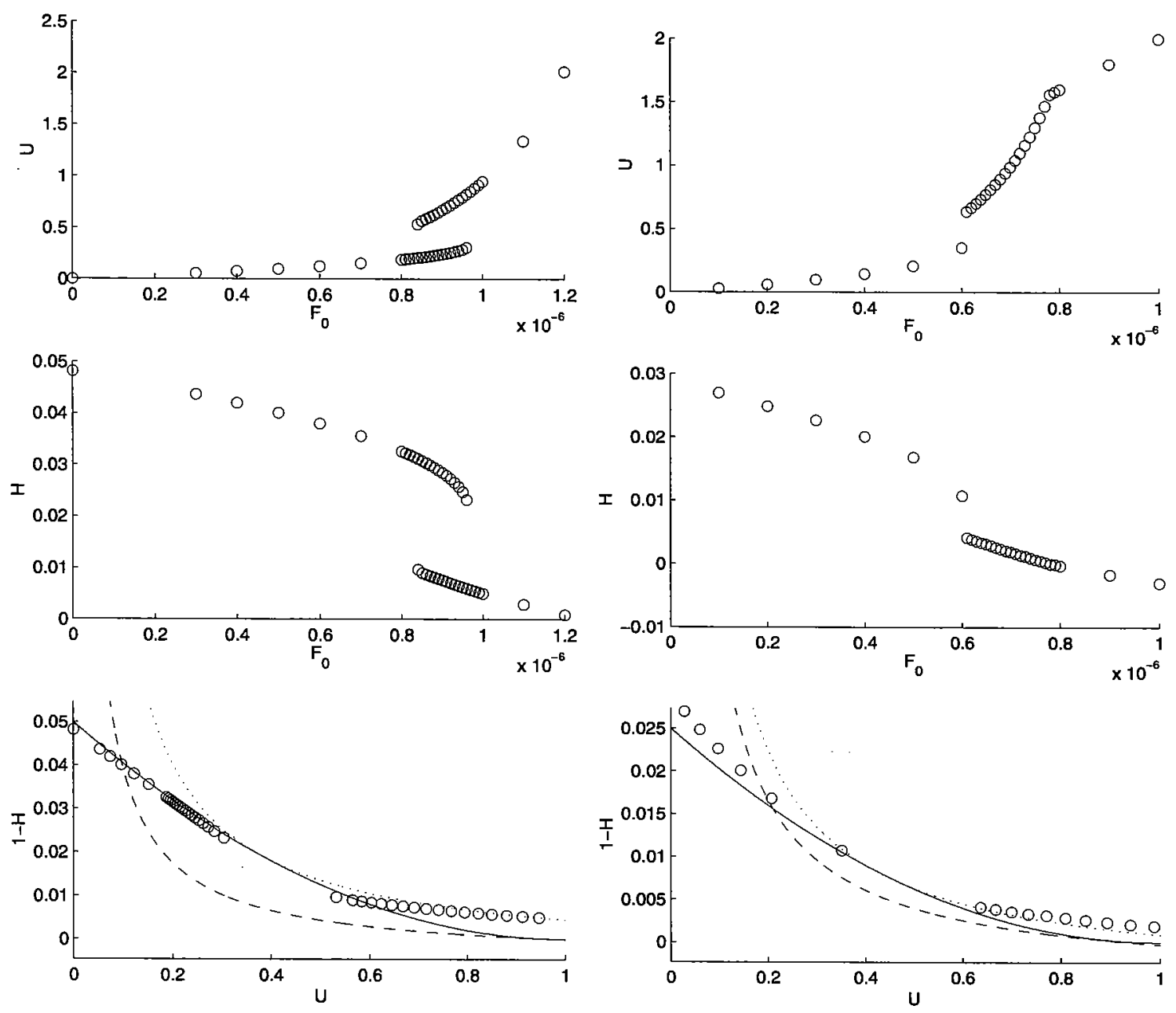

Figure 9: Runs 3 (left) and 4 (right). See text for explanation.

jet. Eventually, the disturbance moves to a region where $u$ is high enough that some of the lower phase speed waves can pass through to the equatorial region. Another disturbance starts before the first one gets to the jet.

I studied the behavior of the waves with no forcing. The system reached a stable periodic solution. However, the period of the solution varied with the grid resolution. Thus, we did not pursue this parameterization further.

\section{Conclusion}

It is possible to get bifurcations in the superrotation strength in an axisymmetric model for some parameter ranges. When bifurcations exist, the stable equilibria lie along two branches of $u$ values as the forcing is changed. On the lower branch, damping due to height relaxation increases with increasing $F$; on the upper branch, the damping decreases with increasing $F$. 

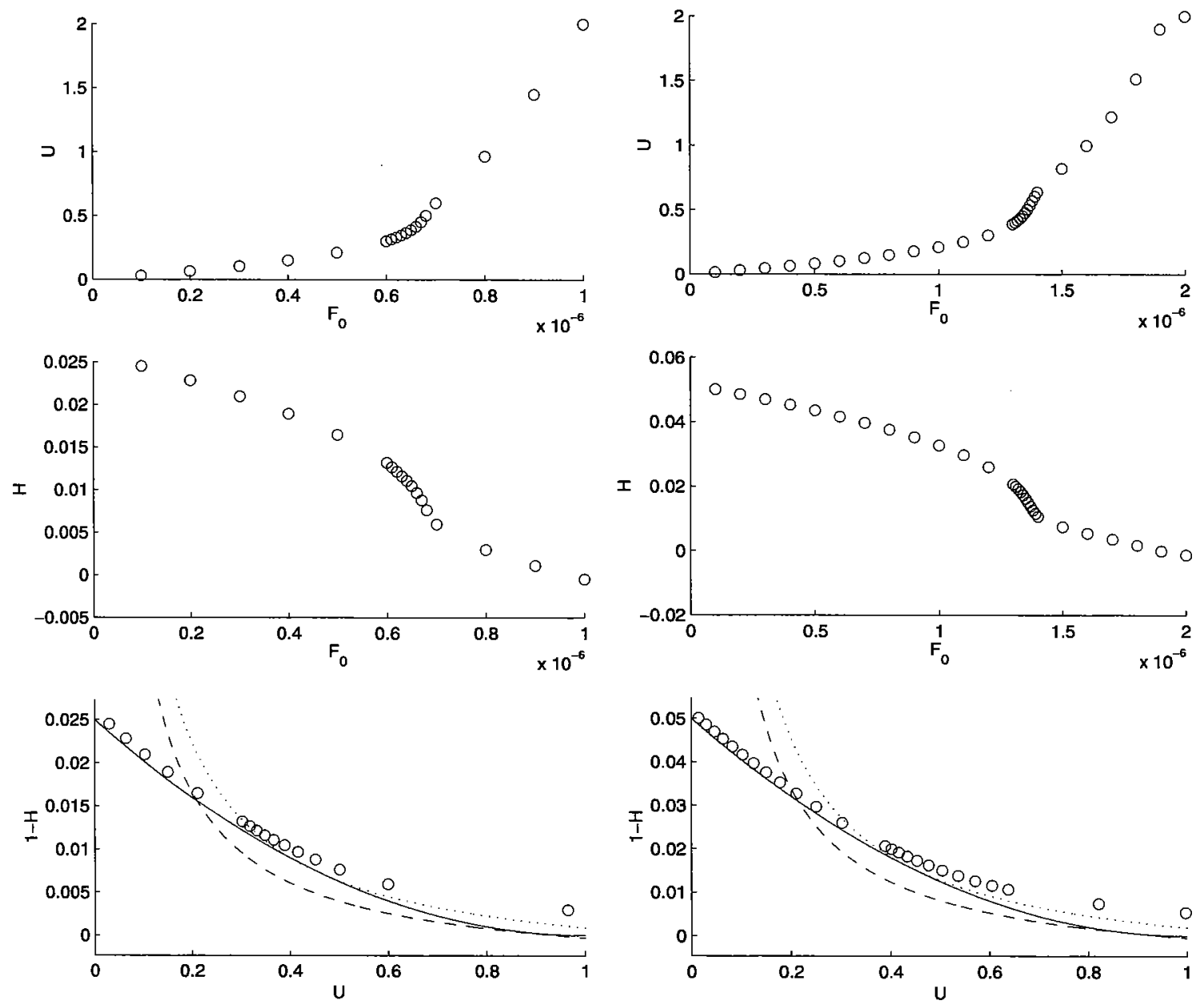

Figure 10: Runs 5 (left) and 6 (right). See text for explanation.

Although the simple model approximately predicts where the full model will have multiple equilibria, the range is smaller than predicted and sometimes multiple equilibria are not present at all. The presence and location of the bifurcation has something to do with how well angular momentum is conserved in tropics, which depends on forcing. However, the exact relationship is not clear.

\section{Acknowledgments}

I would like to thank my project supervisor, Isaac Held, for his help with all aspects of my project. I would also like to thank Jeff Moehlis for help with the nonlinear dynamics and for his general enthusiasm for the project, which increased my own. Thanks to Giulio for model discussions. Thanks to Cheryl and Tevon for providing the music and everyone who went dancing with me for keeping me sane. Thanks to Chris and his green car for providing the transportation. GFD is fun and easy. 


\section{References}

[1] M. J. Suarez and D. G. Duffy, "Terrestrial superrotation: A bifurcation of the general criculation," J. Atmos. Sci. 49, 1541 (1992).

[2] R. Saravanan, Ph.D. thesis, Princeton University, Princeton, NJ 08544, 1990.

[3] H.-P. Huang, K. M. Weickmann, and C. J. Hsu, "Trend in atmospheric angular momentum in a transient climate change simulation with greenhouse gas and aerosol forcing," J. Clim., submitted.

[4] I. M. Held, "Equatorial superrotation in earth-like atmospheric models," Bull. Am. Met. Soc., in preparation.

[5] I. M. Held and A. Y. Hou, "Nonlinear axially symmetric circulations in a nearly inviscid atmosphere," J. Atmos. Sci. 37, 515 (1980). 


\title{
What Makes Ocean Gravity Currents Flow Downhill?
}

\author{
Christopher C. Walker
}

\section{Introduction}

Gravity currents are the flow of one fluid through another as a result of a density contrast between the two fluids. The density contrast between the fluids can be caused by different mechanisms, for example, salinity contrasts, temperature differences, and sediment load differences. Gravity currents are common in geophysical fluid dynamics and occur on a large range of spatial and temporal scales. In the atmosphere, downslope (katabatic) winds, sea breeze fronts, and thunderstorm downdrafts are all gravity currents that are attributed to temperature differences between ambient air and cooler intruding air. Atmospheric gravity currents are also driven by differences in sediment load: avalanches and volcanic surges are both gravity currents driven by the presence of suspended particles[1].

In the ocean, gravity currents play an important role in the large-scale circulation by acting as a conduit for deep water formation. In this case, water in a marginal sea (an area of the ocean that is isolated from the rest of the ocean by topography) is subjected to sustained cooling or evaporation, and the resulting cold, dense water sinks to the bottom of the marginal sea. Once at the bottom, the dense water flows out (as a so-called "outflow") of the marginal sea, continuing down the continental slope into the deep ocean[2].

Several models have been put forth to explain the path of oceanic outflows as they propagate down continental slopes. The simplest of these models treat outflows as frictionless, non-entraining, and steady flows. Under these conditions, geostrophic balance and the conservation of both potential vorticity and mass dictate that the current flow along a path of constant topography[3]. In this simplified model, the path and velocity of the flow are determined by the density difference between the two fluids, the slope of the incline, the Coriolis parameter, and the mass flux of the current. More complicated models of outflows, such as the streamtube models devised by Smith[4], and later extended by Price and Baringer[2], include parameterizations of bottom drag and entrainment of the ambient fluid. When friction is included in the model, the path of the current is no longer restricted to following lines of constant depth; instead, with friction present, a steady current is free to cross lines of constant depth.

Although streamtube models can be tuned to give good agreement with observations, it is clear that these models are missing some important aspects of outflows. First, streamtube models assume that the ambient fluid is quiescent, eliminating potentially important interactions between the gravity current and the surrounding fluid. Second, streamtube models are a measure of the bulk properties of the flow; no cross-stream variations are considered 
in a streamtube model. Third, and most relevant to this study, streamtube models assume that the flow is steady; in a streamtube model the flux in the outflow is assumed to be constant in time.

More complicated numerical models of oceanic outflows have recently been applied to address some of the shortcomings of streamtube models. Jungclaus and Backhaus[5] developed a hydrostatic, reduced gravity, two-dimensional primitive equation model and applied it to a study of the Denmark Strait outflow. They demonstrated that the presence of bottom topography can cause complicated cross-stream variations in the flow. Subsequent numerical studies by Jiang and Garwood $[6,7]$ considered outflows in the context of a three dimensional ocean model. Their results revealed that the three dimensional aspects play an important role in the dynamics of the outflow. Specifically, they showed that the outflow plume can separate into smaller sub-plumes, and that as the plume propagates it can manifest itself as coherent vortices in the ambient fluid. This behavior was later observed and explored in laboratory experiments $[8,9]$.

In contrast with outflows, turbidity currents are an example of a transient oceanic gravity current. Turbidity currents arise when sediment on the continental slope is dislodged, often by an underwater earthquake. Once dislodged, the sediment is brought into suspension, increasing the density of the fluid in the immediate vicinity of the earthquake. This dense fluid then flows down the continental slope, entraining more sediment as it travels. The turbidity current eventually encounters a decreasing slope, slowing the current and allowing the suspended particles to settle. Large-scale turbidity currents have not been directly observed. There is, however, one turbidity current that gives a unique set of data for analysis. In 1929, an underwater earthquake occurred under the Grand Banks of Newfoundland, causing a large turbidity current that flowed down the continental slope. As this current traveled, it broke a succession of telegraph cables, each of which had a known location. The time of each breakage was also well known, giving a measure of the current's speed[10]. Subsequent investigation determined the extent of the turbidite (the material deposited by the current), providing data about the path that the turbidity current traveled down the continental slope. The turbidite indicates that the turbidity current veered to the right as it flowed, possibly under the influence of the Coriolis force.

The outflow models discussed above all model oceanic gravity currents as steady flows. While steady flow is a reasonable assumption for outflows, it is not a reasonable assumption for turbidity currents, which are a much more transient event. The only work that we are aware of that studies the effect of rotation on turbidity currents is a study by Nof[10] (hereafter referred to as Nof), which proposes a simple model (discussed more in section 4.1) of the turbidity current that resulted from the 1929 Grand Banks earthquake. Nof's model was intended to explain a slowing down of the current that was seen in the cable breakage data. As the turbidity current propagated down the continental slope, its downhill velocity decreased. Previous work attributed this slowing to a combination of three causes. First, as the current propagated, it experienced a decrease in slope which acted to slow the current. Second, as the current slowed, sediment settled out of solution and decreased the density contrast between the plume and the ambient fluid. Third, bottom friction between the slope and the plume acted to slow the plume. Nof proposed that in addition to the reasons listed above, the influence of the Coriolis force could have contributed to the decrease in downhill velocity by causing the current to veer to its right. To determine the 
effect of the Coriolis force on the turbidity current, Nof modeled the turbidity current as a frictionless "blob" of fluid on a parabolic bottom. The solution to Nof's model is a cycloid with a mean path parallel to the slope, showing qualitatively that the Coriolis force may have played a substantial role in reducing the current's downhill speed.

This work has two goals. First, it is designed as a modeling complement to Nof's analytic study on the role of rotation in the path of turbidity currents. Second, we wish to address the question, "If, as predicted by streamtube models, an inviscid and geostrophic gravity current propagates along contours of constant topography, under what conditions will a gravity current flow downhill?"

An outline of this paper is as follows: In section two, we outline the approach that we use to address the question raised above. In section three, we discuss a simple outflow model that is based on Smith's streamtube model. Section three also includes comparisons of our model results with the streamtube theory. In section four, we review the model proposed by Nof[10] to explain the path of the 1929 Grand Banks turbidity current and discuss the results of our model of a turbidity current. Section five summarizes our results and answers the question raised above about the paths of oceanic gravity currents.

\section{Approach}

To address the question of when gravity currents flow downhill, we start by considering the horizontal momentum equation in natural coordinates ( $t$ is the unit vector parallel to the flow, $n$ is the unit vector normal to the flow). When the flow is in steady state, it is parallel to height contours and the velocity statisfies[11]:

$$
\frac{V^{2}}{R}+f V+\frac{\partial \Phi}{\partial n}=\frac{C_{d}}{H} V^{2}
$$

where $V$ is the horizontal velocity, $R$ is the radius of curvature of the flow, $f$ is the Coriolis parameter, $H$ is the height of the Ekman layer (assumed to have a maximum value of $10 \mathrm{~m}$ ), $C_{d}$ is a dimensionless bottom friction parameter, and $\Phi$ is the geopotential. The term on the right represents a height dependent boundary layer friction, so that equation (1) can be thought of as representing the momentum balance in an inviscid layer of fluid with a frictional bottom boundary layer of height $H$, where $H$ has a maximum value of $10 \mathrm{~m}$. For large scale flows, the term in the momentum equation representing the centrifugal force $\left(\frac{V^{2}}{R}\right)$ is relatively small and can be neglected. When the centrifugal term is neglected and boundary layer friction is ignored, the motion is a balance between the Coriolis force and the pressure gradient force, so that the motion is geostrophic:

$$
V_{g}=-\frac{R}{f} \frac{\partial \Phi}{\partial n}
$$

where $V_{g}$ is the geostrophic velocity. Although the geostrophic approximation is appropriate for most large-scale flows, there are regimes in which the centrifugal force cannot be neglected. In these cases, the full momentum equation (1) must be considered. The Rossby number, the ratio of the centrifugal term to the Coriolis term, gives the degree of 
appropriateness of the geostrophic approximation:

$$
R_{o}=\frac{V}{f L}
$$

Flows with a low $(\ll 1)$ Rossby number can be considered geostrophic; flows with a Rossby number near 1 must consider the full momentum equations. Another important dimensionless number is the Ekman number, which measures the relative importance of the friction term against the Coriolis term. In this case, the Ekman number is given by:

$$
E_{k}=\frac{C_{d} V}{f H}
$$

The Ekman number indicates the extent to which friction plays a role in the flow.

As mentioned in the introduction, a gravity current that is both inviscid and geostrophic will travel along a path that follows bottom topography. If a gravity current is to flow downhill, then one of these conditions must be relaxed. In this study, we use a simplified version of an ocean general circulation model to investigate situations under which these two approximations-inviscid flow and geostrophy-are no longer applicable.

We consider two flow regimes. First, a regime in which the flow is approximately in geostrophic balance, but is modified by bottom boundary friction. This describes the conditions associated with a midlatitude $\left(f=10^{-4} \mathrm{~s}^{-1}\right)$ outflow, since the velocities of an outflow are relatively small $\left(\approx 40 \mathrm{~cm} \mathrm{~s}^{-1}\right)$ and the length scales are large $(\approx 40 \mathrm{~km})$, giving a Rossby number on the order of 0.1 . The same scales, together with a layer height of 10 $\mathrm{m}$ and $\mathrm{C}_{d}=0.005$, give an Ekman number of approximately 0.5 , indicating that friction will play a substantial role in the motion of the plume. We also consider the behavior of an overflow at lower latitudes $\left(f=5 \times 10^{-5} \mathrm{~s}^{-1}, f=10^{-5} \mathrm{~s}^{-1}\right.$ and $\left.f=0 \mathrm{~s}^{-1}\right)$, so that we have flows with Rossby numbers from $R_{o}=\infty$ to $R_{o}=0.1$.

The second regime that we study is a regime in which the full equation (1) must be considered. This regime describes our model of a turbidity current, since the velocities are large $\left(\approx 10 \mathrm{~m} \mathrm{~s}^{-1}\right)$, the height $H$ is $10 \mathrm{~m}$, and the length scales are on the order of $200 \mathrm{~km}$. These scalings give a Rossby number of 0.5 and an Ekman number of 50, indicating that all of the terms in (1) will play a significant role in the motion of the current.

\subsection{Methods}

To consider the two regimes described above, we use the Miami Isopycnal Coordinate Ocean Model (MICOM) to simulate some basic aspects of oceanic gravity currents. A full description of MICOM is beyond the scope of this work; a complete discussion of MICOM can be found in Haidvogel and Beckmann[12] and references therein. MICOM is a threedimensional ocean model with potential density as the vertical coordinate. To study gravity currents, we run the model in a two-layer configuration: one layer representing the upper ambient fluid and a heavier layer representing the intruding gravity current. We run the model without entrainment, so that the mass in each layer remains constant.

To isolate fundamental processes in the dynamics of gravity currents, we consider two simple model configurations, one configuration designed to study outflows, and the other designed to study turbidity currents. In both configurations, we consider the motion of a plume on an $f$-plane. 
To study outflows, we set up the model in a configuration similar to Jungclaus and Backhaus, i.e. a $320 \times 320 \mathrm{~km}$ model domain with a $2 \mathrm{~km}$ horizontal resolution. Heavy fluid $\left(\Delta \rho / \rho=2.9 \times 10^{-4}\right)$ is released at the top of the slope, and the model is integrated for five model days. To test the dependence of the solution on the initial conditions, we release the fluid in two different manners: a "dambreak" configuration in which the heavy fluid starts as a bay (horizontal size of $30 \times 30 \mathrm{~km}, 100 \mathrm{~m}$ height) of fluid at the top of the slope and is allowed to evolve with no subsequent addition of fluid. In addition to the dambreak release, we also release fluid as a constant flux at the top of the slope. There is little qualitative difference in the behavior of the plume in between the two cases; accordingly, only the results from the constant flux configuration are discussed here.

Our second configuration is designed to test Nof's model of turbidity currents (discussed in section 4.1). Because the spatial scales of turbidity currents are large, we consider a 1000 $\times 1000 \mathrm{~km}$ model domain, with a $5 \mathrm{~km}$ horizontal resolution. Rather than release dense fluid at the top of the slope as we did in the outflow configuration, we initialize the model with a Gaussian blob (radius $\approx 100 \mathrm{~km}$, maximum height of $15 \mathrm{~m}$ ) of dense fluid $(\Delta \rho / \rho=0.03$ ) near the top of the slope. Initializing the model in this manner is based on the approach taken by Nof. To make the study of turbidity currents mathematically tractable, Nof assumes that the early behavior of the turbidity current is not important to its path. Accordingly, Nof considers the fate of a geostrophically adjusted "blob" of fluid on a slope, which is taken to represent the turbidity current after the complex initial stages. To facilitate comparison with Nof's theory, we take the same approach, although we also considered the behavior of a turbidity current starting from a dambreak configuration. We found little difference between the two initial configurations and therefore report only the results obtained with the Gaussian blob configuration.

\section{Outflow regime}

\subsection{Limiting case of Smith's streamtube model}

In the streamtube model formulated by $\operatorname{Smith}[13,4]$, the outflow is treated as a steady flow on a simple linear slope. The streamtube model starts with the hydrostatic, incompressible Navier-Stokes equations and considers the cross-stream properties of a "tube" of fluid". With the simplifying assumptions made by Smith, and by neglecting entrainment into the plume and stratification of the ambient fluid, the motion of the flow can be reduced to a set of two ordinary differential equations:

$$
\begin{gathered}
V\left(f+V \frac{d \beta}{d \xi}\right)=g^{\prime} \alpha \cos \beta \\
\frac{d\left(A V^{2}\right)}{d \xi}=g^{\prime} \alpha A \sin \beta-C_{d} W V^{2}
\end{gathered}
$$

where $\xi$ is a coordinate that is along the path of the flow, $\alpha$ is the slope of the topography, $\beta$ is the angle that the streamtube makes with a line running parallel to topography, $V$ is the mean velocity of the flow, $A=H W$ is the cross-sectional area of the flow, where $H$

\footnotetext{
${ }^{1}$ The details and derivation of the streamtube model can be found in [13]
} 
and $W$ are the height and width of the streamtube, respectively, and $\mathrm{C}_{d}$ is a parameter taken to represent friction from bottom drag. Note that $\mathrm{C}_{d}$ has the same meaning as it has in equation (1), and that it has been multiplied by the width of the plume because of the cross-stream integration performed as part of the streamtube analysis.

When the full streamtube equations are integrated numerically, the motion of the plume is initially a cycloid with a mean path along the slope. Far downstream, friction damps out any cyclic component of the flow, and the flow settles into a steady state such that $\frac{d}{d \xi}=0$. At this limit, equations (5) and (6) can be combined to give:

$$
\begin{gathered}
\tan \beta=\frac{C_{d} V}{f H} \\
\left(\frac{C_{d}}{H f}\right)^{2} V^{4}+V^{2}=\left(\frac{g^{\prime} \alpha}{f}\right)^{2}
\end{gathered}
$$

Note that the above expression for $\tan \beta$ is similar to the expression for the Ekman number (4). The only difference between the two expressions is that in the definition of the Ekman number, $H$ corresponds to the height of the mixed layer and therefore has a maximum value of $10 \mathrm{~m}$. In equation (7), $H$ is the height of the streamtube, with no maximum value (although the height of the flow is generally less than $100 \mathrm{~m}$ ). In spite of this difference, we refer to the right hand side of equation (7) as the Ekman number $\left(E_{k}\right)$. As defined above, $\beta$ is the angle that the streamtube makes with a line running along the topography. The expression for $\tan \beta$ is therefore the ratio of the velocity across the topography to the velocity along the topography, or

$$
\tan \beta=E_{k}=\frac{V_{\text {across }}}{V_{\text {along }}}
$$

The solution to equations (7) and (8) is most useful when it is written in terms of external variables so that the path and velocity of the streamtube can be predicted. As in Price and Baringer[2], we assume that the width of the plume is constant so that the volume flux per unit width, $Q$, is written as $Q=V H$. Substituting this expression into equations (7) and (8) gives:

$$
\begin{gathered}
\tan \beta=\frac{C_{d} V^{2}}{f Q} \\
\left(\frac{C_{d}}{Q f}\right)^{2} V^{6}+V^{2}=\left(\frac{g^{\prime} \alpha}{f}\right)^{2}
\end{gathered}
$$

\subsection{Comparison with model results}

We consider two methods of solving equations (10) and (11), which we designate Method 1 and Method 2. Each method has an associated velocity (which we denote as $V_{1}$ and $V_{2}$ ), and an associated $\beta\left(\beta_{1}\right.$ and $\left.\beta_{2}\right)$.

The first method, Method 1, considers the simultaneous solution to equations (10) and (11). The solution to equation (11) has only one positive real root $\left(V_{1}\right)$, which is substituted in (10) to obtain $\beta_{1}$. 
The second method of solving equations (10) and (11)-the approach taken by Price and Baringer[2]-is to assume that the flow is in geostrophic balance. Method 2 does not make use of the predictive expression for the velocity, equation (11); instead, it assumes that the velocity, $V_{2}$, is given by geostrophic velocity, $U$. The geostrophic velocity for flow on a constant linear slope is given by

$$
V_{2}=U=\frac{g^{\prime} \alpha}{f}
$$

When (12) is substituted into (10), the following expression results:

$$
\tan \beta_{2}=\frac{C_{d}\left(\alpha g^{\prime}\right)^{2}}{Q f^{3}}
$$

To test the predictive capabilities of the two methods of solving the streamtube equations and to observe the sensitivity of the outflow path to different parameters, we integrated the model with different rotation rates and different values of bottom friction. The path of the outflow for different rotation rates is seen in Fig. 1, which shows the height (thin contours) of the plume ${ }^{2}$ on day five of the integration, for four different rotation rates. Each panel in Fig. 1 also shows the path predicted by the two different methods of solving the streamtube equations. Method 1, which is the simultaneous solution to equations (10) and (11), is shown as a heavy solid line. Method 2, which assumes that the plume is in geostrophic balance, is shown as a heavy dashed line. Dense fluid is released at a constant flux (per unit width) of $20 \mathrm{~m}^{2} \mathrm{~s}^{-1}$ near the top right corner of each panel between $210 \mathrm{~km}$ and $240 \mathrm{~km}$. To avoid the complications associated with flow near a boundary, the fluid is released on the slope $20 \mathrm{~km}$ from the top edge of the domain. The topography starts at the top of each panel with a value of $-500 \mathrm{~m}$ and declines linearly towards the bottom of each panel. In each case, the slope of the topography is 0.008 and bottom friction $\left(\mathrm{C}_{d}\right)$ is 0.01 . The rotation rate has values of $0 \mathrm{~s}^{-1}$ (upper left panel), $10^{-5} \mathrm{~s}^{-1}$ (upper right panel), $5 \times 10^{-5} \mathrm{~s}^{-1}$ (lower left panel), and $10^{-4} \mathrm{~s}^{-1}$ (lower right panel). As a complement to Fig. 1, Table 1 shows the values of velocity ${ }^{3}$ and Ekman number $(\tan \beta)$ obtained by the two different solution methods for the integrations shown in Fig. 1. Table 1 also shows the measured average velocity of the plume, where we define the average velocity of the plume as the instantaneous average velocity of all points that have a thickness of $10 \mathrm{~m}$ or more.

The effect of rotation is seen clearly in Fig. 1: as the rotation rate is increased, the path of the outflow is increasingly to the right (northern hemisphere configuration). For the case without rotation, the path is directly downhill. When the rotation rate is $10^{-4} \mathrm{~s}^{-1}$, which corresponds to a latitude of approximately $45^{\circ}$, the path is substantially deflected.

With some notable exceptions, the predictions given by the streamtube theory are in good agreement with the model results. Without rotation, the behavior of the streamtube equations is pathological because of the presence of $f$ in the denominator in equations (10) and (11). In the limit of very small $f$, however, the simultaneous solution to equations (10) and (11) (Method 1) gives a velocity of approximately $42.4 \mathrm{~cm} \mathrm{~s}^{-1}$ and a very large $\tan \beta$. These values are in accord with the measured velocity, $40.1 \mathrm{~cm} \mathrm{~s}^{-1}$, and an infinite $\tan \beta$. Method 2 is not applicable without rotation, since the geostrophic velocity approaches infinity as $f$ approaches 0 .

\footnotetext{
${ }^{2}$ We define the plume as the dense fluid with a height of $10 \mathrm{~m}$ or greater.

${ }^{3}$ Note that the table entry for $f=0 \mathrm{~s}^{-1}$ is the solution to (10) and (11) as $f \rightarrow 0$
} 


\begin{tabular}{|c|c|c|c|c|c|}
\hline & \multicolumn{2}{|c|}{ Method 1 } & \multicolumn{2}{c|}{ Method 2 } & Observed \\
\cline { 2 - 6 } & $\mathrm{V}_{1}\left(\mathrm{~cm} \mathrm{~s}^{-1}\right)$ & $\tan \beta_{1}$ & $\mathrm{~V}_{2}\left(\mathrm{~cm} \mathrm{~s}^{-1}\right)$ & $\tan \beta_{2}$ & $\mathrm{~V}\left(\mathrm{~cm} \mathrm{~s}^{-1}\right)$ \\
\hline $\mathrm{f}=0 \mathrm{~s}^{-1}$ & 42.4 & $\infty$ & $\infty$ & $\infty$ & 40.1 \\
$\mathrm{f}=10^{-5} \mathrm{~s}^{-1}$ & 42.3 & 9.0 & 381.6 & 728.2 & 40.0 \\
$\mathrm{f}=5 \times 10^{-5} \mathrm{~s}^{-1}$ & 40.2 & 1.61 & 76.3 & 5.83 & 37.5 \\
$\mathrm{f}=10^{-4} \mathrm{~s}^{-1}$ & 33.4 & 0.56 & 38.2 & 0.73 & 32.7 \\
\hline
\end{tabular}

Table 1: Velocity and $\tan \beta$ for the experiments in Fig. 1 for the two different methods of solving the streamtube equations.

\begin{tabular}{|c|c|c|c|c|c|}
\hline & \multicolumn{2}{|c|}{ Method 1 } & \multicolumn{2}{c|}{ Method 2 } & Observed \\
\cline { 2 - 6 } & $\mathrm{V}_{1}\left(\mathrm{~cm} \mathrm{~s}^{-1}\right)$ & $\tan \beta_{1}$ & $\mathrm{~V}_{2}\left(\mathrm{~cm} \mathrm{~s}^{-1}\right)$ & $\tan \beta_{2}$ & $\mathrm{~V}\left(\mathrm{~cm} \mathrm{~s}^{-1}\right)$ \\
\hline $\mathrm{C}_{d}=0.001$ & 38.1 & 0.07 & 38.2 & 0.07 & 43.1 \\
$\mathrm{C}_{d}=0.005$ & 36.3 & 0.32 & 38.2 & 0.36 & 37.5 \\
$\mathrm{C}_{d}=0.01$ & 33.4 & 0.56 & 38.2 & 0.73 & 32.7 \\
$\mathrm{C}_{d}=0.05$ & 23.0 & 1.32 & 38.2 & 3.64 & 19.1 \\
\hline
\end{tabular}

Table 2: Velocity and $\tan \beta$ for the experiments in Fig. 2 for the two different methods of solving the streamtube equations.

In all cases with rotation, the path predicted by Method 1 agrees well with the modeled plume. For $f=5 \times 10^{-5} \mathrm{~s}^{-1}$, for example, the angle predicted by Method 1 is close to the angle of center of the plume, and the predicted velocity, $40.2 \mathrm{~cm} \mathrm{~s}^{-1}$, is close to the measured value of $37.5 \mathrm{~cm} \mathrm{~s}^{-1}$. Similar agreement is found in all cases with rotation.

In contrast with the predictions by Method 1, the agreement between the path predicted with Method 2 and the modeled plume depends on the rotation rate. The path predicted by Method 2 agrees well at the highest rotation rate, $f=10^{-4} \mathrm{~s}^{-1}$, but diverges from the model at lower rotation rates. This discrepancy exists because the flow is not in geostrophic balance. Method 2 assumes that the flow is in geostrophic balance; consequently, considering the Rossby number of the flow will give an indication of the appropriateness of Method 2. Using the same scalings as used in section 2 (length $\approx 40 \mathrm{~km}$, velocity $\approx 40 \mathrm{~cm} \mathrm{~s}^{-1}$ ), for $f=10^{-5} \mathrm{~s}^{-1}, f=5 \times 10^{-5} \mathrm{~s}^{-1}$, and $f=10^{-4} \mathrm{~s}^{-1}$, the Rossby number is 5,1 , and 0.1 , respectively. These values of the Rossby number indicate that Method 2 is not appropriate for $f=10^{-5} \mathrm{~s}^{-1}$ and $f=5 \times 10^{-5} \mathrm{~s}^{-1}$. This is also apparent in a comparison between the geostrophic velocity and the observed velocity. For the case of $f=5 \times 10^{-5} \mathrm{~s}^{-1}$, for example, the measured velocity of the plume is $37.5 \mathrm{~cm} \mathrm{~s}^{-1}$, while the geostrophic velocity is $76.3 \mathrm{~cm} \mathrm{~s}^{-1}$.

We also examined the impact of bottom friction on the outflow's path. Figure 2 shows the height of the intruding current and the path predicted by the streamtube model for the two different solution methods, on day five of the integration, for four different values of bottom friction. The experimental setup is identical to that used to produce Fig. 1, except that in this case, the rotation rate is held constant at $10^{-4} \mathrm{~s}^{-1}$ and the bottom friction coefficient is varied. The panels in Fig. 2 show the outflow path that results with bottom friction values of 0.001 (upper left panel), 0.005 (upper right panel), 0.01 (lower left panel), and 0.05 (lower right panel). For comparison, typical values of bottom friction used in ocean 

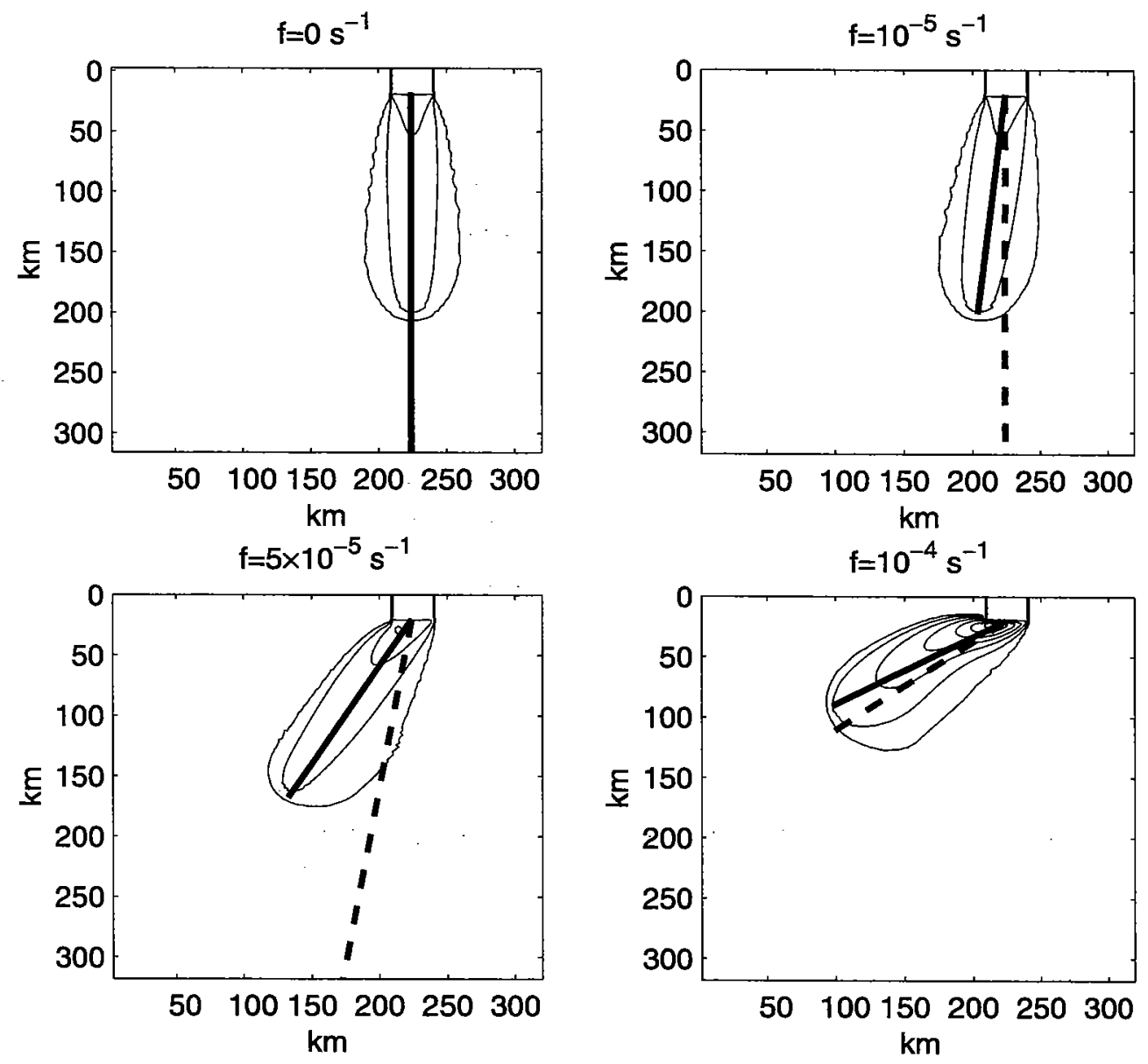

Figure 1: Plume height (thin contours) and path predicted by streamtube theory with Method 1 (thick solid line) and Method 2 (thick dashed line), on day 5 for different rotation rates: $0.0 \mathrm{~s}^{-1}, 10^{-5} \mathrm{~s}^{-1}$ (top right), $5 \times 10^{-5} \mathrm{~s}^{-1}$ (bottom left), $10^{-4} \mathrm{~s}^{-1}$ (bottom right) for a slope of 0.008 and a bottom drag $\left(\mathrm{C}_{d}\right)$ of 0.01 . Contour interval is $10 \mathrm{~m}$. 

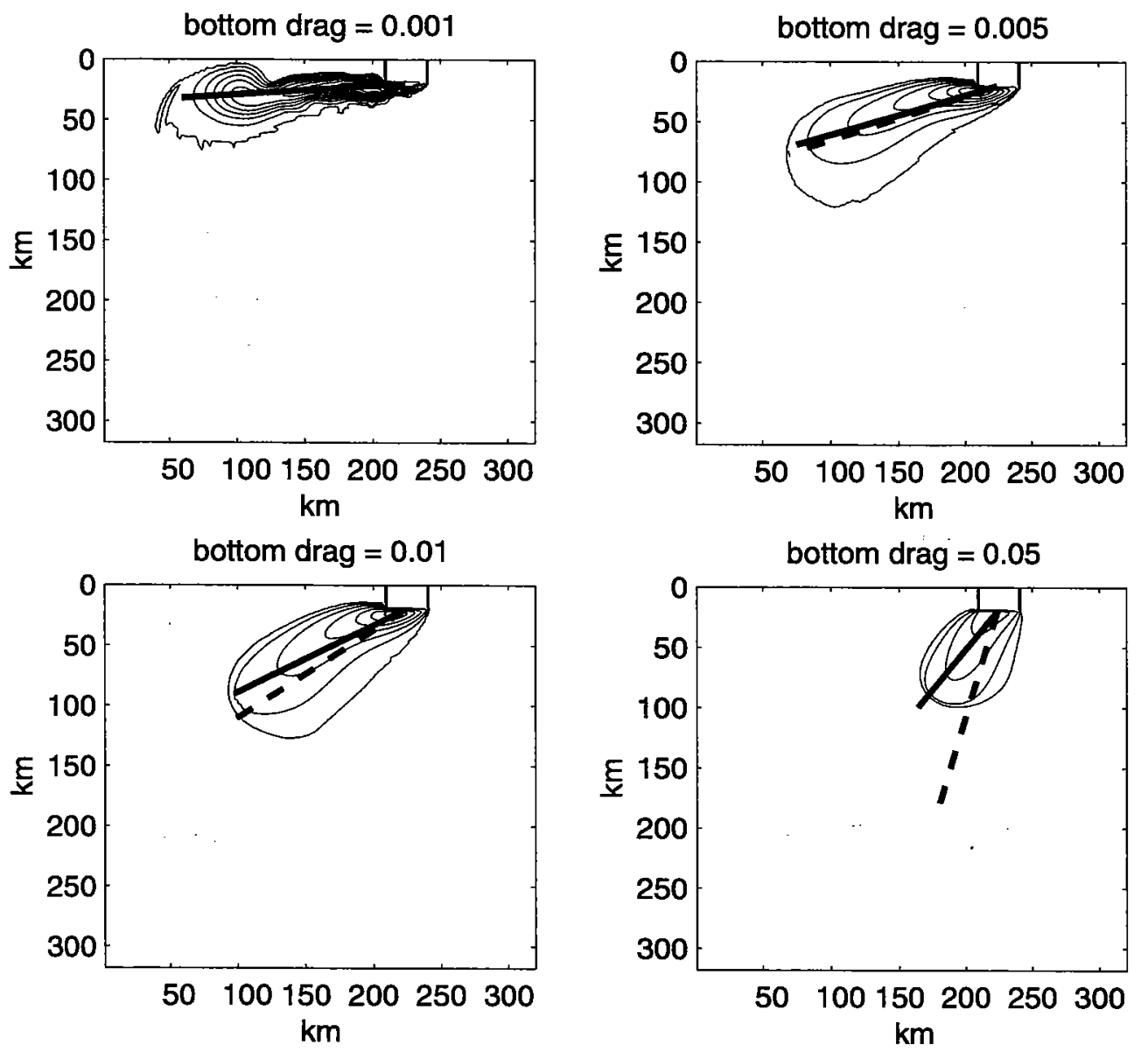

Figure 2: Plume height (thin contours) and path predicted by streamtube theory with Method 1 (thick solid line) and Method 2 (thick dashed line), on day 5 for different values of bottom drag $\left(\mathrm{C}_{d}\right): 0.001$ (top left), 0.005 (top right), 0.01 (bottom left), 0.05 (bottom right) for a slope of 0.008 and a rotation rate of $10^{-4} \mathrm{~s}^{-1}$. Contour interval is $10 \mathrm{~m}$. 
modeling are around 0.003 . It is clear from Fig. 2 that as bottom friction is increased, the gravity plume is able to cross lines of constant depth with an increasingly steep angle. For the smallest value of bottom friction, 0.001 , the flow is almost parallel to topography. This is consistent with the simplest (i.e. inviscid) models of outflows that predict alongslope flow.

In all cases, there is reasonably good agreement between the path of the model and the path predicted by the two methods of solving the streamtube equations. For lower values of bottom friction, $\mathrm{C}_{d}=0.001$ and $\mathrm{C}_{d}=0.005$, the two methods give very similar predictions. This is seen in Table 2, which shows the velocity and Ekman number predicted by the two solution methods and the observed velocity of the plume for the cases shown in Fig. 2. For example, the velocities predicted by Method 1 and Method 2 for the case when bottom friction is 0.005 are $36.3 \mathrm{~cm} \mathrm{~s}^{-1}$ and $37.5 \mathrm{~cm} \mathrm{~s}^{-1}$, respectively.

At higher values of bottom friction $\left(\mathrm{C}_{d}=0.01\right.$ and $\left.\mathrm{C}_{d}=0.05\right)$, the path predicted by Method 2 is more downhill than the path predicted by Method 1. This is due to the decelerating influence of friction, which prevents the plume from achieving geostrophic balance. At higher values of friction, the measured velocity is substantially less than the geostrophic velocity. For example, when bottom friction is 0.05 , the measured velocity of the plume is approximately $19.0 \mathrm{~cm} \mathrm{~s}^{-1}$, while the geostrophic velocity is $38.2 \mathrm{~cm} \mathrm{~s}^{-1}$. The velocity predicted by Method 1 adjusts to the higher value of friction, resulting in a more accurate prediction of the plume's path.

One can also apply the streamtube model in a local sense by considering each point in the domain as an individual streamtube with its own mass flux. In this case, the velocity and height of the flow are diagnosed at each grid point, and these fields are substituted into equation (7). The resulting field gives information about the path that is preferred locally, in contrast with the bulk streamtube which gives the preferred path of the bulk plume. Figure 3 shows the 'local streamtube' field (arrows) ${ }^{4}$ for the case when bottom friction is 0.005 (the same case shown in the upper right panel of Fig. 2). Also shown in Fig. 3 are the predictions by the two different methods of solving the streamtube equations. Near the center of the plume, the local streamtube field is oriented in a direction nearly parallel to the bulk streamtube approximation. Away from the center of the plume, however, the height of the plume tends to decrease, resulting in a corresponding decrease in the local mass flux. The decrease in the local mass flux causes the local streamtube field to diverge from the direction predicted for the bulk plume. Price and Baringer[2] suggest that bottom friction might play a role in the spreading of outflows. Figure 3 suggests a mechanism through which this spreading might occur: as fluid moves away from the center of the plume, the local Ekman number of the flow rises, indicating an increase in the relative importance of friction. The increase facilitates the downhill motion of the fluid, leading to a spreading of the outflow.

\footnotetext{
${ }^{4}$ Note that the field plotted in Fig. 3 is normalized so that the length of each arrow is 1 ; the length of the arrows does not indicate the magnitude of the local Ekman number, only the direction that the local Ekman number predicts for the flow
} 


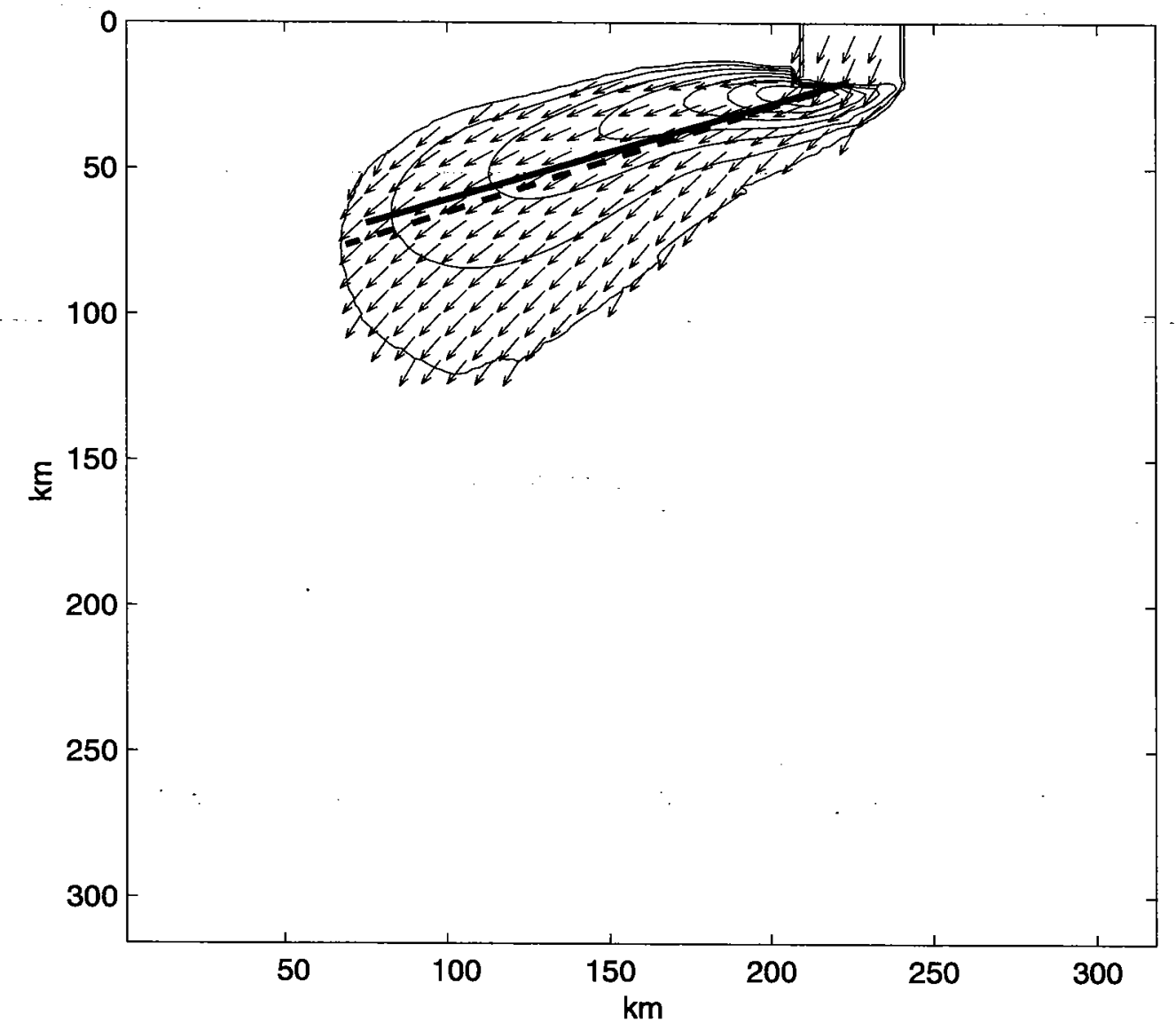

Figure 3: Thickness (thin contours-interval $10 \mathrm{~m}$ ), bulk streamtube model (Method 1thick solid line; Method 2-thick dashed line), and local streamtube (arrows), on day 5 of integration for a bottom drag value of 0.005 


\section{Turbidity current}

\subsection{Nof's (1996) turbidity current theory}

Nof proposes a simple analytic model to explain the path of the turbidity current associated with the 1929 Grand Banks earthquake. Rather than consider the complex processes that lead to the formation of the turbidity current, Nof simplifies the problem by treating the turbidity current as a geostrophically adjusted "blob" of fluid on parabolic slope. Nof makes further simplifications by treating the shape of the blob as constant in time and by neglecting the effect of viscosity and bottom drag. For brevity, we only present the solution; for the derivation of Nof's model, see Nof (1996).

With the simplifications discussed above, the motion of the blob of fluid is given by the following two components:

$$
\begin{gathered}
X(t)=\frac{-g^{\prime} T_{1} f}{\left(f^{2}+2 g^{\prime} T_{2}\right)^{\frac{3}{2}}}\left(\left[f^{2}+2 g^{\prime} T_{2}\right]^{\frac{1}{2}} t-\sin \left[\left(f^{2}+2 g^{\prime} T_{2}\right)^{\frac{1}{2}} t\right]\right) \\
Y(t)=\frac{-g^{\prime} T_{1}}{\left(f^{2}+2 g^{\prime} T_{2}\right)}\left(1-\cos \left[\left(f^{2}+2 g^{\prime} T_{2}\right)^{\frac{1}{2}} t\right]\right)
\end{gathered}
$$

where $X$ and $Y$ are position of the center of the blob as a function of time, $t, g^{\prime}=g \frac{\Delta \rho}{\rho}$ is the reduced gravity, $f$ is the Coriolis parameter, and the constants $T_{1}$ and $T_{2}$ describe the parabolic topography, such that the topographical height can be written as $z=T_{1} y+T_{2} y^{2}$ (in all cases considered below, $T_{2}=0$ ).

\subsection{Comparison between Nof's theory and model results}

To test Nof's theory of the 1929 Grand Banks earthquake and to investigate flow with a relatively high Rossby number, we integrated the model in the turbidity current configuration described in section 2. We considered the evolution of a Gaussian "blob" of fluid with a radius of approximately $100 \mathrm{~km}$ and a maximum height of $150 \mathrm{~m}$ on linear topography with a slope of 0.006 (similar values to those considered in Nof) over a two day period. Figure 4 shows the height of the blob every 6 hours (thin contours) over the first day of integration and the path predicted by Nof's theory (thick line) for the center of the blob. Figure 4 also shows, as a heavy red contour (stippled in black and white), the regions where the Rossby number ${ }^{5}$ is larger than 1 . The beginning Nof's theory (around $X=600 \mathrm{~km}, Y=180 \mathrm{~km}$ ) marks the center of the blob at $t=0$. For the first 6 hours (upper left panel in Fig. 4), the blob roughly follows the path predicted by Nof's theory, deflecting to the blob's right under the influence of the Coriolis force. After the first 6 hours, however, the blob's path diverges substantially from Nof's theory. Note that in contrast with Nof's theory, which assumes that the blob maintains its shape, the blob has deformed substantially during this interval.

At 12 hours (upper right panel in Fig. 4), the fluid that was initially in the blob begins to collect in a region (around $X=400 \mathrm{~km}, Y=550 \mathrm{~km}$ ) that is characterized by high velocities $\left(\sim 6-10 \mathrm{~m} \mathrm{~s}^{-1}\right)$. This rapidly moving region, which we call "the drip" region, grows throughout the rest of the simulation, such that by the end of the first day (lower

\footnotetext{
${ }^{5}$ To calculate the Rossby number, we define the length scale as $75 \mathrm{~km}$, approximately the radius of the initial Gaussian plume
} 
right panel in Fig. 4), most of the fluid that was present in the initial Gaussian blob is in the drip region. The velocities in the drip region are large enough that the nonlinear terms in equation (1) become important. The importance of the nonlinear terms can be seen by looking at the local Rossby number. At 6 hours into the simulation (upper left panel), the Rossby number is larger than 1 (maximum value of approximately 1.6) towards the bottom right-hand side (towards $\mathrm{x}=0, \mathrm{y}=1000$ ) of the blob. As the simulation proceeds, the drip region forms in the area where the Rossby number is greater than 1 , indicating the importance of non-linear terms in the formation of the drip region.

Figure 5 shows the height of the blob (thin contours) and the path predicted by Nof's theory (thick line) for a case that is identical to the case shown in Fig. 4, but with topographical slope of 0.003 , half of the value used in the simulation shown in Fig. 4. In this case, a drip region does not form. In addition, the blob of heavy fluid follows Nof's theory for a longer time; the blob's path agrees reasonably well with the theory for approximately 12 hours. In this case, the Rossby number of the flow (not visible), calculated in the same manner as in Fig. 4, never exceeds 1. Instead, the Rossby number of the flow has a maximum value of approximately 0.75 . Is is interesting to note that if this same case is considered with a smaller value of bottom friction, shown in Fig. 6, the Rossby number of the flow does exceed 1, and, on day one, the flow begins to develop a drip region similar to that shown in Fig. 4.

\section{Conclusion}

In an effort to understand the processes that allow an oceanic gravity current to flow downhill, we have modeled gravity currents in two different flow regimes. First, we considered the motion of outflows, which in the midlatitudes are approximately in geostrophic balance but are modified by the presence of friction. The simplest available model-the bulk streamtube model-predicts that the presence of friction will allow the outflow to propagate downhill. Our dynamical model of an outflow is consistent with the simple streamtube model: as we increase bottom friction, the motion of the outflow is increasingly downhill. We also extended the streamtube model so that it can be applied in a local sense. Applying the streamtube model in this manner gives information about how an outflow plume spreads as it travels.

The second flow regime that we considered is the flow of a turbidity current. In this case, the velocities associated with the motion are high enough $\left(\sim 10 \mathrm{~m} \mathrm{~s}^{-1}\right)$ that the motion cannot be described by the geostrophic approximation. We compared the motion of a Gaussian blob of fluid, taken to represent a turbidity current after the initial formation stages, with the motion predicted by Nof's theory of turbidity currents. We found that for an initial period, around 6 hours, the motion of the blob was close to the Nof's theory. After this initial period, however, the blob of fluid diverged from the cycloid motion predicted by Nof. The difference between Nof's theory and the model is likely due to two factors. First, Nof's theory assumes that the blob does not change shape as it propagates. This is a severe restriction, imposed to simplify the mathematics. Our model does not impose any restrictions on the evolution of the blob's shape; indeed, as shown in the Fig. 4, the shape of the plume changes dramatically in time. Second, Nof's model assumes that there is no bottom friction and that the flow is inviscid. For reasons of numerical stability, we are not 

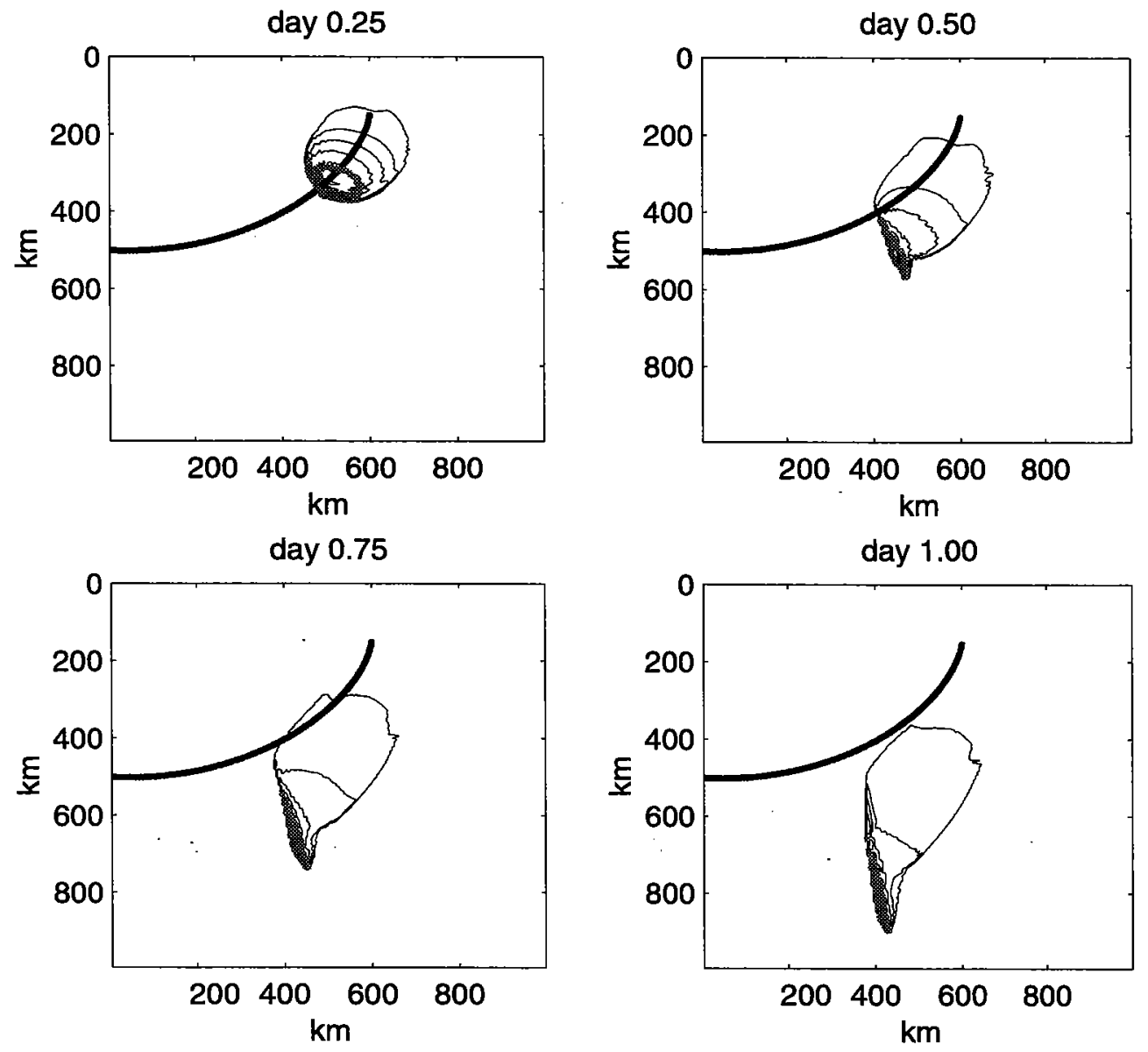

Figure 4: Thickness (contours) and Nof theory (heavy blue line) for day 0.25 (top left), day 0.5 (top right), day 0.75 (bottom left), and day 1.00 (bottom right) of turbidity current simulation with slope 0.006 and $f=10^{-4} \mathrm{~s}^{-1}$. Areas with Rossby number greater than 1 are shown with a heavy red contour (stippled in black and white). Height contour interval is $10 \mathrm{~m}$. 

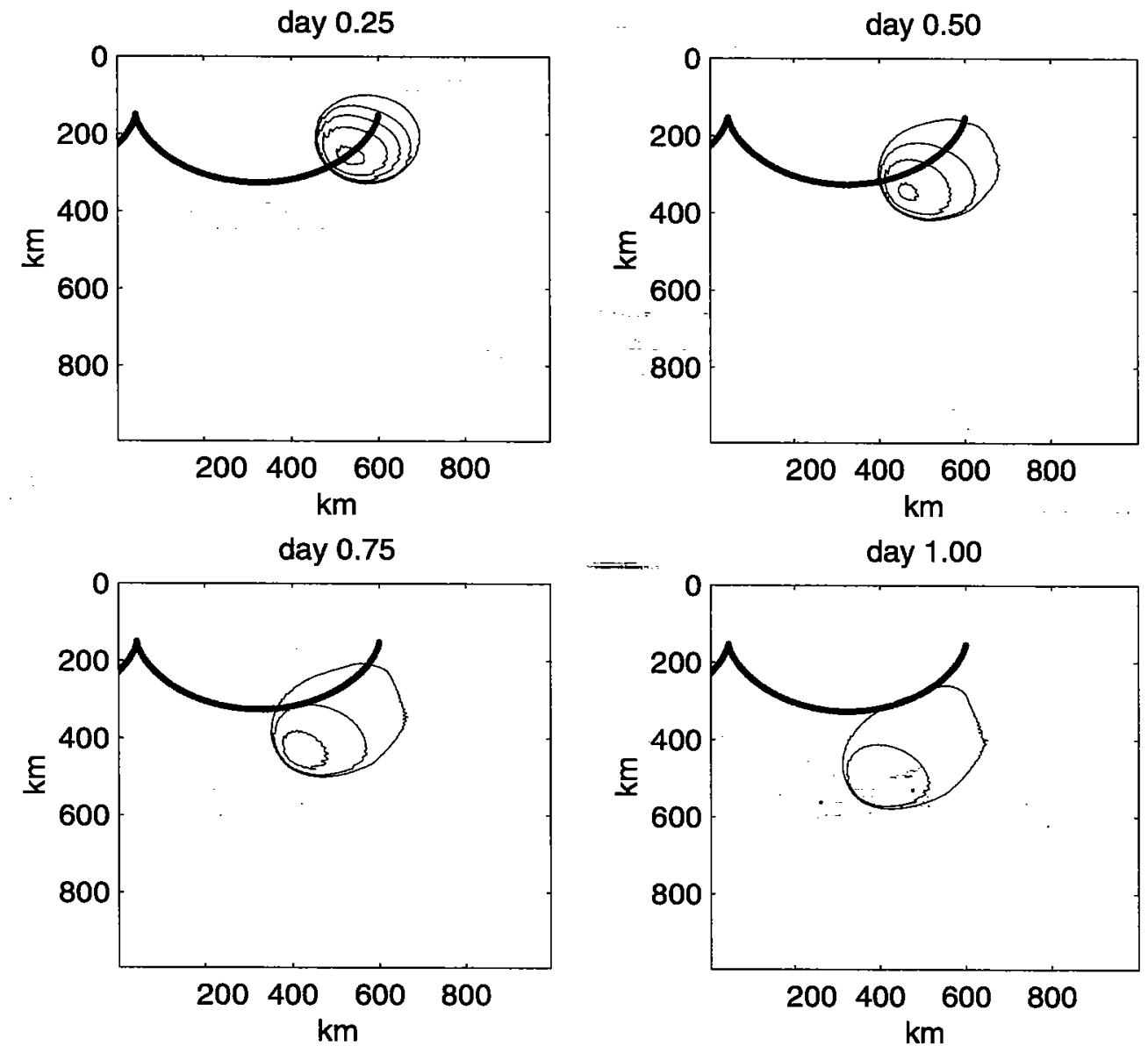

Figure 5: Thickness (contours) and Nof theory (heavy blue line) for day 0.25 (top left), day 0.5 (top right), day 0.75 (bottom left), and day 1.00 (bottom right) of turbidity current simulation with slope 0.003 and $f=10^{-4} \mathrm{~s}^{-1}$. Contour interval is $10 \mathrm{~m}$. 

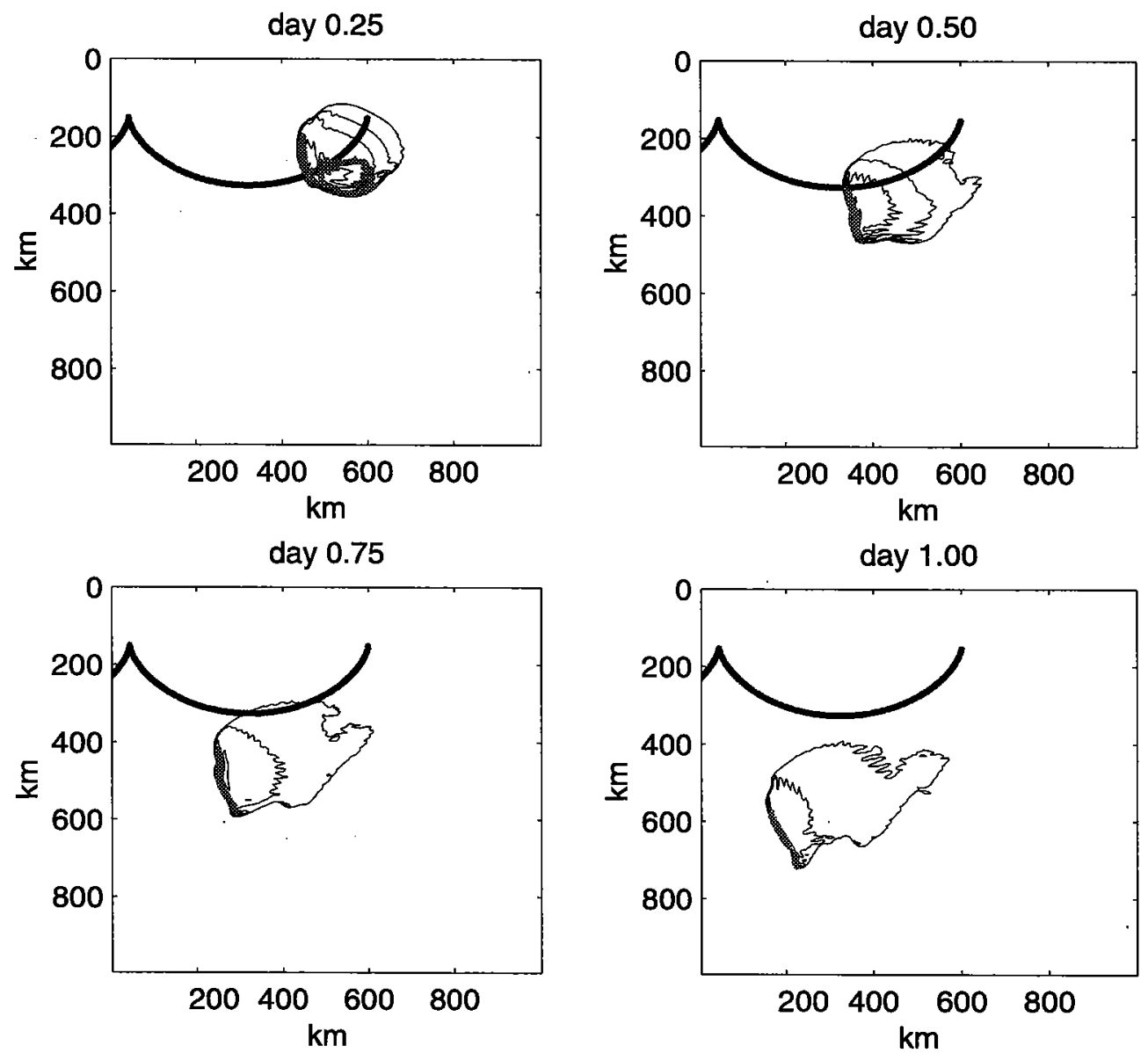

Figure 6: Thickness (contours) and Nof theory (heavy blue line) for day 0.25 (top left), day 0.5 (top right), day 0.75 (bottom left), and day 1.00 (bottom right). Areas with Rossby number greater than 1 are shown with a heavy red contour (stippled in black and white). Same case as Fig. 5, except with bottom friction of 0.001 . Contour interval is $10 \mathrm{~m}$. 
able to duplicate these assumptions, although we did investigate the effect of viscosity and bottom drag on the solution.

We also found that in some cases, the turbidity current collects into a small, rapidly moving area. The formation of this area is dependent on the Rossby number of the flow. Flows with a large $(>1)$ Rossby number develop this rapidly moving area, while flows with a smaller Rossby number do not. This feature is intriguing, and further study of its development is planned.

\subsection{Future work}

There are several issues related to the flow of turbidity currents that warrant further exploration. Specifically, several questions remain regarding the formation of the drip region:

1. What is the dependence on model resolution?

To date, we have only run the turbidity current simulations with a horizontal resolution of $5 \mathrm{~km}$. The small scales seen in the drip region indicate that a higher resolution may be necessary to fully resolve the details of the flow in this region.

2. Does the drip appear when other-more appropriate-models are used?

MICOM is intended to model the ocean circulation on the scale of an ocean basin. The extreme conditions (i.e. high velocities and steep height gradients) in the drip region may make the use of MICOM inappropriate for such a simulation. In the simulations shown in Fig. 4, the Froude number of the flow reached a minimum of about 1.8 in the drip region, indicating that the flow is close to a shock wave. Verifying the MICOM results with a shock resolving (i.e. energy and momentum conserving) model would lend credence to the results obtained using MICOM.

3. How does the upper layer influence the dynamics of the current?

Recent papers $[8,9]$ have shown that the ambient fluid can influence the dynamics of the intruding gravity current. We briefly investigated the role of the depth of the ambient fluid and found little influence on the dynamics of the gravity current. More investigation in this area-including the role of ambient stratification-is warranted.

4. How does entrainment/detrainment affect the dynamics of the turbidity current?

Entrainment and detrainment are major factors in the dynamics of gravity currents [5]. A full study of the dynamics of turbidity currents must include an investigation of the role of entrainment.

\section{Acknowledgments}

I am greatly indebted to Eric Chassignet for his guidance this summer. He was an excellent mentor in every way and I am grateful to him for the time that he spent with me. I also appreciate conversations with Jim Price throughout the summer. Besides the valuable comments he offered during our conversations, Jim referred me to the paper by Nof[10] which was instrumental in this project. Less directly related to this project, I would like 
to thank the staff and visitors of the 2000 GFD program, in particular the organizer, Rick Salmon, and the principal lecturer, Issac Held. Finally, thanks to the other GFD Fellows: Cheryl, Chris, Christos, Giulio, Karen, Lucy, Tivon, and Zhiming. It was a great group to spend a summer with. 


\section{References}

[1] J. E. Simpson, "Gravity currents in the laboratory, atmosphere, and ocean," Ann. Rev. Fluid Mech. 14, 213 (1982).

[2] J. F. Price and M. O. Baringer, "Outflows and deep water production by marginal seas," Deep-Sea Res. 33, 161 (1994).

[3] R. W. Griffiths, "Gravity currents in a rotating system," Ann. Rev. Fluid Mech. 18, 59 (1986).

[4] P. C. Smith, "A streamtube model for bottom boundary currents in the ocean," DeepSea Res. 22, 853 (1975).

[5] J. H. Jungclaus and J. O. Backhaus, "Application of a transient reduced gravity plume model to the Denmark Strait Overflow," J. Geophys. Res. 99, 12375 (1994).

[6] L. Jiang and R. W. Garwood, "A numerical study of three-dimensional dense bottom plumes on a slope," J. Geophys. Res. 100, 18471 (1995).

[7] L. Jiang and R. W. Garwood, "Three-dimensional simulations of overflows on continental slopes," J. Phys. Ocean. 26, 1214 (1996).

[8] G. F. Lane-Serff and P. G. Baines, "Eddy formation by dense flows on slopes in a rotating fluid," J. Fluid Mech. 363, 229 (1998).

[9] G. F. Lane-Serff and P. G. Baines, "Eddy formation by overflows in stratified water," J. Phys. Ocean. 30, 327 (2000).

[10] D. Nof, "Rotational turbidity flows and the 1929 Grand Banks earthquake," Deep-Sea Res. 43, 1143 (1996).

[11] J. R. Holton, An Introduction to Dynamic Meteorology (Academic Press, ADDRESS, 1992).

[12] D. B. Haidvogel and A. Beckmann, Numerical Ocean Circulation Modeling (Imperial College Press, ADDRESS, 1999).

[13] P. C. Smith, Ph.D. thesis, Massachusetts Institute of Technology, Cambridge, Mass., 1973. 


\title{
Experimental investigation of theory for stratified ocean convection
}

\author{
Chris Sonekan
}

\section{Introduction}

The ocean forms over seventy percent of the surface area of the earth. It is endowed with rich marine life of immense biological diversity and other interesting phenomena. Just as the atmosphere is inextricably linked to the oceans, so also man and his activities are connected with the oceans in a sort of symbiotic relationship. The teeming biological diversity and water have supplied some of the resources needed for the survival of man. On the other hand, the activities of man produce nutrients and minerals required by marine organisms lower on the food chain. Oceans also have a kind of thermostatic control on climate as they absorb and release water and carbon dioxide, as well as other gases.

However, not all characteristic features of the ocean are well understood. Between the warm well-mixed surface layer and the cold waters of the main body of the ocean is the thermocline, the zone within which temperature decreases markedly with depth [1]. The density of the oceans is dependent mainly on pressure, temperature, and salinity. The ocean has a unique density structure. The density field varies significantly in all three spatial directions, with the largest variations occurring in the upper two kilometers. This suggests stratification of density and other properties. A complete dynamical theory should explain and predict the three-dimensional variation of the density and velocity field. "This is the problem of the thermocline. It is non-linear and difficult" [2]. In addition to this, "the three-dimensional structure of the oceans is complex and very poorly understood" [1]. Moreover, Michael McIntyre [3] makes a similar comment, "ocean circulation - ill understood: a profoundly different and more difficult problem."

The vertical, density-driven circulation that results from cooling and/or increase in salinity, that is, changes in the heat and/or salt, is known as thermohaline circulation. In certain polar regions water, that has been subjected to extreme cooling, sinks and flows equatorward in the thermohaline circulation [1]. In a few localized regions, the circulation is confined to a small area and is pronounced in the vertical direction leading to the formation of convection cells.

Comprehensive studies have been done on ocean convection and even expeditions have been undertaken to carry out field experiments on the phenomenon. Killworth [4] gives a qualitative description of convection as it occurs in the World Ocean. Two types of convection are illustrated - continental boundary and open-ocean convection. The former occurs on continental shelf slope systems, as typified by various locations around the Antarctic coast. "The freezing of sea-ice, and resulting brine ejection, creates dense salty water on the shelf which descends the slope under a balance of Coriolis, gravity, and frictional forces, entraining the surrounding warm deep water as it goes." The second process occurs in locations such as the Mediterranean, the Labrador Sea, and two locations in the Weddell gyre, and is hypothesized to occur in the Greenland Sea. Openocean convection has many similarities in all these areas: it occurs in narrow (20-50 km) areas; it 
forms about $10^{3} \mathrm{~m}^{3} / \mathrm{s}$ of deep water; it occurs in regions of cyclonic mean circulation; more than one water mass in the mean circulation is involved; a preconditioning seems to be required; some surface forcing (cooling or sea-ice formation) is necessary; a violent breaking up of water mass frequently occurs on time scales of two weeks. Preconditioning refers to the weakening of vertical static stability in the surface layer. This results from a 'doming' of the isopycnal surfaces in the cyclonic gyre [4].

Field experiments undertaken to study oceanic convection include The Labrador Sea Deep Convection Experiment [5] and The Medoc Project [6]. Rudels [7] reports of convection observed in the Greenland Sea in the winter of 1987-1988. A study or summary of theoretical models of the thermohaline circulation has been done by Veronis [8]. And Whitehead [9] presents a summary of the models of the thermohaline ocean processes.

A new laboratory experiment has been undertaken that follows from the analytical model developed by Whitehead (manuscript in preparation). He showed that two states of flow for the same localized surface cooling conditions might exist for a salt-stratified isothermal body of water. In one case, freshwater is cooled and convective circulation is shallow. In the second state, salty water is entrained and deep convection of mixed water occurs. The two flows can be found for the same driving parameters in a certain range. Some special conditions are required for the multiple equilibrium flow to exist in this configuration. For example, it is necessary for the resistance of flow for the surface water to be greater that the resistance of deep flow. A numerical analysis of the model was done. The purpose of this project was to design, construct, and test a laboratory apparatus that might demonstrate the two flow-states.

\section{Method}

A simple physical model for investigating the theory for convection was built in the laboratory. The apparatus consists of two chambers - a small chamber (a vertical cylinder $5 \mathrm{~cm}$ in diameter and $23.9 \mathrm{~cm}$ deep), and a bigger one (a rectangular box measuring $21.8 \mathrm{~cm}$ in length, $22 \mathrm{~cm}$ in width, and 21.5 deep). Both were connected laterally with three tubes of different diameters close to the top, the middle, and close to the bottom. Tube spacing was $9.5 \mathrm{~cm}$. Tube diameters were $0.9,0.6$, and $0.3 \mathrm{~cm}$, respectively. The small chamber had a copper base. The copper bottom is encased in a metal cup connected to two plastic tubes on both sides. The other ends of the tubes are connected to a hot water bath. The bigger vessel also contains three other hoses - one brings in saltwater to the bottom, the other brings in freshwater to the top, and the third hose removes a mixture of both waters at the interface of the two fluids. The two in flows were pumped at a steady rate. Both containers were filled with a layer of salt water about $3.5 \mathrm{~cm}$ thick, on top of which is a layer of freshwater about five times as deep. The bigger chamber is kept at about $20^{\circ} \mathrm{C}$. A marker - blue dye - identified the layer of saltwater, while the freshwater is unmarked and stays colorless. The hot-water-bath temperature range for the experimental runs was between 24.3 and $30^{\circ} \mathrm{C}$. The water bath's systems automatically recorded the temperature of the bath in digital mode, while the temperature of the top, mid-depth, and bottom of both chambers were measured with a standard thermometer. By depositing a red color dye at the mouth of the connecting tubes, the flow direction and time were measured. Samples of fluid were extracted from the top, middle, and bottom portions of the fluid, and the density was measured with densiometer. A Matlab program was used to find the equivalent salinity of the density. Earlier runs were made at halfhourly and hourly intervals, and later runs were made at half-hourly intervals.

In his analytical and numerical model in progress, Whitehead (2000) assumed flow through the tubes are governed by laminar viscous flow laws, so that flux through each tube is proportional to the pressure difference across the tube. Effects of rotation were neglected. 


\section{Data}

The measured quantities are accumulated in seven tables, which are too long and detailed for this report. The temperature of the fluid at the top, the middle, and bottom portions of the fluid are tabulated for different bath temperatures. Also, the time of flow, density, and salinity are presented. In addition to these, the dimensions of the chambers are given.

\section{Results}

Experimental results for the flow rates through the top, middle, and bottom connecting tubes are summarized in Table 10 (not shown). Theoretical calculations for the flow rates are also presented in two tables. According to theory, for limited cooling, only the top and middle tubes are involved in vigorous circulating fluid as happens in a convection cell. A comparison of results for theory and experiment show disparity of considerable proportions. Theory predicts that for strong cooling, all three connecting tubes circulate fluid. Again, comparison of theoretical calculations of flow rates and values obtained from experiment indicate poor agreement between theory and experiment.

\section{Discussion}

The dynamics as determined by theory suggests that several stages of flow exist for small, moderate, strong, and very strong cooling. Agreement between experiment and theory was very poor, and the reason for this is unknown at this time. Possibly, the resistance coefficient of the bottom tube was too great. New experiments with a smaller resistance coefficient for the bottom tube are continuing with much better agreement between experiment and theory (Whitehead, Private Communication). In this experiment a major change from theory was made regarding the effect of heat on the fluid system. The theory focuses on heat removal from the variant chamber and ignores the effect of conduction of heat in the body of the fluid. Also, while cooling makes the fluid column dynamically unstable from the top, heating triggers rising motion from the bottom. Moreover, the gradient of temperature or pressure on both sides of the connecting tube may be different for the case of cooling.

Experimental errors may have been incurred as measurements were made manually and recorded. The heating bath temperature was unsteady most of the time, and the device would not adjust to a temperature of about $24^{\circ} \mathrm{C}$.

Certain approximations were made in the computations. Since the saltwater was one part ocean water and nine parts freshwater, the salinity was different from pure ocean water. Using the appropriate equation-of- state parameters, such as specific values of thermal expansion coefficient and salt contraction coefficient, could have improved the theoretical values and made them more realistic. The approximations used may underestimate the effect of low salinity levels on the density, and, hence, the effects on the values of the flow rate. Eventually the integrity of the dynamics of the system may be undermined by such estimates, causing major differences between the results of theory and experiment.

\section{Conclusion}

An attempt has been made to check the authenticity of a theory for oceanic convection. To do this, a laboratory experiment was done. The laboratory method differed from the theory. Heating from underneath the fluid chamber instead of cooling from above, may have altered the dynamics and caused theory and experiment results to agree less. In spite of this drawback, the experiment 
went well. Ample evidence of convection was demonstrated. The theory, being under development, may probably work for a system that is cooled from the top. Possibly the experimental data may provide grounds for further development of the theory.

\section{Acknowledgements}

A special thanks is credited to Dr. Jack Whitehead who was a source of encouragement and inspiration to me.

\section{References}

[1] Brown et al., "Ocean circulation," Open University Oceanography Course Team. Pergamon Press, New York (1996).

[2] J. Pedlosky, “Ocean circulation theory,” Springer-Verlag, Germany (1996).

[3] M. McIntyre, "On global scale atmospheric circulations and remarks on oceans and solar spinoff," Geophysical Fluid Dynamics, Summer 2000, Talk/Seminar (2000).

[4] P. D. Killworth, "Deep convection in the world oceans," Reviews of Geophysics and Space Physics 21, 1-26 (1983).

[5] Lab Sea Group, "The Labrador sea deep convection experiment," Bulletin of the American Meteorological Society 10, 2033-2058 (1998).

[6] Medoc Group, "Observations of formation of deep water in the Mediterranean Sea.," Nature, 227, 1037-1040 (1969).

[7] B. Rudels, "Greenland Sea convection in winter of 1987-1988," Journal of Geophysical Research 94 (C3), 3223-3227 (1989).

[8] G. Veronis, "On Theoretical models of the thermocline circulation," Deep Sea Research 16, 301-323 (1969).

[9] J. A. Whitehead. "Thermohaline Ocean Processes and Models," Annual Review of Fluid Mechanics 27, 89-114 (1995).

[10] J. A. Whitehead, "Stratified Convection with Multiple States," Ocean Modelling, In Press (2001). 


\begin{tabular}{|c|c|c|}
\hline $\begin{array}{l}\text { REPORT DOCUMENTATION } \\
\text { PAGE }\end{array}$ & $\begin{array}{l}\text { 1. REPORT NO. } \\
\text { WHOI-2001-03 }\end{array}$ & 3. Reciplent's Accession No. \\
\hline \multirow{2}{*}{\multicolumn{2}{|c|}{$\begin{array}{l}\text { 4. Title and Subtitle } \\
\text { The General Circulation of the Atmosphere: } 2000 \text { Program in Geophysical } \\
\text { Fluid Dynamics }\end{array}$}} & $\begin{array}{l}\text { 5. Report Date } \\
\text { March } 2001\end{array}$ \\
\hline & & 6. \\
\hline \multicolumn{2}{|c|}{ 7. Author(s) Richard L. Salmon, Director } & $\begin{array}{l}\text { 8. Performing Organization Rept. No. } \\
\text { WHOI-2001-03 }\end{array}$ \\
\hline \multirow{2}{*}{\multicolumn{2}{|c|}{$\begin{array}{l}\text { 9. Performing Organization Name and Address } \\
\text { Woods Hole Oceanographic Institution } \\
\text { Woods Hole, Massachusetts } 02543\end{array}$}} & 10. Project/Task/Work Unit No. \\
\hline & & $\begin{array}{l}\text { 11. Contract(C) or Grant(G) No. } \\
\text { (C) N00014-97-1-0934 } \\
\text { (G) OCE-9810647 }\end{array}$ \\
\hline \multirow{2}{*}{\multicolumn{2}{|c|}{$\begin{array}{l}\text { 12. Sponsoring Organization Name and Address } \\
\text { Office of Naval Research } \\
\text { National Science Foundation }\end{array}$}} & $\begin{array}{l}\text { 13. Type of Report \& Period Covered } \\
\text { Technical Report }\end{array}$ \\
\hline & & 14. \\
\hline
\end{tabular}

\section{Supplementary Notes}

This report should be cited as: Woods Hole Oceanog. Inst. Tech. Rept., WHOI-2001-03.

\section{Abstract (Limit: 200 words)}

The 2000 GFD program focused on "The General Circulation of the Atmosphere." Principal lecturer Isaac Held delivered the two-week introductory course, supported by the lectures of Alan Plumb, Wayne Schubert, Michael Cullen, and Oliver Bühler. The concept of pseudomomentum emerged as the underlying theme. This concept has virtually transformed meteorology within the past 20 years, yet it remains mostly unfamiliar to oceanographers and others. Thus the 2000 session perfectly fit the GFD mission of diffusing ideas across disciplinary boundaries. As the summer wore on, the range of topics broadened in its customary way to include: the general circulation of Jupiter and Mars; lattice-Boltzmann and gas-kinetic methods for solving the equations of fluid mechanics; the scattering of sound waves by vortices; the heating of the universe; and many more topics too numerous to list. For an accurate impression of the program's activities, the reader should be sure to scan the 2000 lecture schedule.

\section{Document Analysis a. Descriptors}

dynamics

atmosphere

circulation

b. Identifiers/Open-Ended Terms

c. COSATI Field/Group

18. Availability Statement

Approved for public release; distribution unlimited.

\begin{tabular}{|l|l|}
\hline $\begin{array}{c}\text { 19. Security Class (This Report) } \\
\text { UNCLASSIFIED }\end{array}$ & $\begin{array}{c}\text { 21. No. of Pages } \\
196\end{array}$ \\
\hline 20. Security Class (This Page) & 22. Price \\
\hline
\end{tabular}




\section{DOCUMENT LIBRARY}

\section{Distribution List for Technical Report Exchange - July 1998}

University of California, San Diego

SIO Library 0175C

9500 Gilman Drive

La Jolla, CA 92093-0175

Hancock Library of Biology \& Oceanography

Alan Hancock Laboratory

University of Southern California

University Park

Los Angeles, CA 90089-0371

Gifts \& Exchanges

Library

Bedford Institute of Oceanography

P.O. Box 1006

Dartmouth, NS, B2Y 4A2, CANADA

NOAA/EDIS Miami Library Center

4301 Rickenbacker Causeway

Miami, FL 33149

Research Library

U.S. Army Corps of Engineers

Waterways Experiment Station

3909 Halls Ferry Road

Vicksburg, MS 39180-6199

Marine Resources Information Center

Building E38-320

MIT

Cambridge, MA 02139

Library

Lamont-Doherty Geological Observatory

Columbia University

Palisades, NY 10964

Library

Serials Department

Oregon State University

Corvallis, OR 97331

Pell Marine Science Library

University of Rhode Island

Narragansett Bay Campus

Narragansett, RI 02882

Working Collection

Texas A\&M University

Dept. of Oceanography

College Station, TX 77843
Fisheries-Oceanography Library

151 Oceanography Teaching Bldg.

University of Washington

Seattle, WA 98195

Library

R.S.M.A.S.

University of Miami

4600 Rickenbacker Causeway

Miami, FL 33149

Maury Oceanographic Library

Naval Oceanographic Office

Building 1003 South

1002 Balch Blvd.

Stennis Space Center, MS, 39522-5001

Library

Institute of Ocean Sciences

P.O. Box 6000

Sidney, B.C. V8L 4B2

CANADA

National Oceanographic Library

Southampton Oceanography Centre

European Way

Southampton SO14 3ZH

UK

The Librarian

CSIRO Marine Laboratories

G.P.O. Box 1538

Hobart, Tasmania

AUSTRALIA 7001

Library

Proudman Oceanographic Laboratory

Bidston Observatory

Birkenhead

Merseyside L43 7 RA

UNITED KINGDOM

IFREMER

Centre de Brest

Service Documentation - Publications BP 7029280 PLOUZANE

FRANCE 